\title{
Fullerene-Nitroxide Derivatives as Potential Polarizers for Dynamic Nuclear Polarization (DNP) in Liquid State
}

\author{
Dissertation \\ for the award of the degree \\ "Doctor rerum naturalium" \\ Division of Mathematics and Natural Science \\ of the \\ Georg-August-University Göttingen
}

submitted by

Nikolay Enkin

from Moscow

Göttingen, 2015 


\section{Thesis committee}

Prof. Dr. Marina Bennati

Electron Spin Resonance Spectroscopy Group, Max Planck Institute for Biophysical Chemistry;

Institute for Organic and Biomolecular Chemistry, Georg August University, Göttingen

Prof. Dr. Markus Zweckstetter

Structure Determination of Proteins Using NMR Group, Max Planck Institute for Biophysical Chemistry

Prof. Dr. Jörg Enderlein

III. Institute of Physics, Georg August University, Göttingen

\section{Members of the Examination Board}

Referee: Prof. Dr. Marina Bennati

Electron Spin Resonance Spectroscopy Group, Max Planck Institute for Biophysical Chemistry

$\&$

Institute for Organic and Biomolecular Chemistry, Georg August University, Göttingen

$2^{\text {nd }}$ Referee: Prof. Dr. Markus Zweckstetter

Structure Determination of Proteins Using NMR Group, Max Planck Institute for Biophysical Chemistry, Göttingen

Prof. Dr. Jörg Enderlein

III. Institute of Physics, Georg August University, Göttingen

Dr. Gopalakrishnan Balasubramanian

Spin Imaging Group, Max Planck Institute for Biophysical Chemistry, Göttingen 


\section{Declaration}

I hereby declare that this thesis has been written independently and with no other sources and aids than quoted.

Signed:

Date: 31.07.2015

Nikolay Enkin, Dipl. Phys.

Göttingen 


\section{Acknowledgements}

I would like to express my sincere gratitude to my supervisor Professor Marina Bennati for giving me the chance to receive the position for a research work in her progressive modern scientific lab. I thank Marina for her patience and immense knowledge and very useful and sage advice.

I would like to thank my second advisor Dr. Igor Tkach very much for the continuous support to my Ph.D study, help to solve any instrumentation problems, explanation how everything works and how to avoid irreversible breakage of devices.

Besides my advisors, I would like to thank the rest of my thesis committee: Professor Jörg Enderlein and Professor Markus Zweckstetter for their insightful comments and reminding me about very important details which I often missed and neglected. I thank them for having time to discuss various questions about career opportunity.

I express my gratitude to Dr. Guoquan Liu for his invaluable contribution to my experimental work, sharing his skills and knowledge with me, help with synthesis of investigated molecules.

My sincere thanks also goes to Dr. Vladimir Belov and staff of facility for synthetic chemistry for their unrestricted help with development and synthesis of the molecules potentially interesting for our investigations.

I thank Dr. Müge Kasanmascheff, Roberto Rizzato, Dr. Thomas U. Nick, Isabel Bejenke, Karin Halbmair for regular helps and discussions which have extended my knowledge and improved my skills as a scientific researcher. Of course, the period of my stay in Göttingen would not be so much lively and multifaceted without my colleagues. Thanks to our former lab colleague Dr. Alistair Fielding, as native English speaker, for help with English language skills. To Monika Frömel and Brigitta Angerstein I am grateful for help to avoid wasting time for bureaucracy.

I also would like to thank our former workers: Dr. Giuseppe Sicoli, Dr. Tomislav Argirević, Dr. Maria-Teresa Hussels (Türke), Dr. Roberta Pievo, Dr. Soraya Pornsuwan for their welcome.

Finally, I thank staff of Precision Mechanics Workshop and IT \& Electronics Service for their help whenever I needed. 


\section{Abstract}

Nuclear magnetic resonance spectroscopy and imaging (NMR/MRI) are widely applied methods for non-destructive analysis of biological systems, materials and medical applications. Dynamic nuclear polarization (DNP) is a method to enhance NMR signal by means of polarization transfer from unpaired electrons to nuclei through saturation the electron spin transition by microwave (MW). DNP in liquids is governed by Overhauser mechanism which results from the simultaneous flipping of electron and nuclear spins. The DNP efficiency decreases with increasing an external magnetic field.

Since many organic molecules, which are potentially interesting for DNP as polarizers, are not soluble in water, the Overhauser DNP analysis has been done for toluene solutions. Also nonpolar solvents, in contrast to water, suffer less from dielectric losses and this allows using larger volume of the investigated samples. These experiments have demonstrated higher DNP efficiency of nitroxide radicals in toluene solution than in aqueous solution at low radical concentration. The reason is higher Heisenberg spin exchange rate between different hyperfine EPR transitions due to lower viscosity of toluene.

The theoretical model of the relaxation dynamic for the electron-nuclear hyperfine coupled system with $\mathrm{S}=1 / 2$ and $\mathrm{I}=1$ has been analytically solved and the analytical expressions for the saturation factors of the individual hyperfine lines have been obtained. The electron-electron double resonance (ELDOR) method has been extended for the experimental measurements of the saturation factors and the polarization recoveries for radicals with more than 2 hyperfine lines. The series of experiments have demonstrated that the DNP enhancement increases with increasing of molecular size and does not depend on the nitroxide radical concentration in the case of dominant nitrogen nuclear relaxation between hyperfine EPR transitions.

Several nitroxide derivatives with the covalently linked fullerene $\mathrm{C}_{60}$, as the dye with high quantum yield of the triplet state, have been synthetized. The transient EPR experiments of the photoexcited fullerene-nitroxide derivatives have demonstrated the feasibility to generate high non-equilibrium electron spin polarization by light. The photoexcited electron polarization, if successfully transferred to solvent nuclei, may provide a new method to perform DNP in liquids without MW irradiation. 


\section{Contents}

1. Introduction 1

1.1. Polarization in Magnetic Resonance ................................................... 1

1.2. Dynamic nuclear polarization (DNP) .............................................. 5

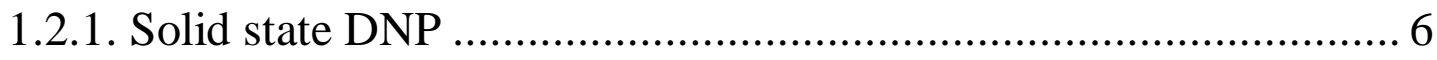

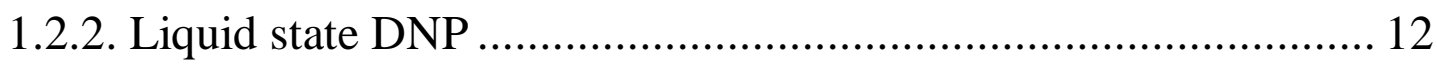

1.2.4. Current applications of liquid-state DNP …............................... 24

1.2.5. Polarizing agents for DNP …................................................. 26

1.3. Dynamic electron polarization (DEP) for DNP with photoexcited

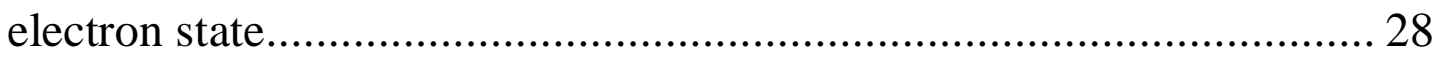

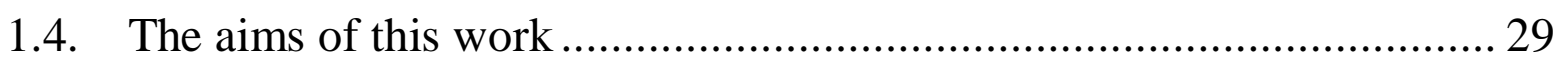

2. Theoretical background $\quad 31$

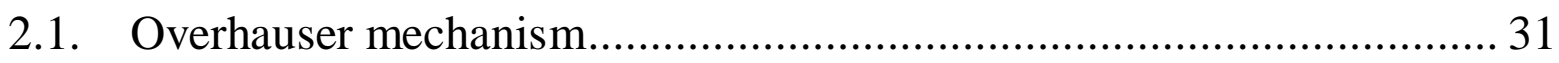

2.1.1. Saturation factor of EPR lines: relaxation effects between

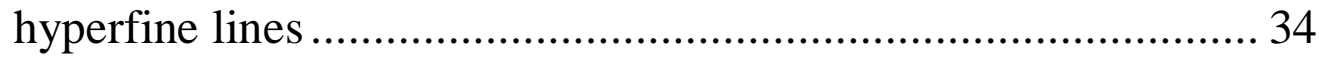

2.1.2. Coupling factor: relaxations in paramagnetic solution ................ 41

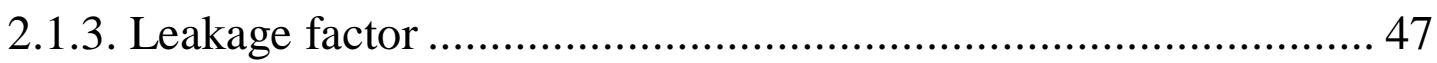

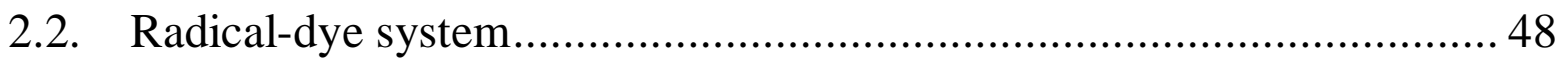

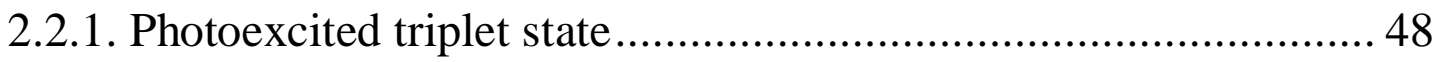

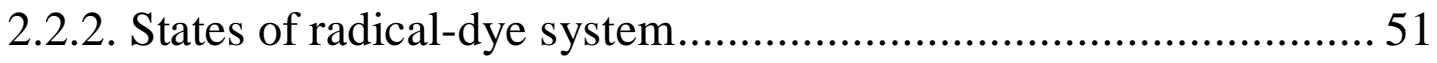

2.2.3. Reversed quartet mechanism ................................................. 53

3. Materials and methods $\quad 56$

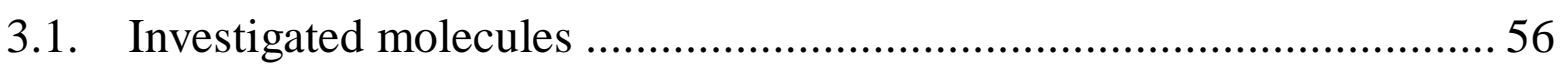

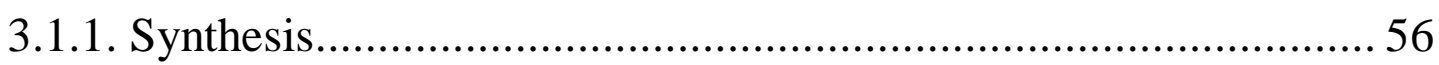

3.2. Rotational correlation time …........................................................ 59

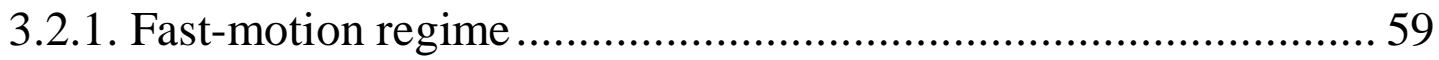

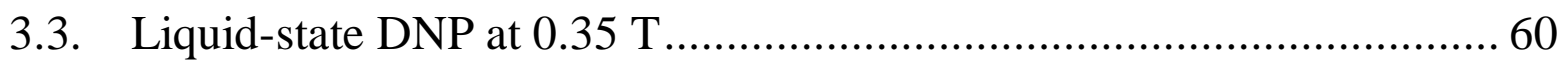

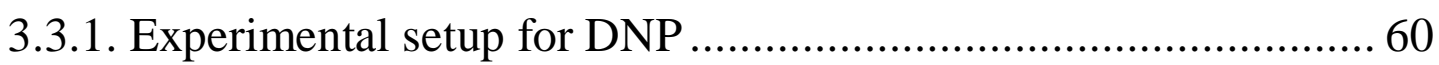

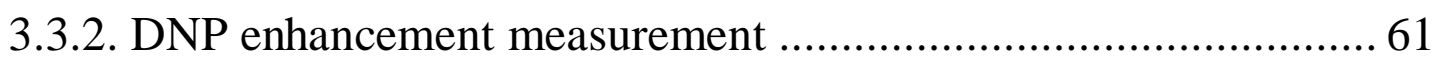

3.4. Electron-electron double resonance (ELDOR) ......................................62 62

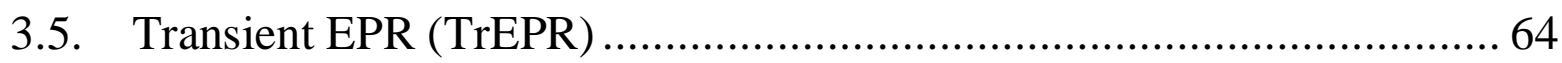

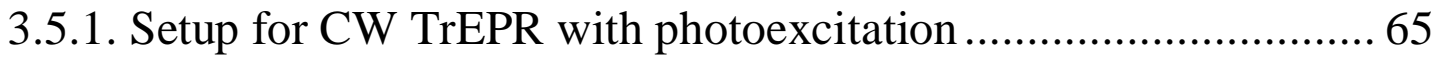

3.5.2. Continuous wave transient EPR (CW TrEPR) ............................ 65

3.5.3. Time evolution of EPR signal by pulsed EPR …......................... 67 
4. Comparative DNP studies in water and toluene: effect of solvent

4.1. EPR of TEMPONE- $\mathrm{D}_{16^{-}}{ }^{15} \mathrm{~N}$ in toluene solution at $0.3 \mathrm{~T}$...................6 69

4.2. DNP with TEMPONE- $\mathrm{D}_{16^{-}}{ }^{15} \mathrm{~N}$ in toluene solution at $0.3 \mathrm{~T}$................. 70

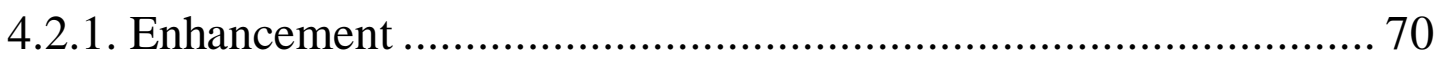

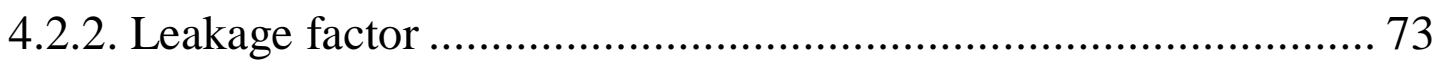

4.2.3. Saturation factor: the role of Heisenberg spin exchange.............. 74

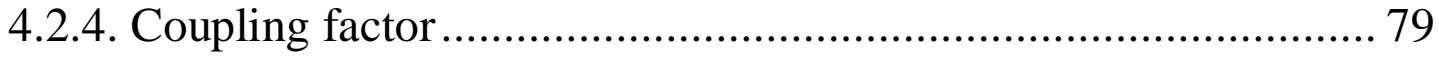

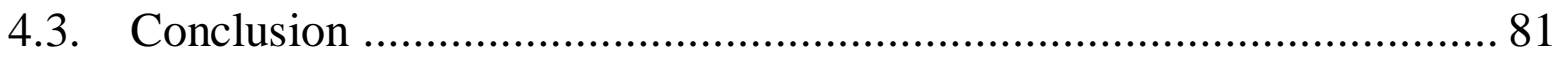

5. DNP with fullerene-nitroxide derivatives: effect of polarizer's size 82

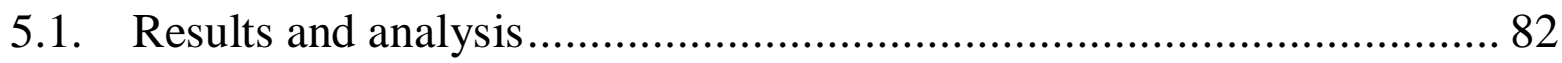

5.1.1. Determination of rotational correlation time ............................. 82

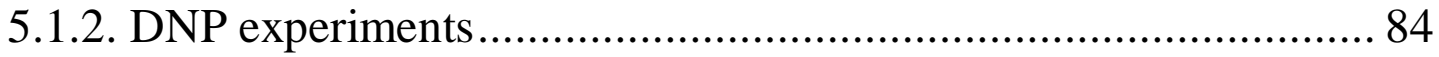

5.1.3. Saturation factor: ELDOR measurements .................................. 86

5.1.4. Discussion: the role of nitrogen nuclear relaxation ..................... 89

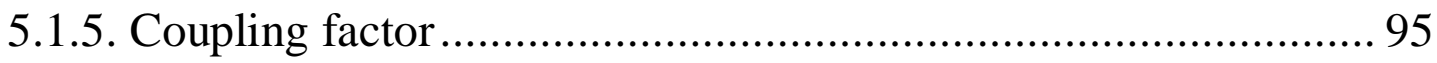

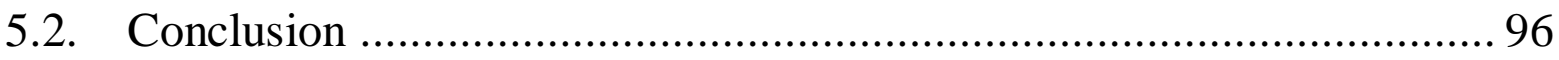

6. DEP with fullerene-nitroxide derivatives 97

6.1. Transient EPR of fullerene-nitroxide derivatives ............................... 97

6.2. Perspectives of DNP with photoexcited fullerene-nitroxide systems .. 101

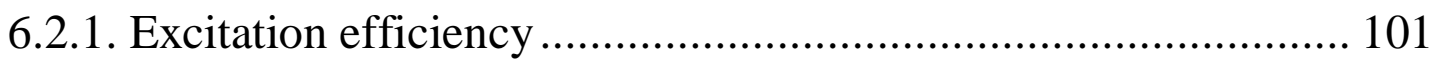

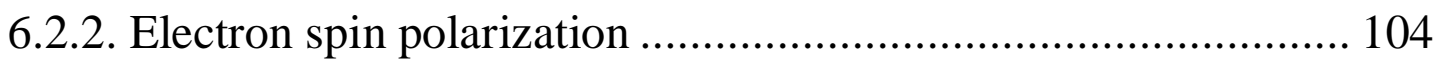

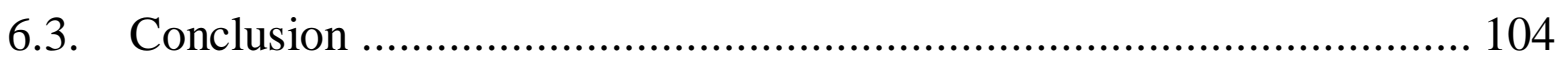

$\begin{array}{ll}\text { 7. References } & 106\end{array}$

$\begin{array}{ll}\text { List of Abbreviations } & 121\end{array}$

$\begin{array}{ll}\text { List of Figures } & 123\end{array}$

$\begin{array}{ll}\text { List of Tables } & 127\end{array}$

$\begin{array}{ll}\text { 8. Appendix } & 128\end{array}$

$\begin{array}{ll}\text { Curriculum Vitae } & 131\end{array}$ 


\section{Introduction}

\section{Introduction}

Nuclear magnetic resonance spectroscopy and imaging (NMR/MRI) are modern versatile techniques which are used for the non-destructive identification and structural characterization of materials, biochemical systems and diagnostic in medicine. The main disadvantage of these techniques is the relatively low signal-to-noise ratio and resolution of nuclei at ambient conditions (room temperature and liquid state) due to the small magnetic moment of nuclear spins. Therefore, they usually require large volume and concentration of the observed sample and long measurement time.

The ordinary ways to improve NMR sensitivity deal with several instrumental innovations such as superconducting magnets for high-field experiments $(23.5 \mathrm{~T})$ and cryogenic probes. The Fourier transformation (FT) methodology (pulsed NMR) eliminates the need of frequency scanning and enabled the fast accumulation of multiple transients in a short time. Suitable materials of receiver coil minimize magnetic field distortions. The signal filters help to eliminate an aliased noise.

\subsection{Polarization in Magnetic Resonance}

For systems with spin value $1 / 2$ (for example, nuclei ${ }^{1} \mathrm{H},{ }^{13} \mathrm{C},{ }^{15} \mathrm{~N}$ ) the energy difference of non-degenerate spin states is $\Delta E=\hbar \gamma B$, where $\hbar$ is Planck constant, $\gamma$ is the gyromagnetic ratio of an investigated spin system and $B$ is the external magnetic field (Fig. 1.1). 


\section{Introduction}

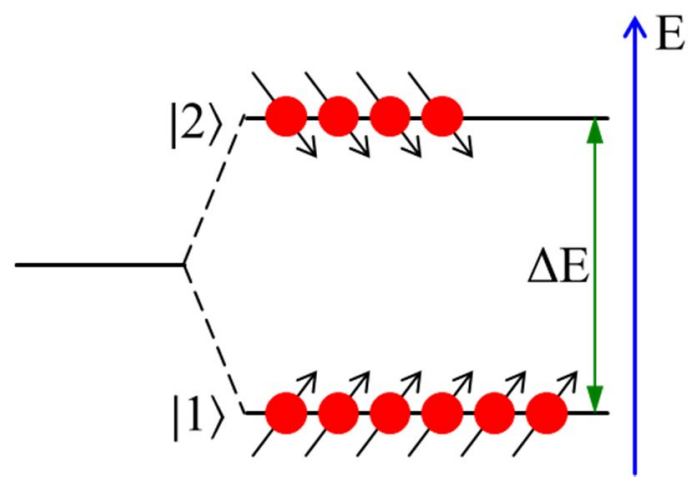

Figure 1.1: Representation of the splitting of spin states $(|1\rangle=|-1 / 2\rangle$ and $|2\rangle=|+1 / 2\rangle)$ due to an external magnetic field.

The intensity of NMR signal is proportional to the population difference $\left(n_{1}-n_{2}\right)$ of spin states. The polarization $P$ is commonly defined as:

$$
P=\frac{n_{1}-n_{2}}{n_{1}+n_{2}}
$$

$P$ can have values between -1 and 1 . It is useful to keep in mind that $P$ is often expressed in percentages $(P \times 100)$.

According to Boltzmann distribution the polarization $P$ can be as

$$
P=\frac{n_{1}-n_{2}}{n_{1}+n_{2}}=\tanh \left(\frac{\Delta E}{2 k T}\right)=\tanh \left(\frac{\mathrm{h} \gamma B}{2 k T}\right)
$$

where $k$ is Boltzmann constant, $T$ is the absolute temperature. The small $\gamma$ of nucleus yields small Boltzmann polarization at room temperature and low magnetic field (Fig. 1.2). At the largest NMR magnet available today with $B \approx 25 \mathrm{~T}$, which corresponds to the proton Larmor frequency $\sim 10^{3} \mathrm{MHz}$, the polarization is less than $0.01 \%$ at room temperature and around $3 \%$ at $1 \mathrm{~K}$ (Fig. 1.2). 


\section{Introduction}

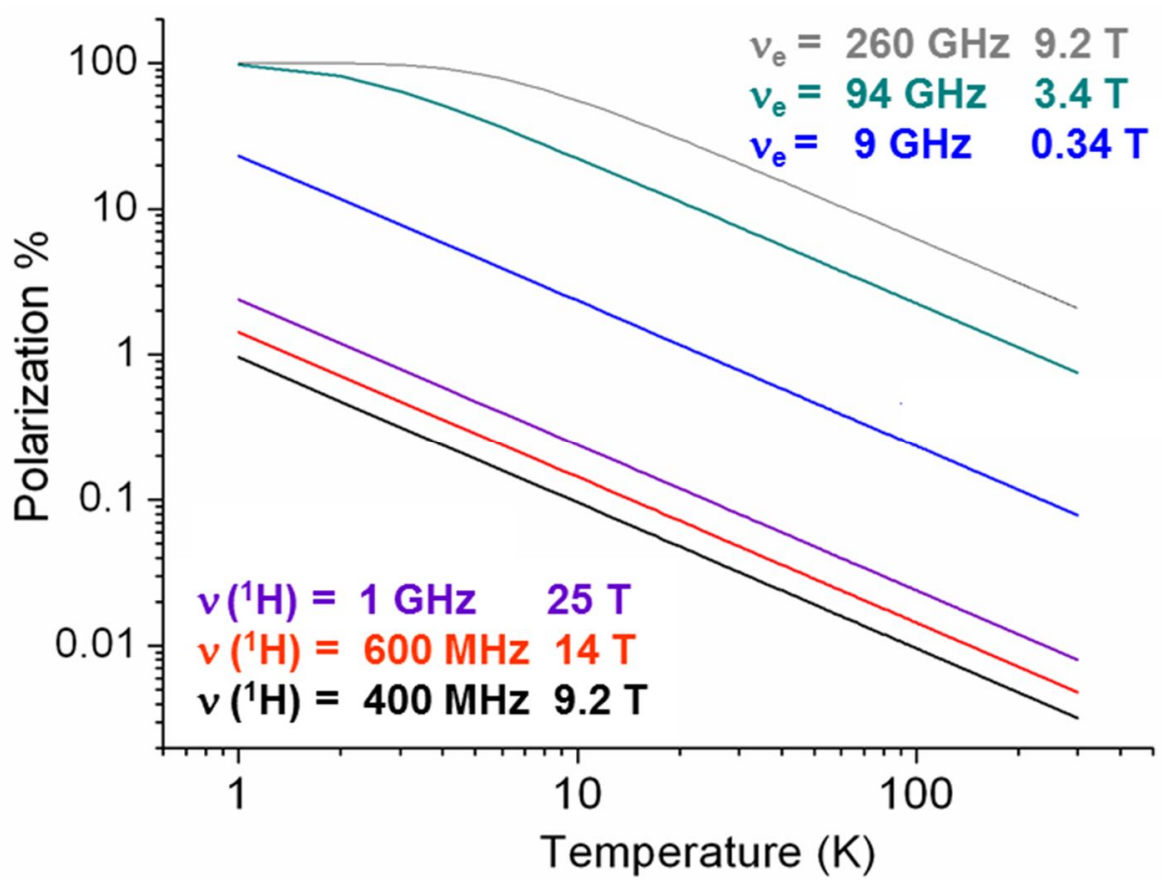

Figure 1.2: Polarization of electron spins (e) and proton $\left({ }^{1} \mathrm{H}\right)$ as function of temperature at various external magnetic field strength values.

Over the last several decades the improvement of NMR/MRI sensitivity, resolution and signal intensity is a progressive field of researches. The most interesting strategy is the search and development of methods which are able to raise spin polarization far from the thermal equilibrium value.

In 1953, Overhauser proposed the possibility of spin polarization transfer from unpaired electrons to neighbour nuclei in metals in result of saturating the electron resonance transitions ${ }^{1,2}$. Later Carver and Slichter experimentally demonstrated this effect (Overhauser effect, OE) in alkali metals and liquids ${ }^{3,4}$. In 1955 Abragam reported a theoretical model of Overhauser mechanism in non-metals ${ }^{5}$. In 1957 Bennett and Torrey estimated both value and relative sign of an enhancement in liquids and its dependence on quality of a saturation of an irradiated EPR transition ${ }^{6}$. The mechanism, called Overhauser effect, requires the free motion of the unpaired electrons which can exist in liquids or in metals with conducting band. The NMR enhancement by OE can have maximum value of $\gamma_{e} / \gamma_{I} 660$ for ${ }^{1} \mathrm{H}$ and $2.6 \times 10^{3}$ for ${ }^{13} \mathrm{C}$. Hausser and Stehlik described the theoretical framework for Overhauser effect in liquids ${ }^{7}$. 


\section{Introduction}

In 1957, Jeffries found a mechanism of nuclear polarization in solids governed by the saturation of forbidden transitions in electron-nuclear coupled systems. It was called the solid effect (SE). Kessenikh in 1963, and then Hwang and Hill, and Wollan described another effect, the cross effect (CE), in solid state systems ${ }^{8-13}$. In 1968, Borghini presented a spin-temperature model of nuclear spin polarization (thermal mixing, TM) at low temperature ${ }^{14}$. Later he showed the experimental evidence for this model ${ }^{15}$. In 1997, Griffin et al. demonstrated nuclear polarization experiment with biomolecules in a frozen solution using magic-angle spinning nuclear magnetic resonance (MAS) ${ }^{16}$.

Spin polarization can also be created by optical pumping circular polarized light on systems with unpaired electrons. It is realized in alkali metal (typically $\mathrm{Rb}$ ), free radical and semiconductors ${ }^{17-19}$. In 1960, Bouchiat et al. demonstrated an experiment where nuclei of ${ }^{3} \mathrm{He}$ were polarized by means of spin exchange between ${ }^{3} \mathrm{He}$ and vapours of $\mathrm{Rb}$ irradiated by circularly polarized light ${ }^{17}$. Thus spin polarization by optical pumping consists of irradiation alkali metal and, subsequently, a transfer polarization to noble gas. In 1985, Happer et al. improved the spin exchange efficiency for transfer polarization from alkali metal vapours to a noble gas $\left({ }^{129} \mathrm{Xe}\right)$ using three-body collision mechanism (including nitrogen $\mathrm{N}_{2}$ ) which leads to a formation of alkali-metal-noble-gas van der Waals molecules ${ }^{20}$. Additionally to ${ }^{3} \mathrm{He}$ and ${ }^{129} \mathrm{Xe}$ the optical pumping hyperpolarization experiment was demonstrated with ${ }^{83} \mathrm{Kr}$ by Meersman and coworkers in $2005{ }^{21}$. The hyperpolarization method based on the optical pumping and ${ }^{129} \mathrm{Xe}$ has become very popular and useful application in magnetic resonance imaging (MRI) ${ }^{22-26}$. It can be used in the gas phase for direct Xe application or for hyperpolarization observed nuclei through Overhauser effect ${ }^{23,24}$.

In 1967, Bargon et al. observed the appearance of nuclear magnetic resonance emission signal in the process of rapid chemical reactions of organic radicals ${ }^{27}$. Independently, Ward et al. reported experimental work, in which high positive and negative proton polarization in simple olefins were found during a fast reaction with organometallic compounds ${ }^{28}$. After one year Cocivera detected an enhanced NMR signal of protons of anthroquinone diluted in perfluorobenzene when the solution at room temperature was optically excited by light $(300-400 \mathrm{~nm})^{29}$. That phenomena was called 


\section{Introduction}

photochemically induced dynamic nuclear polarization (photo-CIDNP). Its principle is based on a generation nuclear polarization of a molecule of interest in result of interaction with a photoexcited dye as a polarizer component. In 1978 Kaptein and coworkers reported photo-CIDNP experiment as a surface probe for proteins analysis in a native state ${ }^{30}$. The nuclear polarization of the proteins was generated by cycling a chemical interaction between photoexcited dye and accessible amino acids. Flavin was used as a dye and tryptophan, tyrosine, histidine was found as amino acids with hyperpolarized signals. Hore and coworkers demonstrated applications of photo-CIDNP for biomolecules and technical improvements for qualitative experiments ${ }^{31-34}$. PhotoCIDNP does not require the presence of stable radicals and freeze-melting cycles for an experimental realization.

Bowers and Weitekamp proposed in 1986 and proved in 1987 that the formation molecules by addition of diatomic hydrogen $\left(\mathrm{H}_{2}\right)$ in the para nuclear spin state can lead to appearance of a hyperpolarized NMR signal ${ }^{35}$, 36. The para-hydrogen induced polarization (PHIP) method is based on the observed mechanism. This method was applied to hyperpolarization of unsaturated organic molecules through hydrogenation reaction with para-hydrogen and led to hyperpolarization near $100 \%{ }^{37}$. The PHIP mechanism was found as perspective for studies of transition metal complexes ${ }^{38}$. In 2009, Duckett and coworkers demonstrated that the PHIP can be applied for hyperpolarization without hydrogenation of molecules of interest. The metal centre was used to facilitate reversible approach for polarization transfer from para-hydrogen to a substrate under study ${ }^{39}$.

\subsection{Dynamic nuclear polarization (DNP)}

Dynamic nuclear polarization (DNP) provides a mean to enhance the NMR polarization and to improve the performance of NMR based techniques. Owing to the large gyromagnetic ratio of unpaired electron, its polarization reaches high value compared to nuclear one. The DNP method relies on polarization transfer from electron spins to surrounding nuclei and can enhance the nuclear spin polarization by orders of 


\section{Introduction}

magnitude. The DNP mechanisms depend on temperature and state of aggregation of the observed system. DNP experiments can be classified by the polarization mechanisms and the technical setups (continuous wave versus pulsed) of the experiment. Fig. 1.3 shows the energy level diagram of the electron-nuclear coupled system with the responsible transition probabilities.
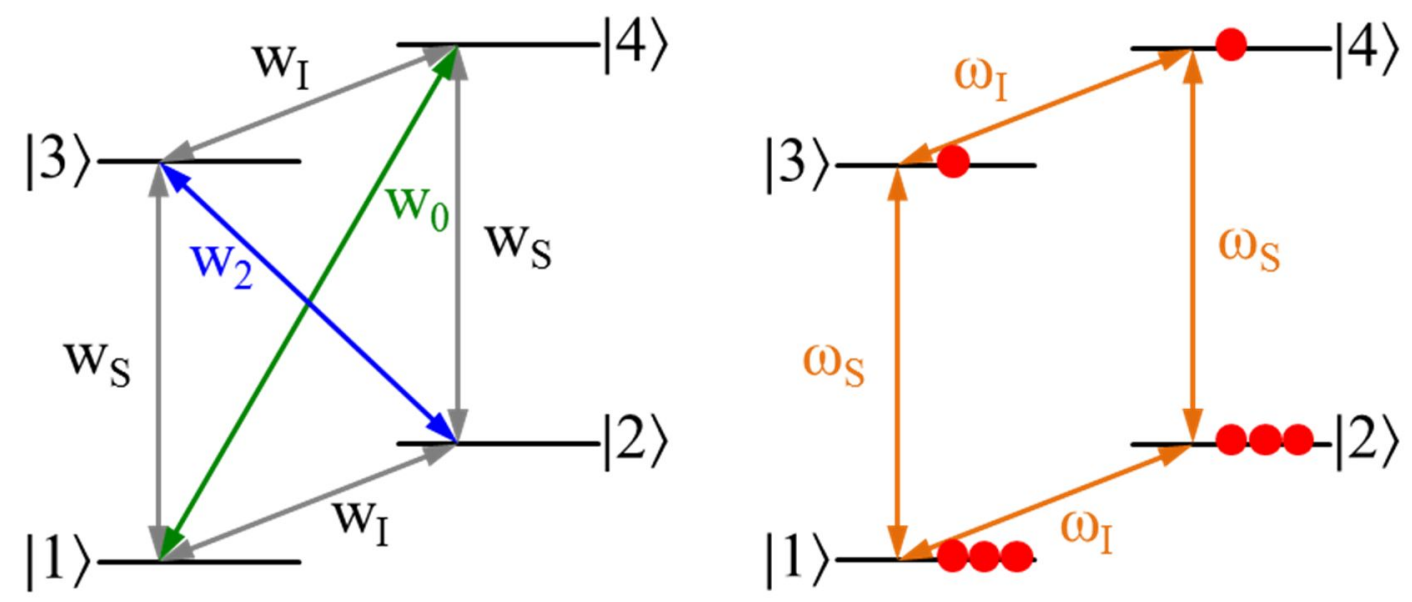

Figure 1.3: Right: Energy level diagrams and transition rates $\left(w_{S}\right.$ and $w_{I}$ are the probabilities of EPR and NMR transitions, respectively; $w_{0}$ and $w_{2}$ are the rates of zero and double quantum transitions, respectively); Left: Population distribution and Larmor frequencies of electron and nucleus ( $\omega_{S}$ and $\omega_{I}$, respectively).

1.2.1. Solid state DNP. DNP mechanisms in solids are presently classified into following five types as the solid effect (SE) ${ }^{40-42}$ and the cross effect (CE) ${ }^{8,10,11}$ in continuous DNP experiment and the integrated solid effect (ISE) ${ }^{43,44}$, dressed-state solid effect (DSSE) ${ }^{45,46}$ and NOVEL ${ }^{47,48}$ in pulsed DNP experiment.

Solid effect. In the solid effect (SE) microwave (MW) irradiation on the zero and/or double quantum transitions $(|1\rangle \leftrightarrow|4\rangle$ and $|2\rangle \leftrightarrow|3\rangle$, respectively) leads to nuclear polarization (Fig. 1.4) ${ }^{42}$. The saturation of the zero quantum transition at frequency $\omega_{e}+\omega_{I}$ results in negative DNP enhancement whereas the double quantum transition case at frequency $\omega_{e}-\omega_{I}$ produces positive enhancement. 


\section{Introduction}
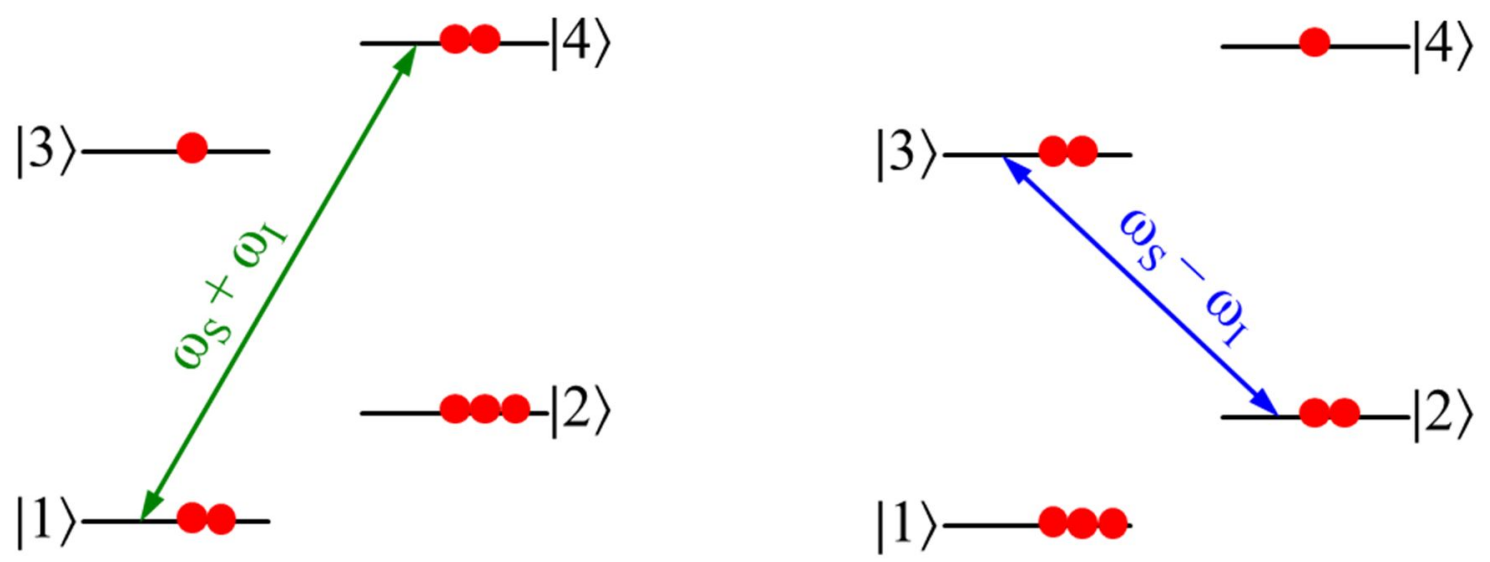

Figure 1.4: The MW irradiation of the forbidden transitions equalizes the populations of correspondent spin states, which produces negative (left) or positive (right) DNP enhancement.

The SE is observed at low temperature in solids and requires a paramagnetic species with a homogenous linewidth $\delta$ and an inhomogeneous breadth $\Delta$ of the EPR spectrum smaller than the nuclear Larmor frequency $\omega_{I}\left(\delta, \Delta<\omega_{I}\right)$. The latter condition is needed to saturate only one of the forbidden transitions. Otherwise, in result of the simultaneous saturation of both transitions, the two processes of nuclear population changing compensate each other and lead to partial or complete reduction of the polarization effect. This case is known as the differential solid effect (DSE). Since the DNP enhancement of $\mathrm{SE}$ is inverse proportional to square of external magnetic field the efficiency of this method is very small at high magnetic field,

$$
\varepsilon_{S E} \sim \frac{\gamma_{S}}{\gamma_{I}}\left(\frac{B_{1 S}}{B}\right)^{2} \frac{N_{S}}{\delta} T_{1 I},
$$

where $B_{1 S}$ is the MW field strength, $N_{S}$ is the electron concentration and $T_{1 I}$ is the nuclear spin-lattice relaxation time ${ }^{49-51}$.

Cross effect. The $\mathrm{CE}$ is based on allowed transitions and involves the dipolar interaction of electron spin packets in an inhomogeneously broadened EPR line ${ }^{8-11}$. Griffin et al. proposed that CE can be defined as three-spin system (two electrons and one nucleus), involving the interaction between two dipolar coupled electrons with Larmor frequencies $\left(\omega_{S 1}\right.$ and $\left.\omega_{S 2}\right)$ which satisfy the relation ${ }^{52}$

$$
\omega_{S 1}-\omega_{S 2}=\omega_{I}
$$




\section{Introduction}

The CE occurs when the inhomogeneous EPR linewidth is larger than the nuclear Larmor frequency $\left(\omega_{I}<\Delta\right)$ what makes the electron Larmor frequency effectively satisfy the relation (1.4). At the same time the homogeneous width must be smaller than the nuclear Larmor frequency $\left(\delta<\omega_{I}\right)$. The CE is the dominant mechanism of nuclear polarization if the inhomogeneous line width is a result of the $g$ anisotropy and the electrons are weakly coupled through the relaxation of zero and/or double quantum transitions. The cross effect is very effective when a biradical is used as polarizing agent. It allows achieving the condition for the homogeneous line width. The DNP enhancement of CE is linear inverse proportional to the magnetic field

$$
\varepsilon_{C E} \sim \frac{\gamma_{S}}{\gamma_{I}} \frac{B_{1 S}^{2}}{B} \frac{N_{S}^{2}}{\delta^{2}} T_{1 I}
$$

which makes the CE mechanism more efficient at high magnetic field than SE (1.3). As result, the $\mathrm{CE}$ is most commonly used mechanism for to obtain DNP enhancements at high magnetic field.

Influence of spin diffusion. Spin diffusion (SD) is a mechanism of magnetization exchanging of dipolar coupled spins among each other ${ }^{53,54}$. In SE and CE the spin polarization is transferred from electrons only to the nuclei, which are hyperfine coupled with the electrons whereas the NMR signal arises from the bulk nuclei. Most of the bulk nuclei are not hyperfine coupled with the electrons due to the large distance or any other reasons. The SD process transfers spin polarization from the nuclei coupled with electrons to the bulk nuclei. In DNP experiment with a solid sample of dissolved proteins the polarization is transferred from the nuclei of the solvent to nuclei of the proteins by means of SD.

In 1962, Hartmann and Hahn reported a pulsed DNP experiment based on the coherent polarization transfer from one spin system to another one $e^{55}$. The mechanism was latter called as Hartmann-Hahn cross polarization (HHCP). As it was shown the HHCP does not depend on increasing magnetic field that makes it be more efficient at high magnetic field NMR. 


\section{Introduction}

The HHCP is nowadays a very useful technique for solid-state NMR where the spin polarization of one nucleus (usually ${ }^{1} \mathrm{H}$ ) can be transferred to other nuclei (such as ${ }^{13} \mathrm{C}$, $\left.{ }^{15} \mathrm{~N}\right){ }^{56,57}$. The HHCP process requires the fulfillment of the rotating frame HartmannHahn matching condition for applied RF magnetic strengths $\left(\omega_{1}\right)$ of two irradiated nuclei $\left(I_{1}\right.$ and $\left.I_{2}\right)$

$$
\omega_{1}\left(I_{1}\right)=\omega_{1}\left(I_{2}\right)
$$

In solid-state DNP experiment RF field strength is strong enough to cover the entire NMR spectrum and efficiently spin-lock the both spin species. Thus the Hartmann-Hahn condition (1.6) is satisfied.

In DNP experiment the large inhomogeneous width of the EPR spectrum can exceed several hundred megahertz. It means that MW field strength is one to two orders of magnitude smaller than RF field strength. As a result, the modification of MW and RF field strength cannot reach the Hartmann-Hahn relation (1.6) for electron-nuclear cross polarization. Therefore, off resonance effect in EPR must be considered.

The NMR experiment with HHCP is typically performed by polarization transfer from high- $\gamma$ spins to low- $\gamma$ spins. According to thermodynamic theory the signal enhancement in HHCP is

$$
\varepsilon=\frac{\gamma_{1}}{\gamma_{2}} \frac{1}{1+N_{2} / N_{1}}
$$

where $N_{l}$ and $N_{2}$ are the concentrations of spins with high $\left(\gamma_{1}\right)$ and low $\left(\gamma_{2}\right)$ gyromagnetic ratios, respectively ${ }^{58}$. The high efficiency of HHCP requires that the concentration of the high- $\gamma$ spins is much larger than the concentration of the low- $\gamma$ spins $\left(N_{1} \gg N_{2}\right)$. In this case the enhancement is around $\gamma_{1} / \gamma_{2}$ and this full ration can be transferred. However, in DNP experiment the unpaired electron spins (as high- $\gamma$ compound) concentration is about four orders of magnitude less than the nuclear spin concentration. This leads to enhancement $\varepsilon<1$ and only small part of the nuclei can be polarized. Since the electron relaxation $\left(T_{I S}\right)$ is much shorter than the nuclear one $\left(T_{I I}\right)$ the applied MW pumping, as multiple HHCP contact, allows to improve the DNP efficiency of HHCP mechanism. 


\section{Introduction}

Integrated solid effect. As described above, if a homogenous linewidth $\delta$ is much larger than the nuclear Larmor frequency $\left(\delta>\omega_{I}\right)$ it makes the SE inefficient since simultaneously saturation of both forbidden transitions at a frequency $\omega_{S} \pm \omega_{I}$ compensate each other. However Henstra et al. introduced that if a forbidden transition can be selectively inverted prior to saturation of an allowed transition it allows for overcoming the problem of the low DNP efficiency of SE with high homogenous broadening of EPR $\operatorname{line}^{43,44}$. This approach is known as the integrated solid effect (ISE).

Electron-nuclear cross polarization. Electron-nuclear cross polarization (eNCP) was first introduced by Weis et al. ${ }^{45,46}$. This DNP mechanism is based on simultaneous near resonant MW and RF irradiation. The selective excitation of the dressed state transitions creates the polarization of a core nucleus in nuclear laboratory frame and leads to transferring this polarization to surrounding nuclei even in the case of the absence of nonsecular hyperfine coupling terms.

Due to the limited MW power at high frequency the allowed EPR transitions cannot be excited with the same MW field strength because the resulting effective fields $\omega_{1 S}^{a, e f f}$ and $\omega_{1 S}^{b, e f f}$ have unequal influence (Fig. 1.5). The same situation is with RF field strength and nuclei. As result, the problem is an impossibility of the transformation into the electron/nuclear frame with a single angle $\theta$.
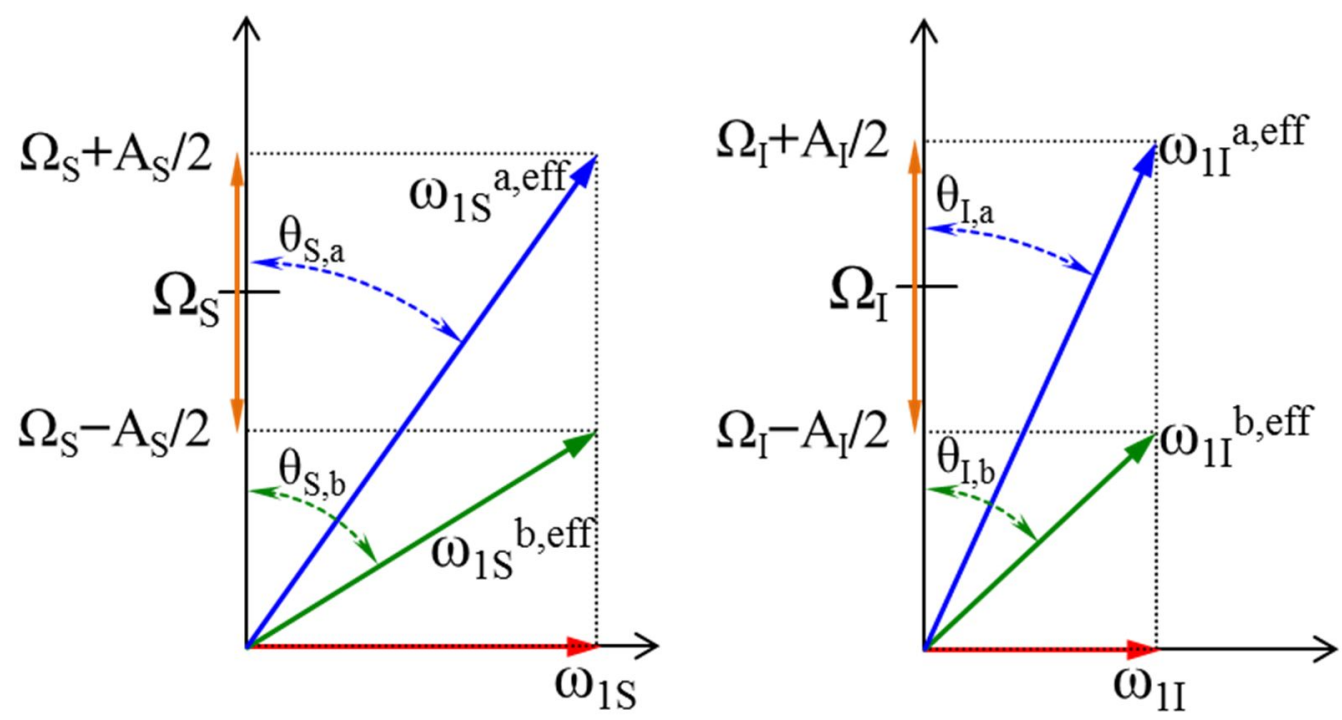

Figure 1.5: Illustration of electron (left) and nuclear (right) spin effective fields. 


\section{Introduction}

The matching condition for eNCP, as cross polarization with four effective fields, is

$$
\begin{aligned}
& \sqrt{\left(\Omega_{S}+A_{S} / 2\right)^{2}+\omega_{1 S}^{2}}+\sqrt{\left(\Omega_{S}-A_{S} / 2\right)^{2}+\omega_{1 S}^{2}}= \\
& = \pm\left[\sqrt{\left(\Omega_{I}+A_{I} / 2\right)^{2}+\omega_{1 I}^{2}}+\sqrt{\left(\Omega_{I}-A_{I} / 2\right)^{2}+\omega_{1 I}^{2}}\right],
\end{aligned}
$$

where $\Omega_{S}$ and $\Omega_{I}$ are the resonant offsets, $A_{S}$ and $A_{I}$ are hyperfine coupling constants of electron and nucleus, respectively, $\omega_{1 S}$ and $\omega_{1 I}$ are $\mathrm{MW}$ and RF field strengths, respectively. In NMR-CP at MW irradiation $\left(\Omega_{S}=0\right)$ and the arbitrary hyperfine coupling the simplified matching relation is

$$
\Omega_{I} \approx \sqrt{\omega_{1 S}^{2}+\left(A_{S} / 2\right)^{2}-\omega_{1 I}^{2}} \approx \sqrt{\omega_{1 S}^{2}+\left(A_{S} / 2\right)^{2}} .
$$

This is more general Hartmann-Hahn condition of hyperfine selective polarization transfer. In the case $\Omega_{I} \approx \omega_{1 S}$ only nuclei with weak coupling can be polarized.

The eNCP theory has been adapted for the case of larger hyperfine couplings in a new experiment called CP-ENDOR recently proposed by Rizzato and Bennati ${ }^{59}$. In these experiments the nuclei coupled with electron spins are pre-polarized in order to provide a more efficient electron-nuclear double resonance (ENDOR) detection.

NOVEL. In 1980-s Brunner et al. and Henstra et al. introduced independently the experiments with nuclear spin orientation via electron spin locking (NOVEL) ${ }^{47,48}$. In NOVEL experiment the electron spin magnetization is locked in the electron rotating frame by electron spin locking sequence. If the field strength of locking pulse $\left(\omega_{1 S}\right)$ satisfies the relation

$$
\omega_{1 S}=\omega_{I}
$$

then the Hartman-Hahn condition of the electron-nuclear system in rotating frame or lab frame is fulfilled and thus the polarization can be transferred from electron to nuclei. Henstra et al. measured the dependence of the enhancement on spin locking time in NOVEL experiment where the electron polarization transfer from pentacene in a 


\section{Introduction}

photoexcited triplet state, as a guest molecule, to ${ }^{1} \mathrm{H}$ of naphthalene, as a host crystal ${ }^{60}$. The maximum achieved value of the proton enhancement was around 220 .

Mostly the NOVEL experiments are performed at low magnetic field $(9 \mathrm{GHz} \mathrm{MW}$ frequency) ${ }^{60-63}$. The application of this method at high field requires MW source with much higher field strength. The development the high frequency MW source like klystron ${ }^{64}$ and gyrotron ${ }^{65}$ with high power (MW field strength) can make the NOVEL method be more useful because it provides a simple polarization scheme without requirement of additional RF pulses.

1.2.2. Liquid state DNP. The only mechanism which governs DNP in liquid state is based on Overhauser effect (OE).

Overhauser effect. The OE DNP mechanism results from a relaxation process which relies on simultaneously flipping electron and nuclear spins. These relaxation processes are based on the time-dependent interactions between electrons and nuclei. In liquids, they are governed by molecular translational and rotational motions in the dipolar case and by chemical exchanges and fast relaxation in the scalar case ${ }^{66}$. In solids, the $\mathrm{OE}$ relaxation process requires presence of mobile electrons which are offered by the conduction band of a metal ${ }^{3}$ or one-dimensional organic conductors ${ }^{67}$. In 2014, Griffin et al. have observed the $\mathrm{OE}$ in insulating solids ${ }^{68}$. Also the successful establishment of $\mathrm{OE}$ requires the condition $\omega_{S} \cdot \tau_{c}<1$ (where $\tau_{c}$ is a rotational correlation time of paramagnetic species) which is difficult to satisfy when $\omega_{S}$ becomes large. Thus the efficiency of OE decreases at high magnetic field. However, Overhauser DNP is the only and very successful method for the direct polarization in liquids.

When the allowed EPR transitions are saturated the relaxations of the forbidden transitions $\left(w_{0}\right.$ and $\left.w_{2}\right)$ induce changes in the nuclear spin population and the enhanced NMR signal is observed. The enhancement of the NMR signal arises due to the relaxation processes where electron and nuclear spins are reversed simultaneously in the opposite or in the same directions (zero and double quantum transitions, respectively). According to the kinetic equation based on the treatment of the OE described by Solomon ${ }^{69}$ 


\section{Introduction}

$$
\frac{d}{d t}\left\langle I_{z}\right\rangle=-\left(w^{0}+2 w_{I}+w_{0}+w_{2}\right)\left(\left\langle I_{z}\right\rangle-I_{z}^{B}\right)+\left(w_{2}-w_{0}\right)\left(\left\langle S_{z}\right\rangle-S_{z}^{B}\right)
$$

the DNP enhancement $\varepsilon$ can expressed as

$$
\varepsilon=1-\xi \cdot f \cdot s \cdot \frac{\left|\gamma_{S}\right|}{\gamma_{I}},
$$

where $\xi, f$ and $s$ are coupling, leakage and saturation factors, respectively,

$$
\xi=\frac{w_{2}-w_{0}}{2 w_{I}+w_{0}+w_{2}}, \quad f=\frac{2 w_{I}+w_{0}+w_{2}}{2 w_{I}+w_{0}+w_{2}+w^{0}}, \quad s=\frac{S_{z}^{B}-\left\langle S_{z}\right\rangle}{S_{z}^{B}} .
$$

The leakage factor characterizes the contribution of the electron towards the nuclear relaxation and can have values form 0 (no radical contribution) to 1 (relaxation controlled by radical). The electron contribution depends on the radical concentration. For systems with nitroxide radicals as polarizers the leakage factor reaches a value of 1 at concentration of $20 \mathrm{mM}$.

The saturation factor, as degree of the electron transition saturation, depends on the MW power and the electron spin-lattice relaxation ${ }^{70}$. However, saturation behavior becomes much more complicated if the polarizer agent has several electron transition lines due to hyperfine interaction with a nucleus and it is not possible to irradiate simultaneously all EPR lines. It corresponds to the nitroxide radical where hyperfine interaction with nitrogen leads to appearance two $\left({ }^{15} \mathrm{~N}\right)$ or three $\left({ }^{14} \mathrm{~N}\right)$ lines. Additionally to the electron relaxation the system with several hyperfine lines include Heisenberg spin exchange (HE) and nuclear (nitrogen in the case of nitroxide radicals) spin-lattice relaxation as two important mechanisms of interaction between EPR transitions. The first theoretical model describing that behavior with HE mechanism was proposed by Bates and Drozdoski ${ }^{71}$. Armstrong extended it taking into account also the nuclear relaxation effect ${ }^{72}$. Türke and Bennati used pulsed electron-electron double resonance (ELDOR) as the approach for the experimental determination of the saturation of all EPR transition when only one line is irradiated ${ }^{73}$. The ELDOR experiments with TEMPONE-D ${ }_{16}{ }^{15} \mathrm{~N}$ demonstrated a strong dependence of the second line saturation on the radical 


\section{Introduction}

concentration that means the predomination of the HE mechanism. Therefore, the complete saturation of the TEMPO radicals requires high concentration.

The coupling factor estimates a nature of interaction between a polarizer and nuclei of a solvent. The interaction consists of dipolar and scalar terms ${ }^{69}$. Hausser used a semiclassical approach for description the relaxation mechanisms through Lorantz-shape spectral density function for rotational diffusion ${ }^{69,74}$. Müller-Warmuth and coworkers extended the model including the translational and pulse diffusions ${ }^{75}$. Hwang and coworkers developed the translational diffusion model according to force-free pair correlation functions ${ }^{76}$. The coupling factor is mainly responsible for field dependence of the DNP efficiency governed by Overhauser mechanism (Fig. 1.6).

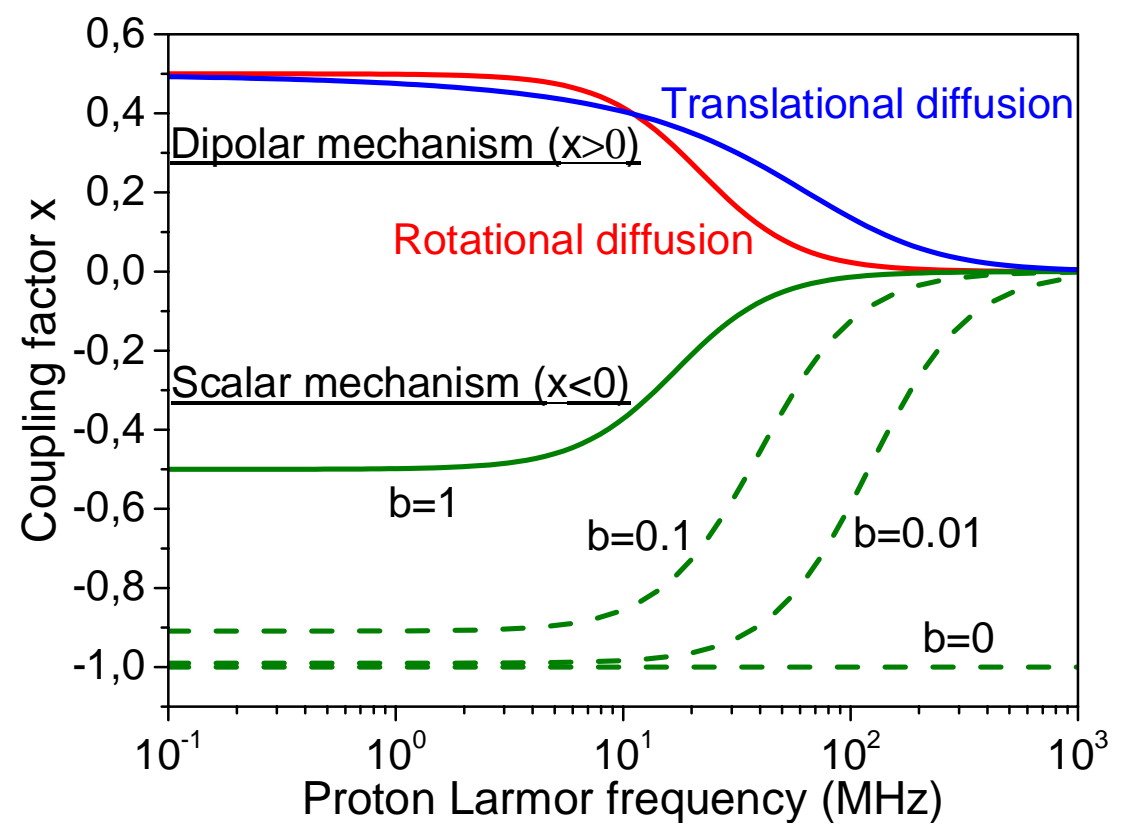

Figure 1.6: The coupling factor for different relaxation mechanisms (calculated for a correlation/diffusional time of $20 \mathrm{ps})^{76,77}$.

The dipolar and scalar contributions depend on the nature of an investigated system, nuclei and polarizers. The Overhauser DNP of protons is given by dipolar interaction in the most cases. The exceptions were found for aromatic protons and protons of trifluroacetic acid and reported by Müller-Warmuth ${ }^{75}$. The DNP experiments of ${ }^{13} \mathrm{C}$ demonstrated both dipolar (negative enhancement) and scalar (positive enhancement) contributions. Many works with such molecules as methanol, pyruvate, urea, acetone 


\section{Introduction}

showed the negative enhancement value ${ }^{78,79}$. A scalar coupling was observed in the hyperpolarization process of ${ }^{13} \mathrm{C}$ nuclei of some chlorinated carbons (for example, chloroform) ${ }^{75,78}$ and N,N-dimethylformamide ${ }^{79}$. Loening reported liquid-state DNP experiments at room temperature and $5 \mathrm{~T}$ of a magnetic field which demonstrated the scalar enhancement of ${ }^{31} \mathrm{P}$ (in triphenylphosphine), ${ }^{13} \mathrm{C}$ (in carbon tetrachloride), ${ }^{15} \mathrm{~N}$ (in aniline) and ${ }^{19} \mathrm{~F}$ (in hexafluorobenzene) using BDPA as polarizer ${ }^{66}$. The scalar contribution for ${ }^{19} \mathrm{~F}$ Overhauser DNP makes multi-frequency ${ }^{19} \mathrm{~F}$-NMR be a perspective to investigated fluorinated molecules used in the pharmaceutical and agrochemical context ${ }^{80}$. The field dependence of the dipolar coupling (Fig. 1.6) is a reason of the efficiency reduction of Overhauser mechanism at high magnetic field. Due to its field independence the scalar coupling opens the way of application of liquid-state Overhauser DNP at high magnetic field.

Temperature effects on the DNP efficiency through saturation and coupling factor by means of the broadening the EPR lines and changing correlation time, respectively. This dependence has been analysed in several works by different methods like nuclear magnetic relaxation dispersion (NMRD) measurement ${ }^{81}$, molecular dynamic simulation (MD) ${ }^{82}$ and the chemical shift change ${ }^{83}$ of water. The experimental measurement demonstrated a linear dependence of DNP enhancement on temperature that is in good agreements with predictions from MD simulation of the coupling factor (Fig. 1.7). 


\section{Introduction}

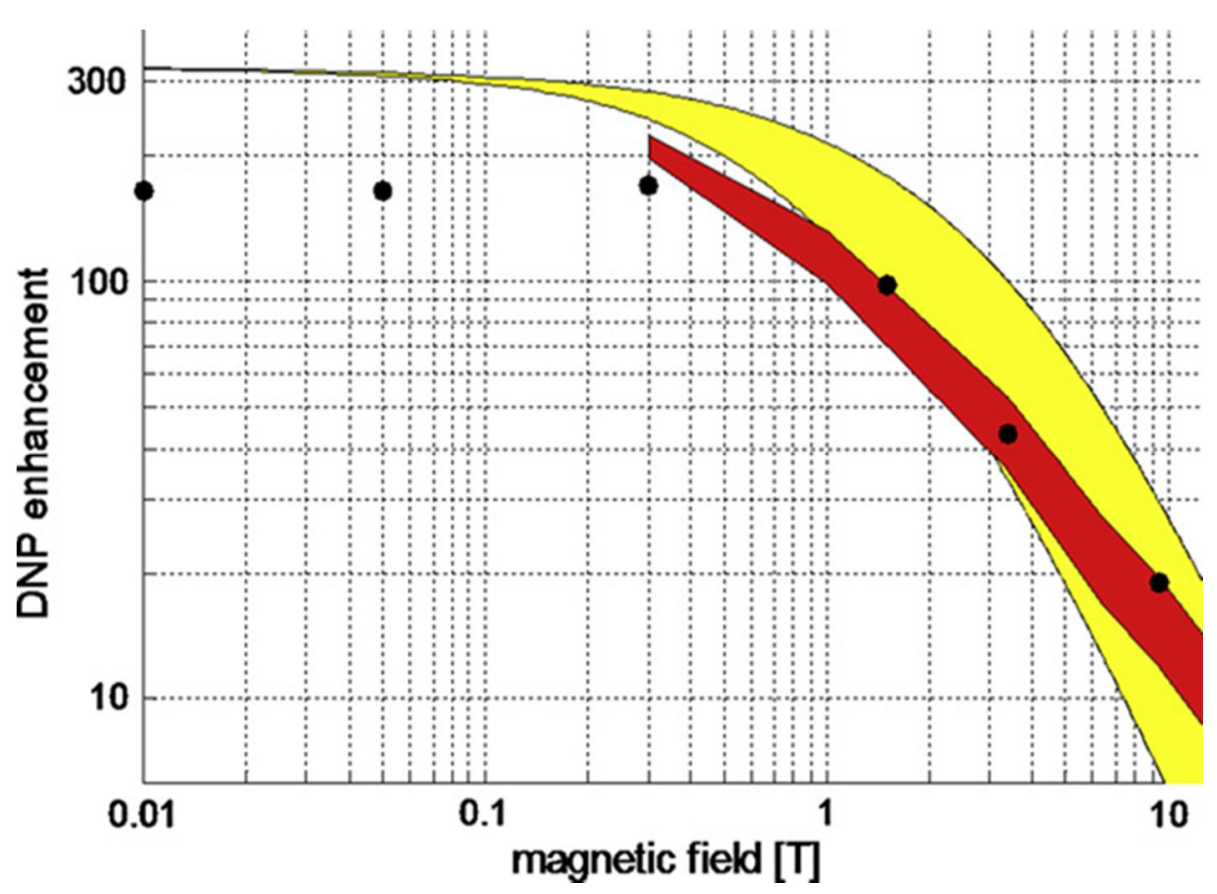

Figure 1.7: DNP enhancement of water protons produced by nitroxide radical at different magnetic field. Black cycles: experimentally observed values. The maximum values (assuming $s=1, f=1$ ) predicted from MD calculation for TEMPO in water (red) and from force-free model (yellow) are given for a temperature range from 25 to $45^{\circ} \mathrm{C}$. Adopted from ref. ${ }^{84}$.

1.2.3. Experimental approaches for liquid state DNP. Currently there are several different experimental setups for the application of DNP methods in NMR and MRI. The technical implementation can be classified as (Fig. 1.8):

- solution-state DNP, driven by the Overhauser mechanism in the liquids;

- solid-state DNP, driven by the cross effect at the low temperature in the solids;

- dissolution DNP, driven by the thermal mixing or the solid effect at low temperature and then sequential transfer of the fast dissolved sample to NMR/MRI magnet.

The solution-state DNP has two different ways of a realization. The first one proposes to create the hyperpolarization at low magnetic field and then transferred to the high field magnet for NMR detection. It is called shuttle DNP experiment. The second one deals with the hyperpolarization of the solution by Overhauser mechanism at a single high field strength. 


\section{Introduction}

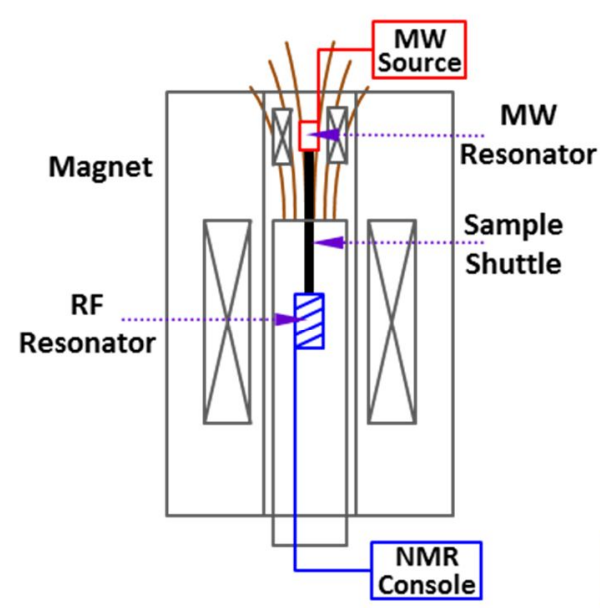

Shuttle DNP

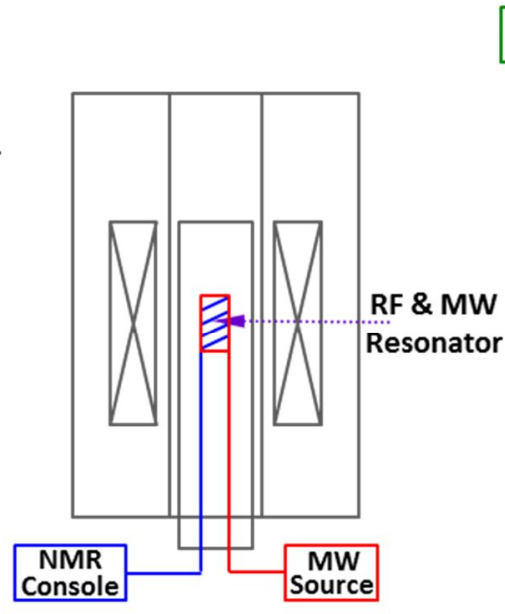

HF-liquid DNP

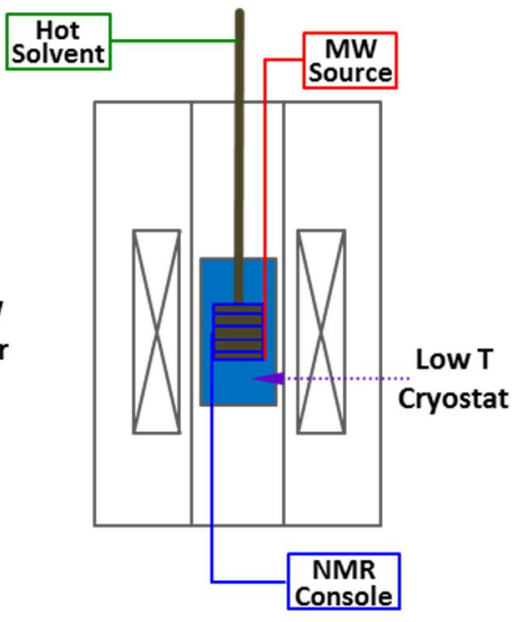

Dissolution DNP

Figure 1.8: Simple schemes of the experimental approaches for DNP experiments.

The first solution-state DNP experiment was done by Dorn et al. in $1988{ }^{85}$ where liquids continuously flowed in a $0.35 \mathrm{~T}$ magnet from the $\mathrm{MW}$ cell into RF cell $(9 \mathrm{GHz}$ and $14 \mathrm{MHz}$, respectively). Their second experiment was with two magnets where after an irradiation in a MW cavity $(0.35 \mathrm{~T})$ the sample flowed into NMR magnets $(4.7 \mathrm{~T}){ }^{86-88}$. The shuttle DNP concept, as alternative to continuous flow, is based on the rapid transfer hyperpolarized sample from one magnet to another. This principle was pioneered by Purcell and Pound in $1951^{89}$. Many results of shuttle DNP was reported by Bennati, Griesinger and cowokers ${ }^{78,90-92}$. They used a $0.35 \mathrm{~T}$ magnet as an EPR element $(9.7 \mathrm{GHz})$ placed on the top and a $14.09 \mathrm{~T}$ magnet as NMR $\left(600 \mathrm{MHz}{ }^{1} \mathrm{H}\right.$ Larmor frequency). In the last decade, the solution-state DNP at high magnetic field (up to $260 \mathrm{GHz}$ MW frequency), as one of important field of research, has been tackled by several groups like Prisner, Kentgens, Bennati and etc. ${ }^{66,93-100}$.

In 2003 Ardenkjaer-Larsen realized the dissolution DNP setup by designing the corresponding apparatus ${ }^{101}$. The sample was polarized at very low temperature (around $1 \mathrm{~K}$ ) and magnetic field $>1 \mathrm{~T}$ and then, after rapid dissolution by a hot solvent, transferred into a high field NMR magnet. The achieved DNP enhancement was larger than 10000. This principle has become the most popular for MRI application ${ }^{102-106}$.

Shuttle DNP. As it was described above the shuttle DNP spectrometer consists of an EPR spectrometer for polarization sample in result of the saturation of a polarizing agent 


\section{Introduction}

(radical) by MW irradiation and NMR spectrometer where then the sample is rapidly shuttled for signal detection ${ }^{89}$. The process can be repeated several times.

The DNP enhancement depends on the magnetic field jump between EPR and NMR magnets ${ }^{90}$ :

$$
\varepsilon\left(B_{N M R}\right)=\frac{I_{N M R}}{I_{0 N M R}\left(B_{N M R}\right)}=\frac{\varepsilon\left(B_{E P R}\right) \cdot I_{0 N M R}\left(B_{E P R}\right)}{I_{0 N M R}\left(B_{N M R}\right)}=\varepsilon\left(B_{E P R}\right) \cdot \frac{I_{0 N M R}\left(B_{E P R}\right)}{I_{0 N M R}\left(B_{N M R}\right)}=\varepsilon\left(B_{E P R}\right) \cdot \frac{B_{E P R}}{B_{N M R}}
$$

In the case of EPR field $0.35 \mathrm{~T}$ and NMR field $14 \mathrm{~T}$ the field jump reduces the enhancement by factor 40 . It means that the maximum possible enhancement in the case of pure dipolar mechanism is -8 instead -330 . The second problem is the loss of a hyperpolarization in result of the relaxation during sample shuttling from one magnet to other.

In 2009 Griesinger, Bennati and coworkers did the experiment using a first construction of a shuttle DNP spectrometer by combining EPR and NMR spectrometers with a shuttling tube (Fig. 1.9) ${ }^{78}$. This design of spectrometer has a disadvantage that a sample crosses zero field space between EPR and NMR magnets. 


\section{Introduction}
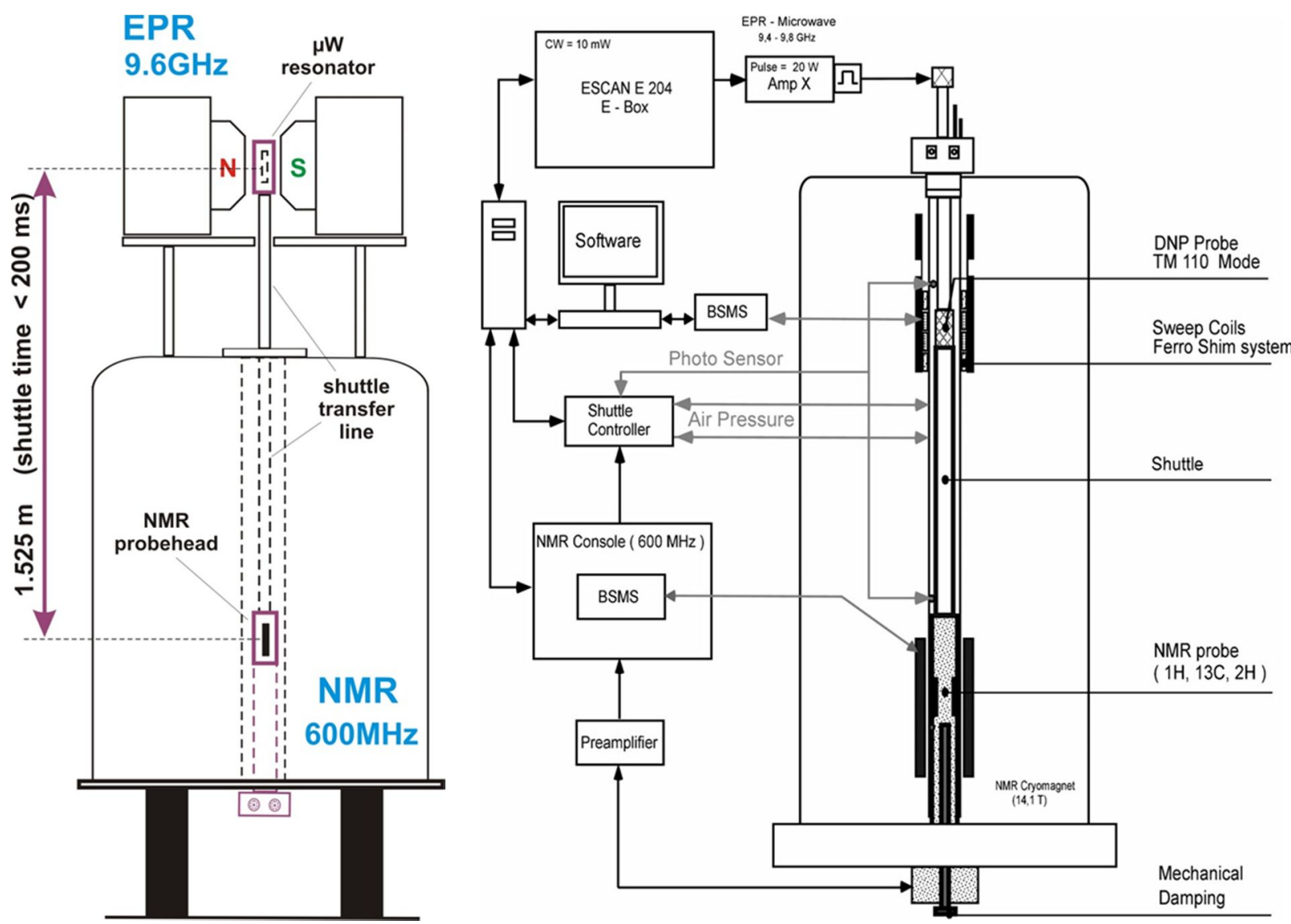

Figure 1.9: The schematic of the first shuttle DNP spectrometer (left) and the modified shuttle DNP spectrometer with two-centers magnet (right) ${ }^{78,91}$.

In 2010, a shuttle DNP spectrometer with two-center magnet was constructed by Bruker ${ }^{91}$ which consists of a $14 \mathrm{~T}(600 \mathrm{MHz}$ proton frequency) NMR cryomagnet magnet and a ferroshim system inserted in the stray field of that (Fig. 1.9). The ferroshim system provides a homogeneous low-field region (EPR) above the high-field magnetic center. The MW cavity with corresponding electron Larmor frequency is placed in that region. This modified construction allows minimizing relaxation-induced losses of hyperpolarization during the shuttling process. In contrast to the case of a complete absence of magnetic field between EPR and NMR parts in the prototype spectrometer, the field magnitude in the region of shuttling of the modified spectrometer never becomes less than $0.34 \mathrm{~T}$. Also the magnetic fields of EPR and NMR parts have the same directions unlike the prototype case. 


\section{Introduction}

Griesinger, Bennati and coworkers did several experiments using the shuttle DNP spectrometer with $0.34 \mathrm{~T}$ EPR and $14 \mathrm{~T}$ NMR and reported results ${ }^{78,90-92}$. The simple experiment with $25 \mathrm{mM}$ of TEMPONE- $\mathrm{D}_{16^{-}}{ }^{15} \mathrm{~N}$ in water solution showed the proton enhancement -2.6 . For $4 \mathrm{M}$ water solution of urea in presence of $25 \mathrm{mM}$ of the radical, the detected DNP enhancement of ${ }^{13} \mathrm{C}$ was $-4 \pm 1$. The achieved high field DNP enhancement of ${ }^{13} \mathrm{C}$ for chloroform was around +15 . Also in the case of experiment with urea it was showed that the low enhancement is a result of the counteraction of dipolar and scalar mechanism ${ }^{78}$. Using the shuttle spectrometer with the two-center magnet, a water proton enhancement with $5 \mathrm{mM}$ of TEMPONE- $\mathrm{D}_{16^{-}}{ }^{15} \mathrm{~N}$ can be achieved $-3.7{ }^{91}$. There were reported results of shuttle DNP experiment with D-glucose in $\mathrm{D}_{2} \mathrm{O}$ (with $10 \mathrm{mM}$ TEMPONE-D ${ }_{16^{-}}{ }^{15} \mathrm{~N}$ and $5 \mathrm{mM}$ DSS) where the different protons had enhancement in the range from -1.4 to $-2.8^{91}$. In 2012, doing the shuttle DNP experiment with L-tryptophan, they evaluated properties and reported several technical modifications which can improve stability and a reproducibility of the spectrometer ${ }^{92}$. The proton accessibility for the unpaired electrons is one of essential properties, which influences on the DNP enhancement ${ }^{92}$. The shuttle DNP with a two-center magnet, which partially has a short shuttle distance, can have perspectives for application to study biomolecules.

High-field liquid-state DNP. The first DNP spectrometer for liquid-state experiments operating at a single high field strength (at $9.2 \mathrm{~T}$, around $300 \mathrm{MHz}$ of proton frequency) for EPR and NMR was designed in Frankfurt ${ }^{96}$. Two main problems of Overhauser DNP experiment at high magnetic field are a heating effect and a reduction of the coupling factor in the case of a dipolar contribution (Fig. 1.6).

Bennati et al. examined experimentally the field dependence of the characteristic factors of Overhauser mechanism at $9.7 \mathrm{GHz}$ and $94 \mathrm{GHz}$, using TEMPONE-D ${ }_{16^{-}}{ }^{15} \mathrm{~N}$ as polarizer in water solution ${ }^{81,93}$. The reached DNP enhancement of water protons values were -170 at $9.7 \mathrm{GHz}$ and -43 at $94 \mathrm{GHz}{ }^{93}$. The systematically study of the saturation factor demonstrated that its dependence on magnetic field is negligible in contrast to MW power. The coupling factor has values around 0.36 at $5-10 \mathrm{mM}$ and drop to 0.33 at 25-50 mM. It is in good agreement with NMRD measurements ${ }^{81}$ and MD estimations ${ }^{82}$. 


\section{Introduction}

Using a modified Bruker ENDOR probe for experiments with TEMPOL in toluene at $94 \mathrm{GHz}$ Kryukov et al. observed the enhancements -50 and -40 for ring and methyl protons, respectively ${ }^{100}$. These values are significantly larger than predicted by an extrapolation of low-field experiments.

Due to the MW attenuation in a dielectric lossy medium (for example, water) the decreasing of a sample volume is required which is estimated according to the characteristic penetration depth $(d)^{70}$

$$
\left(\frac{1}{d}\right)^{2}=\frac{1}{2} \varepsilon_{0} \mu_{0} \cdot(2 \pi v)^{2} \cdot\left(\left|\varepsilon_{r}\right|-\varepsilon_{r}^{\prime}\right),
$$

where $\varepsilon_{0}$ and $\mu_{0}$ are the electrical permittivity and the magnetic permeability in vacuum, respectively, $\varepsilon=\varepsilon_{r}{ }^{\prime}+i \cdot \varepsilon_{r}{ }^{\prime \prime}$ ( $i$ is the imaginary unit) is the relative permittivity of the medium, $v$ is MW frequency. Therefore, the sample volume for high field experiments scales with $1 / v^{3}$. The penetration depths of MW in water are $\sim 2.8 \mathrm{~mm}$ and $\sim 0.24 \mathrm{~mm}$ for $9 \mathrm{GHz}$ and $94 \mathrm{GHz}$, respectively. To minimize heating effect the separation of electrical and magnetic components at sample position is required in a multi-frequency resonator for $\mathrm{DNP}^{70}$.

Prisner and coworkers developed the high-field DNP spectrometer with a helix double-resonance structure for liquid-state experiments ${ }^{96}$. That structure allows avoiding high heating effect typical for aqueous solution. Later, this resonator was included in their high field implementation for liquid-state DNP at 9.2 T (400 MHz NMR, $260 \mathrm{GHz}$ of MW source) where a high power gyrotron was used as MW source ${ }^{97}$. However, this spectrometer construction has very poor radiofrequency homogeneity and requires tiny capillary samples for experiments.

In 2012, Denysenkov and Prisner presented the probe with a Fabry-Perot resonator for DNP at 9.2 T which allows using a 30-fold larger sample volume of aqueous solution than in previous works ${ }^{107}$. Used the plain mirror of the semiconfocal Fabry-Perot resonator as NMR coil allows to place and distribute the sample on the surface of that mirror to reach a necessary small depth for avoiding heating effect. Jakdetchai et al. 


\section{Introduction}

demonstrated a significant Overhauser DNP on aligned lipid bilayers at room temperature using the Fabry-Perot resonator ${ }^{108}$. A monoradical TEMPO and biradicals bTbK and TOTAPOL were used as polarizing agents. Partially, they showed that the efficient polarization transfer governed by Overhauser effect at high field can be realized within insoluble samples.

It was found that Fremy's salt as a polarizer, exhibiting a narrow EPR linewidth even at $260 \mathrm{GHz}(<0.1 \mathrm{mT})$, yields higher DNP enhancement than TEMPO radicals 95,99 . Using gyrotron MW source DNP experiment with Fremy's salt gave an enhancement of water protons around -30 which is 3 times larger than that one in the case of $45 \mathrm{~mW}$ source ${ }^{97}$.

As it was above described the scalar contribution to the coupling factor does not depends on magnetic field. Unlike proton ${ }^{1} \mathrm{H}$, the interaction between ${ }^{13} \mathrm{C}$ and an unpaired electron of a radical has the scalar compound that allows overcoming several typical problems for ${ }^{1} \mathrm{H}$ in high-field DNP. It was demonstrated by Loening and coworkers ${ }^{66,80}$.

Dissolution DNP. Dissolution DNP (ex situ DNP) was pioneered by ArdenkjaerLarsen in 2003. At first a sample was placed in a DNP setup, which consists of a superconducting magnet $3.4 \mathrm{~T}(\mathrm{MW} 94 \mathrm{GHz})$ and low temperature cryostat with liquid helium, and irradiated by MW at $1.1 \mathrm{~K}$. After a quick dissolution process by a hot solvent injection, the polarized sample was rapidly transferred into a high-resolution 9.4 T NMR spectrometer for signal ${ }^{13} \mathrm{C}$-detection ${ }^{101}$. The result hyperpolarized sample can be used as a contrast agent for in vivo MRI experiment.

The dissolution DNP has a sharp leap of temperature. Including the temperature dependence of the polarization (1.2), the overall enhancement in the dissolution DNP experiment is defined by:

$$
\varepsilon\left(B_{N M R}, T_{N M R}\right)=\varepsilon\left(B_{E P R}, T_{E P R}\right) \cdot \frac{B_{E P R} / T_{E P R}}{B_{N M R} / T_{N M R}}=\varepsilon\left(B_{E P R}, T_{E P R}\right) \cdot \frac{B_{E P R}}{B_{N M R}} \cdot \frac{T_{N M R}}{T_{E P R}}
$$




\section{Introduction}

In the case of a $9.4 \mathrm{~T} \mathrm{NMR}$ at room temperature the magnetic field ratio is $\approx 0.36$ whereas the temperature one is $\approx 270$. The enhancement prefactor is $\approx 100$ so, if $\varepsilon\left(B_{E P R}, T_{E P R}\right) \approx 120$, the overall DNP enhancement should be $>10000$.

The design of the dissolution DNP spectrometer requires the EPR magnet with liquidhelium cryogen for very low temperature, MW source with quasioptical tube for MW transmission and detection, a tube for a sample transfer into NMR or MRI spectrometers, heating compound for quickly dissolution of a sample to ambient conditions before NMR detection ${ }^{101,109,110}$. Ernst et al. designed an additional useful element "revolver" for multi-sample experiments ${ }^{111}$. In 2010 Köckenberger et al. developed a dedicated design spectrometer using a magnet with two isoceneters and shuttle actuator between them. The top compartment of this magnet is for DNP generation at $3.4 \mathrm{~T}$ and low temperature whereas the bottom one is for NMR detection at $9.4 \mathrm{~T}$ in liquid state ${ }^{112}$. One important problem of the existing construction of the dissolution DNP spectrometer is the irreversible melting/dissolution of the sample.

The samples for the dissolution DNP are usually prepared in a glassy matrix using solvents like water, methanol, glycerol. The dissolution process is realized by means of hot pressurized solvents (at $200{ }^{\circ} \mathrm{C}$ and 10 bar). The sample transfer into a NMR compartment is achieved by gas pressure. The additional methanol compound is used often to reduce water bubbles appearing during the transfer process. To reduce the overall time ( $\sim 3 \mathrm{~s}$ ) of the dissolution and the sample transfer processes Hilty et al. developed the special sample injection device thus the taken time became around $600 \mathrm{~ms}$. This improved reconstruction allowed to detect high quality spectra of glucose ${ }^{113}$.

The presence of the radical in the system has two negative effects. The first, general, is the increased relaxation rate of the polarized sample. The second is the toxicity for in vivo applications. Ardenkaer-Larsen et al. used several methods to remove radicals, such as a filtration through a short anion-exchange column ${ }^{101}$ and a precipitation under acidic condition ${ }^{109}$. Zweier et al. solved this problem by the method of a radical quenching using a reducing agent ${ }^{114}$. 


\section{Introduction}

When Ardenkaer-Larsen and coworkers did the dissolution DNP experiment with ${ }^{13} \mathrm{C}$-urea in glycerol using a trityl radical as polarizer it was observed according to microwave sweeps (polarization vs. MW frequency) and build-up curves that the thermal mixing (TM) mechanism was dominated ${ }^{101}$. Working with various nuclei, as ${ }^{2} \mathrm{H},{ }^{13} \mathrm{C}$, ${ }^{15} \mathrm{~N},{ }^{31} \mathrm{P},{ }^{29} \mathrm{Si}$, Reynolds determined the polarization mechanisms. According to the measurements of the frequency separation of cross transitions and the nuclear Larmor frequency (Fig. 1.4) he found that ${ }^{31} \mathrm{P}$ nucleus was polarized through a solid effect (SE) whereas the thermal mixing (TM) was for other three nuclei $\left({ }^{2} \mathrm{H},{ }^{13} \mathrm{C},{ }^{15} \mathrm{~N},{ }^{29} \mathrm{Si}\right){ }^{115}$.

To overcome the problem of a fast nuclear relaxation the idea of long-lived tags was used in several experiments. Wilson et al. decide to attach acetyl tags (because of its long relaxation time) to other molecules near $\mathrm{OH}$ or $\mathrm{NH}_{2}$ groups ${ }^{116}$. He used this tag to resolve spectra of some amino acids in various peptides. Another way was considered by Bodenhausen et al. exploring a long-lived singlet spin state ${ }^{117}$. As shown by Levitt and coworkers, long-lived singlet states are delocalized on two or more coupled spins and have unusually long relaxation times because they are not affected by intramolecular dipole-dipole relaxation ${ }^{118-120}$.

Dissolution DNP becomes rapidly successful for MRI in vivo and medical diagnostics 102, 103, 121 . Currently it is actively used for detection of behavior of ${ }^{13} \mathrm{C}(1)$-pyruvate in tumors ${ }^{105}$ and for measurement $\mathrm{pH}$ using ${ }^{13} \mathrm{C}$-labelled bicarbonate ${ }^{122,123}$. The dissolution NMR is perspective for an analysis of biochemical reactions, enzymes and molecular ${ }^{124-}$ 128

1.2.4. Current applications of liquid-state DNP. Overhauser dynamic nuclear polarization is a perspective tool to study hydration dynamic ${ }^{129-134}$. Han and coworkers studied the dynamic of water at lipid vesicle surface ${ }^{135}$ and site-specific hydration translational dynamics in the nonpolar core of a molten globule ${ }^{132}$. After the quantitative analysis they classified the empirical hydration dynamics into several regimes of the coupling factor which characterizes the nature of the interaction between investigated biological system and the nearby hydration water ${ }^{136}$ (Fig. 1.10). 


\section{Introduction}

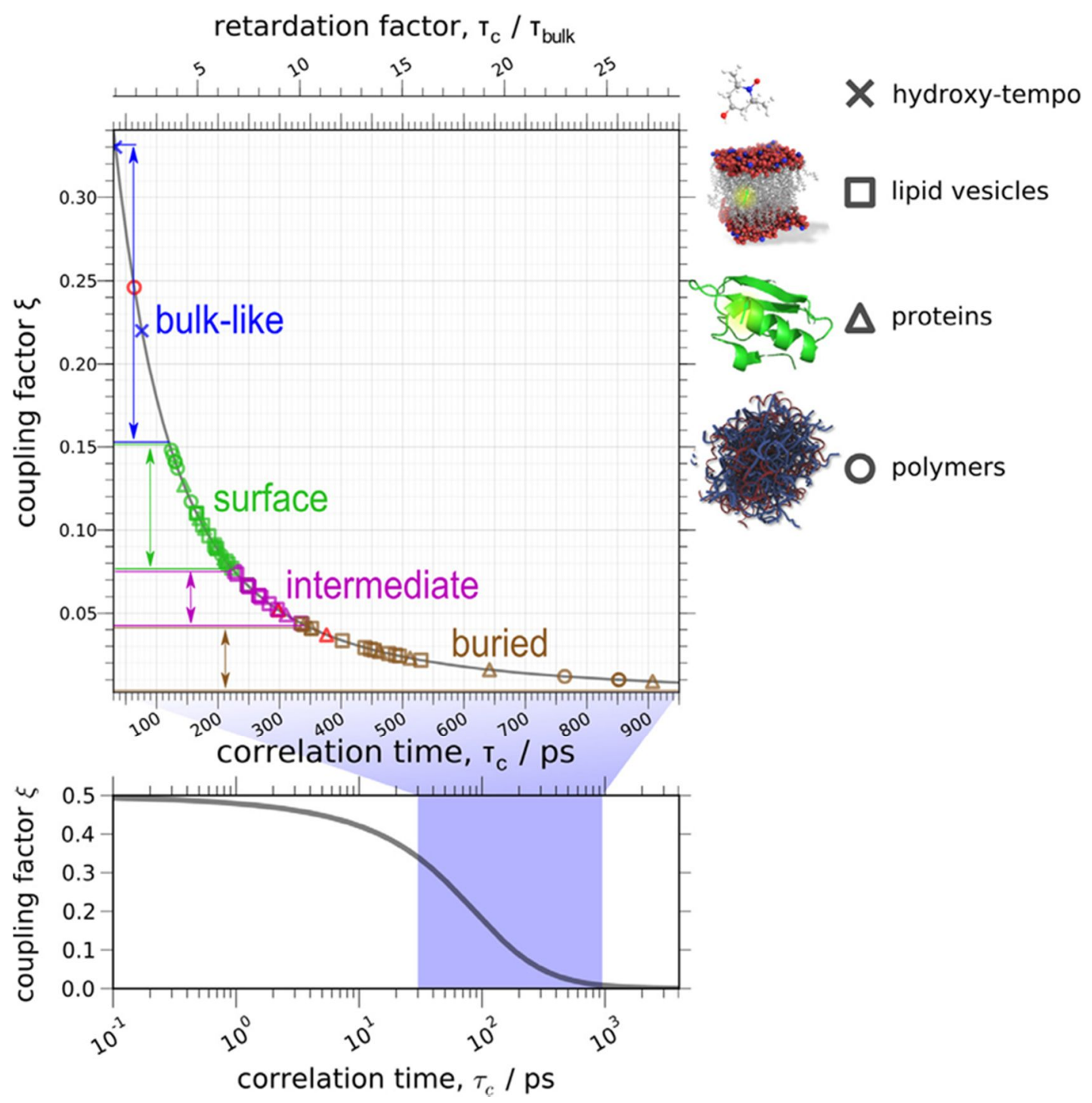

Figure 1.10: The dependence of the coupling factor $\xi$ on the correlation time $\tau_{c}$. Right: the systems correspondent to the shown regimes of classification. From ref. ${ }^{136}$.

Armstrong and Han did DNP experiments at $0.35 \mathrm{~T}$ with site-specifically spin-labelled proteins ${ }^{132}$. The proton-NMR signal of water carries information about the local dynamic. The study with molten globule (MG) demonstrated that all the MG core sites directly interact with water bearing significant translational dynamics. It provided a highresolution glimpse at the folding-dependent nature of protein hydration.

Doll and Jeschke demonstrated the liquid-state DNP as the method for an extraction of qualitative site-specific water accessibility on spin-labelled membrane proteins ${ }^{137}$. This study provides very important information about protein structure and function at physiological condition. The vitamin $\mathrm{B}_{12}$ importer BtuCD was investigated in two states 


\section{Introduction}

(detergent-solubilized and reconstituted). It was found that the DNP is very sensitive for water accessibility analysis in spite of low concentration of the investigated sample $(<100 \mu \mathrm{M})$.

Valentine et al. used reverse micelles as an implement for liquid-state DNP with proteins ${ }^{138}$. In contrast to aqueous samples, the low conductivity of reverse micelle solution allows to avoid significant heating. Using three case of the nitroxide radical introduction into the reverse micelle system (Fig. 1.11) the highest DNP enhancement $(\approx-93)$ was observed when the radical is solubilized in the reverse micelle surfactant shell. The hydration properties of encapsulated proteins can improve the polarization transfer efficiency from water to the protein. The obtained results demonstrate the reverse micelle encapsulation as a perspective technology to improve liquid-state DNP application for NMR analysis of proteins and other biomolecules.
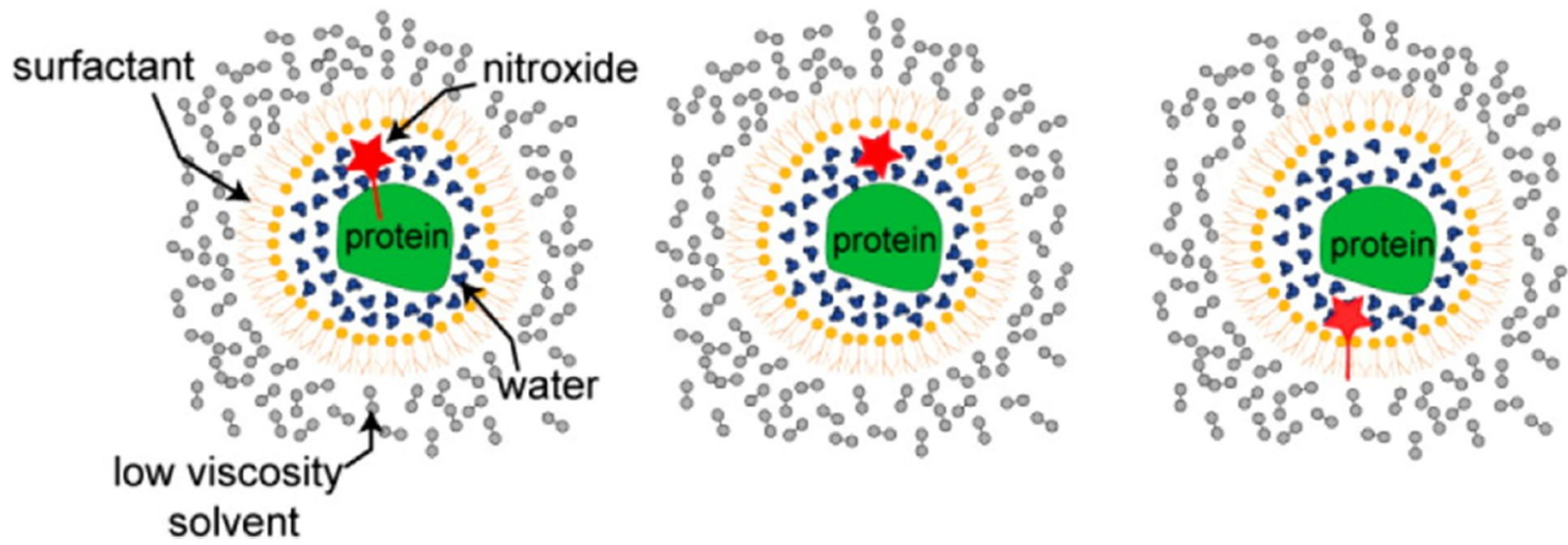

Figure 1.11: Illustrations of the introduction of radicals into reverse micelles. Left: covalently linked to the protein. Middle: dissolved in the aqueous core. Right: attached to a carrier in the surfactant shell. From ref. ${ }^{138}$

1.2.5. Polarizing agents for DNP. The polarizing agent is a main component of the DNP experiment. Radicals are common polarizing agents used for the above described DNP mechanisms (Fig. 1.12). The efficiency of DNP strongly depends on properties of radicals like relaxation times of unpaired electrons, solubility in a suitable solvent (water in the case of biological applications), spin relaxations and etc. 


\section{Introduction}
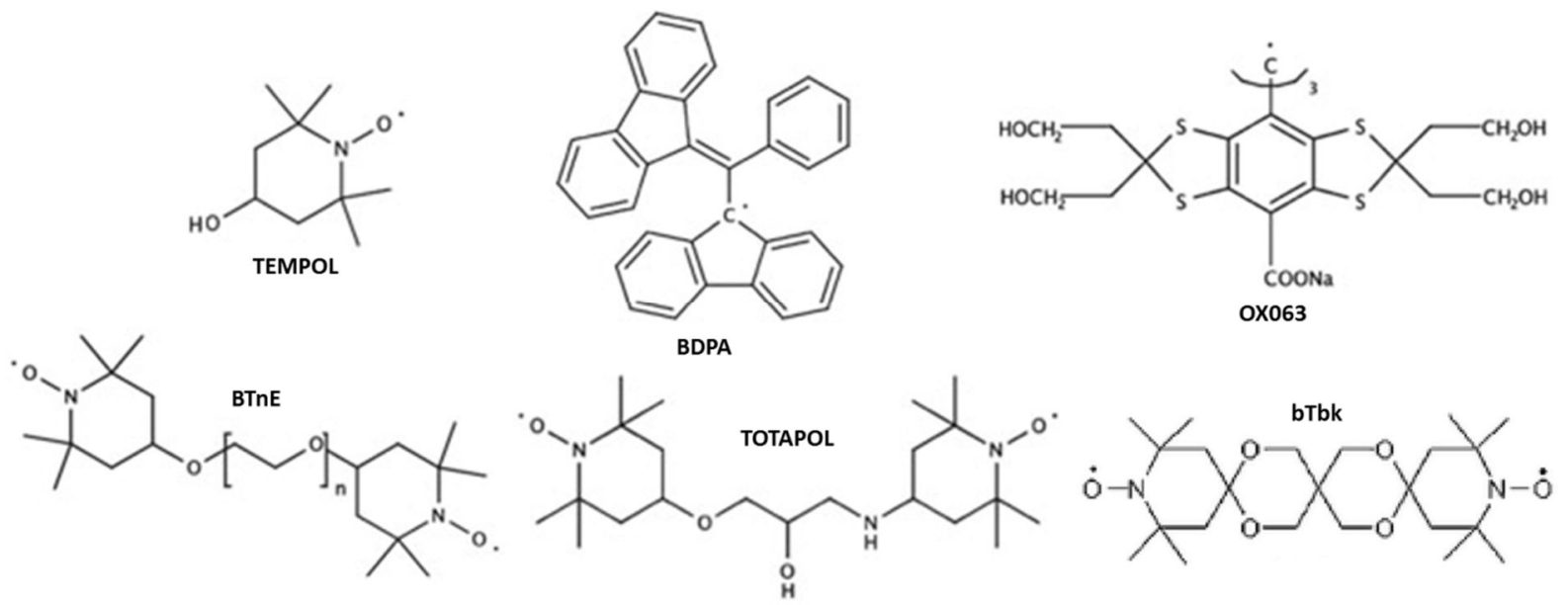

Figure 1.12: The most popular stable radicals/biradicals used in DNP experiments.

Nitroxide radicals (TEMPO, 4-hydroxy-TEMPO, 4-oxo-TEMPO) are the most commonly used for Overhauser DNP in liquids. As it was shown TEMPO radical with ${ }^{15} \mathrm{~N}$ and ${ }^{2} \mathrm{H}$ have higher saturation at the same conditions than standard ones ${ }^{72}$. TEMPObased biradicals are applied for solid-state DNP governed by the cross effect (CE). Griffin and coworkers developed several biradicals (BTnE, TOTAPOL, bTbk) which later became widespread ${ }^{139-141}$.

The solid effect (SE) mechanism requires polarizers with narrow EPR lines. This requirement can be satisfied for radicals with a symmetry property. Trityl radicals fit it well and OX063 radical (Fig. 1.12) is from this kind of polarizer which is commonly used in dissolution DNP (where SE is dominant mechanisms of polarization in frozen solutions) ${ }^{101,142}$.

A series of research works introduced DNP experiments where spin-labelled (usually by TEMPO radicals) polymers were used as polarizers ${ }^{143-145}$. These kinds of polarizing systems demonstrated specific advantages which solve typical problems with small free radicals like small saturation, fast spin relaxations, difficulty of filtration.

In 2011 Corzilius demonstrated the possibility to use transition metal complexes $\left(\mathrm{Gd}^{3+}\right.$ and $\mathrm{Mn}^{2+}$ ) as polarizing agents for DNP experiments ${ }^{146}$. The availability of a narrow EPR line of the metal complex with $\mathrm{Gd}^{3+}$ makes the last one be comparable with trityl radical as polarizer. 


\section{Introduction}

\subsection{Dynamic electron polarization (DEP) for DNP with photoexcited electron state}

Dynamic electron polarization (DEP) is generating a nonequilibrium electron spin polarization. Two kinds of spin hyperpolarization have been developed by optical pumping. One of them is based on generation the excited triplet state which has a nonequilibrium spin population distribution due to spin-level-selective intersystem crossing transition from an excited singlet state ${ }^{147-149}$. The second one is realised by a circular polarized light irradiation, which leads to selective transitions from ground to excited state ${ }^{18}$. Chemically induced dynamic electron polarization (CIDEP) is usually due to interaction of the excited triplet state of a dye with a radical ${ }^{150-155}$.

The Overhauser DNP enhancement with the radical in the thermal equilibrium state is limited by value of the ratio $\gamma_{e} / \gamma_{I}\left(660\right.$ for $\left.{ }^{1} \mathrm{H}\right)$. This limit can be overcome using the photoexcited triplet state, which polarization is ordinary very high at ambient conditions (far from thermal equilibrium polarization) and it does not depend on external magnetic field. In result of a chemical reaction of the excited triplet state with a suitable substrate, where a rate is rapid enough to compete a spin lattice relaxation inside the triplet state.

Henstra et al. presented the DNP experiment on a single crystal of naphthalene with pentacene dopant inside that crystal ${ }^{44}$. The hyperpolarization of photoexcited triplet state of pentacene (by a pulsed nitrogen laser) was transferred to protons of naphthalene due to the integrated solid effect (ISE). The reached enhancement was of 5500. Takeda et al. extended this method of nuclear polarization for case a polycrystalline sample using the same system of pentacene-doped naphthalene ${ }^{63}$. The achieved value of the proton enhancement was 3160. Tateishi and coworkers observed that hyperpolarization of ptotoexcited pentacene can also be transferred to nuclei of glassy matrix which it is doped into ${ }^{156,157}$. The hyperpolarization diffuses from the nuclear spins in the vicinity of pentacene to the rest of the sample. Also the nuclei of molecules codoped with pentacene in the glassy matrix can be hyperpolarized. I was found for ${ }^{19} \mathrm{~F}$ nuclei of 2,3,4trifluorobenzoic acid and 5-fluorouracil ${ }^{156}$. 


\section{Introduction}

The interaction of a free radical with a photoexcited triplet state leads to a hyperpolarization of the radical. The effect is based on the transfer of a non-equilibrium spin polarization from the triplet state, which is formed by intersystem crossing (ISC), to the ground state of the radical ${ }^{147,158}$. Corvaja et al. investigated several covalently linked fullerene-nitroxide systems ${ }^{154,159-162}$. The ground and quartet states were detected by CW transient EPR. The latter state is a result of the superposition of doublet with triplet. They described the reversed quartet mechanism which explains the inversion polarization sign in a microsecond time scale ${ }^{154}$.

\subsection{The aims of this work}

The hypothesis consists in generating of non-equilibrium electron polarization of a radical by continuous light irradiation of a covalently linked dye and a successive spin polarization transfer to target nuclei of solvents and/or molecules of interest (Fig. 1.13). Perspectives of this hypothetic method are the very high initial electron spin polarization, which can be transferred to the target nuclei (overcoming the maximum Overhauser DNP enhancement value of 660), and the absence of MW irradiation. The latter allow overcoming the volume issue concerned with a heating effect.

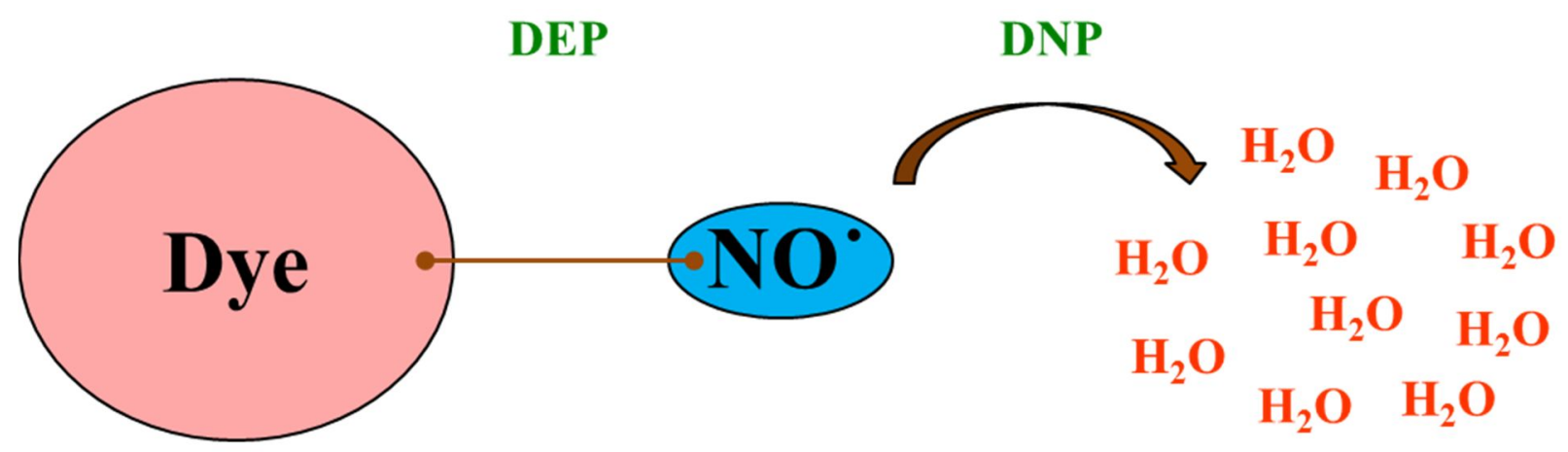

Figure 1.13: Possible strategy of spin polarization transfer from the photoexcited triplet state to nuclei. 


\section{Introduction}

The aims of this research work are:

- to analyse DNP with nitroxide radical in toluene, which is a perspective non-polar solvent to host many complex organic polarizers which are water-insoluble, and compare the experimental result with published theoretical molecular dynamic investigations;

- to synthesize fullerene-nitroxide derivatives to improve water solubility and carry out DNP experiments with them;

- to analyse solvent and polarizer's size effects in liquid-state DNP;

- to describe relaxation effects between EPR hyperfine lines and obtain analytical expressions of the polarization recovery and saturation factors of each line;

- to demonstrate experimentally the independency of the effective saturation factor from polarizer concentration in case of dominant nitrogen nuclear relaxation resulting in constant DNP efficiency;

- to estimate possible perspectives of photoexcited fullerene-nitroxide derivatives as an alternative way of DNP without MW. 


\section{Theoretical background}

\subsection{Overhauser mechanism}

According to principles of quantum mechanics the Hamiltonian of an electron-nuclear coupled system in DNP experiment is given by

$$
\begin{gathered}
H=H_{E B}+H_{N B}+H_{E N} \\
H_{E B}=\omega_{S} \cdot S_{z}, H_{N B}=-\omega_{I} \cdot I_{z}, H_{E N}=H_{E N}^{D}+H_{E N}^{S c},
\end{gathered}
$$

where $H_{E B}$ and $H_{N B}$ are the Hamiltonian for electron and nuclear Zeeman interaction, respectively; $H_{E N}$ is Hamiltonian of electron-nuclear interaction which consists of dipolar $H_{E N}^{D}$ and scalar (Fermi contact) $H_{E N}^{S c}$ interactions. The term of dipolar electron-nuclear interaction is expressed as

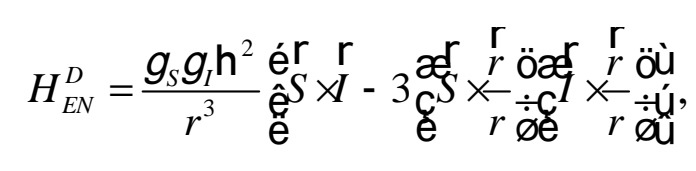

where $\dot{r}$ is the distance vector between the electron and nucleus; $\gamma_{S}$ and $\gamma_{I}$ are electron and nuclear gyromagnetic ratios, respectively. The term of scalar interaction can be written as

$$
H_{E B}^{S c}=a \cdot \stackrel{\prime}{I} \cdot \stackrel{\prime}{S}
$$

where $a$ represents the scalar hyperfine coupling strength.

In liquids, DNP is governed by the Overhauser effect ${ }^{7}$. It transfers the thermal equilibrium polarization of electron spins to coupled nuclei by microwave irradiation inducing the electron spin transition. Later this phenomenon was explained by the existence of cross transitions in the electron-nuclear coupled system (Fig. 2.1 $)^{7,69}$. 


\section{Theoretical background}

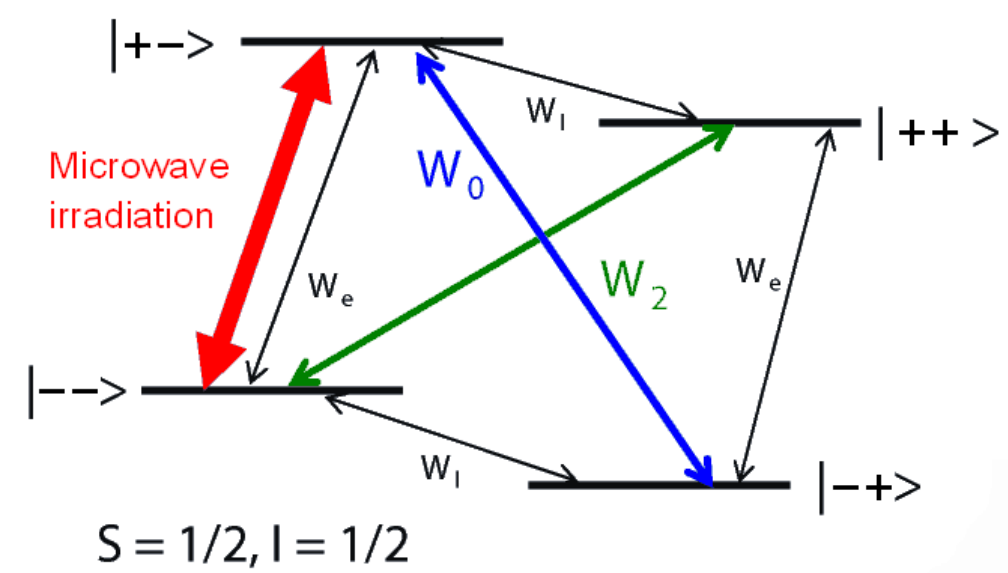

Figure 2.1: Energy levels and pathways of the electron-proton coupled spin system.

The population redistribution of the spin states after MW irradiation can be described by the following rate equation $\left.\left.\left.\left.\left(N_{1} Æ E I-+\right\rangle, N_{2} Æ E I--\right\rangle, N_{3} Æ E I++\right\rangle, N_{4} Æ E I+-\right\rangle\right)$ :

$$
\frac{d}{d t}\left(\begin{array}{l}
N_{1} \\
N_{2} \\
N_{3} \\
N_{4}
\end{array}\right)=\left(\begin{array}{cccc}
-w_{e}-w_{I}-w_{0} & w_{I} & w_{e} & w_{0} \\
w_{I} & -w_{e}-w_{I}-w_{2} & w_{2} & w_{I} \\
w_{e} & w_{2} & -w_{e}-w_{I}-w_{2} & w_{I} \\
w_{0} & w_{e} & w_{I} & -w_{e}-w_{I}-w_{0}
\end{array}\right)\left(\begin{array}{l}
N_{1}-N_{1}^{B} \\
N_{2}-N_{2}^{B} \\
N_{3}-N_{3}^{B} \\
N_{4}-N_{4}^{B}
\end{array}\right),
$$

where $w_{e}$ and $w_{I}$ are probabilities of electron and nuclear transitions, respectively; $w_{0}$ and $w_{2}$ are probabilities of forbidden cross transitions (called zero and double quantum transitions), which flip both electron and nuclear spins simultaneously and play a key role in Overhauser effect. $N_{i}^{B}(i=1, \ldots, 4)$ correspond to the Boltzmann level populations. The zero quantum transition is induced by both dipolar and scalar interactions between an electron and a nucleus whereas the double quantum transition depends only on scalar interaction.

The total EPR and NMR magnetisation values $\left(S_{z}, I_{z}\right)$ are expressed in form of the corresponding population differences: $\left\langle S_{z}\right\rangle \sim\left(N_{1}-N_{2}\right)+\left(N_{3}-N_{4}\right),\left\langle I_{z}\right\rangle \sim\left(N_{1}-N_{3}\right)+\left(N_{2}-N_{4}\right)$. The total population is constant: $N=N_{1}+N_{2}+N_{3}+N_{4}+N_{5}+N_{6}=N^{B}$. To perform a linear equation transformation, an additional parameter $\Delta n=N_{1}-N_{2}-N_{3}+N_{4}$ is included. The new linear equation is: 


\section{Theoretical background}

$$
\frac{d}{d t}\left(\begin{array}{c}
N \\
\left\langle S_{z}\right\rangle \\
\left\langle I_{z}\right\rangle \\
\Delta n
\end{array}\right)=\left(\begin{array}{cccc}
0 & 0 & 0 & 0 \\
0 & -2 w_{e}-w_{0}-w_{2} & w_{2}-w_{0} & 0 \\
0 & w_{2}-w_{0} & -2 w_{I}-w_{0}-w_{2} & 0 \\
0 & 0 & 0 & -2 w_{e}-2 w_{I}
\end{array}\right)\left(\begin{array}{c}
N \\
\left\langle S_{z}\right\rangle-S_{z}^{B} \\
\left\langle I_{z}\right\rangle-I_{z}^{B} \\
\Delta n-\Delta n^{B}
\end{array}\right)
$$

The total relaxation rate of nuclear spin $T_{1 I}^{-1}\left(T_{1 I}\right.$ is spin-lattice relaxation time) consists of the relaxation in the absence of electronic spins $\left(R_{I 0}\right)$ and the relaxation caused by dynamics of the considered coupled system (paramagnetic relaxation, $\left.R_{I \text { para }}\right)^{7}$ :

$$
1 / T_{1 I}=R_{I 0}+2 w_{I}+w_{0}+w_{2}=R_{I 0}+R_{I \text { para }} .
$$

The microwave (MW) pumping of the electron spin resonance line (Fig. 2.1), which leads to its saturation, the spin-lattice relaxations and the cross transitions drive the electron-nuclear coupled system to the steady state. The steady state solution for $\left\langle I_{z}\right\rangle$ from equation (2.5) is following:

$$
\begin{aligned}
& \frac{d}{d t}\left\langle I_{z}\right\rangle=-\left(R_{I 0}+2 w_{I}+w_{0}+w_{2}\right)\left(\left\langle I_{z}\right\rangle-I_{z}^{B}\right)+\left(w_{2}-w_{0}\right)\left(\left\langle S_{z}\right\rangle-S_{z}^{B}\right)=0 \\
& \left\langle I_{z}\right\rangle=I_{z}^{B}\left(1+\frac{w_{2}-w_{0}}{R_{I 0}+2 w_{I}+w_{0}+w_{2}} \cdot \frac{\left\langle S_{z}\right\rangle-S_{z}^{B}}{S_{z}^{B}} \cdot \frac{S_{z}^{B}}{I_{z}^{B}}\right)
\end{aligned}
$$

The following parameters $\xi, f, s$, which are called the coupling, leakage and the saturation factors, respectively ${ }^{7}$, will be used to describe the Overhauser mechanism:

$$
\xi=\frac{w_{2}-w_{0}}{2 w_{I}+w_{0}+w_{2}}, \quad f=\frac{2 w_{I}+w_{0}+w_{2}}{2 w_{I}+w_{0}+w_{2}+R_{I 0}}, \quad s=\frac{S_{z}^{B}-\left\langle S_{z}\right\rangle}{S_{z}^{B}}
$$

Since the thermal equilibrium magnetization at low magnetic field is proportional to the gyromagnetic ratio, the factor $\left(S_{z}^{B} / I_{z}^{B}\right)$ can be replaced by the quotient of the electron and nuclear gyromagnetic ratios $\left(\gamma_{S} / \gamma_{I}\right)$. Therefore, DNP enhancement $\left(\varepsilon=\left\langle I_{z}\right\rangle / I_{z}^{B}\right)$ of an NMR signal is described by the following Overhauser equation (2.7) ${ }^{7}$ :

$$
\varepsilon=1-\xi \cdot f \cdot s \cdot \frac{\left|\gamma_{S}\right|}{\gamma_{I}}
$$




\section{Theoretical background}

The time-dependent Overhauser equation, describing the build-up of the nuclear enhancement with the duration of a MW irradiation, $\tau \gg T_{1 e}\left(\left\langle S_{z}\right\rangle \approx\right.$ const $)$, is following:

$$
\varepsilon(\tau)=1-\xi \cdot f \cdot s \cdot \frac{\left|\gamma_{S}\right|}{\gamma_{I}}\left(1-e^{-\tau / T_{1 I}}\right)
$$

2.1.1. Saturation factor of EPR lines. The saturation factor $s$ (2.8) describes to what extend the microwave irradiation saturates an EPR line. Complete saturation means $\left\langle S_{z}\right\rangle=0$ the equal population of the Zeeman spin levels. In more general case, when electron polarization deviates from thermal equilibrium, this factor is related to the relative electron polarization $\left(\varepsilon_{e}\right)$ :

$$
s=\frac{S_{z}^{B}-\left\langle S_{z}\right\rangle}{S_{z}^{B}}=1-\varepsilon_{e} .
$$

The maximum for a DNP experiment is the complete saturation $(s=1)$ of an irradiated EPR line, as asymptotic value at infinite MW power $\left(P_{M W} \rightarrow \infty\right)$. The power dependence of the CW EPR signal, as the derivative of a Lorentzian line shape, can be described by the Bloch equation ${ }^{70}$. The peak-to-peak intensity $\left(y_{p p}^{\prime}\right)$ and line width $\left(\Delta B_{p p}\right)$ are given by ${ }^{70}$ :

$$
y_{p p}^{\prime}=\frac{y_{0}^{\prime} \cdot B_{1}}{\left(1+\gamma_{S}^{2} \cdot B_{1}^{2} \cdot T_{1 e} \cdot T_{2 e}\right)^{3 / 2}}, \quad \Delta B_{p p}=\frac{2}{\sqrt{3} \gamma_{s} \cdot T_{2 e}}\left(1+\gamma_{S}^{2} \cdot B_{1}^{2} \cdot T_{1 e} \cdot T_{2 e}\right)^{1 / 2},
$$

where $B_{1}$ is the microwave field strength $\left(B_{1}=c \sqrt{Q \cdot P_{M W}}, c^{2} \cdot Q\right.$ is the cavity conversion factor, $Q$ is the quality factor of a resonator); $y_{0}^{\prime}$ is the slope of the cure $y_{p p}^{\prime}\left(B_{1}\right)$ below saturation as deduced from the linear range of the curve; $T_{1 e}$ and $T_{2 e}$ are the electron spinlattice and spin-spin relaxation times, respectively. The correspondent analytical expression for the power dependence of the saturation factor, as reduction of the Lorentzian line intensity, is ${ }^{70}$ :

$$
s=1-\frac{1}{1+\gamma_{S}^{2} \cdot B_{1}^{2} \cdot T_{1 e} \cdot T_{2 e}} .
$$




\section{Theoretical background}

Some paramagnetic systems, for example $\mathrm{NO}^{\bullet}$ radicals, exhibit EPR spectra with several hyperfine (hf) lines due to an interaction with the coupled nuclei. Usually, it is impossible to irradiate all hyperfine lines simultaneously due to a large hf splitting. In the case of this kind of paramagnetic systems, the saturation factor $s$ in the Overhauser equation (2.9) should be replaced by the effective saturation factor $s_{\text {eff }}{ }^{73,163}$. The effective saturation factor is an arithmetic average of individual saturation factors of each EPR line.

Relaxation effects between hyperfine lines. The described model for investigation of polarization exchange between the different hyperfine EPR lines was published in [N. Enkin, G. Liu, M. d. C. Gimenez-Lopez, K. Porfyrakis, I. Tkach and M. Bennati, Phys Chem Chem Phys, 2015, 17, 11144-11149]. In the case of a nitroxide radical, three or two hyperfine lines can be observed depending on the used nitrogen isotope $\left({ }^{14} \mathrm{~N}\right.$ or $\left.{ }^{15} \mathrm{~N}\right)$. Relaxation of this system includes the electron and nitrogen nuclear spin relaxations as well as the Heisenberg spin exchange (HE) ${ }^{71,164,165}$ (Fig. 2.2). Following the initial model of Bates and Drozdoski ${ }^{71}$, the $\mathrm{HE}$ is considered as an intermolecular process, in which the individual ${ }^{14} \mathrm{~N}$ nuclear spin states as well as the total electron spin state are conserved. Within this model, the quantum states are defined as $|\mathbf{1}\rangle=|+; \mathbf{+ 1}\rangle,|\mathbf{2}\rangle=|-;+\mathbf{1}\rangle$, $|\mathbf{3}\rangle=|+; \mathbf{0}\rangle,|\mathbf{4}\rangle=|-; \mathbf{0}\rangle,|\mathbf{5}\rangle=|+;-\mathbf{1}\rangle,|\mathbf{6}\rangle=|-;-\mathbf{1}\rangle,{ }^{71,166}$ where \pm denotes the $\mathrm{m}_{\mathrm{s}}= \pm 1 / 2$ electron spin states, and 0 and \pm 1 represent the ${ }^{14} \mathrm{~N}$ nuclear states. According to this, the $\mathrm{HE}$, which is induced by a collision of two molecules with different nuclear and electron spin states, leads effectively to the following exchange of populations:

$$
\begin{aligned}
& |1\rangle+|4\rangle \longleftrightarrow \stackrel{K_{x}}{\longleftrightarrow}|2\rangle+|3\rangle \\
& |3\rangle+|6\rangle \longleftrightarrow K_{x} \longleftrightarrow|4\rangle+|5\rangle \\
& |1\rangle+|6\rangle \longleftarrow K_{x} \longrightarrow|2\rangle+|5\rangle
\end{aligned}
$$




\section{Theoretical background}

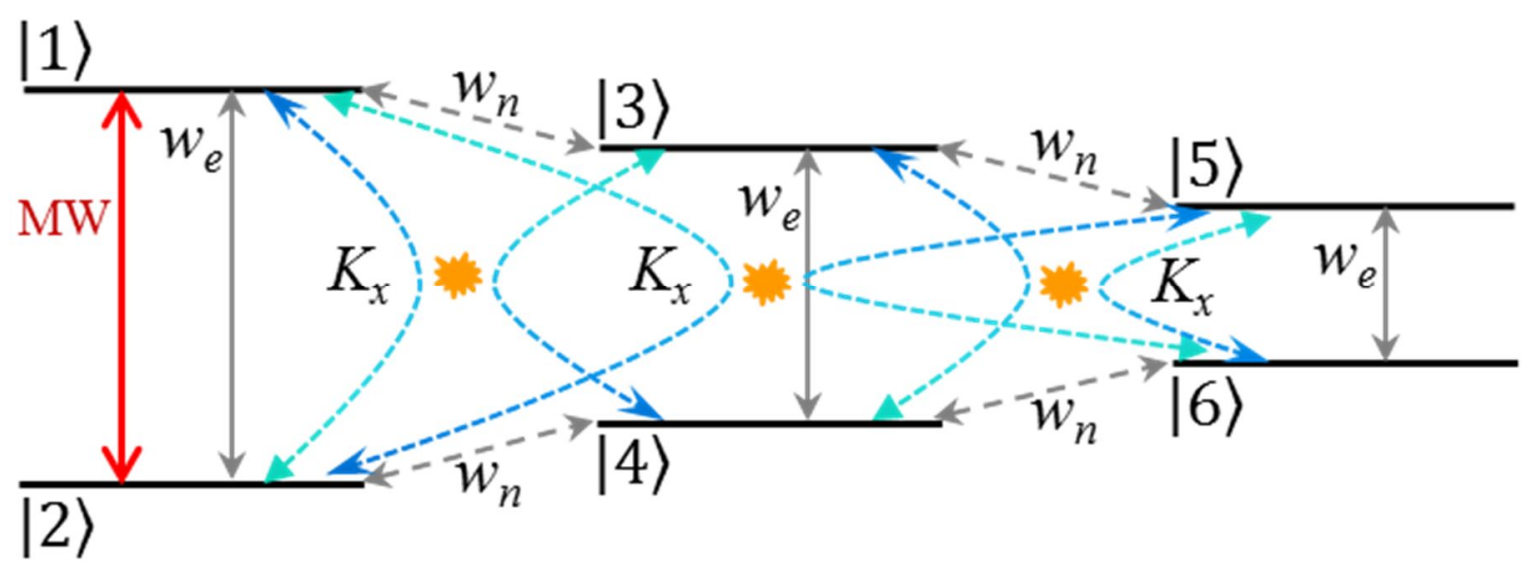

Figure 2.2: Relaxation scheme for the $S=1 / 2$ and $I=1$ coupled spin system. The red arrow indicates a continuous MW irradiation at one of the hyperfine EPR transitions. The grey solid and dashed lines indicate electron $\left(w_{e}\right)$ and ${ }^{14} \mathrm{~N}$ nuclear $\left(w_{n}\right)$ spin relaxation pathways, respectively. The blue-cyan ones (dashed) depict the HE ${ }^{163}$.

The time evolution of the six-state populations $N_{\mathrm{i}}(i=1, \ldots, 6)$ can be described by the following system of the rate equations ${ }^{163,166}$ :

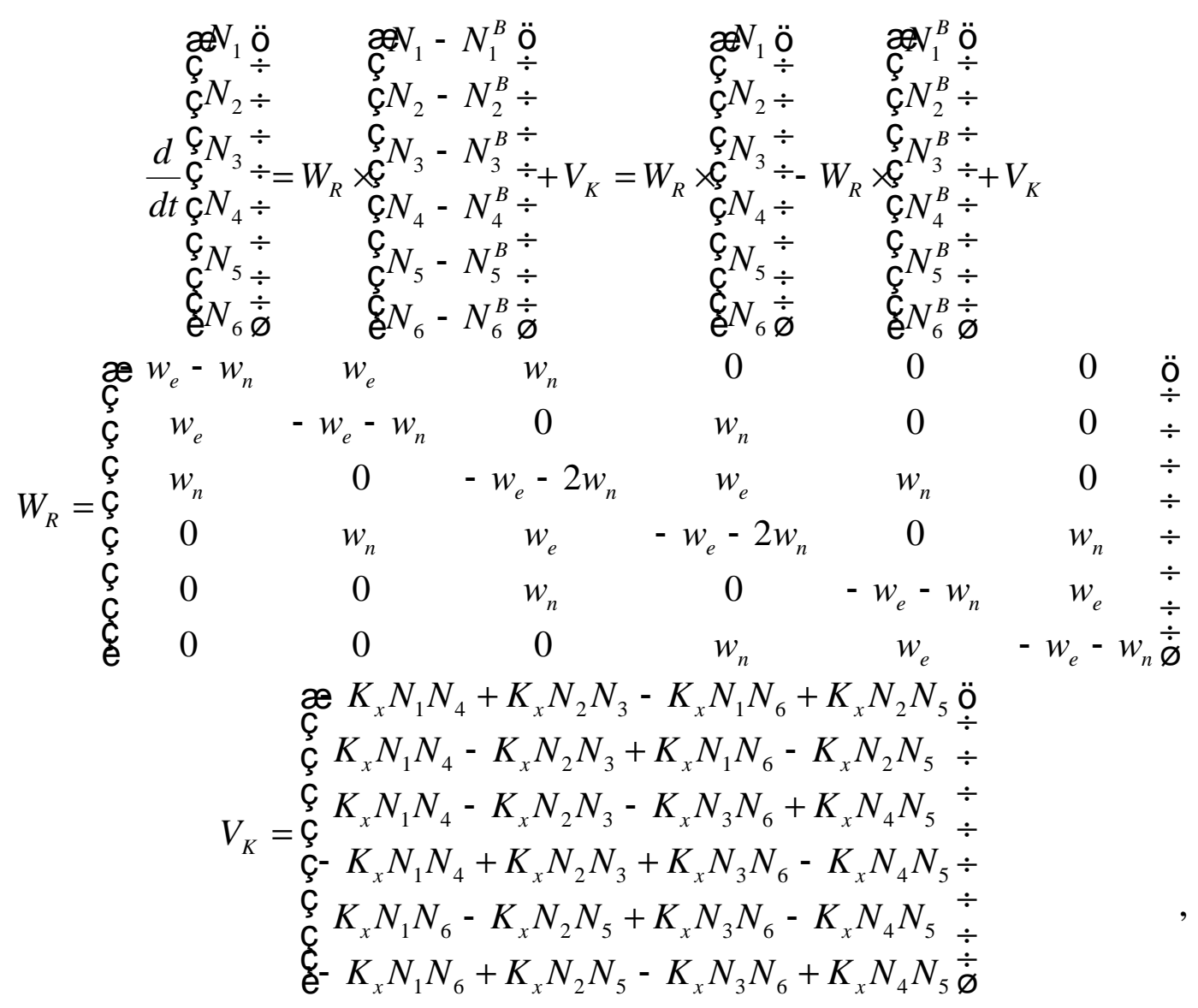




\section{Theoretical background}

where $2 w_{e}, 2 w_{n}$ are the electron and ${ }^{14} \mathrm{~N}$ nuclear relaxation rates, respectively; $K_{x}$ is the normalized Heisenberg spin exchange (HE) rate per mole, which is a second order (intermolecular) rate parameter; $W_{R}$ is the relaxation matrix and $V_{K}$ is a vector defined to account for the HE rate according to $(2.14) . N_{i}^{B}(i=1, \ldots, 6)$ correspond to the Boltzmann level populations.

The EPR signal intensity is expressed in the form of population differences:

$$
\begin{aligned}
& i_{1}=N_{2}-N_{1} \\
& i_{2}=N_{4}-N_{3}, \\
& i_{3}=N_{6}-N_{5}
\end{aligned}
$$

with the conserved total population $N=N_{1}+N_{2}+N_{3}+N_{4}+N_{5}+N_{6}=N^{B}$. Thus, with the above definition of the states, the $i_{1}$ and $i_{3}$ are the intensities of the low and high field lines, respectively. Likewise,

$$
\begin{aligned}
& i_{1}^{B}=N_{2}^{B}-N_{1}^{B} \\
& i_{2}^{B}=N_{4}^{B}-N_{3}^{B}, \\
& i_{3}^{B}=N_{6}^{B}-N_{5}^{B}
\end{aligned}
$$

where $i_{1}{ }^{B}, i_{2}{ }^{B}, i_{3}{ }^{B}$ are proportional to the EPR signals at thermal equilibrium.

To describe the time dependence of signal intensities, we perform a linear equation transformation and introduce following additional parameters: $i_{n 12}=N_{1}+N_{2}-N_{3}-N_{4}$, $i_{n 12}{ }^{B}=N_{1}{ }^{B}+N_{2}{ }^{B}-N_{3}{ }^{B}-N_{4}{ }^{B}$ (sum of NMR intensities from transitions between $m_{I}=-1$ and $m_{I}=0$ states) and $i_{n 23}=N_{3}+N_{4}-N_{5}-N_{6}, i_{n 23}{ }^{B}=N_{3}{ }^{B}+N_{4}{ }^{B}-N_{5}{ }^{B}-N_{6}{ }^{\mathrm{B}}$ (sum of NMR intensities from transitions between $m_{I}=0$ and $m_{I}=+1$ states). After the transformation, the new system of the equations reduces to: 


\section{Theoretical background}

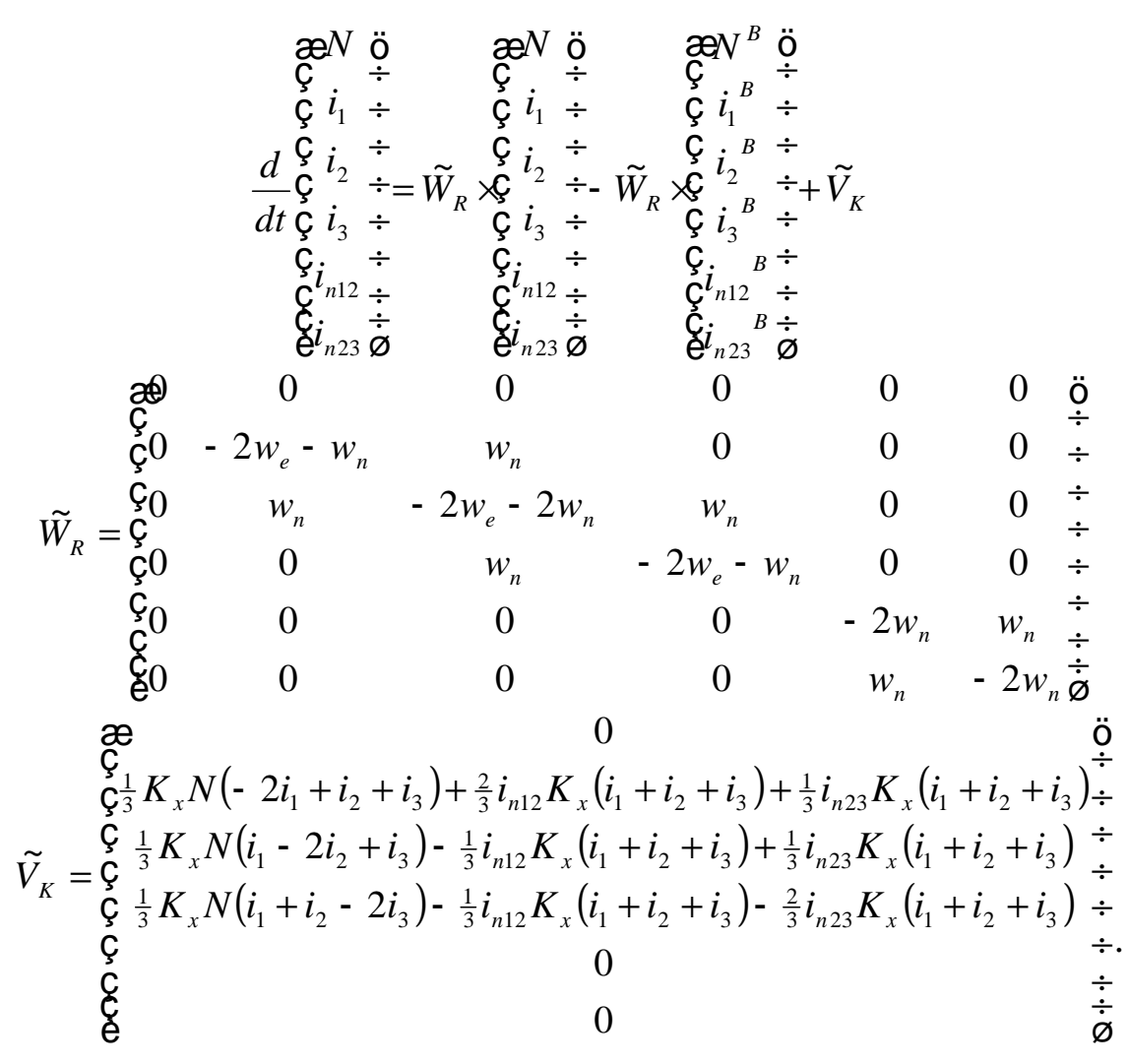

Since the ${ }^{14} \mathrm{~N}-\mathrm{NMR}$ signal terms $\left(i_{n 12}, i_{n 23}, i_{n 12}^{B}, i_{n 23}^{B}\right)$ are much smaller compared with the EPR signal $\left(i_{1}, i_{2}, i_{3}\right)$ because $\omega_{e}>\omega_{A}\left(\omega_{e} / 2 \pi \approx 9 \mathrm{GHz}\right.$ - electron Larmor frequency, $\omega_{A} / 2 \pi \approx 40 \mathrm{MHz}-$ splitting of ${ }^{14} \mathrm{~N}$ nuclear spin including the hyperfine coupling in ${ }^{14} \mathrm{NO}{ }^{\circ}$ ), all elements in $\tilde{V}_{K}$ containing the products of the NMR signal terms can be neglected. Furthermore, the total population $N$ is a constant value $\left(N^{B}=N\right)$. Thus, the system of the equations simplifies to: 


\section{Theoretical background}

$$
\begin{aligned}
& \frac{d}{d t}\left(\begin{array}{l}
i_{1} \\
i_{2} \\
i_{3}
\end{array}\right)=\tilde{W}_{R}^{*} \cdot\left(\begin{array}{l}
i_{1}-i_{1}^{B} \\
i_{2}-i_{2}^{B} \\
i_{3}-i_{3}^{B}
\end{array}\right)+\tilde{W}_{K}^{*} \cdot\left(\begin{array}{l}
i_{1} \\
i_{2} \\
i_{3}
\end{array}\right)=\left(\tilde{W}_{R}^{*}+\tilde{W}_{K}^{*}\right) \cdot\left(\begin{array}{c}
i_{1}-i_{1 B}^{*} \\
i_{2}-i_{2 B}^{*} \\
i_{3}-i_{3 B}^{*}
\end{array}\right)=W \cdot\left(\begin{array}{c}
i_{1}-i_{1 B}^{*} \\
i_{2}-i_{2 B}^{*} \\
i_{3}-i_{3 B}^{*}
\end{array}\right) \\
& \tilde{W}_{R}^{*}=\left(\begin{array}{ccc}
-2 w_{e}-w_{n} & w_{n} & 0 \\
w_{n} & -2 w_{e}-2 w_{n} & w_{n} \\
0 & w_{n} & -2 w_{e}-w_{n}
\end{array}\right) \tilde{W}_{K}^{*}=\left(\begin{array}{ccc}
-\frac{2}{3} K_{x} N & \frac{1}{3} K_{x} N & \frac{1}{3} K_{x} N \\
\frac{1}{3} K_{x} N & -\frac{2}{3} K_{x} N & \frac{1}{3} K_{x} N \\
\frac{1}{3} K_{x} N & \frac{1}{3} K_{x} N & -\frac{2}{3} K_{x} N
\end{array}\right) \\
& W=\tilde{W}_{R}^{*}+\tilde{W}_{K}^{*}=\left(\begin{array}{ccc}
-2 w_{e}-w_{n}-\frac{2}{3} K_{x} N & w_{n}+\frac{1}{3} K_{x} N & \frac{1}{3} K_{x} N \\
w_{n}+\frac{1}{3} K_{x} N & -2 w_{e}-2 w_{n}-\frac{2}{3} K_{x} N & w_{n}+\frac{1}{3} K_{x} N \\
\frac{1}{3} K_{x} N & w_{n}+\frac{1}{3} K_{x} N & -2 w_{e}-w_{n}-\frac{2}{3} K_{x} N
\end{array}\right) \\
& i_{k B}^{*}(k=1,2,3): \quad \tilde{W}_{R}^{*} \cdot\left(\begin{array}{l}
i_{1}^{B} \\
i_{2}^{B} \\
i_{3}^{B}
\end{array}\right)=W \cdot\left(\begin{array}{l}
i_{1 B}^{*} \\
i_{2 B}^{*} \\
i_{3 B}^{*}
\end{array}\right)
\end{aligned}
$$

The so-defined $i_{k B}^{*}(k=1,2,3)$ take into account also the effect of HE, which affects the Boltzmann distribution. The rate matrix $W$, which describes the time evolution of polarization recovery, can be diagonalized leading to the real eigenvalues $\left(-2 w_{e}\right.$, $\left.-2 w_{e}-3 w_{n}-K_{x} N,-2 w_{e}-w_{n}-K_{x} N\right)$ with different combinations of the relaxation rates and the eigenvectors $\left(\left(\begin{array}{l}1 \\ 1 \\ 1\end{array}\right),\left(\begin{array}{c}1 \\ -2 \\ 1\end{array}\right),\left(\begin{array}{c}1 \\ 0 \\ -1\end{array}\right)\right)$, respectively. The solution is then obtained as a sum of three exponential terms $(k=1,2,3)^{163}$ :

$$
\left(\begin{array}{l}
i_{1} \\
i_{2} \\
i_{3}
\end{array}\right)=A\left(\begin{array}{l}
1 \\
1 \\
1
\end{array}\right) e^{-2 w_{e} t}+B\left(\begin{array}{c}
1 \\
-2 \\
1
\end{array}\right) e^{-\left(2 w_{e}+3 w_{n}+K_{x} N\right) t}+C\left(\begin{array}{c}
1 \\
0 \\
-1
\end{array}\right) e^{-\left(2 w_{e}+w_{n}+K_{x} N\right) t}+\left(\begin{array}{l}
i_{1 B}^{*} \\
i_{2 B}^{*} \\
i_{3 B}^{*}
\end{array}\right)
$$

Here, the parameters $A, B, C$ are given by the initial conditions in each individual experiment. Therefore, for a good quality of the fit one can neglect the fixed amplitude ratio of the signals obtained from the eigenvectors but rather take into account their signs.

Under continuous MW irradiation in resonance with one of the EPR transitions, a steady-state of all populations with a fixed ratio of the line intensity is generated. MW affects the irradiated transition only. Analytical expressions for the saturation factors can be obtained from the kinetic equations (2.19) but reducing the number of variables. For 


\section{Theoretical background}

instance, if the first line $i_{1}$ is irradiated, the matrix row corresponding to this variable can be removed. If the microwave power is large enough, the irradiated transition reaches a complete saturation $\left(s_{k}=1\right.$, where $k$ is the number of the irradiated EPR line). The solution of the equations at this condition delivers an analytical expression for the saturation of the other two EPR transitions:

$$
\frac{d}{d t}\left(\begin{array}{l}
i_{2} \\
i_{3}
\end{array}\right)=\left(\begin{array}{l}
0 \\
0
\end{array}\right)=\left(\begin{array}{ccc}
w_{n}+\frac{1}{3} K_{x} N & -2 w_{e}-2 w_{n}-\frac{2}{3} K_{x} N & w_{n}+\frac{1}{3} K_{x} N \\
\frac{1}{3} K_{x} N & w_{n}+\frac{1}{3} K_{x} N & -2 w_{e}-w_{n}-\frac{2}{3} K_{x} N
\end{array}\right)\left(\begin{array}{l}
i_{1}^{s}-i_{1 B}^{*} \\
i_{2}^{s}-i_{2 B}^{*} \\
i_{3}^{s}-i_{3 B}^{*}
\end{array}\right),
$$

where $i_{k}^{s}(k=1,2,3)$ are the steady-state signal intensities of the EPR lines. After introducing the saturation factor $s_{k}=\frac{i_{k B}^{*}-i_{k}^{s}}{i_{k B}^{*}}\left(i_{1 B}^{*}=i_{2 B}^{*}=i_{3 B}^{*}=i_{B}^{*}\right)$ :

$$
\left(\begin{array}{c}
i_{B}^{*} \\
0 \\
0
\end{array}\right)=\left(\begin{array}{ccc}
1 & 0 & 0 \\
w_{n}+\frac{1}{3} K_{x} N & -2 w_{e}-2 w_{n}-\frac{2}{3} K_{x} N & w_{n}+\frac{1}{3} K_{x} N \\
\frac{1}{3} K_{x} N & w_{n}+\frac{1}{3} K_{x} N & -2 w_{e}-w_{n}-\frac{2}{3} K_{x} N
\end{array}\right)\left(\begin{array}{l}
s_{1} \cdot i_{B}^{*} \\
s_{2} \cdot i_{B}^{*} \\
s_{3} \cdot i_{B}^{*}
\end{array}\right)
$$

The corresponding expressions for $s_{2}$ and $s_{3}$ are:

$$
\begin{aligned}
& s_{2}=1-\frac{1}{1+\frac{1}{3} \frac{1-\left(1+\frac{3 w_{n}+K_{x} N}{2 w_{e}}\right)^{-1}}{\frac{1}{2}\left(\left(1+\frac{3 w_{n}+K_{x} N}{2 w_{e}}\right)^{-1}+\left(1+\frac{w_{n}+K_{x} N}{2 w_{e}}\right)^{-1}\right)}} \\
& s_{3}=1-\frac{1}{\frac{1}{2}\left(1+\frac{1}{3} \frac{1+\frac{1}{2}\left(1+\frac{3 w_{n}+K_{x} N}{2 w_{e}}\right)^{-1}}{\frac{1}{2}\left(1+\frac{w_{n}+K_{x} N}{2 w_{e}}\right)^{-1}}\right)}
\end{aligned}
$$

While, in the case of a complete saturation of the central line $\left(s_{2}=1\right)$ : 


$$
\begin{aligned}
& \left(\begin{array}{l}
0 \\
0
\end{array}\right)=\left(\begin{array}{ccc}
-2 w_{e}-w_{n}-\frac{2}{3} K_{x} N & w_{n}+\frac{1}{3} K_{x} N & \frac{1}{3} K_{x} N \\
\frac{1}{3} K_{x} N & w_{n}+\frac{1}{3} K_{x} N & -2 w_{e}-w_{n}-\frac{2}{3} K_{x} N
\end{array}\right)\left(\begin{array}{l}
i_{1}^{s}-i_{1 B}^{*} \\
i_{2}^{s}-i_{2 B}^{*} \\
i_{3}^{s}-i_{3 B}^{*}
\end{array}\right) \\
& \left(\begin{array}{c}
0 \\
i_{B}^{*} \\
0
\end{array}\right)=\left(\begin{array}{ccc}
-2 w_{e}-w_{n}-\frac{2}{3} K_{x} N & w_{n}+\frac{1}{3} K_{x} N & \frac{1}{3} K_{x} N \\
0 & 1 & 0 \\
\frac{1}{3} K_{x} N & w_{n}+\frac{1}{3} K_{x} N & -2 w_{e}-w_{n}-\frac{2}{3} K_{x} N
\end{array}\right)\left(\begin{array}{l}
s_{1} \cdot i_{B}^{*} \\
s_{2} \cdot i_{B}^{*} \\
s_{3} \cdot i_{B}^{*}
\end{array}\right)
\end{aligned}
$$

The expressions for $s_{1}$ and $s_{3}$ are:

$$
s_{1}=s_{3}=1-\frac{1}{1+\frac{1}{6 w_{e}}\left(3 w_{n}+K_{x} N\right)}
$$

The behaviour of the system with the $3^{\text {rd }}$ completely saturated line $\left(s_{3}=1\right)$ is described by the same equations as with those for the first case (i.e. $s_{1}=1$ ) but numbering is swapped respectively. In the case of the incomplete saturation of the irradiated EPR line, the analytical expressions described above $(2.23,2.25)$ represent the ratio of the saturation factors of the not-irradiated lines to the saturation factor of the irradiated one.

In the case of ${ }^{15} \mathrm{NO}^{\circ}$ with two hyperfine EPR transitions, analogous expressions for the time evolution of signal intensities and the saturation factor are following ${ }^{73}$ (irradiation of the $1^{\text {st }}$ transition, $\left.s_{l}=1\right)$ :

$$
\begin{aligned}
& \left(\begin{array}{l}
i_{1} \\
i_{2}
\end{array}\right)=A\left(\begin{array}{l}
1 \\
1
\end{array}\right) e^{-2 w_{e} t}+B\left(\begin{array}{c}
1 \\
-1
\end{array}\right) e^{-\left(2 w_{e}+2 w_{n}+K_{x} N\right) t}+\left(\begin{array}{l}
i_{1 B}^{*} \\
i_{2 B}^{*}
\end{array}\right) \\
& s_{2}=1-\frac{1}{1+\frac{1}{4 w_{e}}\left(2 w_{n}+K_{x} N\right)} .
\end{aligned}
$$

2.1.2. Coupling factor: relaxations in paramagnetic solution. One of the important parameters in DNP experiment is the coupling factor $\xi(2.8)$. It characterizes the nature of the interaction between polarizer (paramagnetic species) and solvent (the carrier of the nucleus which we want to polarize). The coupling factor is related to a difference between the double and the zero quantum transition rates $(2.8)^{7}$ : 


\section{Theoretical background}

$$
\xi=\frac{w_{2}-w_{0}}{2 w_{I}+w_{0}+w_{2}}=\frac{\sigma}{\rho}=\frac{\sigma_{d i p}+\sigma_{\text {scal }}}{\rho_{\text {dip }}+\rho_{\text {scal }}}, \quad \rho_{\text {scal }}=-\sigma_{\text {scal }} .
$$

According to the spectral density theory of a molecular motion, the rates of transitions between quantum states depends on the spectral density function $J(\omega, \tau)$ for the observed motion ${ }^{69,81,167}$. In the simplest case (inner-sphere model ${ }^{69,168}$ ), the Lorentzian spectral density function is:

$$
J(\omega, \tau)=\frac{\tau}{1+\omega^{2} \tau^{2}}
$$

Thus, the transition probabilities in the DNP system can be written as 7,69

$$
\begin{aligned}
& 2 w_{I}=3 k \cdot J\left(\omega_{I}, \tau_{d i p}\right) \\
& w_{0}=k \cdot J\left(\omega_{I}+\omega_{S}, \tau_{d i p}\right)+k_{\text {scal }} \cdot J\left(\omega_{I}+\omega_{S}, \tau_{s c a l}\right), \\
& w_{2}=6 k \cdot J\left(\omega_{I}-\omega_{S}, \tau_{d i p}\right) \\
\rho_{\text {scal }}= & -\sigma_{\text {scal }}=k_{\text {scal }} \cdot J\left(\omega_{I}+\omega_{S}, \tau_{\text {scal }}\right) \\
\sigma_{\text {dip }}= & k \cdot\left(6 \cdot J\left(\omega_{I}-\omega_{S}, \tau_{d i p}\right)-J\left(\omega_{I}+\omega_{S}, \tau_{d i p}\right)\right) \\
\rho_{\text {dip }}= & k \cdot\left(3 \cdot J\left(\omega_{I}, \tau_{d i p}\right)+6 \cdot J\left(\omega_{I}-\omega_{S}, \tau_{d i p}\right)+J\left(\omega_{I}+\omega_{S}, \tau_{d i p}\right)\right)
\end{aligned}
$$

where $\omega_{S}$ and $\omega_{I}$ are the electron and nuclear Larmor frequency; $\tau_{d i p}$ and $\tau_{\text {scal }}$ are the correlation times for dipolar and scalar (Fermi contact) interactions between electron and nuclear spins; $k$ and $k_{\text {scal }}$ are constant factors for the corresponding interactions. The dipolar interaction is modulated by molecular rotational motion, the electron spin exchange (electron relaxation) and molecular exchange (as result of any interaction between nucleus-bearing and paramagnetic molecules). So that the correlation time of the dipolar interaction is determined by the characteristic times of those interactions $\left(\tau_{r}, \tau_{e}\right.$, $\tau_{m}$, respectively) ${ }^{69,167}$. The scalar interaction is modulated by the same phenomena except for the molecular reorientation. Thereby the correlation times of the interactions are:

$$
\tau_{d}^{-1}=\tau_{r}^{-1}+\tau_{e}^{-1}+\tau_{m}^{-1}, \quad \tau_{s}^{-1}=\tau_{e}^{-1}+\tau_{m}^{-1}
$$




\section{Theoretical background}

Since the nuclear Larmor frequency is much lower than the electron Larmor frequency, the expression for the paramagnetic relaxation $R_{I \text { para }}$ is:

$$
\begin{aligned}
& R_{I \text { para }}=2 w_{I}+w_{0}+w_{2}=R_{I \text { dip }}+R_{\text {I scal }} \\
& R_{I \text { dip }}=k \cdot\left(3 \cdot J\left(\omega_{I}, \tau_{\text {dip }}\right)+7 \cdot J\left(\omega_{S}, \tau_{\text {scal }}\right)\right) . \\
& R_{I \text { scal }}=k_{\text {scal }} \cdot J\left(\omega_{S}, \tau_{\text {scal }}\right)
\end{aligned}
$$

It was suggested by Stehlik ${ }^{7}$ that scalar coupling leads to a hyperfine perturbation of Zeeman states of the electron-nucleus system and, consequently, the electron relaxation transitions induce nuclear relaxation transitions. Thus, the scalar part of the relaxation $\rho_{\text {scal }}(2.29)$ includes $J\left(\omega_{I}, \tau_{\text {scal }}\right)$ with a weighting factor $\beta$ (which can have any values between 0 and 1) ${ }^{7,69}$ :

$$
\rho_{\text {scal }}=k_{\text {scal }} \cdot\left(J\left(\omega_{S}, \tau_{\text {scal }}\right)+\beta \cdot J\left(\omega_{I}, \tau_{\text {scal }}\right)\right), \quad \sigma_{\text {scal }}=-k_{\text {scal }} \cdot J\left(\omega_{S}, \tau_{\text {scal }}\right) .
$$

In the extreme case, when the electron spin relaxation time is much larger than the correlation time of the molecular exchange, this factor $\beta$ can be negligible. For example, this condition is true for systems with free radicals as polarizers ${ }^{81}$.

Finally, the coupling factor can be expressed as:

$$
\xi=\frac{5 k \cdot J\left(\omega_{S}, \tau_{\text {dip }}\right)-k_{\text {scal }} \cdot J\left(\omega_{S}, \tau_{\text {scal }}\right)}{R_{\text {I para }}}
$$

In the case of a purely dipolar interaction $\left(k_{\text {scal }}=0\right)$, the coupling factor

$$
\xi=\frac{5 \cdot J\left(\omega_{S}, \tau_{d i p}\right)}{7 \cdot J\left(\omega_{S}, \tau_{d i p}\right)+3 \cdot J\left(\omega_{I}, \tau_{d i p}\right)}=\frac{5}{7} \cdot\left(1-\frac{2 w_{I}}{T_{1 I}^{-1}-R_{I 0}}\right)
$$

can have any value between $0\left(\omega \cdot \tau_{\text {dip }} A E \infty, \omega_{S}>\omega_{I}\right)$ and $0.5\left(\omega \cdot \tau_{\text {dip }} A E 0\right)$. In the case of a purely scalar interaction $(k=0)$, the coupling factor

$$
\xi=\frac{-J\left(\omega_{S}, \tau_{\text {scal }}\right)}{J\left(\omega_{S}, \tau_{s c a l}\right)+\beta \cdot J\left(\omega_{I}, \tau_{s c a l}\right)}=-\frac{w_{0}}{T_{1 I}^{-1}-R_{I 0}}=-1+\frac{\beta}{3} \cdot \frac{2 w_{I}}{T_{1 I}^{-1}-R_{I 0}}
$$




\section{Theoretical background}

can have any values between $-1\left(\omega \cdot \tau_{\text {scal }} \mathcal{A E} 0\right.$; if $\beta=0 \xi$ is always -1$)$ and $0\left(\omega \cdot \tau_{\text {scal }} \mathcal{A E} \infty\right.$, $\left.\beta \neq 0, \omega_{S}>\omega_{I}\right)$. The dipolar and scalar mechanisms of the interaction between the electron and the nuclear spins lead to different signs of a DNP enhancement comparing to a thermal equilibrium, i.e. minus and plus, respectively. Thus, the dipolar and scalar mechanisms counteract each other.

The model described above for the coupling factor $\xi$ corresponds to a system with a fixed distance between the nucleus and the paramagnetic molecule. The models including the translational diffusion of the molecules (outer-sphere models) have been developed and analysed in works of Abragam and Freed ${ }^{76}$. The diffusion correlation time depends on the distance of the closest approach between nucleus and electron $d$ and the diffusion coefficients of both the nucleus bearing molecule $D_{L}$ and the paramagnetic molecule $D_{P}$ (which are in turn depend on molecular sizes)

$$
\tau_{D}=\frac{d^{2}}{D_{L}+D_{R}}
$$

The relaxations of the quantum states for the diffusional model ${ }^{76,81}$ can be obtained by the same expressions which are described above:

$$
\begin{aligned}
& 2 w_{I}=3 k^{\prime} \cdot \tilde{J}\left(\omega_{I}, \tau_{D}\right) \\
& w_{0}=k^{\prime} \cdot \tilde{J}\left(\omega_{I}+\omega_{S}, \tau_{D}\right), \\
& w_{2}=6 k^{\prime} \cdot \tilde{J}\left(\omega_{I}-\omega_{S}, \tau_{D}\right)
\end{aligned}
$$

but with a different spectral density function

$$
9\left(\omega, \tau_{D}\right)=\frac{1+5 z / 8+z^{2} / 8}{1+z+z^{3} / 6+4 z^{4} / 81+z^{5} / 81+z^{6} / 648}, \quad z=\sqrt{2 \omega \tau_{D}}
$$

When the correlation time of the dipolar interaction $\tau_{\text {dip }}(2.30)$ is the same or smaller than the diffusional correlation time $\tau_{D}$ (2.36) and the distance of the closest approach (2.36) is similar to the distance of the bound nuclei, the contribution of the diffusion cannot be neglected and should be added to the model with the fixed distance between 


\section{Theoretical background}

nucleus-bearing and paramagnetic molecules ${ }^{81}$. In this more general case, the paramagnetic relaxation (Fig. 2.3) is:

$$
\begin{aligned}
& R_{I \text { para }}=R_{I \text { dip }}+R_{I \text { scal }}+R_{\text {Idiff }} \\
& R_{I \text { diff }}=k^{\prime} \cdot\left(3 \cdot \tilde{J}\left(\omega_{I}, \tau_{D}\right)+7 \cdot \tilde{J}\left(\omega_{S}, \tau_{D}\right)\right)
\end{aligned}
$$

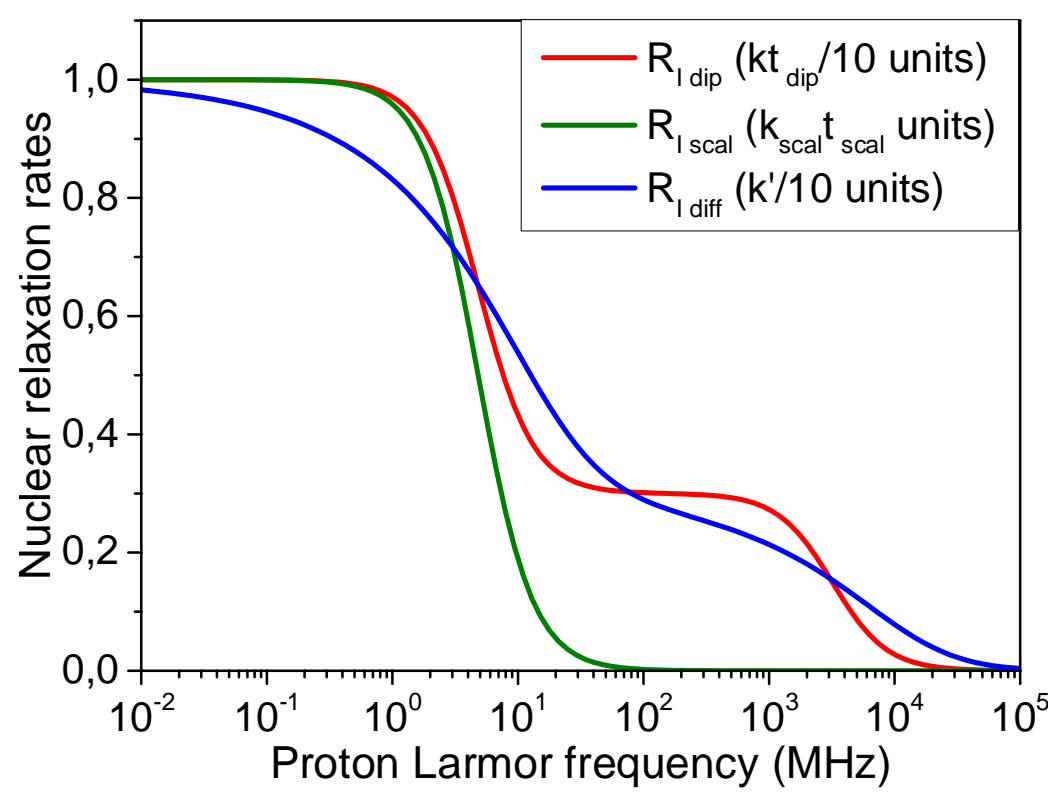

Figure 2.3: The nuclear relaxation rates as a function of the applied magnetic field (calculated for a correlation/diffusional time of $50 \mathrm{ps})^{81}$.

The coupling factor in the case including the translational diffusion (and $\beta=0$ ) is:

$$
\xi=\frac{5}{7} \cdot\left(1-\frac{3 k \cdot J\left(\omega_{I}, \tau_{c}\right)+3 k^{\prime} \cdot \tilde{J}\left(\omega_{I}, \tau_{D}\right)}{R_{\text {I para }}}\right)-\frac{12}{7} \cdot \frac{R_{I \text { scal }}}{R_{\text {I para }}}
$$

Fig. 2.4 shows the magnetic field dependence of the coupling factor for different relaxation mechanisms. 


\section{Theoretical background}

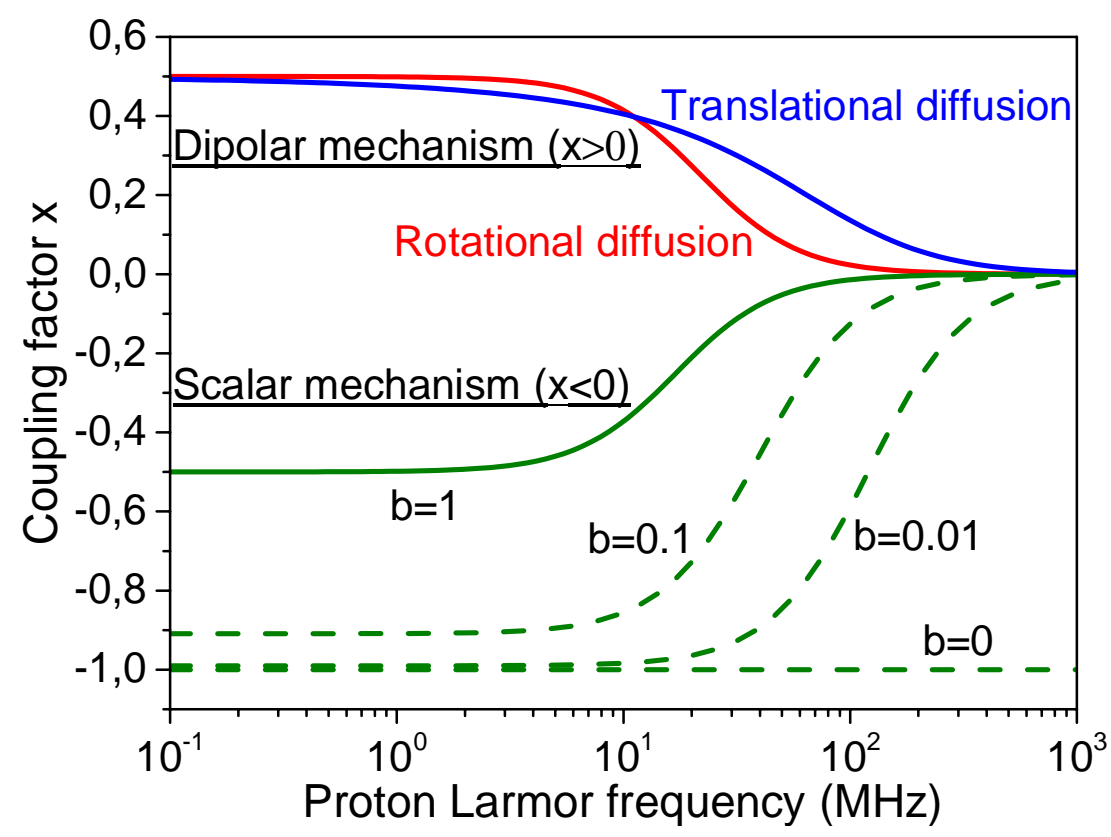

Figure 2.4: The field dependence of coupling factor for different relaxation mechanisms (calculated for a correlation/diffusional time of $20 \mathrm{ps})^{69,76}$.

Since the coupling factor is determined by the relaxation behaviour which depends on the external magnetic field, it can be measured with nuclear magnetic resonance dispersion (NMRD) experiment (Fig. 2.5) ${ }^{81,169,170}$. In the case of the negligible scalar contribution to the relaxation rate (for example, nitroxide radical) $(2.35,2.40)$ the high field relaxation limit $2 w_{I}$ can be estimated as $3 / 10$ of the low field $R_{I \text { para }}$. At $15 \mathrm{MHz}$ and $400 \mathrm{MHz}$, the values of the coupling factor between TEMPO radical $(1 \mathrm{mM})$ and toluene protons are around 0.303 and 0.123 , respectively (Fig. 2.5). 


\section{Theoretical background}

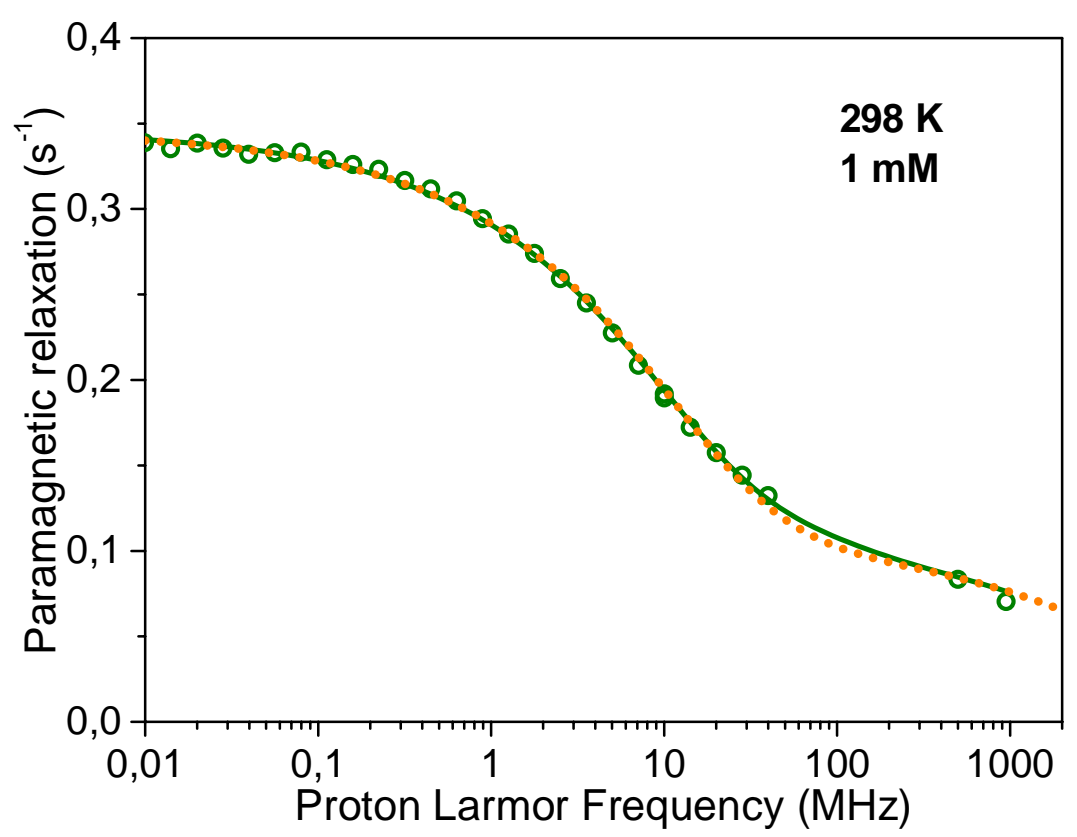

Figure 2.5: The NMRD profile of the paramagnetic relaxation $\left(R_{I \text { para }}\right)$ for toluene protons with $1 \mathrm{mM}$ of TEMPONE at $298 \mathrm{~K}$. Orange dotted line: outer-sphere model only fitting $(d=3.25 \AA$, $D=2.46 \times 10^{-9} \mathrm{~m}^{2} / \mathrm{s}, \tau_{D}=43 \mathrm{ps}$ ); Green solid line: inner-sphere and outer-sphere models together $\left(d=3.45 \AA, D=2.42 \times 10^{-9} \mathrm{~m}^{2} / \mathrm{s}, \tau_{\text {dip }}=43 \mathrm{ps}\right)$.

The validity of the pure dipolar interaction can be proved with the ratio of the high field relaxation to the law filed one which should be $3 / 10^{81}$.

2.1.3. Leakage factor. The leakage factor $f(2.8)$ is related to the ratio of spin-lattice relaxations of nucleus in the presence $\left(T_{1 I}^{-1}\right)$ and the absence $\left(T_{1 I 0}^{-1}\right)$ of the paramagnetic compound:

$$
f=\frac{2 w_{I}+w_{0}+w_{2}}{2 w_{I}+w_{0}+w_{2}+R_{I 0}}=\frac{R_{I}-R_{I 0}}{R_{I}}=\frac{R_{I \text { para }}}{R_{I}}=\frac{R \cdot n}{R_{I}}=1-\frac{T_{1 I}}{T_{1 I 0}}
$$

with $R_{I \text { para }}=R \cdot n$, where $R$ is the relaxivity, $n$ is concentration of the paramagnetic compound. The leakage factor can be obtained from the inversion recovery experiment by measuring nuclear relaxations of the investigated system with and without paramagnetic compound. In the case of the absence of a pure solution with the nucleusbearing molecule, the leakage factor can be obtained from the above described experiment for the observed solution with different concentrations. The optimal fitting of 


\section{Theoretical background}

the linear function $R_{I}=R_{I O}+R \cdot n$ gives $R_{I O}$ and $R$ values. The maximum asymptotic value of the leakage factor is 1 , which usually can be reached at high radical concentrations.

\subsection{Radical-dye system}

The interaction of a free radical with a photoexcited triplet state leads to hyperpolarization of the radical. The effect is based on the transfer of a non-equilibrium spin polarization from the triplet state, which is formed by intersystem crossing (ISC), to the ground state of the radical ${ }^{147,158}$. This so-called dynamic electron polarization (DEP) (Fig. 2.6), occurs in both cases when the dye, as a carrier of the triplet, is covalently linked ${ }^{171,172}$ to the radical and when it is not bound but just colliding with the radical in a liquid solution ${ }^{173-175}$.

\section{Triplet state}

\section{Electron polarization}

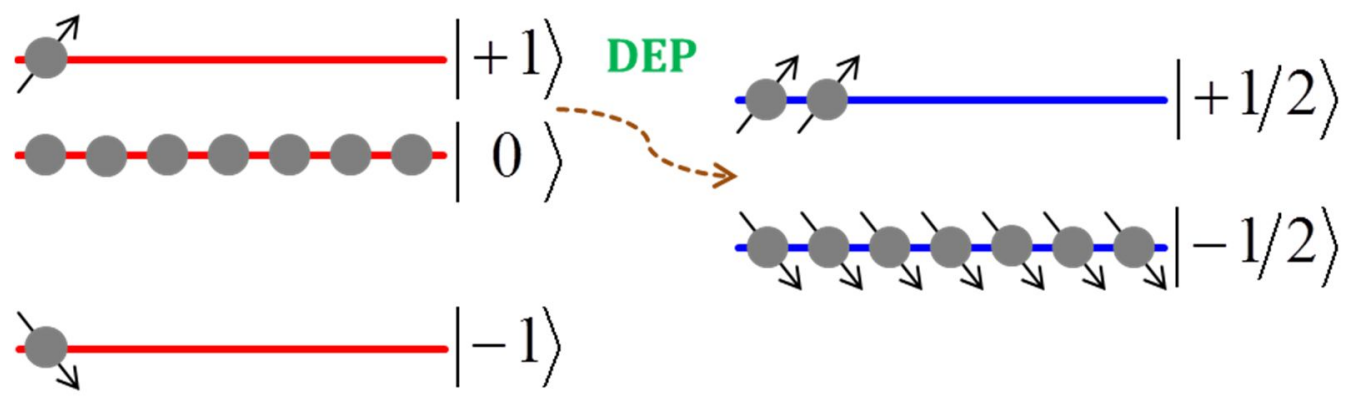

Figure 2.6: Scheme of dynamic electron polarization (DEP) concept.

2.2.1. Photoexcited triplet state. A system with two electrons $(S=1 / 2)$ has four possible combinations of the spin states

$$
|-1 / 2\rangle|-1 / 2\rangle \quad|-1 / 2\rangle|+1 / 2\rangle \quad|+1 / 2\rangle|-1 / 2\rangle \quad|+1 / 2\rangle|+1 / 2\rangle .
$$

These combinations give three basis states $\left|\mathrm{S}, \mathrm{S}_{\mathrm{z}}\right\rangle$ with the total spin angular momentum $\boldsymbol{I}$ (triplet) and one with the total spin angular momentum $\boldsymbol{O}$ (singlet):

$$
\begin{aligned}
& |1,-1\rangle=|-1 / 2\rangle|-1 / 2\rangle \\
& |1,0\rangle=(|-1 / 2\rangle|+1 / 2\rangle+|+1 / 2\rangle|-1 / 2\rangle) / \sqrt{2} \quad|0,0\rangle=(|-1 / 2\rangle|+1 / 2\rangle-|+1 / 2\rangle|-1 / 2\rangle) / \sqrt{2} . \\
& |1,+1\rangle=|+1 / 2\rangle|+1 / 2\rangle
\end{aligned}
$$




\section{Theoretical background}

Light with the photon energy matching the optical transition excites the molecule from the ground state $\mathbf{S}_{\mathbf{0}}$ to the excited singlet state $\mathbf{S}_{\mathbf{1}}$ (Fig. 2.7). After that, the electronic state of the excited molecule either relax back to the ground state $\mathbf{S}_{\mathbf{0}}$ emitting photons or relax to the lowest triplet state $\mathbf{T}_{\mathbf{1}}$ due to spin-orbital coupling ${ }^{176}$. The first process is called a fluorescence and the second one is the intersystem crossing (ISC) ${ }^{176}$. Photons can be also emitted on the transition from the excited triplet state $\mathbf{T}_{\mathbf{1}}$ to the ground state $\mathbf{S}_{\mathbf{0}}$, however with a much slower rate in contrast to fluorescence since these transitions are forbidden. This kind of light emission is called phosphorescence ${ }^{176}$. Since the transition probabilities from the excited singlet $\mathbf{S}_{\mathbf{1}}$ to three sublevels of the triplet $\mathbf{T}_{\mathbf{1}}$ are different the ISC generates the population of $\mathbf{T}_{\mathbf{1}}$ state which differs from thermal equilibrium ${ }^{147}$, ${ }^{176}$. Thus, the excited triplet usually exhibits higher electron spin polarization than that of Boltzmann equilibrium independently on the temperature and the external magnetic field. This polarization of the photoexcited triplet state is affected by the structure of a given molecule.

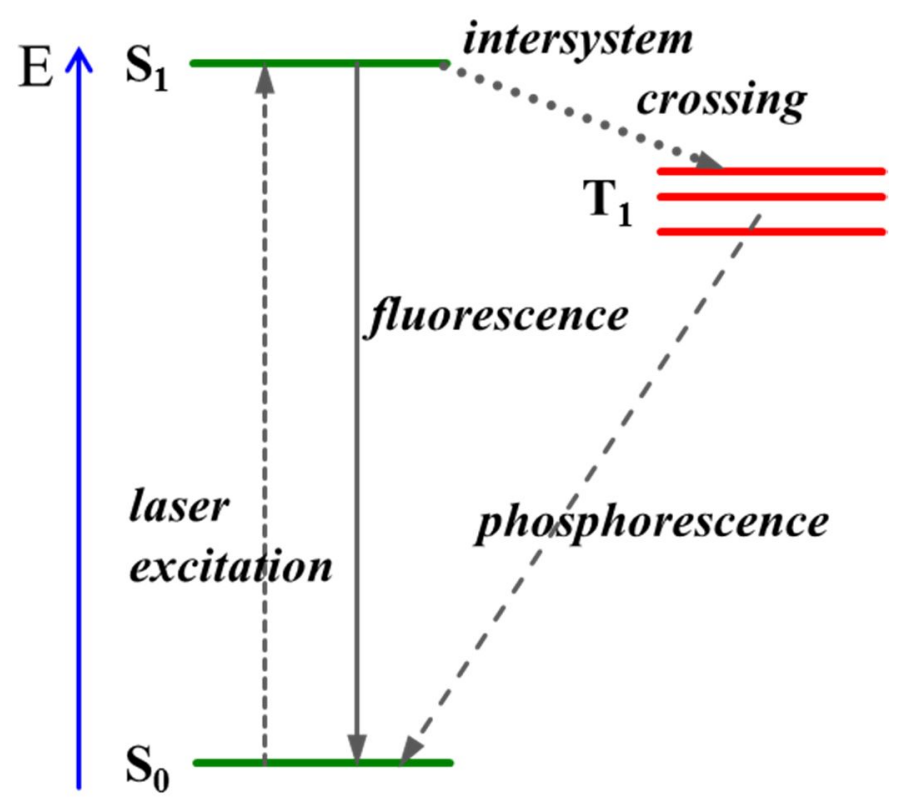

Figure 2.7: Jablonski diagram of a molecule which can be excited to the triplet state.

The quantum yield and the lifetime of an excited triplet are important characteristics of a certain dye. The quantum yield depends on the transition rates involved. The populations of the states $\left(S_{0}, S_{1}, T_{1}\right)$ in the excited molecule can be described by the following kinetic equation ${ }^{176}$ : 


\section{Theoretical background}

$$
\frac{d}{d t}\left(\begin{array}{l}
S_{0} \\
S_{1} \\
T_{1}
\end{array}\right)=\left(\begin{array}{ccc}
-k_{\text {exc }} & k_{f} & k_{p} \\
k_{\text {exc }} & -k_{f}-k_{i s c} & 0 \\
0 & k_{i s c} & -k_{p}
\end{array}\right)\left(\begin{array}{l}
S_{0} \\
S_{1} \\
T_{1}
\end{array}\right)\left(\begin{array}{l}
S_{0} \\
S_{1} \\
T_{1}
\end{array}\right)_{t=0}=\left(\begin{array}{l}
1 \\
0 \\
0
\end{array}\right) \quad S_{0}+S_{1}+T_{1}=1,(2.4
$$

where $k_{f}, k_{i s c}, k_{p}$ are the rates of fluorescence, intersystem crossing and phosphorescence, respectively. The excitation rate $k_{e x c}$ is given by $k_{e x c}=I_{0} \cdot \sigma_{g} / h \omega$, where $\sigma_{g}$ is the excitation cross section, $I_{0}=P_{L} / \sigma$ is density of the excitation (the ratio of the excitation power $P_{L}$ and area of a cross section of the excitation beam $\sigma$ ). The steady state $\left(T_{1}^{s}\right)$ and the quantum yield $\left(Y_{T 1}\right)$ of the triplet can be expressed from the equation (2.44):

$$
T_{1}^{s}=\frac{1}{1+k_{p} \cdot\left(\frac{1}{k_{e x c}}+\frac{1}{k_{i s c}}+\frac{k_{f}}{k_{e x c} \cdot k_{i s c}}\right)}, \quad Y_{T 1}=\frac{1}{1+k_{p} / k_{i s c}} \text {. }
$$

The steady state $T_{1}^{s}$ increases with the lifetime of the state $\left(\tau_{p}=1 / k_{p}\right)$, the ISC rate and the power of light excitation (Fig. 2.8). The quantum yield does not depend on the power.

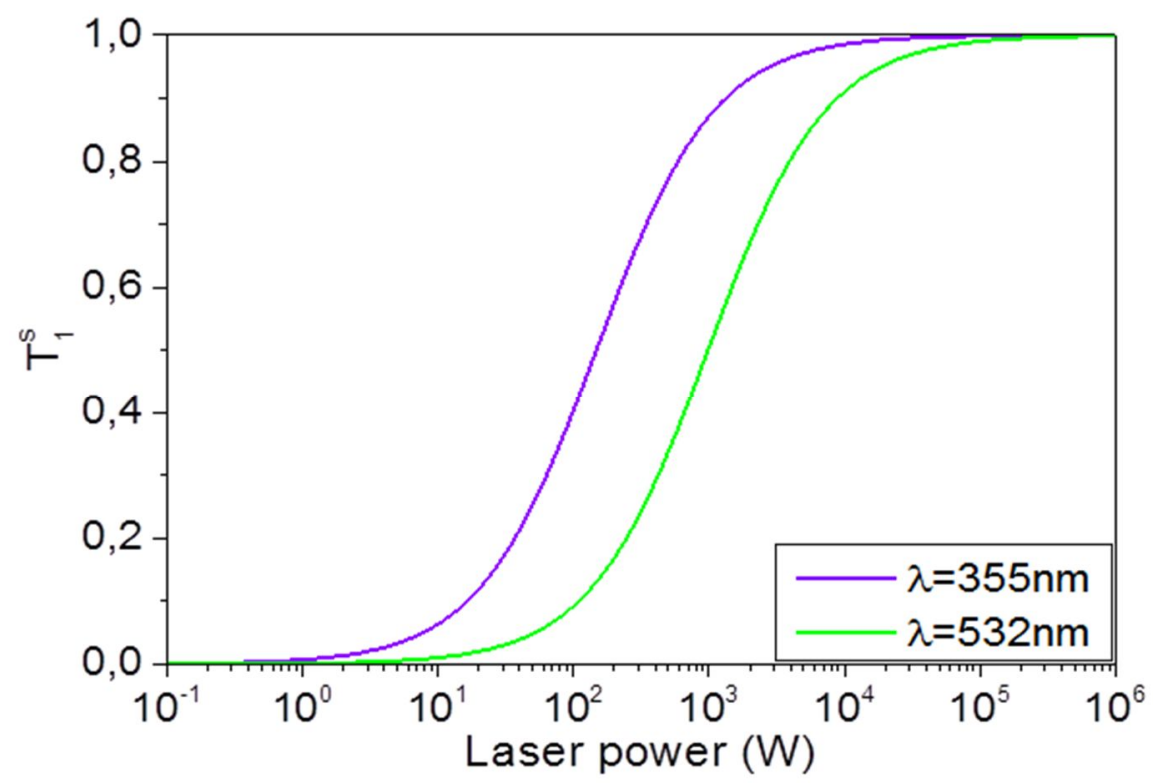

Figure 2.8: The laser power dependence of the photoexcited triplet state for fullerene $\mathrm{C}_{60}$ (laser beam section $\sigma=5 \mathrm{~mm}^{2}, k_{f} \sim 1 \mathrm{~ns}^{-1}, k_{i s c}=0.143 \mathrm{ps}^{-1}, \tau_{p}=5 \mu \mathrm{s}$ ). The quantum yield is around unity $\left(Y_{T l}=0.993\right)$. 


\section{Theoretical background}

2.2.2. States of radical-dye system. A radical-dye system with a fixed distance (for example, covalently linked $\mathrm{C}_{60}$-TEMPO ${ }^{161,162,171}$ ) can be described by the interaction between a doublet $(S=1 / 2)$ of a radical and singlets $(S=0)$ and a triplet $(S=1)$ of a dye. The combination of the radical doublet with the dye singlets $\left(\mathbf{S}_{\mathbf{0}}\right.$ and $\mathbf{S}_{\mathbf{1}}$ ) produces two new doublets $\left|\mathrm{S}, \mathrm{S}_{\mathrm{Z}}\right\rangle\left(\mathbf{D}_{\mathrm{S} 0}\right.$ and $\left.\mathbf{D}_{\mathrm{S} 1}\right)$

$$
\left.\begin{array}{l}
|1 / 2,-1 / 2\rangle|0,0\rangle \\
|1 / 2,+1 / 2\rangle|0,0\rangle
\end{array}\right\} \Rightarrow \begin{aligned}
& |1 / 2,-1 / 2\rangle \\
& |1 / 2,+1 / 2\rangle
\end{aligned}
$$

The radical doublet with the dye triplet produces six combinations ${ }^{177}$

$$
\begin{array}{lll}
|1 / 2,-1 / 2\rangle|1,-1\rangle & |1 / 2,-1 / 2\rangle|1,0\rangle & |1 / 2,-1 / 2\rangle|1,+1\rangle \\
|1 / 2,+1 / 2\rangle|1,-1\rangle & |1 / 2,+1 / 2\rangle|1,0\rangle & |1 / 2,+1 / 2\rangle|1,+1\rangle
\end{array},
$$

which gives four basis states with the total spin angular momentum 3/2 (quartet $\mathbf{Q}_{\mathbf{T} 1}$ ) and two basis states with total spin angular momentum $1 / 2$ (doublet $\left.\mathbf{D}_{\mathrm{T} 1}\right)\left(\mathbf{F i g}\right.$. 2.9) ${ }^{177}$

$$
\begin{aligned}
& |3 / 2,-3 / 2\rangle=|1 / 2,-1 / 2\rangle|1,-1\rangle \\
& |3 / 2,-1 / 2\rangle=(|1 / 2,+1 / 2\rangle|1,-1\rangle+\sqrt{2}|1 / 2,-1 / 2\rangle|1,0\rangle) / \sqrt{3} \\
& |3 / 2,+1 / 2\rangle=(\sqrt{2}|1 / 2,+1 / 2\rangle|1,0\rangle+|1 / 2,-1 / 2\rangle|1,+1\rangle) / \sqrt{3} \\
& |3 / 2,+3 / 2\rangle=|1 / 2,+1 / 2\rangle|1,+1\rangle \\
& |1 / 2,-1 / 2\rangle=(\sqrt{2}|1 / 2,+1 / 2\rangle|1,-1\rangle-|1 / 2,-1 / 2\rangle|1,0\rangle) / \sqrt{3} \\
& |1 / 2,+1 / 2\rangle=(\sqrt{2}|1 / 2,-1 / 2\rangle|1,+1\rangle-|1 / 2,+1 / 2\rangle|1,0\rangle) / \sqrt{3}
\end{aligned}
$$

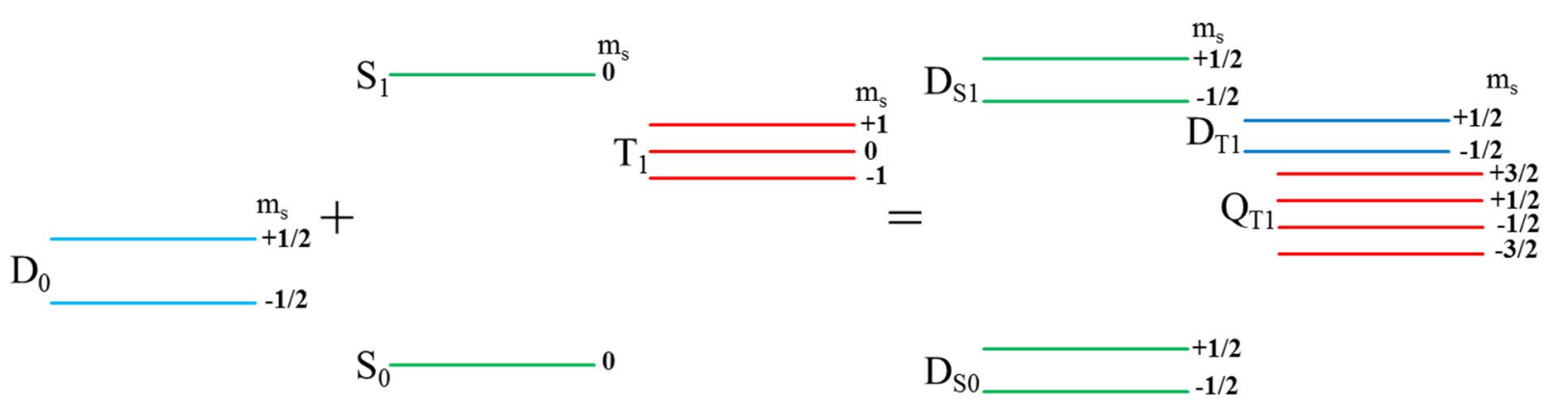

Figure 2.9: Scheme of the superposition of a radical doublet $\left(\mathbf{D}_{\mathbf{0}}\right)$ with singlets and triplet states of a dye ( $\mathbf{S}_{\mathbf{0}}, \mathbf{S}_{\mathbf{1}}$ and $\mathbf{T}_{\mathbf{1}}$, respectively). 


\section{Theoretical background}

The Hamiltonian of the system can be represented as a sum of two spin exchange interactions. The first one is the interaction between two electrons of the dye $\left(S_{a}\right.$ and $\left.S_{b}\right)$ and the second one is between the radical electron $\left(S_{R}\right)$ and the triplet of the dye $\left(S_{T}=S_{a}+S_{b}\right)$ :

$$
H_{e x}^{D D}=-2 J_{D D} \cdot S_{a} \cdot S_{b} \quad H_{e x}^{R T}=-2 J_{R T} \cdot S_{R} \cdot S_{T},
$$

where $J_{D D}$ and $J_{R T}$ are the exchange integrals (Fig. 2.10), $S_{a}, S_{b}$ and $S_{R}$ are spin operators of the corresponding electrons.

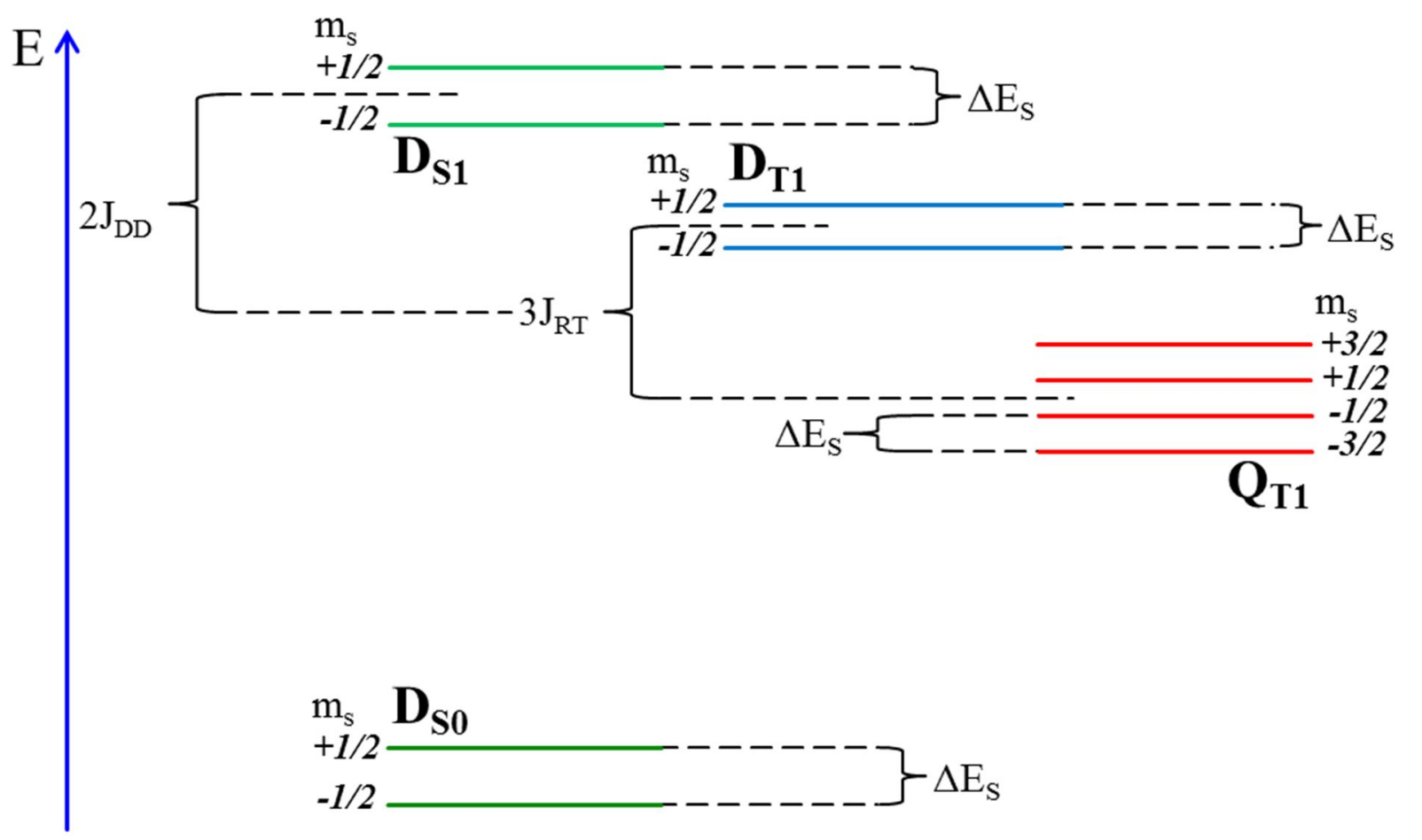

Figure 2.10: Jablonski diagram of the radical-dye system ${ }^{154,177}$.

The light excitation transfers the electrons of the radical-dye molecule from the ground doublet state $\mathbf{D}_{\mathbf{S} 0}$ to the excited doublet $\mathbf{D}_{\mathbf{S} \mathbf{1}}(\mathbf{F i g}$. 2.10). After the excitation, the electrons either return back to the ground state $\mathbf{D}_{\mathbf{S} 0}$ or are transferred to the doublet $\mathbf{D}_{\mathbf{T} 1}$ and the quartet $\mathbf{Q}_{\mathbf{T} 1}$ due to ISC, which occurs within sublevels of the radical-dye coupled system (Fig. 2.10). 


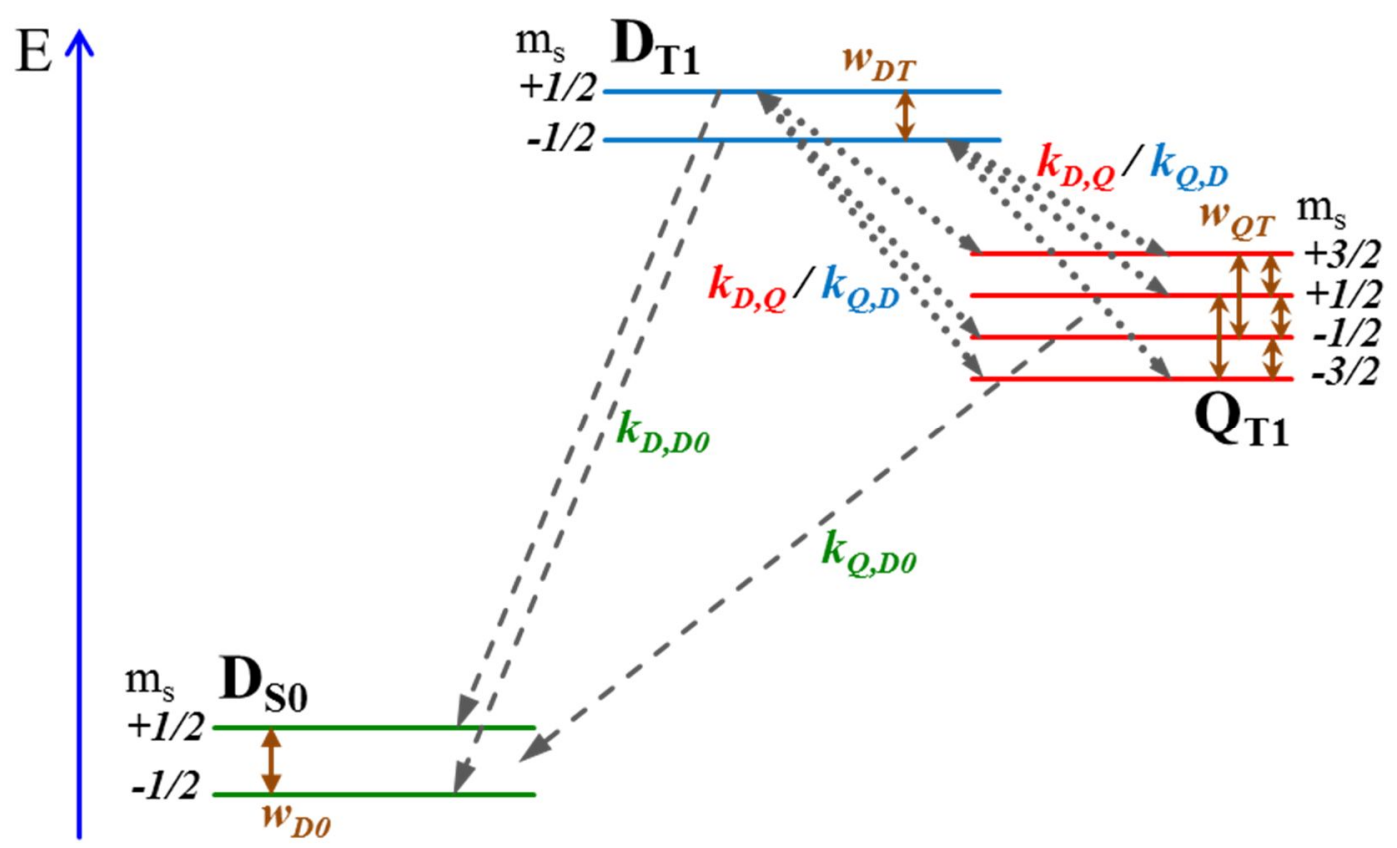

Figure 2.11: Kinetic scheme of processes in the radical-dye system according to the model of the reversed quartet mechanism ${ }^{154} . k_{D, Q}$ and $k_{Q, D}$ are rates of transitions from $\mathbf{D}_{\mathbf{T} 1}$ to $\mathbf{Q}_{\mathbf{T} 1}$ and from $\mathbf{Q}_{\mathbf{T} 1}$ to $\mathbf{D}_{\mathbf{T} 1}$, respectively. $k_{D, D 0}$ is the effective rate of $\mathbf{D}_{\mathbf{T} 1} \rightarrow \mathbf{D}_{\mathbf{S} 0}$ which includes several processes ${ }^{154} \cdot w_{i}(i=D O, D T, Q T)$ is transition probability between Zeeman sublevels of the corresponding states. $k_{Q, D O}$ is an ISC rate of $\mathbf{Q}_{\mathbf{T} 1} \rightarrow \mathbf{D}_{\mathbf{S 0} \text {. }}$

2.2.3. Reversed quartet mechanism. To explain the transient EPR (TrEPR) patterns in fullerene-nitroxide derivatives Corvaja et al. proporsed the reversed quartet mechanism $(\mathrm{RQM}){ }^{154}$. Reversed quartet mechanism is based on a selective depletion of quartet $\left(\mathbf{Q}_{\mathrm{T1}}\right)$ sublevels to the doublet ground state $\left(\mathbf{D}_{\mathbf{S 0}}\right)$ through reversible ISC between the doublet $\mathbf{D}_{\mathrm{T} 1}$ and the quartet $\mathbf{Q}_{\mathrm{T} 1}$ (Fig. 2.11) ${ }^{154}$.

There are two processes leading to a redistribution of populations at the $\mathbf{D}_{\mathbf{T 1}}$. The first one is the indirect process through ISC within the dye moiety $\left(\mathbf{S}_{\mathbf{1}} \rightarrow \mathbf{T}_{\mathbf{1}}\right)$ induced by fast spin-orbit interaction ${ }^{178}$. The second one is the direct process through the internal conversion (IC) $\mathbf{D}_{\mathbf{S} 1} \rightarrow \mathbf{D}_{\mathbf{T} 1}$. The $\mathbf{Q}_{\mathbf{T} 1}$ state can be populated only through the ISC. The indirect process converts rapidly the photoexcited $\mathbf{D}_{\mathbf{S} 1}$ state to the spin polarized $\mathbf{D}_{\mathbf{T} 1}$ and 


\section{Theoretical background}

$\mathbf{Q}_{\mathbf{T 1}}$ states. The direct process of the population transition from $\mathbf{D}_{\mathbf{S 1}}$ to $\mathbf{D}_{\mathbf{T 1}}$ is a result of mixing of $\mathbf{D}_{\mathbf{S} 1}$ and $\mathbf{D}_{\mathbf{T} 1}$, which is induced by the interaction of two electrons of the dye triplet compound with the electron of the radical doublet ${ }^{179,180}$. This transition is not spin selective ${ }^{180}$.

The transition from $\mathbf{D}_{\mathbf{T} \mathbf{1}}$ to $\mathbf{D}_{\mathbf{S} \mathbf{0}}\left(k_{D, D 0}\right)$ passes two routes with the conservation of a spin projection. The first is the IC and the second is the successive forward and backward electron transfer through charge transfer state. These two processes do not change the spin alignment ${ }^{181,182}$. The charge transfer (CT) was considered as the main way for triplet quenching of aromatic molecules by nitroxide radicals ${ }^{183,184}$. In the systems like fullerene-nitroxide, the electron transfer through $\mathrm{CT}$ state is more efficient than the direct IC from $\mathbf{D}_{\mathbf{T} 1}$ to $\mathbf{D}_{\mathbf{S} \mathbf{0}}{ }^{154}$. The rate of the transition from $\mathbf{Q}_{\mathbf{T} 1}$ to $\mathbf{D}_{\mathbf{S 0} 0}\left(k_{Q, D 0}\right)$ (Fig. 2.11) is much slower than $\mathrm{CT}$ at room temperature for the fullerene-nitroxide and thus is negligible in the population kinetics of the ground state $\mathbf{D}_{\mathbf{S} \mathbf{0}}{ }^{154}$.

Furthermore, the radiationless transition occurs between the doublet $\mathbf{D}_{\mathbf{T 1}}$ and the quartet $\mathbf{Q}_{\mathbf{T} 1}$ (Fig. 2.11). If the energy gap between $\mathbf{D}_{\mathrm{T} 1}$ and $\mathbf{Q}_{\mathbf{T} 1}$ is small $\left(3 J_{R T}\right.$ is much smaller than the vibrational quanta) and the vibrational relaxation within the $\mathbf{D}_{\mathbf{T 1}}$ state is very fast, the ISC between $\mathbf{D}_{\mathrm{T} 1}$ and $\mathbf{Q}_{\mathbf{T} 1}$ becomes reversible. This is in contrast to the case with a large energy gap, where a fast vibrational relaxation within the final $\mathbf{Q}_{\mathbf{T} 1}$ state makes the back ISC practically impossible. The reversible ISC between $\mathbf{D}_{\mathbf{T 1}}$ and $\mathbf{Q}_{\mathbf{T 1}}$ plays a key role in the reversed quartet mechanism (RQM). This back-ward ISC leads to the inversion of the electron spin polarization found in the fullerene-nitroxide derivatives (Chapter 6) ${ }^{154}$. The ratio of all allowed rates from $\mathbf{D}_{\mathbf{T} 1}$ to $\mathbf{Q}_{\mathbf{T} 1}\left(k_{D, Q}\right)$ and from $\mathbf{Q}_{\mathbf{T} 1}$ to $\mathbf{D}_{\mathbf{T} 1}$ $\left(k_{Q, D}\right)$ is 154,172

$$
\frac{k_{Q, D}\left(m_{D}, m_{Q}\right)}{k_{D, Q}\left(m_{D}, m_{Q}\right)}=\exp \left(-\frac{3 J_{R T}}{k T}\right) .
$$

The overall dynamics of the electron spin polarization of the radical-dye system in the presence of an external magnetic field includes electron and nuclear spin-lattice relaxations (Fig. 2.11). The probabilities of transitions inside the doublets $\mathbf{D}_{\mathbf{S} 0}$ and $\mathbf{D}_{\mathbf{T} 1}$ $\left(w_{D 0}\right.$ and $w_{D T}$, respectively) are approximately equal to each other whereas the transition 


\section{Theoretical background}

probabilities within the quartet $\mathbf{Q}_{\mathbf{T} 1}$ is higher than those of the doublets $\left(w_{Q T}>w_{D T} \approx w_{D 0}\right)$ because the spin-lattice relaxation within the quartet is induced by fluctuations of the zero field splitting (ZFS) interaction, which considerably exceeds the magnetic interactions in the doublet ${ }^{154}$. 


\section{Materials and methods}

\subsection{Investigated molecules}

A series of nitroxide derivatives functionalized with a fullerene $\mathrm{C}_{60}$ have been synthesized by Dr. Guoquan Liu (Fig. 3.1). Additional introduction of side chains into the fullerene cage increases the molecular size and the solubility in water (after removal of the protecting groups $-\mathrm{tBOC}){ }^{185}$. Despite of the solubility of FN-2a and FN-3a in water, the complete series of sample is soluble only in non-polar solvents such as toluene, which, partially, have the advantage of low dielectric losses for DNP experiments.

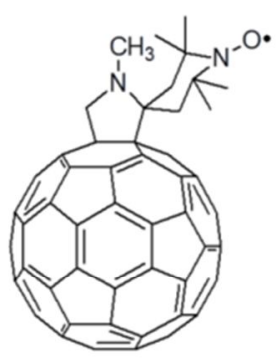

FN

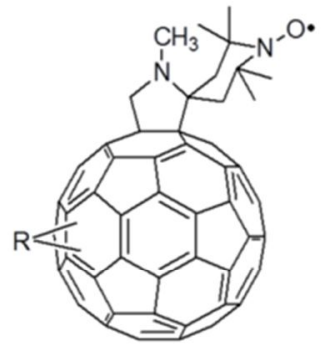

FN-1a

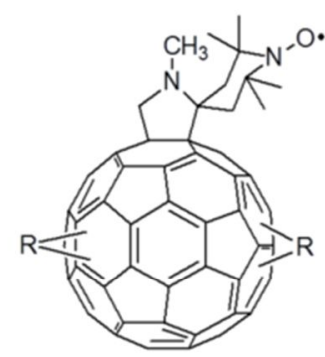

FN-2a

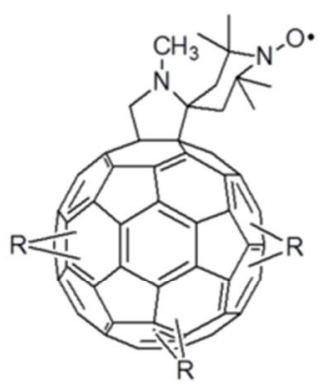

FN-3a<smiles>[R]C(=O)NCCCOC(=O)C=C(C=O)OCCCNC(=O)OCc1ccccc1</smiles>

Figure 3.1: Fullerene-nitroxide derivatives investigated in DNP experiments (red cycle: link position).

3.1.1. Synthesis. All reagents and solvents unless specified were purchased from Sigma-Aldrich and were used without further purification. NMR spectra were obtained on a Varian Mercury 300 spectrometer $\left(300 \mathrm{MHz}\right.$ for ${ }^{1} \mathrm{H}$ ). The synthesis procedure consists of two steps: the synthesis of precursor molecules (FN and malonate-derived compound) at first (Fig. 3.2) and then fullerene-nitroxide derivatives with the additional side chains (Fig. 3.3). 


\section{Materials and methods}

FN and the malonate-derived compound (1) were synthesized according to Scheme $\mathbf{1}$ following experimental procedures reported elsewhere 160, 185 1 ESI-MS: $419.2 \mathrm{~m} / \mathrm{z}$ $[\mathrm{M}+\mathrm{H}]^{+},{ }^{1} \mathrm{H}$ NMR $\left(300 \mathrm{MHz}, \mathrm{CDCl}_{3}\right) \delta / \mathrm{ppm} 4.84$ (s, 2H), 4.21 (t, 4H), 3.39 (s, 2H), $3.19(\mathrm{t}, 4 \mathrm{H}), 1.84(\mathrm{~m}, 4 \mathrm{H}), 1.43(\mathrm{~s}, 18 \mathrm{H})$. FN (MALDI-MS: $917.2 \mathrm{~m} / \mathrm{z}[\mathrm{M}]^{-}$, UV-vis (toluene): $434 \mathrm{~nm}$ ).

a)
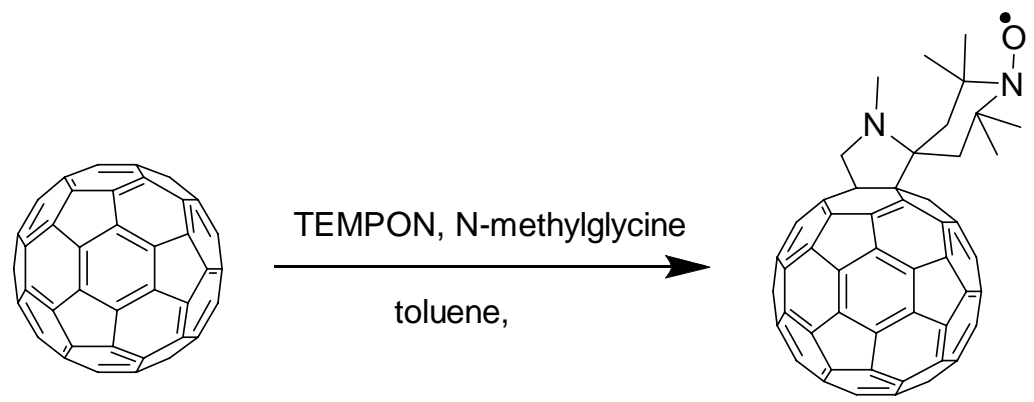

FN

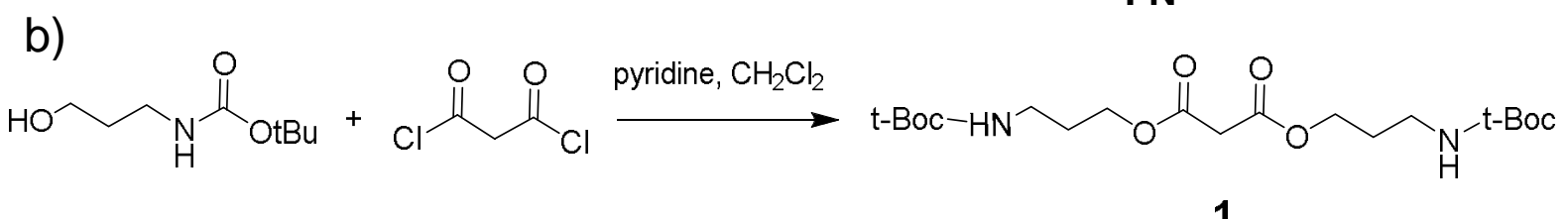

Figure 3.2: Scheme of the synthesis of precursors $\mathbf{F N}$ (a) and $\mathbf{1}$ (b).

FN-1a, FN-2a and FN-3a were synthesized by adapting a previous report ${ }^{185}$, in which pristine $\mathrm{C}_{60}$ instead of $\mathbf{F N}$ was used as building block for the synthesis of water-soluble fullerene derivatives. A modified Bingel reaction that uses mild conditions $(1,8-$ diazabicyclo[5.4.0] undec-7-ene and $\mathbf{C B r}_{4}$ as bromine source) for the in situ formation of $\alpha$-bromomalonates was selected here to preserve the nitroxide functionality. $\mathbf{F N}$ and $\mathbf{1}$ were reacted in toluene for 30-60 $\mathrm{min}$ in the presence of $\mathbf{C B r}_{\mathbf{4}}$, and 1,8diazabicyclo[5.4.0] undec-7-ene (DBU) to obtain the target fullerene adducts using the conditions shown in Table 3.1. The desired products were purified by column chromatography (silica gel) using toluene/ethyl acetate as eluent with a yield of c.a. $40 \%$ for FN-1a (MALDI-MS: $1333.3 \mathrm{~m} / \mathrm{z}$ [M] ${ }^{-}$, UV-vis (toluene): $480 \mathrm{~nm}$ ), c.a. $20 \%$ for FN-2a (MALDI-MS: $1749.2 \mathrm{~m} / z$ [M] $]^{-}$, UV-vis (toluene): $472 \mathrm{~nm}$ ) and c.a. $8 \%$ for $\mathbf{F N - 3 a}$ (MALDI-MS: $2165.2 \mathrm{~m} / \mathrm{z}[\mathrm{M}]^{-}$, UV-vis (toluene): $460 \mathrm{~nm}$ ). 


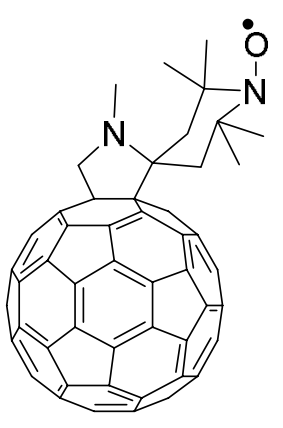

FN

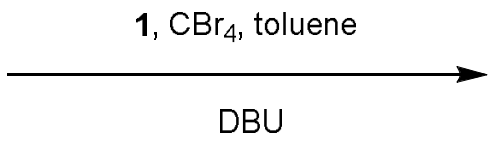

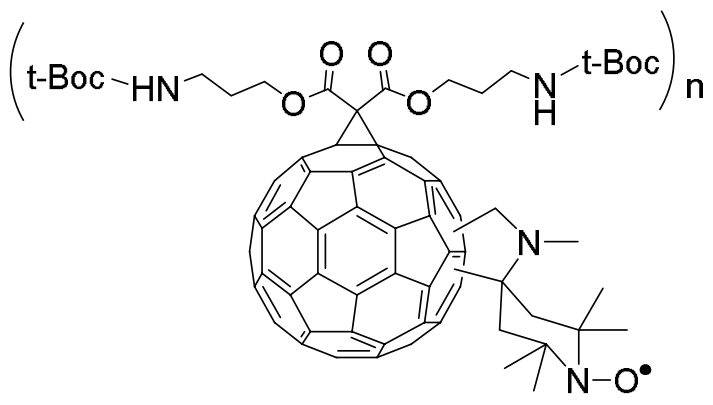

FN-1a $n=1$

FN-3a $n=3$

Figure 3.3: Scheme of the synthesis of fullerene-nitroxide derivatives by nucleophilic addition of the $\alpha$-bromomalonate carbanion derivated to FN (Bingel-type reaction).

Table 3.1: Ratio of reactants in the synthesis of fullerene-nitroxide derivatives.

\begin{tabular}{c|c|c|c|c}
\hline Sample & $\begin{array}{c}\text { FN } \\
\text { (equiv) }\end{array}$ & $\begin{array}{c}\mathbf{C B r}_{\mathbf{4}} \\
\text { (equiv) }\end{array}$ & $\begin{array}{c}\mathbf{1} \\
\text { (equiv) }\end{array}$ & $\begin{array}{c}\text { DBU } \\
\text { (equiv) }\end{array}$ \\
\hline FN-1a & 1 & 1.5 & 1.5 & 3 \\
\hline FN-2a & 1 & 2.2 & 2.2 & 4.5 \\
\hline FN-3a & 1 & 3.3 & 3.3 & 6.6 \\
\hline
\end{tabular}

Mass spectrometry was carried out on a Bruker Ultraflex III MALDI-TOF spectrometer using DCTB as matrix $(355 \mathrm{~nm})$ or on a Bruker MicroTOF with electrospray ionization (ESI). UV-vis spectra were recorded at room temperature in quartz cuvettes on an Agilent Cary 100 UV-VIS spectrophotometer (Fig. 3.4). 


\section{Materials and methods}

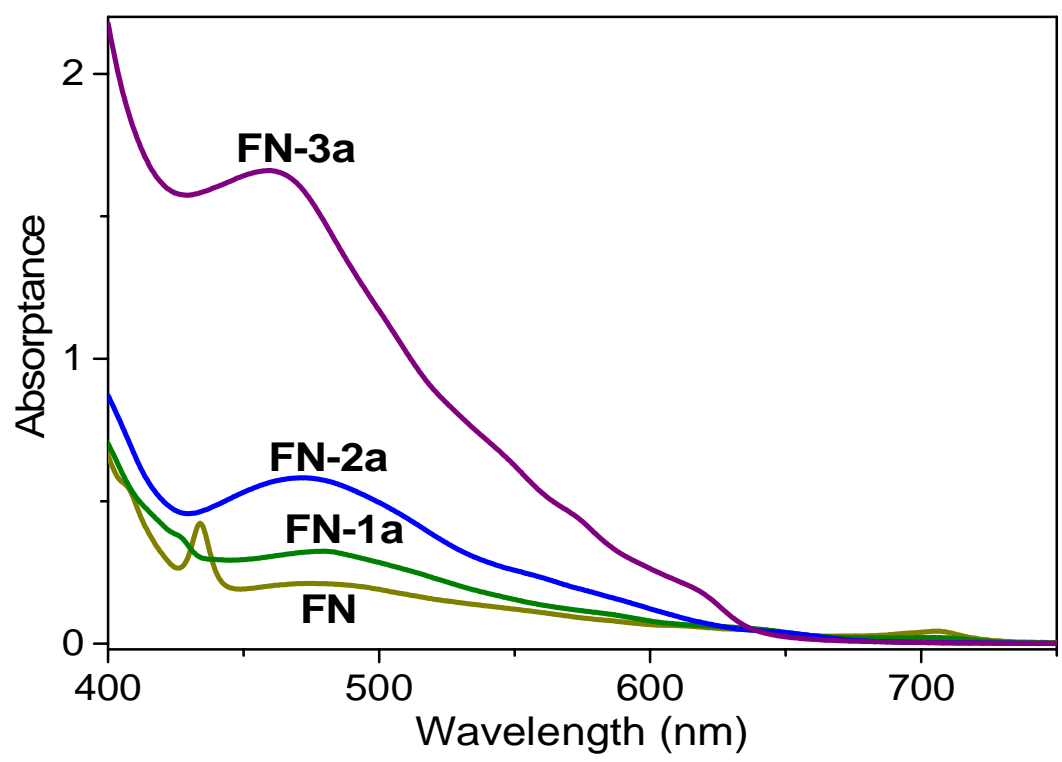

Figure 3.4: UV-Vis absorption spectra of fullerene-nitroxide derivatives $(\sim 0.1 \mathrm{mM})$ in toluene at room temperature.

\subsection{Rotational correlation time}

The rotational diffusional motion is a random process. The characteristic parameter of this process is rotational correlation time $\left(\tau_{c}\right)$ which denotes the time of molecular orientation alignment loss. The rotational motion leads to fluctuation local magnetic field and modulations of the resonance frequencies for electron spin resonance (ESR) transition. It is reason why ESR spectroscopy can observe the dynamic of tumbling of molecules in liquid solutions ${ }^{186}$.

3.2.1. Fast-motion regime. In the fast-motion regime, where the speed of rotational diffusion $\left(\tau_{c}^{-1}\right)$ is fast as compared to the anisotropic interactions, the Redfield theory leads to simplified expressions for EPR line widths in solution. For the case of an EPR line with hyperfine and $g$ anisotropy, the widths of individual hyperfine lines are given by the quadratic expression ${ }^{186}$ :

$$
\Delta v=A_{0}+A+B \cdot m_{I}+C \cdot m_{I}^{2},
$$




\section{Materials and methods}

where $\Delta v$ is the linewidth of the Lorentzian line in frequency units, $m_{I}$ is the nuclear magnetic quantum number of the considered hyperfine line, $(A, B, C)$ are coefficients that characterize the averaging of the anisotropy by molecular motion. $\mathrm{A}_{0}$ includes all other broadening effects. With knowledge of the $\boldsymbol{g}$ and $\boldsymbol{A}$ (hyperfine) tensors, the isotropic rotational correlation time $\tau_{c}$ can be obtained from $A, B, C$ according to ${ }^{186}$ :

$$
\begin{gathered}
A=\frac{\mathrm{m}_{e}^{2}}{h^{2}} B_{0}^{2}(\mathrm{D} g: \mathrm{D} g)\left[\frac{2}{15} j_{0}+\frac{1}{10} j_{1}\right]+I(I+1)(\mathrm{D} A: \mathrm{D} A)\left[\frac{2}{15} j_{0}+\frac{7}{60} j_{1}\right] \\
B=\frac{16 \mathrm{p}^{2}}{15} \frac{\mathrm{m}_{e}}{h} B_{0}(\mathrm{D} g: \mathrm{D} A)\left[\frac{5}{15} j_{0}+\frac{1}{5} j_{1}\right] \\
C=(\mathrm{D} A: \mathrm{D} A)\left[\frac{1}{12} j_{0}-\frac{1}{60} j_{1}\right]
\end{gathered}
$$

with spectral densities of the form $j_{0}=\tau_{C}$ and $j_{1}=\tau_{C} /\left(1+\omega_{0}^{2} \tau_{C}^{2}\right) . \Delta g$ and $\Delta \boldsymbol{A}$ are the anisotropic parts of the $\boldsymbol{g}$ and $\boldsymbol{A}$ tensors, i.e. $\boldsymbol{\Delta g}=\boldsymbol{g}-g_{\text {iso }} \mathbf{1}$ and $\Delta \boldsymbol{A}=\boldsymbol{A}-a_{\text {iso }} \mathbf{1}(\boldsymbol{\Delta A}$ is given in frequency units, $\mathbf{1}$ is the unit matrix). The notation $X: Y$ indicates element-byelement multiplication of the matrices followed by summation, giving a number characterizing the corresponding anisotropy.

\subsection{Liquid-state DNP at $0.35 \mathrm{~T}$}

3.3.1. Experimental setup for DNP. The low-field DNP spectrometer consists of a commercial Bruker ElexSys E500 CW EPR spectrometer equipped with a Bruker $5 \mathrm{~W}$ CW amplifier (AmpX) and a Bruker Minispec for NMR detection (2-65 MHz). A Bruker FlexLine dielectric microwave resonator (EN 4118X-MD-4) with matched ENDOR coils for proton NMR at $14 \mathrm{MHz}$ is used for X-band $(9.7 \mathrm{GHz})$ EPR and DNP. For DNP, a liquid sample is continuously irradiated for several seconds by microwave in resonance with one of the hyperfine EPR transitions and subsequently the nuclear magnetization is measured with FID. To avoid heating of the resonator, a constant flow of $\mathrm{N}_{2}$ gas inside of the cavity is supplied.

The samples, containing $20 \mu \mathrm{L}$ of toluene solution doped with nitroxides, were degassed by three freeze-pump-thaw cycles whereas the water solution samples of $0.6 \mu \mathrm{L}$ 


\section{Materials and methods}

were non-degassed. In the DNP experiments for the toluene solution, the irradiation time was set to be $2-30 \mathrm{~s}$ (depending on the concentration) at MW power $\approx 3 \mathrm{~W}$ (which corresponds to MW field strength $B_{1} \approx 3 \mathrm{G}$ ) and 1 or 8 scans for DNP measurements and 128 scans for Boltzmann measurements. The experiments with water solutions were done with the same MW power but using 1-5 s of MW irradiation duration. 64 and 1024 scans were applied to DNP and Boltzmann measurements, respectively.

3.3.2. DNP enhancement measurement. The different enhancements of two types of protons of toluene (ring and methyl) were obtained as following. Firstly, the amplitude ratios of DNP vs Boltzmann signal were calculated for the two individual peaks in the spectra and related to the DNP enhancement $\varepsilon$ according to:

$$
\begin{aligned}
& \varepsilon_{\text {Ring }}=\frac{I_{\text {Ring }, D N P}}{I_{\text {Ring }, \text { Bol }}} \approx \frac{\Delta v_{\text {Ring }, D N P} \cdot h_{\text {Ring }, D N P}}{\Delta v_{\text {Ring }, \text { Bol }} \cdot h_{\text {Ring, DNP }}}=k_{\text {Ring }} \cdot E_{\text {Ring }} \\
& \varepsilon_{\text {Methyl }}=\frac{I_{\text {Methyl, DNP }}}{I_{\text {Methyl, Bol }}} \approx \frac{\Delta v_{\text {Methyl, DNP }} \cdot h_{\text {Methyl, DNP }}}{\Delta v_{\text {Methyl, Bol }} \cdot h_{\text {Methyl, DNP }}}=k_{\text {Methyl }} \cdot E_{\text {Methyl }},
\end{aligned}
$$

where $I$ is the area of the NMR Lorentzian line given by $I=1.57 \cdot h \cdot \Delta v_{1 / 2}{ }^{70}, h$ is the maximum of the absorption line, $\Delta v$ is the width at half height, $E=h_{D N P} / h_{B o l}$ or the amplitude ratios. The factor $k=\Delta v_{D N P} / \Delta v_{B o l}$ is introduced to account for the artificial broadening observed after signal averaging. This broadening is due to field instability caused by the electromagnet (no shimming is available in our set up). Assuming that line broadening is similar after same number of accumulations, we set $k_{\text {ring }} \approx k_{\text {methyl }} \approx k$ and therefore $\varepsilon_{\text {ring }}=k \cdot E_{\text {ring }}, \varepsilon_{\text {methyl }}=k \cdot E_{\text {methyl }}$. Subsequently the enhancement is calibrated with the first point of the FID, for which the line broadening effect can be neglected. The first point of the FID reflects the DNP signal enhancement $\varepsilon_{F I D}$ according to:

$$
\frac{5 \cdot \varepsilon_{\text {Ring }}+3 \cdot \varepsilon_{\text {Methyl }}}{8}=\varepsilon_{F I D},
$$

In this expression, the Boltzmann FID signal is normalized to unity with an intensity ratio of the two proton groups (ring : methyl) of 5:3. By substituting $\varepsilon_{\text {ring }}=k \cdot E_{\text {ring }}$, $\varepsilon_{\text {methyl }}=k \cdot E_{\text {methyl }}$ in the equation (3.4) we obtain:

$$
\frac{k \cdot\left(5 \cdot E_{\text {Ring }}+3 \cdot E_{\text {Methyl }}\right)}{8}=\varepsilon_{F I D},
$$




\section{Materials and methods}

From the observed FID enhancement and the enhancement of the amplitudes $E_{\text {ring }}$ and $E_{\text {methyl }}$ we can calculate with the equation (3.5) the factor $k$, which is then inserted in the equation (3.3) to obtain the absolute enhancement $\varepsilon_{\text {ring }}$ and $\varepsilon_{\text {methyl }}$ of each type of protons.

\subsection{Electron-electron double resonance (ELDOR)}

In an electron-electron double resonance (ELDOR) experiment the first frequency is used for pumping one of possible EPR transitions whereas the second one detects the signal behaviour (reduction) of any other transition. The ELDOR spectrum displays a dependence of signal intensity on the pumping frequencies ${ }^{187}$.

It is demanding to pump all three nitroxide lines simultaneously due to a large frequency separation between hf transitions. Nevertheless, as a result of interactions between different hyperfine transitions (e.g. nuclear relaxation and HE; Fig. 2.2), the "not irradiated" EPR lines can be saturated as well. ELDOR experiment is an appropriate method to estimate the total saturation of a complete paramagnetic system ${ }^{73}$, 163. ELDOR can record the FID signal of one EPR transition, which corresponds to a fixed detecting frequency, at various pumping frequency values (Fig. 3.5). The signal reductions appear at frequencies corresponding to one of the EPR transitions. 


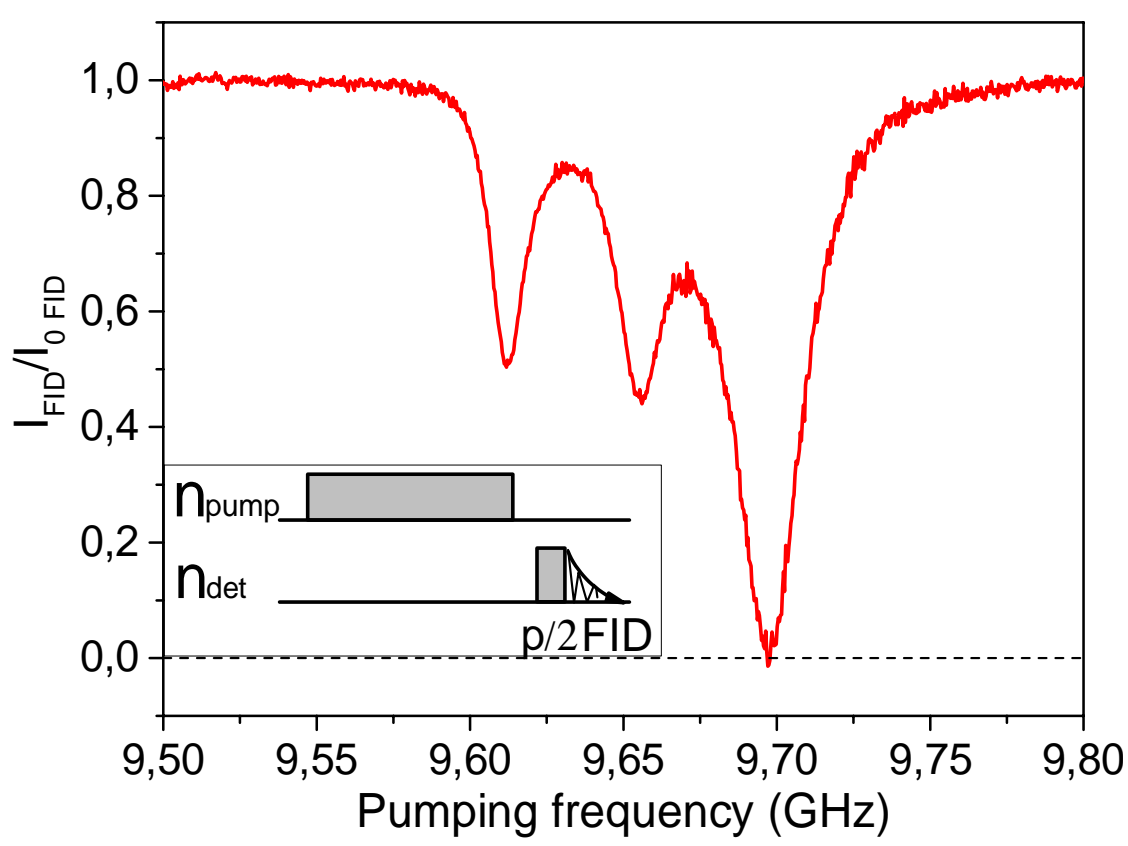

Figure 3.5: Normalized spectrum of an ELDOR experiment with TEMPOL radical in toluene solution. Here three signal reductions give the saturation factor values of the low field EPR line in the case of the MW irradiation of the low field (right), central (middle) and high field (left) EPR transitions. Insert: the scheme of the pulse sequence ${ }^{163}$.

Another type of ELDOR experiment, which is performed while varying the time delay between the pump and detection pulses, allows for monitoring the EPR polarization recovery dynamics (Fig. 3.6). As discussed in Chapter 2, they enable to determine all rate parameters describing the interaction between hyperfine sublevels by means of a concurrent (simultaneous) fitting of the recovery curves $73,163,164,166$. 


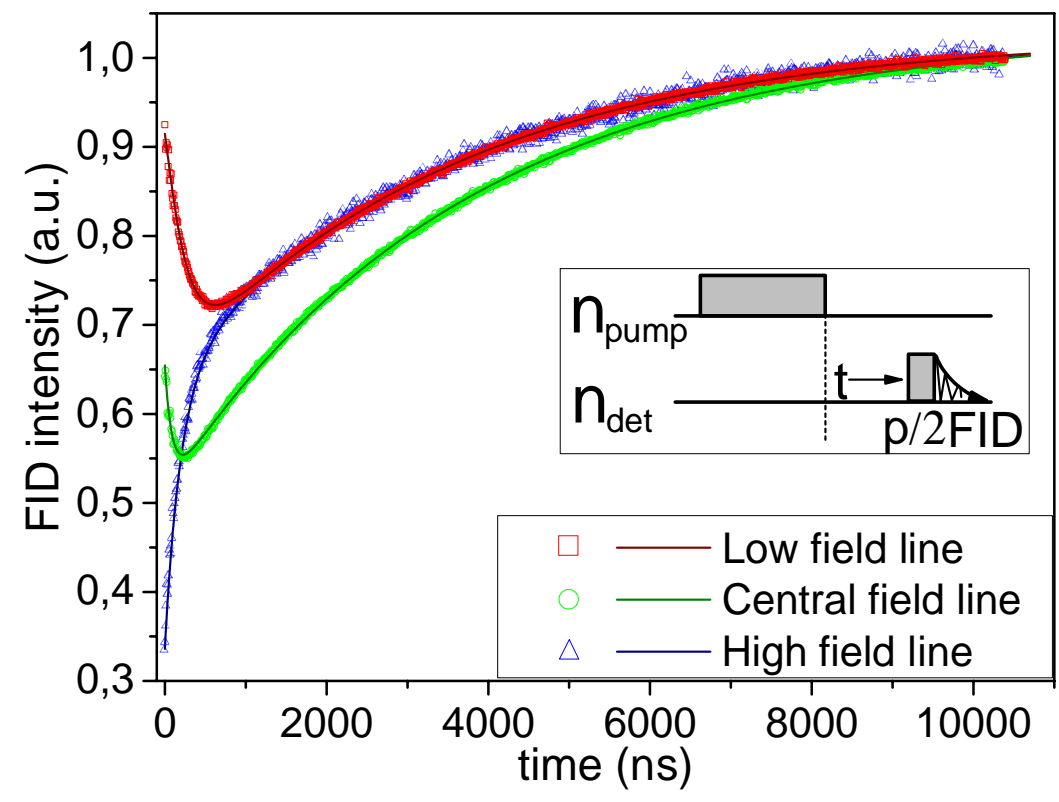

Figure 3.6: Saturation recovery curves of FN-2a (Chapter 5) for pumping high field EPR transition. Insert: the scheme of the pulse sequence ${ }^{163}$.

\subsection{Transient EPR (TrEPR)}

Time-resolved/transient electron paramagnetic resonance (TrEPR) spectroscopy is a well-established experimental technique to monitor the time evolution of the electron spin polarization ${ }^{188}$. TrEPR is widely used in material science and biochemistry, particularly photochemistry, to detect short-lived transient radicals ${ }^{189-191}$ and ions ${ }^{191-195}$ or to observe electron spin transfer ${ }^{196,197}$ and generation of multiplet spin states (triplet, quartet) ${ }^{160-162,174,198-200}$. The time resolution can reach tens nanoseconds. In a common CW EPR, it is impossible to change the magnetic field sufficiently rapidly to sweep through the spectrum on the submicrosecond timescale. The TREPR has been developed to overcome this problem and to obtain the spectrum as soon as possible after appearance of a paramagnetic species. The first TrEPR experiments were performed in 1960s and 1970s to study radicals and ions in solutions by Atkins et al ${ }^{201,202}$, radiolysis by Smaller et al ${ }^{190}$, photolysis ${ }^{203}$ and chemical induced dynamic nuclear polarization (CIDEP) ${ }^{202 \text {, }}$ 204, 205 


\section{Materials and methods}

3.5.1. Setup for CW TrEPR with photoexcitation. The setup to detect fast changes of spin polarization consists of a commercial Bruker ElexSys E500 CW EPR spectrometer at $0.35 \mathrm{~T}$ and a Bruker FlexLine dielectric microwave resonator (EN 4118X-MD-4). Photoexcitation was performed with Continuum Nd-YAG pulsed laser (Sureliet I $20 \mathrm{~Hz}$ repetition rate, pulse length $\sim 4-5 \mathrm{~ns}$, 2nd harmonic $532 \mathrm{~nm}$ ). For transient EPR a liquid sample is irradiated by a short high power laser pulse $(\sim 5 \mathrm{~mJ}$ per pulse) and subsequently the time evolution of EPR signal at a correspondent magnetic field value is detected. A LeCroy oscilloscope (WaveJet 354, $500 \mathrm{MHz}$ ) and a Philips pulse generator (PM 5786) were used to synchronize the EPR signal detection with laser pulse excitation. Samples with $20 \mu \mathrm{L}$ toluene solution (concentration $\sim 1.5 \mathrm{mM}$ ) were degassed by three freeze-pump-thaw cycles. To avoid heating of the resonator and samples, a constant flow of $\mathrm{N}_{2}$ gas inside of the cavity was supplied. The same optical setup was used for detection of time evolution of the excited system by pulsed EPR.

3.5.2. Continuous wave transient EPR (CW TrEPR). At the initial condition, the electron spins are precessing around the applied magnetic field, thus, making up the initial magnetization (i.e. polarization) $M_{z}(0)$, which is aligned along the field $(z$ is direction of the magnetic field) (Fig. 3.7). $M_{z}(t)$ defines the time evolution of the magnetization along axis $z$ of the detected short-lived radical. Under a microwave irradiation, which is in resonance with Zeeman splittings at a given field and is aligned along the axis $x$ (laboratory frame), the magnetization vector is rotated, thus, transforming into $M_{y} \neq 0$ component in the laboratory frame. This component $\left(M_{y}(t)\right)$ varied with the time shows up as the transient EPR signal ${ }^{206}$. 


\section{Materials and methods}
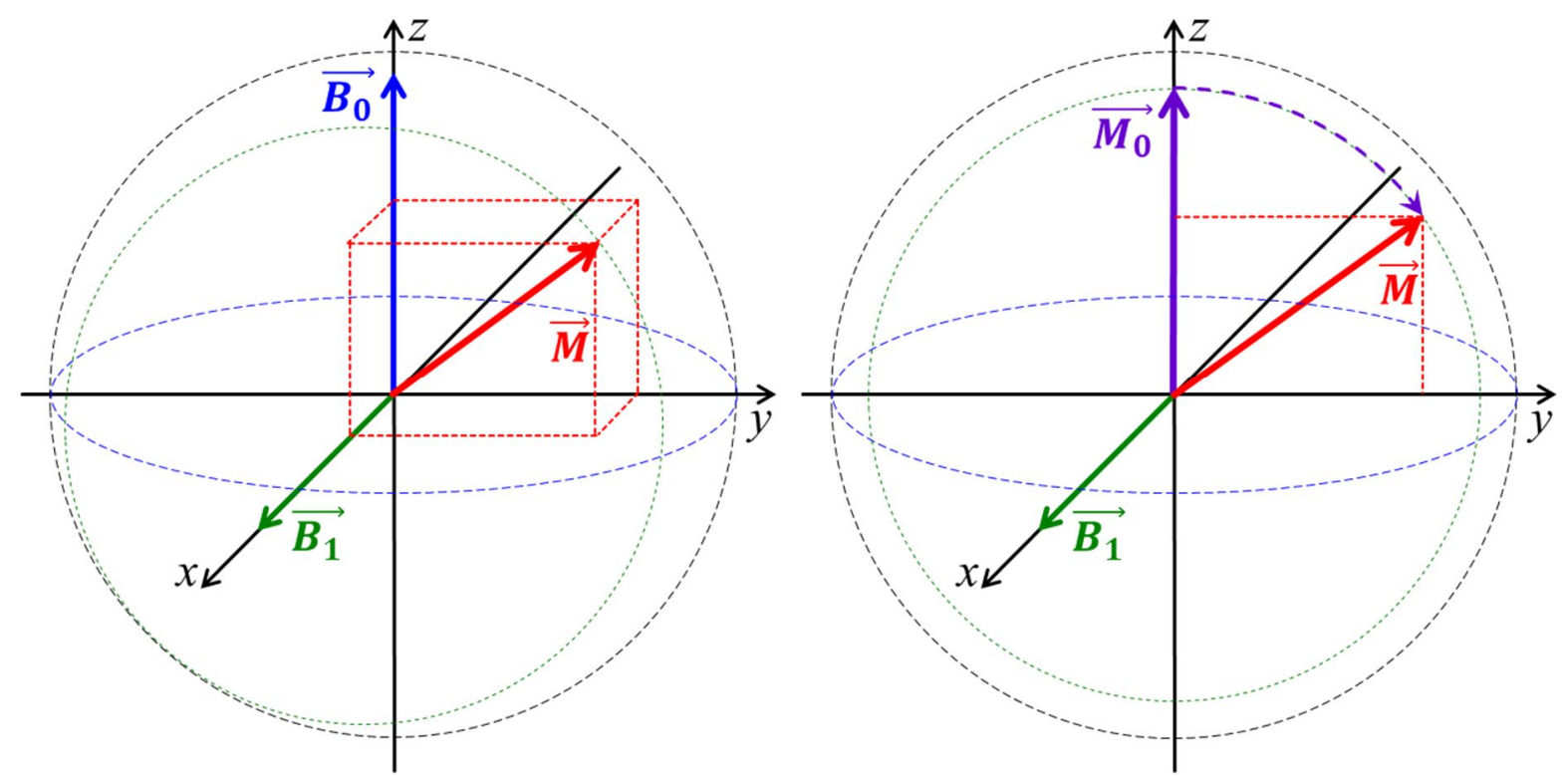

Figure 3.7: Scheme of the magnetization vector behaviour $\stackrel{r}{M}(t) . \stackrel{r}{B_{0}}$ is an external applied magnetic field; $\stackrel{r}{B_{1}}$ is a microwave field strength. Left: general case (off resonance); right: on resonance with initial condition $\stackrel{r}{M_{0}}$.

The magnetization vector of two-level spin system $\stackrel{r}{M}$ has a $z$-component proportional to the population difference $\left(n_{1}-n_{2}\right)$. So, this vector can be considered as a product of the total population $\left(n_{1}+n_{2}\right)$ and the "polarization" vector $\left.\stackrel{r}{\mu} \stackrel{r}{r}=n \cdot \stackrel{r}{\mu}\right)$ with its $z$-component proportional to the spin polarization $P=\frac{n_{1}-n_{2}}{n_{1}+n_{2}}$ of the observed system. The polarization vector $(\stackrel{r}{\mu})$ does not depend on the kinetics of the radical, and only includes rotation and relaxation effects. The dynamics of TrEPR signal can be described by Bloch equation ${ }^{207}$ :

$$
\frac{d}{d t} \stackrel{r}{\mu}=\left(\begin{array}{ccc}
-T_{2}^{-1} & \Delta \omega & 0 \\
-\Delta \omega & -T_{2}^{-1} & \omega_{1} \\
0 & -\omega_{1} & -T_{1}^{-1}
\end{array}\right)\left(\begin{array}{c}
\mu_{x} \\
\mu_{y} \\
\mu_{z}-P_{e q}
\end{array}\right), r_{0}=\left(\begin{array}{c}
0 \\
0 \\
P_{0}
\end{array}\right)
$$

where $T_{1}$ and $T_{2}$ are spin-lattice and spin-spin relaxation times, respectively, $\Delta \omega$ is an offset from the resonance frequency, $\omega_{1}$ is the microwave field strength, $P_{e q}, P_{0}$ are thermal equilibrium and initial spin polarizations, respectively. The equation (3.6), if including chemical exchange (Bloch-McConnel equation) ${ }^{208-210}$, is: 


\section{Materials and methods}

$$
\begin{gathered}
\frac{d}{d t} \stackrel{r}{M}=n \cdot \frac{d}{d t} \underset{\mu}{\mu}+\frac{d}{d t} n \cdot \stackrel{r}{\mu} \\
\frac{d}{d t}\left(\begin{array}{l}
M_{x} \\
M_{y} \\
M_{z}
\end{array}\right)=\left(\begin{array}{ccc}
-T_{2}^{-1} & \Delta \omega & 0 \\
-\Delta \omega & -T_{2}^{-1} & \omega_{1} \\
0 & -\omega_{1} & -T_{1}^{-1}
\end{array}\right)\left(\begin{array}{c}
M_{x} \\
M_{y} \\
M_{z}-n \cdot P_{e q}
\end{array}\right)+\frac{r \&}{n} \cdot\left(\begin{array}{l}
M_{x} \\
M_{y} \\
M_{z}
\end{array}\right),\left(\begin{array}{l}
M_{x 0} \\
M_{y 0} \\
M_{z 0}
\end{array}\right)=n(0) \cdot\left(\begin{array}{c}
0 \\
0 \\
P_{0}
\end{array}\right) .
\end{gathered}
$$

The equation (3.7) has a general analytical solution ${ }^{206,211}$, which is:

$$
\left(\begin{array}{l}
M_{x} \\
M_{y} \\
M_{z}
\end{array}\right)=n(t) \cdot\left\{e^{\Omega t} \cdot\left(P_{0} E+P_{e q} \frac{1}{T_{1}} \Omega^{-1}\right)-P_{e q} \frac{1}{T_{1}} \Omega^{-1}\right\} \cdot\left(\begin{array}{l}
0 \\
0 \\
1
\end{array}\right), \Omega=\left(\begin{array}{ccc}
-T_{2}^{-1} & \Delta \omega & 0 \\
-\Delta \omega & -T_{2}^{-1} & \omega_{1} \\
0 & -\omega_{1} & -T_{1}^{-1}
\end{array}\right),
$$

where $\Omega^{-1}$ and $e^{\Omega t}$ represent the matrix inversion and the matrix exponent, respectively, $E$ is the identity matrix. A detailed numerical analysis of the time-dependent Bloch equation including chemical exchange has been done by Murase and et al ${ }^{212,213}$.

3.5.3. Time evolution of EPR signal by pulsed EPR. With a short microwave pulse the magnetization vector $\dot{M}$ of electron spins at a constant magnetic field can be rotated (e.g. from axis $z$ into the $x-y$ plane). If the applied microwave pulse rotates $\dot{M}$ by $\pi / 2$ radian $\left(90^{\circ}\right)$, it is called “ $\pi / 2$ "-pulse. The subsequent behaviour of $x$ - and $y$-components of the magnetization is called free induction decay (FID). If FID can be recovered with the so-called "Hahn-echo" pulse sequence, the "spin echo" phenomenon can be observed. EPR spectroscopy, which is based on FID- and echo-detection is called pulsed EPR ${ }^{187}$. If microwave field strength is aligned along the $x$-axis, the tipped magnetization vector is aligned along the $y$-axis (Fig. 3.7) and the general solution of the Bloch equation (3.7) is:

$$
\left(\begin{array}{l}
M_{x} \\
M_{y} \\
M_{z}
\end{array}\right)=n(t) \cdot\left\{e^{\Omega t} \cdot\left(P_{0}\left(\begin{array}{l}
0 \\
1 \\
0
\end{array}\right)+P_{e q} \frac{1}{T_{1}} \Omega^{-1}\left(\begin{array}{l}
0 \\
0 \\
1
\end{array}\right)\right)-P_{e q} \frac{1}{T_{1}} \Omega^{-1}\left(\begin{array}{l}
0 \\
0 \\
1
\end{array}\right)\right\} .
$$

Pulsed EPR can be applied to detect behaviour of short-lived radicals also in the time range exceeding their transverse relaxation (from $\mu$ s to ms range). By changing the delay 


\section{Materials and methods}

between the light excitation and the applied " $\pi / 2$ " microwave pulse, the time evolution of the FID or echo signal from a studied system can be recorded (Fig. 3.8).

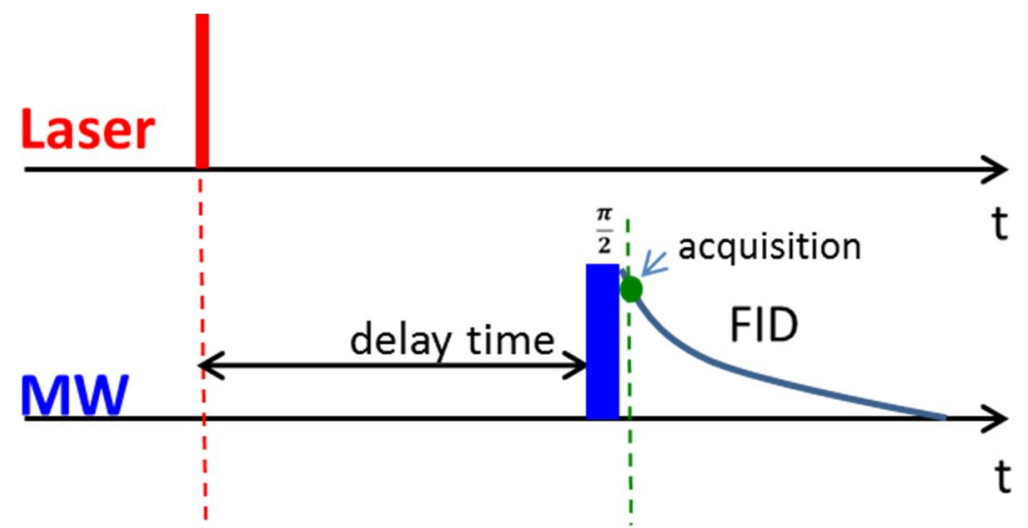

Figure 3.8: Pulse sequence of the pulsed EPR experiment with photoexcitation. Laser is used as an external source for the optical excitation to create excited transient (triplet) states or transient radicals as in the case of photolysis.

Unlike CW-TREPR, pulsed EPR allows to detect signals of both the steady state thermal equilibrium and short-lived excited states of the radical. This makes it possible to evaluate the important parameters of the observed system like spin polarization (enhancement) or amount of the excited molecules. Disadvantage of the pulsed EPR is the so-called "dead" time between the " $\pi / 2$ "-pulse and the acquisition starting point. It does not allow detecting signals from a system with the short spin-spin relaxation time $\left(T_{2}\right)^{187}$. 


\section{Comparative DNP studies in water and toluene: effect of solvent}

Unlike water, as the main solvent for applications in biological systems ${ }^{77,214}$, the nonpolar solvents, such as toluene, benzene, hexane, ${ }^{100,215,216}$ are also recently becoming attractive to host more complex organic polarizers. Moreover, the non-polar solvents have low dielectric losses, resulting in low sample heating upon microwave irradiation and thus allow working with sample volume close to that used for NMR experiments. Therefore, we have analysed DNP experiments with TEMPONE-D $16^{-}{ }^{15} \mathrm{~N}$ (4-OxoTEMPO) in toluene solution.

\subsection{EPR of TEMPONE-D $16^{15} \mathrm{~N}$ in toluene solution at $0.3 \mathrm{~T}$}

CW EPR spectra of TEMPONE-D ${ }_{16^{-}}{ }^{15} \mathrm{~N}$ in toluene solution showed two lines due to hyperfine interaction of an unpaired electron with ${ }^{15} \mathrm{~N}$ nucleus (Fig. 4.1a). As expected, the intensities of the EPR spectra increased with higher concentration, but at $5 \mathrm{mM}$ a significant broadened line shape was observed. The power dependence of the EPR signal intensity demonstrates that the saturation by MW reaches the maximal value for all investigated concentrations at $P_{M W}=3 \mathrm{~W}$, which is the power used in our DNP experiments (Fig. 4.1b). 


\section{Comparative DNP studies in water and toluene: effect of solvent}

a)

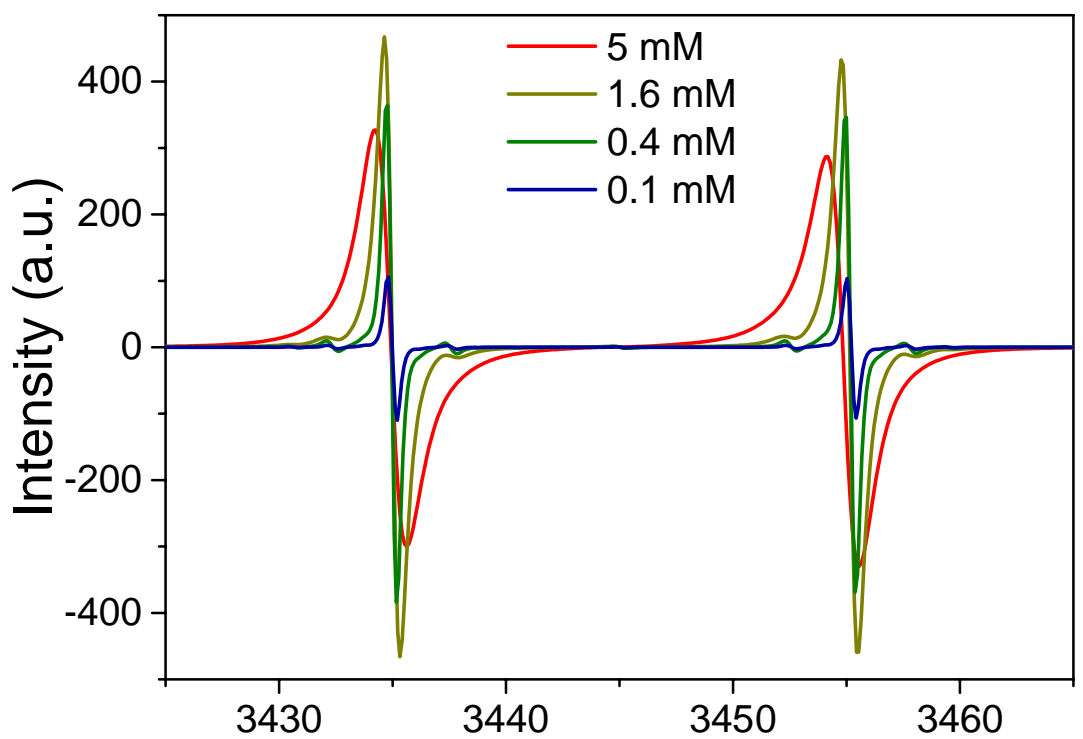

Field $(\mathrm{G})$

b)

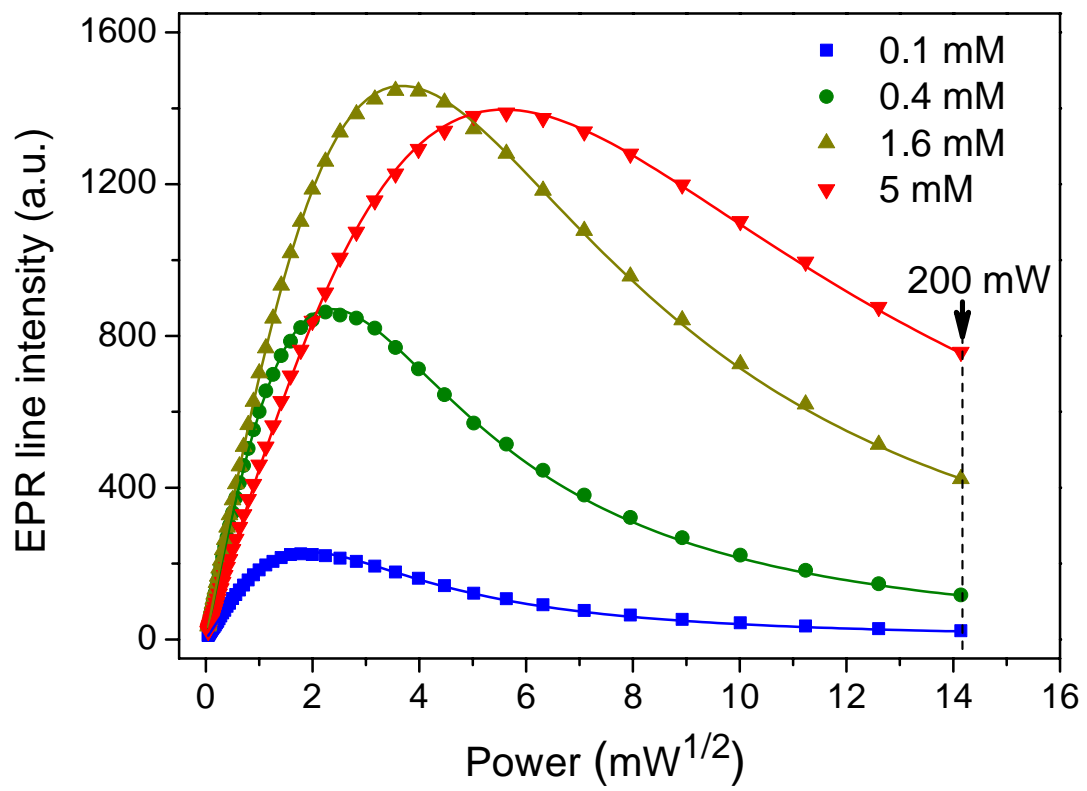

Figure 4.1: (a) CW-EPR spectra of TEMPONE- $\mathrm{D}_{16^{-}}{ }^{15} \mathrm{~N}$ in toluene at different concentrations at MW power $2 \mathrm{~mW}$. Experimental parameters: $1 \mathrm{G}$ modulation amplitude, $100 \mathrm{kHz}$ modulation frequency, 5.12 ms conversion time, 1024 points. (b) Peak-to-trough intensity power dependence of the low field EPR line (the derivative of Lorentzian line) for TEMPONE-D $16^{-}{ }^{15} \mathrm{~N}$ in toluene at different concentrations and fitting curves $(2.12)^{70}$.

\subsection{DNP with TEMPONE- $\mathrm{D}_{16^{-}}{ }^{15} \mathrm{~N}$ in toluene solution at $0.3 \mathrm{~T}$}

4.2.1. Enhancement. Two kinds of protons in toluene (the ring and methyl) were distinguished in the DNP experiment at $0.35 \mathrm{~T}$ (Fig. 4.2a). Two peaks are resolved in the 


\section{Comparative DNP studies in water and toluene: effect of solvent}

NMR spectra with a separation about $70 \mathrm{~Hz}$, which corresponds to $5 \mathrm{ppm}$. In result of MW irradiation on-resonant low-field EPR transition of the radical, the detected NMR transition has been enhanced by more than two orders of magnitude. Similar DNP enhancements in other solvents required much higher radical concentration $(20-100 \mathrm{mM})$ than in toluene case $(\leq 5 \mathrm{mM})$. The maximum achieved DNP enhancement $\varepsilon_{\text {ring }}$ and $\varepsilon_{\text {methyl }}$ are -159 and -137 , respectively, with errors around $\pm 5 \%$ (Fig. 4.2a). Comparing with DNP in water solution at the same experimental conditions it has been found that DNP enhancements in toluene are significantly higher at low radical concentration $(\leq 5 \mathrm{mM})$ but the maximum value are slightly lower at high concentrations (Fig. 4.2b). 


\section{Comparative DNP studies in water and toluene: effect of solvent}

a)

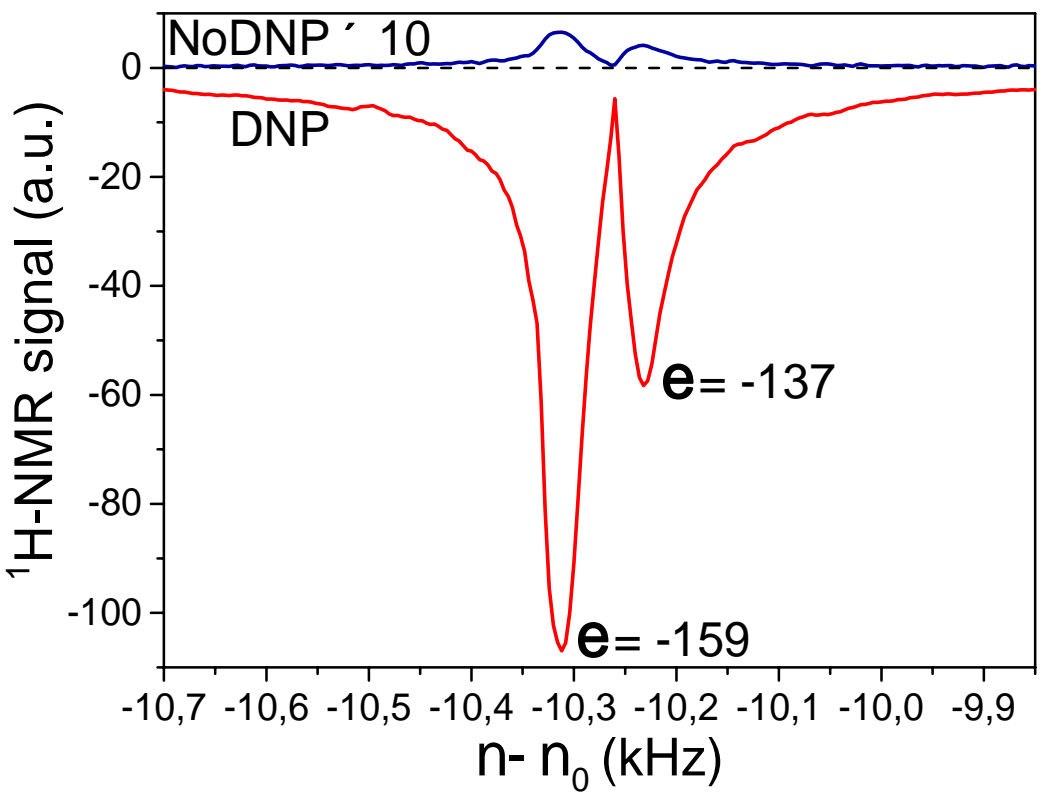

b)

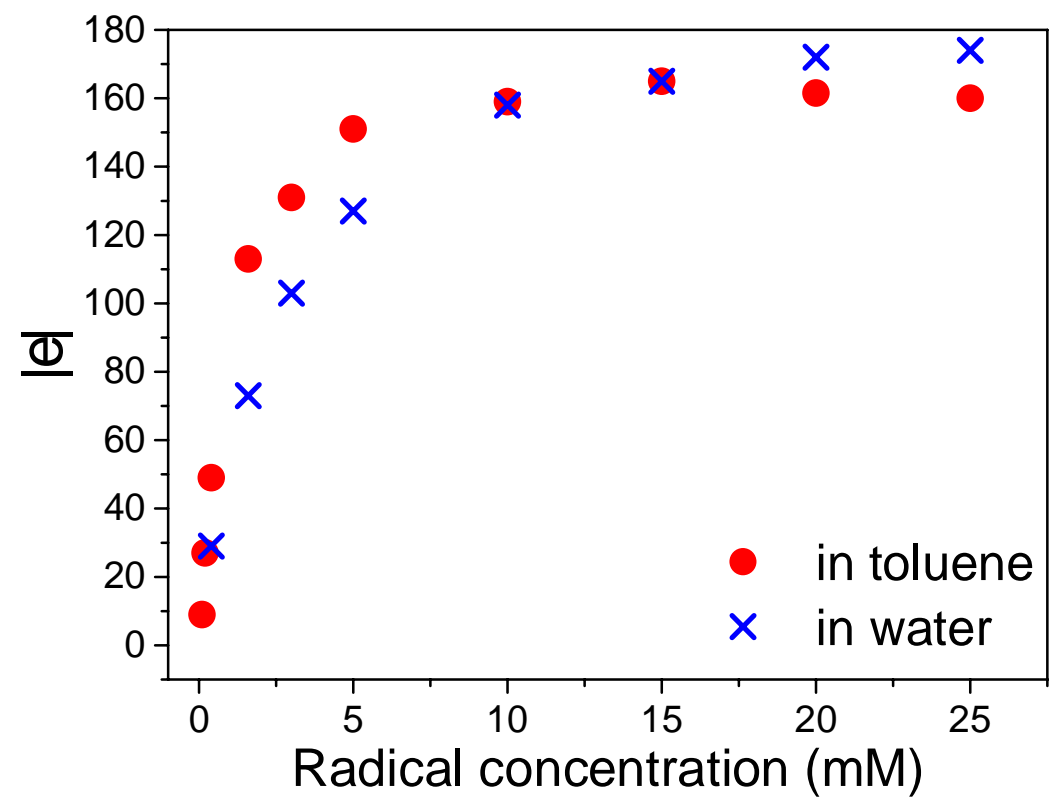

Figure 4.2: (a) ${ }^{1} \mathrm{H}$ NMR spectra of toluene doped with $5 \mathrm{mM}$ TEMPONE- $\mathrm{D}_{16^{-}}{ }^{15} \mathrm{~N}$ at $14 \mathrm{MHz}$; (b) concentration dependence of DNP enhancements (from maximum of $\varepsilon_{F I D}$ ) of TEMPONE-D 16- $^{-}$ ${ }^{15} \mathrm{~N}$ in toluene and in water.

Heating effects due to MW irradiation must be always taken into account in the DNP experiment. The rise in sample temperature leads to larger coupling factor $\xi$ of the system and consequently larger DNP enhancement $\varepsilon^{81}$. In aqueous solution at $9 \mathrm{GHz}$ the heating effect is a result of MW electric field influence $93,136,217,218$. Heating effects are usually manifested through a dependence of DNP signal on duration time of MW excitation. In the case of no heating, the build-up time $T_{\text {buildup }}$ of DNP effect is the spin relaxation time 


\section{Comparative DNP studies in water and toluene: effect of solvent}

of the detected nucleus $T_{1 n}$ in presence of polarizing agent ${ }^{219}$. In result of the optimisation procedure ${ }^{93}$ the measured build-up times are similar to $T_{1 n}$ (within an error of $<10 \%$ ) for all samples in the tubes with internal diameter of $3 \mathrm{~mm}$. The $T_{1 n}$ were measured independently by the NMR inversion recovery experiment.

4.2.2. Leakage factor. The measured spin relaxation rates of the ring and methyl protons of toluene in the case of an absence of the radical are $0.06 \mathrm{~s}^{-1}$ and $0.1 \mathrm{~s}^{-1}$, respectively ${ }^{100}$, whereas the latter one of a water proton is $0.33 \mathrm{~s}^{-1} 81$. Based on the measured relaxation times of the toluene protons in solution at different radical concentrations, the ring protons exhibit slightly higher leakage factors $f$ in comparison with the methyl protons for all cases ${ }^{100}$. The faster proton spin relaxation of water is a reason for the smaller leakage compared to the corresponded values for toluene (Fig. 4.3). The average relaxation rate of the toluene proton in the absence of the radical and relaxivity in toluene-TEMPO system, which are obtained from the time dependence of the first point of the FID signal in NMR inversion recovery experiment, are $0.077 \mathrm{~s}^{-1}$ and $0.133 \mathrm{~s}^{-1} \cdot \mathrm{mM}^{-1}$, respectively. The relaxivity for the water-TEMPO system amounts to $0.284 \mathrm{~s}^{-1} \cdot \mathrm{mM}^{-181}(2.41)$.

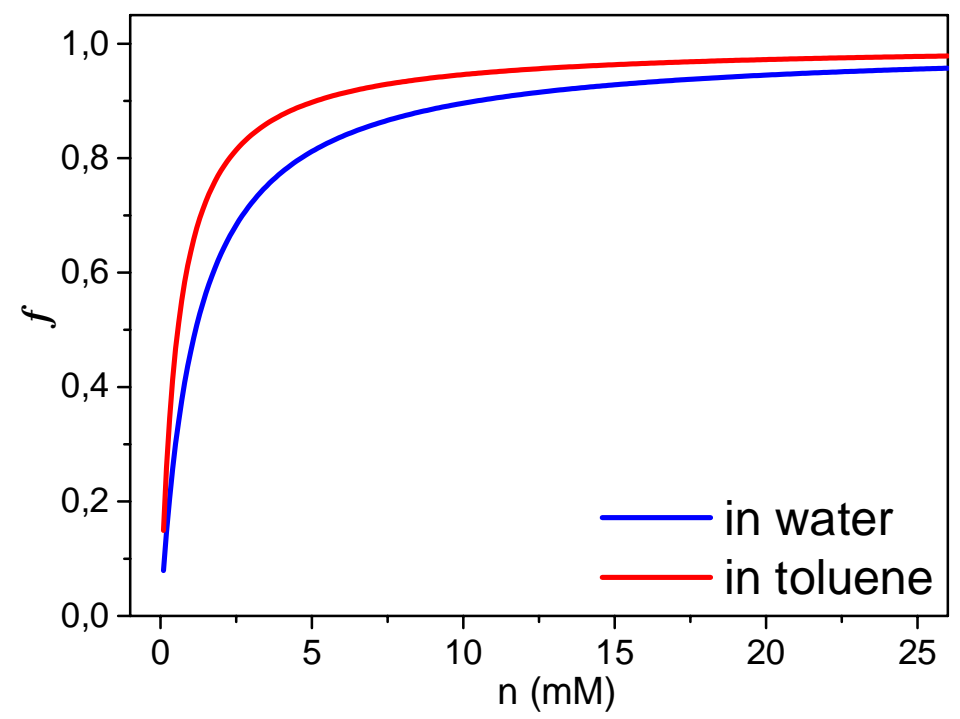

Figure 4.3: Concentration dependence of the leakage factor $f$ in toluene and water doped with TEMPONE- $\mathrm{D}_{16^{-}}{ }^{15} \mathrm{~N}$ at $0.35 \mathrm{~T}$. 


\section{Comparative DNP studies in water and toluene: effect of solvent}

4.2.3. Saturation factor: the role of Heisenberg spin exchange. The concentration dependence of the DNP enhancements of toluene and water protons in the presence of TEMPONE- $\mathrm{D}_{16-}{ }^{15} \mathrm{~N}$ suggests similar coupling factor for both solvents but different behaviours of the saturation factors. To clarify this, the ELDOR method was applied to measure directly the saturation factors of all EPR hyperfine lines and the effective saturation factor as an arithmetic mean of the saturation factors of the individual lines $\left(s_{\text {eff }}=\left(s_{1}+s_{2}\right) / 2\right)$.

Preconditions for this dual frequency experiment are the detection of the radical FID as well as the capability to pump and detect both hyperfine lines within the EPR resonator. For a separation of $\sim 60 \mathrm{MHz}$ of the ${ }^{15} \mathrm{~N}$ nitroxide hyperfine lines, the experiment is well feasible at $9 \mathrm{GHz}$ within the band width of a standard overcoupled dielectric resonator. On the other hand, the detection of an EPR-FID depends on the electron spin-spin relaxation time $T_{2 e}$, which is usually shorter at higher concentrations due to concentration dependent relaxation contributions (exchange and dipolar couplings). According to the $T_{2 e}$ values extracted from the peak-to-peak line widths of the EPR spectra (Fig. 4.1a), FID detection of TEMPONE-D ${ }_{16}{ }^{15} \mathrm{~N}$ in toluene is only possible at concentrations up to about $10 \mathrm{mM}$.

As it is described in Chapter 3, an ELDOR spectrum indicates saturation changes of a fixed EPR transition resulting from irradiation on another EPR transition. Since TEMPONE- $\mathrm{D}_{16}{ }^{15} \mathrm{~N}$ has just two hyperfine EPR transitions (due to hyperfine interaction with ${ }^{15} \mathrm{~N}$ nucleus of spin number $1 / 2$ ) and they can be considered as equivalent under the given experimental conditions (room temperature and low magnetic field $0.35 \mathrm{~T}$ ), the corresponded two drops in the ELDOR spectrum are given by the saturations of two different EPR lines at a fixed irradiated transition (Fig. 4.4a). It simplifies the experimental procedure. The ELDOR measurements were recorded using the MW pumping pulse with magnetic field strength $B_{1} \sim 2-3 \mathrm{G}$ during $1 \mu \mathrm{s}$ and $16 \mathrm{~ns}$ length detecting pulse.

According to the theoretical model $(2.19,2.23,2.25)$, the ratio of the FID drops is constant for the observed system and does not depend on the saturation of the irradiated 


\section{Comparative DNP studies in water and toluene: effect of solvent}

line. Availability of the maximum saturation value $\left(s_{2, \max }\right)$ of the observed EPR line require a complete saturation of the pumped line $\left(s_{l}=1\right)$. On the one hand, the complete FID drop of the irradiated line, which is achieved in all investigated samples, satisfies well the condition $s_{1}=1$ in the ELDOR experiments and, consequently, the second drop of the normalized ELDOR spectra affords $s_{2, \max }$. On the other hand, this condition has been tested in the DNP setup by recording a dependence of EPR line intensity on an applied MW power (Fig. 4.1b). For MW power $\approx 3 \mathrm{~W}$, which is applied for the excitation in DNP experiments, the saturation factor $s_{1}$ near to unity has been achieved in all investigated samples. Thus, the saturation factors obtained from the ELDOR experiments can be used to calculate the effective saturation factors for DNP. As a result, it was demonstrated that $s_{2}$ for TEMPONE- $\mathrm{D}_{16}{ }^{15} \mathrm{~N}$ in toluene increases rapidly at low radical concentrations and is larger in comparison with the water solution of the same concentration (Fig. 4.4b). 


\section{Comparative DNP studies in water and toluene: effect of solvent}

a)

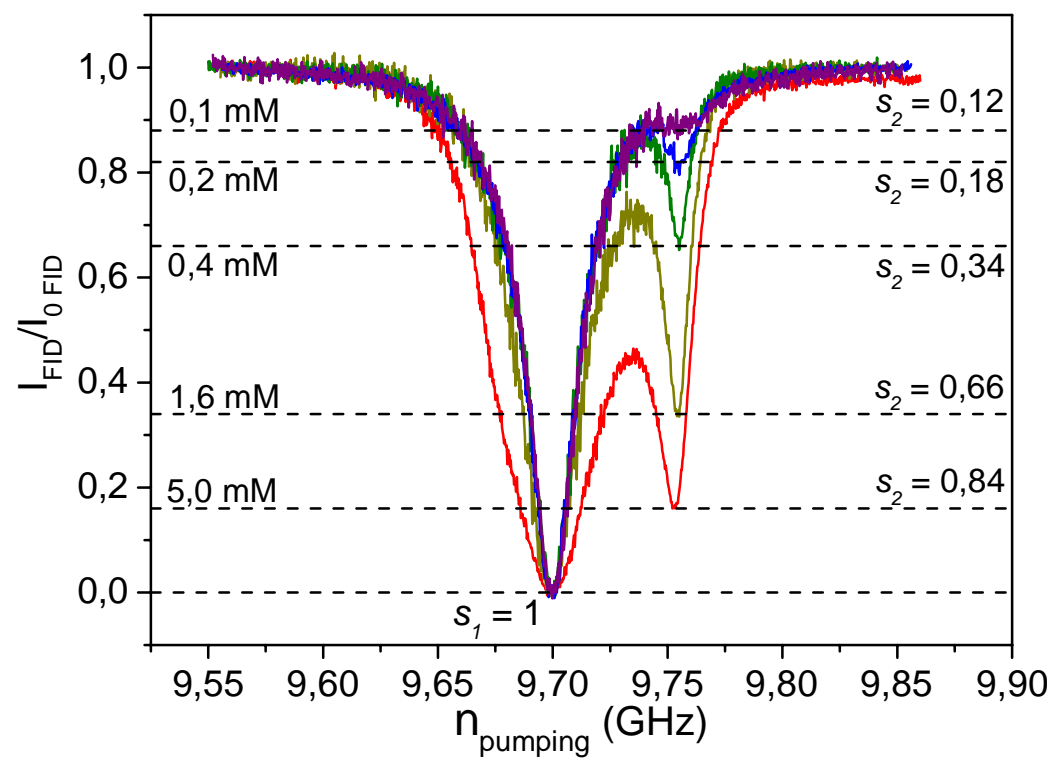

b)

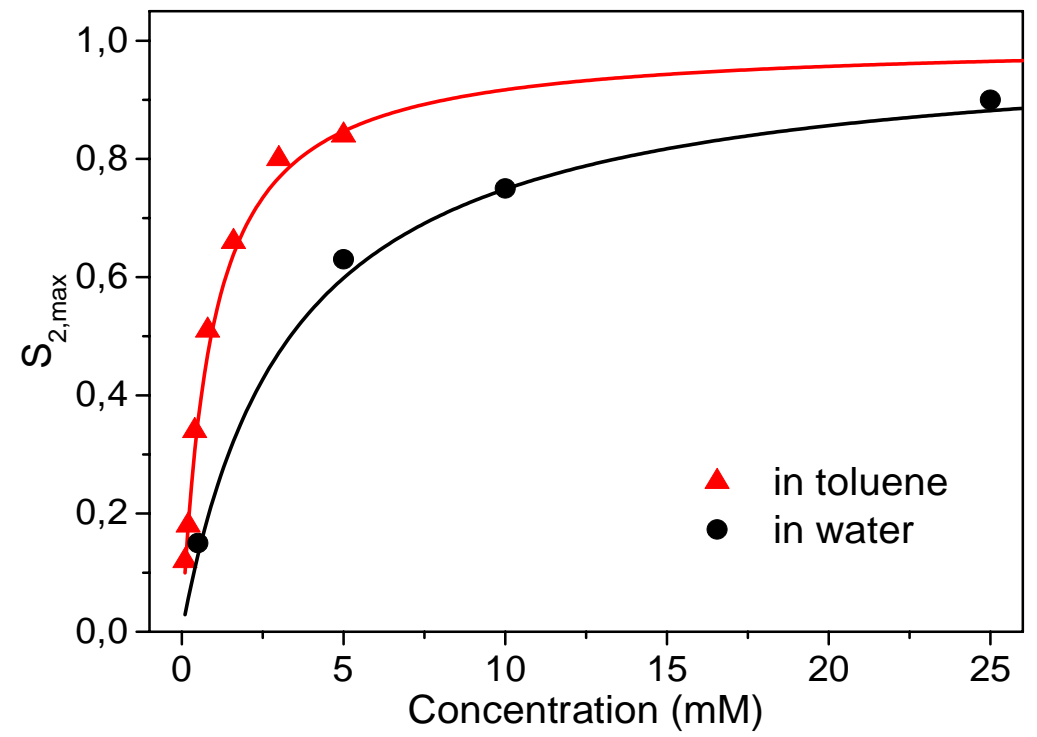

Figure 4.4: (a) EPR-FID intensity of the high-field hyperfine line of TEMPONE- $D_{16^{-}}{ }^{15} \mathrm{~N}$ as a function of the frequency of the saturating (pumping) frequency. (b) Concentration dependence of $s_{2, \max }$, the solid curves are the plots of the equation (4.1) for toluene and water systems with the kinetic parameters obtained from saturation recovery experiments ${ }^{73}$.

The saturation of the EPR line hyperfine coupled with the pumped line results from the nuclear relaxation of ${ }^{15} \mathrm{~N}$ and Heisenberg spin exchange (HE) between the spin states of the two hyperfine lines. The $s_{2, \max }$ is described by following formula (Charpter 2):

$$
s_{2, \max }=1-\frac{1}{1+\frac{1}{4 w_{e}}\left(2 w_{n}+\omega_{e x}\right)},
$$




\section{Comparative DNP studies in water and toluene: effect of solvent}

where $2 w_{e}=1 / T_{1 e}$ and $2 w_{n}=1 / T_{1 n}\left({ }^{15} \mathrm{~N}\right)$ are electron and nuclear $\left({ }^{15} \mathrm{~N}\right)$ transition rates, respectively, and $\omega_{e x}=K_{x} \cdot N$ is the Heisenberg spin exchange rate which depends on the radical concentration $(N)$. An appearance of nonzero $s_{2}>0$ of TEMPO radicals in water solution has been attributed to the $\mathrm{HE}^{73}$. To examine the transitions and $\mathrm{HE}$ rates the saturation recovery of the hyperfine lines has been analysed using ELDOR experiments with variable time interval between the pumping and detecting pulses. The time evolutions of the EPR hyperfine lines after a saturating pulse are described by (Charpter 2)

$$
\left(\begin{array}{l}
i_{1} \\
i_{2}
\end{array}\right)=A\left(\begin{array}{l}
1 \\
1
\end{array}\right) e^{-2 w_{e} t}+B\left(\begin{array}{c}
1 \\
-1
\end{array}\right) e^{-\left(2 w_{e}+2 w_{n}+K_{x} N\right) t}+\left(\begin{array}{l}
i_{1 B}^{*} \\
i_{2 B}^{*}
\end{array}\right),
$$

where $i_{1 B}^{*}$ and $i_{2 B}^{*}$ are steady state signal intensities; the amplitudes $A$ and $B$ are given by the initial FID intensities. The polarization recovery data have been recorded at the same experimental conditions and using the same setup as for the ELDOR measurements (Fig. 4.5). The time evolution fitting allows estimating $2 w_{e}$ and $2 w_{n}+\omega_{e x}$ separately. After assuming $2 w_{n} \ll \omega_{e x}$ as well as in water solution case ${ }^{73}$, the obtained parameters are $T_{1 e}=442 \pm 50 \mathrm{~ns}$ and $\omega_{e x}=(8 \pm 1) \times 10^{6} \mathrm{~s}^{-1}$ (consequently, normalized per mole HE rate $\left.K_{x}=(5.0 \pm 0.7) \times 10^{9} \mathrm{~s}^{-1} \mathrm{M}^{-1}\right)$. It is important to remember that the linear dependence of HE rate on the radical concentration is not always valid ${ }^{220,221 .}$ 


\section{Comparative DNP studies in water and toluene: effect of solvent}

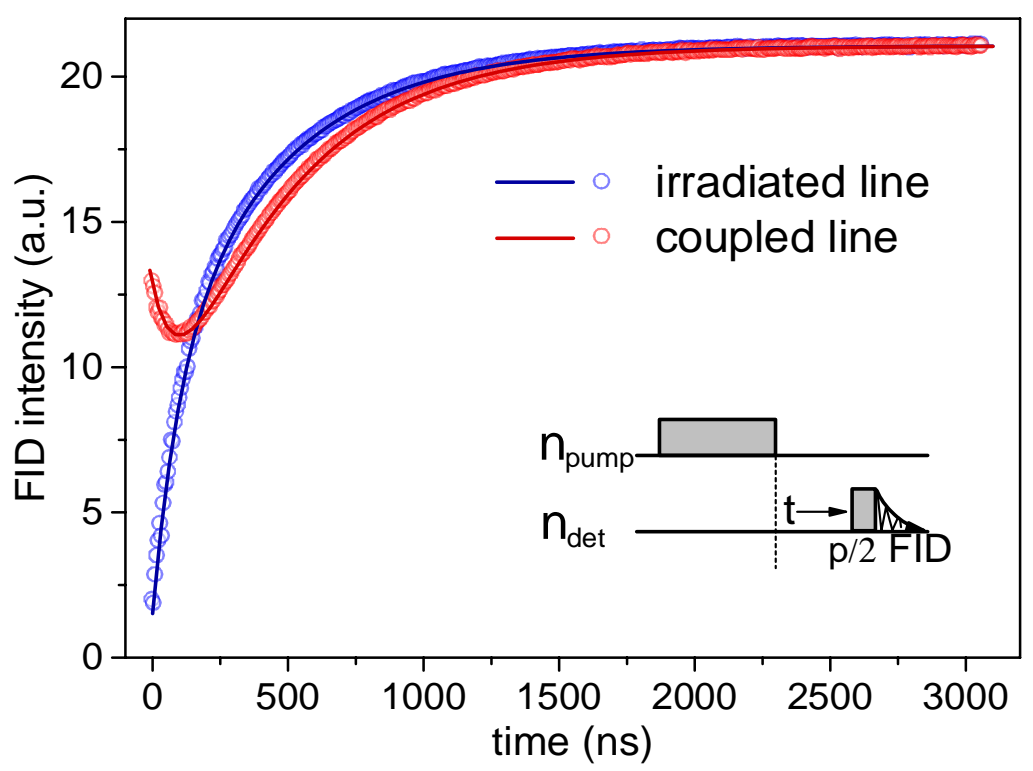

Figure 4.5: Polarization recovery curves and fits of the irradiated EPR line (blue) and the hyperfine coupled line (red) at $1.6 \mathrm{mM}$ TEMPONE- $\mathrm{D}_{16^{-}}{ }^{15} \mathrm{~N}$ in toluene.

To test whether $\omega_{e x}$ accounts for the concentration dependence of $s_{2, \max }$, the latter behavior was simulated according to the equation (4.2) and the assumption $\omega_{e x}=K_{x} \cdot N$. Using the rates values calculated from the saturation recovery it was shown that $s_{2, \max }$ are reproduced in the investigated radical concentration range (Fig. 4.4b). As it is noted, the normalized HE rate per mole in toluene is larger than that in water solution by factor 2.5 73. Heisenberg exchange has been previously treated as a bimolecular interaction with a rate proportional to the translational diffusion coefficient: $\omega_{e x} \sim D^{165,222}$. For Brownian motion in liquids, the latter is in turn inversely proportional to the viscosity $\eta$ through the Stokes-Einstein relation: $D \sim 1 / \eta$. In the strong exchange limit the coupling constant reduces to $K_{x}=\omega_{e x} / N=8 k T / 3 \eta^{165,222}$. An estimation for toluene within this limit leads to $K_{x}=1.1 \times 10^{10} \mathrm{~s}^{-1} \mathrm{M}^{-1}$. The value exceeds the experimental one by a factor of 2 , which in turn seems to be consistent with the trend reported in ref. ${ }^{165}$ when comparing theory with experiment. As compared to water, at room temperature $\left(20{ }^{\circ} \mathrm{C}\right)$ the viscosities are $1.0 \mathrm{mPa} \cdot \mathrm{s}$ and $0.590 \mathrm{mPa} \cdot \mathrm{s}$ for water and toluene, respectively. Clearly, the difference in viscosities accounts only partially for the difference in exchange coupling rates and accordingly the saturation factors. The exchange in water, which is weaker than in toluene, is likely not well reproduced by the classical model of strong exchange coupling. This is somehow not surprising given the different electronic structures of the two 


\section{Comparative DNP studies in water and toluene: effect of solvent}

solvents, specifically the delocalized electronic system in toluene. Recent quantum chemical calculations pointed out the role of electron delocalization effects in throughbond and through-space electron-electron interactions ${ }^{223}$. Values of the exchange constants similar to that of the TEMPONE-toluene system were reported for TEMPO in benzene and di-fluorobenzene ${ }^{216}$.

4.2.4. Coupling factor. With the availability of all factors (Table 4.1) in Overhauser equation (2.9) except $\boldsymbol{\xi}$, we were then able to determine the coupling factors for the ring and methyl ${ }^{1} \mathrm{H}$ of toluene doped with TEMPONE at $0.35 \mathrm{~T}$. This value was calculated at each concentration (Table 4.1). As expected, $\boldsymbol{\xi}$ is independent of the radical concentration (however, at low concentration it can be underestimated due to insufficient irradiation time) within the error limit and averages to 0.27 and 0.24 for the ring and the methyl ${ }^{1} \mathrm{Hs}$, respectively. The error is estimated to be \pm 0.025 (about 10\%) and is larger at lower concentrations due to the weaker $\mathrm{S} / \mathrm{N}$ ratio. However, the error in trend (difference between the average $\xi$ of the two ${ }^{1} \mathrm{H}$ types) is much less, as seen from the data. Therefore the difference between the $\xi_{\mathrm{s}}$ is significant.

The magnitude of $\boldsymbol{\xi}$ for the ${ }^{1} \mathrm{Hs}$ of toluene is less than that of the water protons $\left(\boldsymbol{\xi}_{\text {water }}=0.33\right)$ using the same radical polarizer ${ }^{73}$. One might not exclude a priori that the observed $\xi$ results from counteracting scalar and dipolar relaxation mechanisms, although the dipolar mechanism is reportedly dominant for ${ }^{1} \mathrm{H}$. If pure dipolar relaxation is dominated by a single correlation function, the coupling factor can be estimated from the nuclear relaxation rates (Chapter 2) ${ }^{7}$ :

$$
\xi \approx \frac{5}{7}\left\{1-\frac{2 w_{I}}{R_{I}-R_{I}^{0}}\right\}
$$

where $R_{I}^{0}$ is the nuclear relaxation rate without a paramagnet, $R_{I}$ and $2 w_{I}$ are the relaxation rates at the observing field and the high field limit, respectively. Inserting for the ring protons $R_{I}^{0}=0.06 \mathrm{~s}^{-1}, R_{I}($ at $5 \mathrm{mM})=0.714 \mathrm{~s}^{-1}$ (Table 4.1) and $2 w_{I}$ (at $5 \mathrm{mM}$, $300 \mathrm{MHz}{ }^{1} \mathrm{H} \mathrm{NMR}$ ) $\approx 0.42 \mathrm{~s}^{-1}$ from ref. ${ }^{100}$ we arrive at $\boldsymbol{\xi} \approx 0.25$ (within $10-15 \%$ error limit), which is very close to the experimental value. Therefore, the reduction of the 


\section{Comparative DNP studies in water and toluene: effect of solvent}

coupling factor of the toluene protons is consistent with a DNP mechanism dominated by dipolar relaxation. A more precise interpretation of relaxation contributions requires NMRD (relaxation dispersion) analysis.

Our results reveal a difference in the coupling factors between the ring and methyl protons that has not been inferred to date experimentally due to the lack of information about the saturation behaviour. In a previous $94 \mathrm{GHz} / 3 \mathrm{~T}$ study on TEMPOL/toluene ${ }^{100}$, larger DNP enhancements of the ring protons were observed but attributed to a favourable leakage factor. The present results are mechanistically significant as the obtained coupling factors are in excellent agreement with recent theoretical predictions 224 ( $\xi_{\text {ring }}^{\text {theory }}=0.268 ; \xi_{\text {methyl }}^{\text {theory }}=0.25$ at $9.6 \mathrm{GHz} \mathrm{MW}$ frequency) performed using a sophisticated combination of MD simulations and analytical expressions of spectral densities for dipolar relaxation in the TEMPOL/toluene system. The MD trajectories allowed to capture the dynamics of the polarizer and the solvent within the short interaction range $(<1 \mathrm{~nm})$ and to extract appropriate dipolar correlation functions. The larger $\xi^{\text {theor }}$ of the ring protons was found to be associated to a larger radial density distribution at the closest possible distance to the electron spin of the nitroxide group. The agreement between our experiment and theory at this level of atomistic details is encouraging and opens up new prospects for the application of DNP to investigate molecular dynamics.

Table 4.1: Summary of DNP parameters for toluene doped with TEMPONE- $\mathrm{D}_{16^{-}}{ }^{15} \mathrm{~N}$ ( ${ }^{\mathrm{a}}$ calculated from $T_{\text {buildup }}$ and ${ }^{\mathrm{b}}$ from EPR line width, Fig. 4.1a).

\begin{tabular}{|c|c|c|c|c|c|c|c|c|c|c|c|c|c|}
\hline \multirow{2}{*}{$\begin{array}{c}N \\
(\mathbf{m M})\end{array}$} & \multicolumn{2}{|c|}{$\varepsilon$} & \multicolumn{2}{|c|}{$\begin{array}{c}T_{\text {buildup }} \\
\text { (s) }\end{array}$} & \multicolumn{2}{|c|}{$T_{1 \mathrm{n}}(\mathrm{s})$} & \multicolumn{2}{|c|}{$f$} & \multirow{2}{*}{$\begin{array}{l}{ }^{b} T_{2 \mathrm{e}} \\
(\mathrm{ns})\end{array}$} & \multirow[t]{2}{*}{$S_{2 \max }$} & \multirow[t]{2}{*}{$s_{\text {eff }}$} & \multicolumn{2}{|c|}{$\xi$} \\
\hline & $\mathrm{H}_{\text {ring }}$ & $\mathrm{H}_{\mathrm{m}}$ & $\mathrm{H}_{\text {ring }}$ & $\mathrm{H}_{\mathrm{m}}$ & $\mathrm{H}_{\text {ring }}$ & $\mathrm{H}_{\mathrm{m}}$ & $\mathrm{H}_{\text {ring }}$ & $\mathrm{H}_{\mathrm{m}}$ & & & & $\mathrm{H}_{\text {ring }}$ & $\mathrm{H}_{\mathrm{m}}$ \\
\hline 0.2 & 32 & 18 & 12.1 & 8.9 & 10.7 & 7.4 & 0.34 & 0.24 & 171 & 0.18 & 0.59 & 0.25 & 0.21 \\
\hline 0.4 & 58 & 34 & 8.0 & 6.4 & - & - & $0.51^{\mathrm{a}}$ & $0.34^{\mathrm{a}}$ & 152 & 0.34 & 0.67 & 0.26 & 0.23 \\
\hline 1.6 & 124 & 94 & 3.3 & 3.1 & 3.2 & 3.2 & 0.80 & 0.67 & 98 & 0.66 & 0.83 & 0.28 & 0.26 \\
\hline 5.0 & 159 & 137 & 1.2 & 1.3 & 1.4 & 1.6 & 0.91 & 0.84 & 48 & 0.84 & 0.92 & 0.29 & 0.26 \\
\hline
\end{tabular}




\section{Comparative DNP studies in water and toluene: effect of solvent}

\subsection{Conclusion}

The DNP experiments with TEMPONE in toluene at $0.35 \mathrm{~T}$ demonstrate the possibility to achieve large signal enhancements $(\varepsilon \geq 100)$ at low polarizer concentrations $(\leq 5 \mathrm{mM})$, which makes this solvent appropriate for developments and applications of DNP. The high DNP efficiency is related to the highly effective saturation factor. The latter one occurs due to a Heisenberg spin exchange process between two existent EPR hyperfine lines, whose rate is larger than that in water by a factor of $\geq 2$. The coupling factors calculated from the experimental results of the other characteristic DNP parameters support a recent theoretical MD investigation, by which the coupling factors in toluene reflect the detailed motion of the solvent protons around the atoms bearing the electron spin. 


\section{DNP with fullerene-nitroxide derivatives: effect of polarizer's size}

\section{DNP with fullerene-nitroxide derivatives: effect of polarizer's size}

It was not clear whether an attachment of $\mathrm{C}_{60}$, as the dye, to the commonly employed polarizers, such as TEMPO radicals, can reduce or destroy their efficiency for DNP applications.

\subsection{Results and analysis}

5.1.1. Determination of rotational correlation time. The EPR spectra of the investigated molecules (TEMPOL and fullerene-nitroxide derivatives) in degassed toluene solutions show three well-resolved hyperfine lines (due to the interaction with ${ }^{14} \mathrm{~N}$ which has nuclear spin $\mathrm{I}=1$ ) (Fig. 5.1a). These spectra are well consistent with the case of the fast motion regime. The software "Easyspin" was used for simulating the EPR spectra in the fast motion regime and determination of the molecular rotational correlation time $\tau_{c}$ for each sample ${ }^{186}$. In spite of that the line widths of TL (TEMPOL) is slightly larger than the ones of $\mathbf{F N}$, which can be a result of some residual oxygen content, the rotational correlation time of $\mathbf{F N}$ still results slower (0.01 ns vs. $0.06 \mathrm{~ns}$ for TL and FN, respectively), as the simulation reflects from the relative intensity of the high field vs central hyperfine lines. 
a)

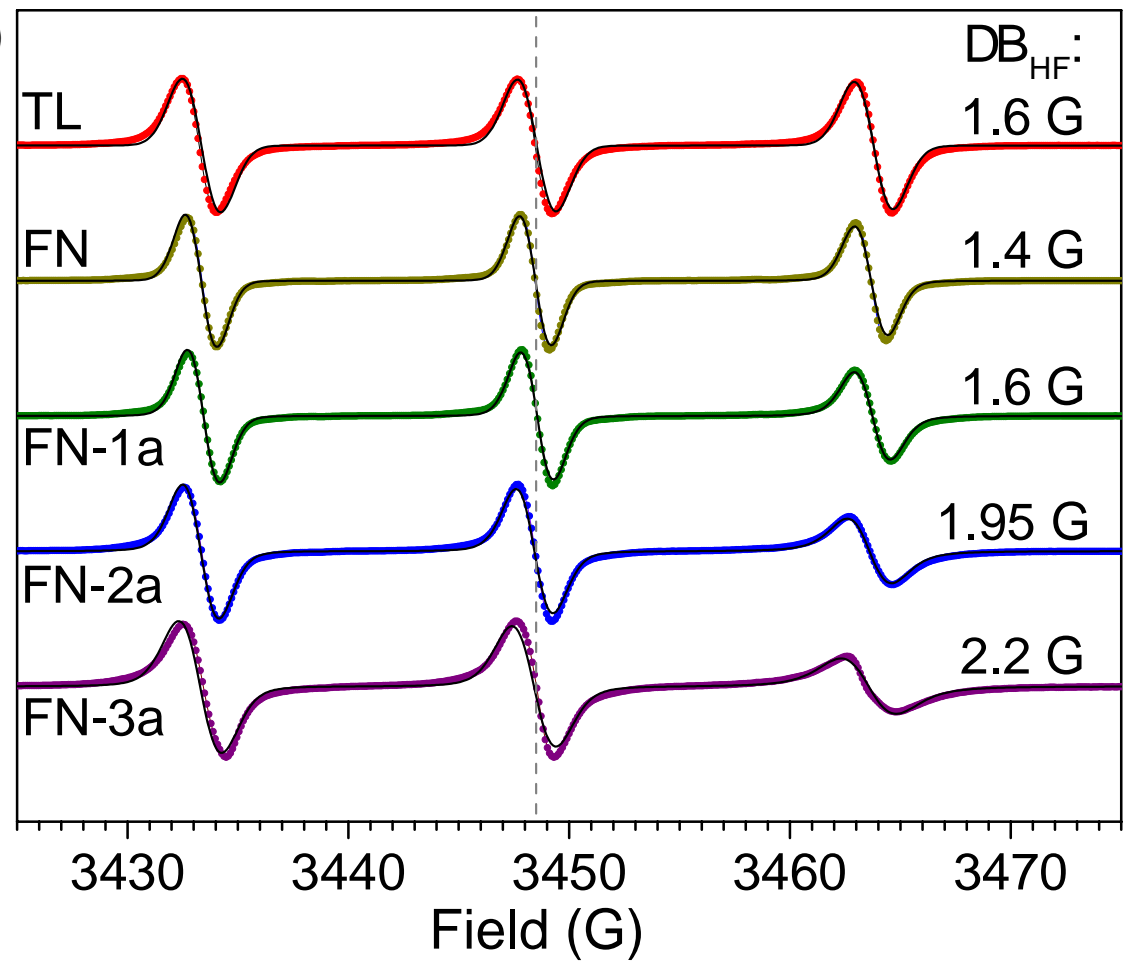

b)

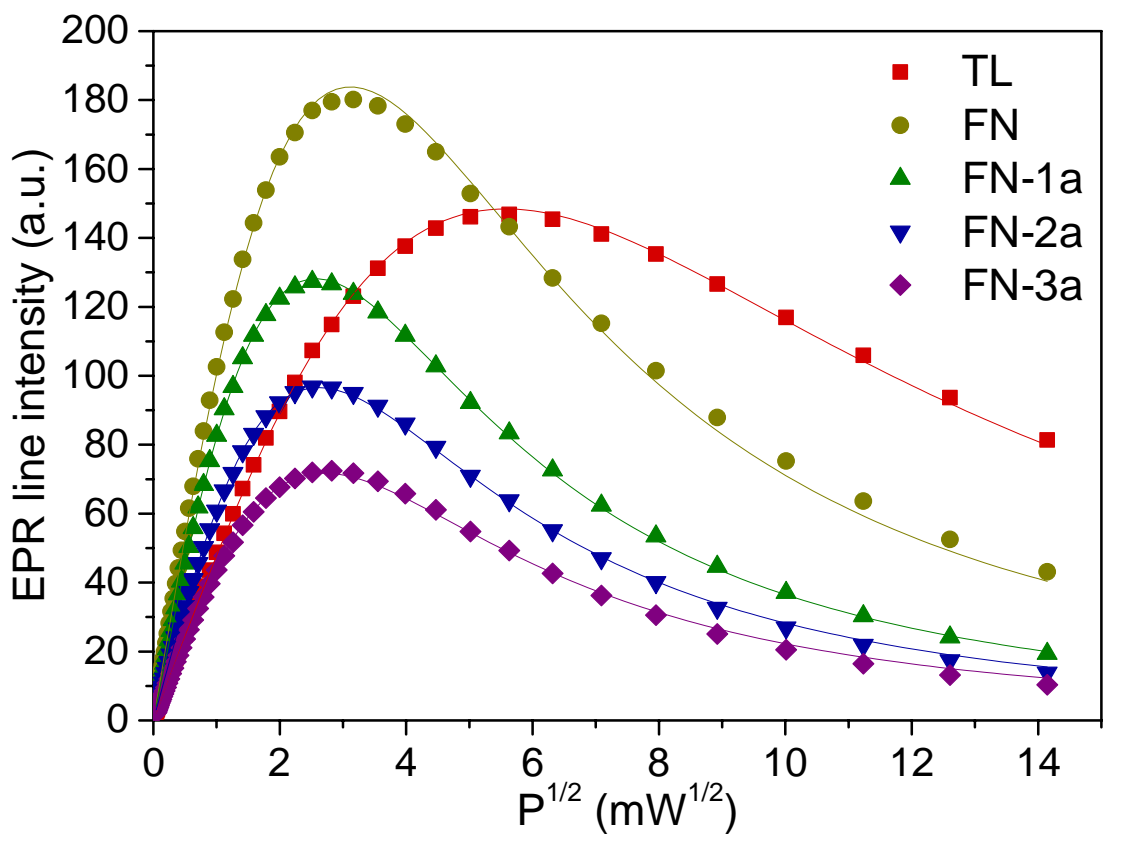

Figure 5.1: (a) Normalized CW-EPR spectra of fullerene-nitroxide derivatives in toluene $(1.5 \mathrm{mM})$. Black solid lines are fits according to the fast motion regime (Chapter 2). At $2 \mathrm{~mW}$ of the MW power, $1 \mathrm{G}$ and $100 \mathrm{kHz}$ of modulation amplitude and frequency, respectively, room temperature. (b) MW power dependence of the peak-to-peak intensity of the central nitroxide hyperfine line. Solid curves are fitting $(2.12)^{70}$. 


\section{DNP with fullerene-nitroxide derivatives: effect of polarizer's size}

5.1.2. DNP experiments. The DNP experiments were performed at $0.35 \mathrm{~T}$ in toluene solutions with $\sim 1.5 \mathrm{mM}$ of all considered polarizers. The used concentration is the maximal achievable for the fullerene-nitroxide derivatives. As in the previous experimental part (Chapter 3) ${ }^{1} \mathrm{H}-\mathrm{NMR}$ spectra have two well-resolved peaks correspondent to the ring and methyl protons. The highest DNP enhancements have been reached at MW irradiation on the central EPR transition of ${ }^{14} \mathrm{~N}$-nitroxide. FN-2a demonstrates the largest DNP enhancements of -110 and -87 for the ring and methyl protons, respectively (Fig. 5.2a). The fullerene-nitroxide derivatives as well as TEMPO have negative enhancements, which indicate the domination of the dipolar relaxation in the mechanism of the spin polarization transfer. The series of DNP experiments demonstrate an enhancement increasing from TL to FN-2a and then a slight decrease in FN-3a (Fig. 5.2b). This behaviour suggests a favourable role of the polarizer's size in DNP process. On the other hand, according to the model, which presumes and describes DNP as mechanism controlled by translational diffusion, the coupling factor should decrease with molecular size ${ }^{81}$. 
a)

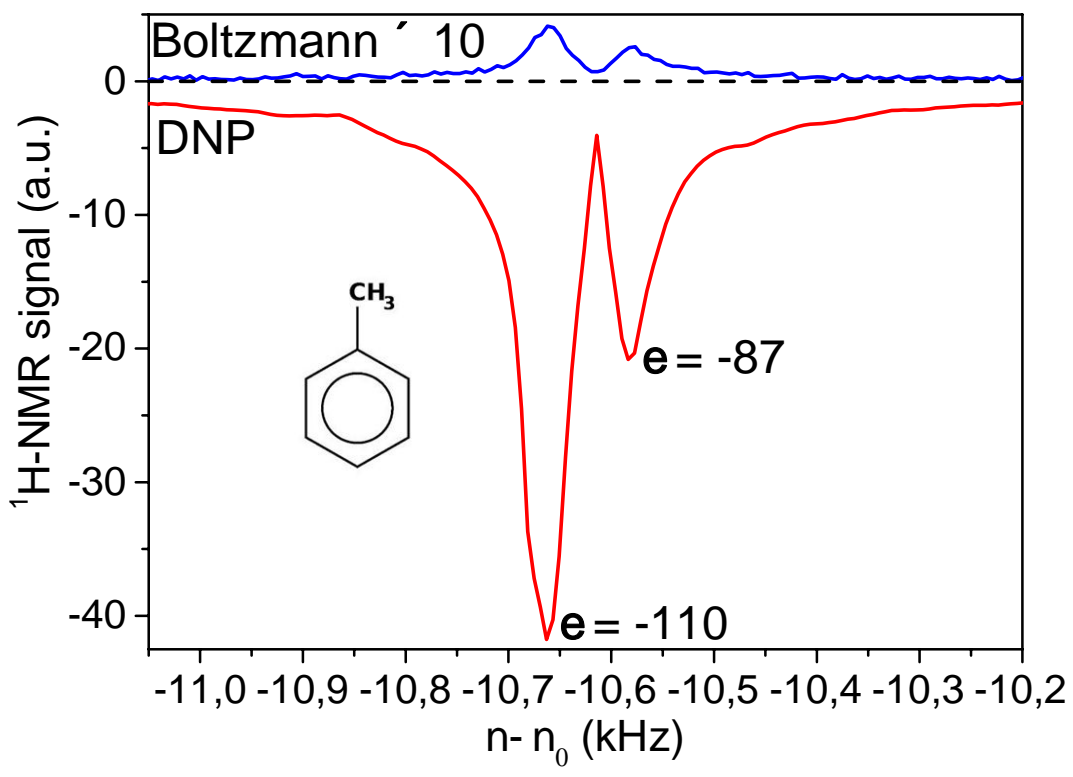

b)

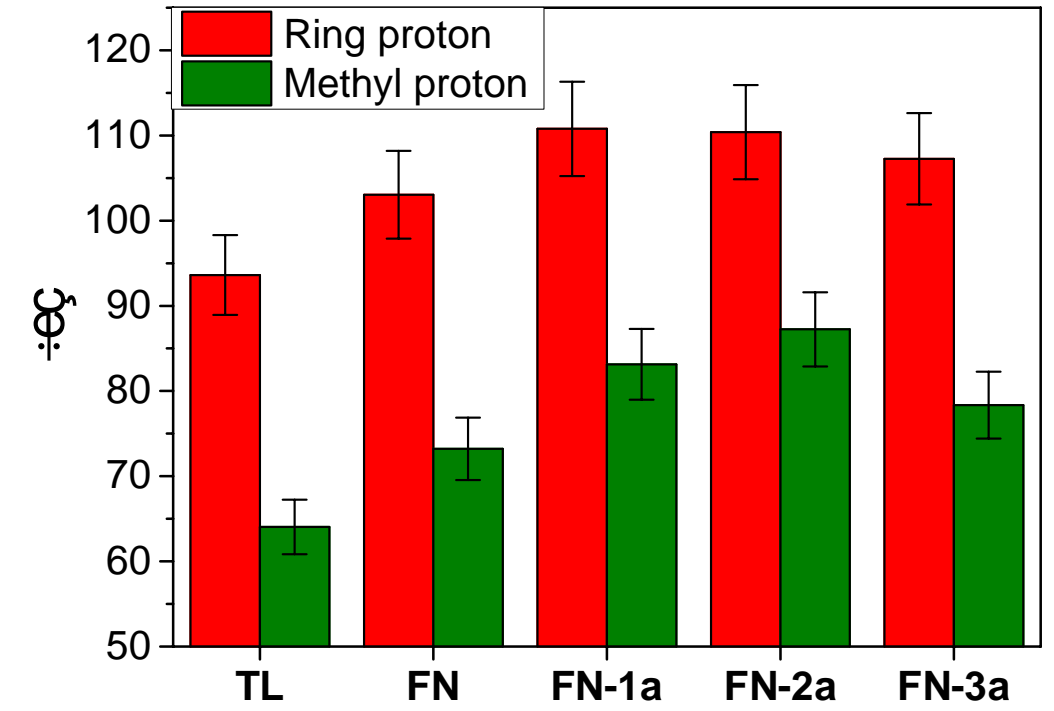

Figure 5.2: (a) Boltzmann (blue) and DNP (red) $14 \mathrm{MHz}{ }^{1} \mathrm{H}-\mathrm{NMR}$ spectra recorded in toluene solution of FN-2a. (b) DNP enhancements for both ring and methyl protons of toluene with different polarizers.

Therefore, understanding the increasing DNP enhancement requires examining all Overhauser parameters and their dependence on polarizer's size. The leakage factors were calculated through the measurement of the protons spin relaxation in presence and absence of the investigated polarizers by inversion recovery experiments. The leakage factor slightly increases from TL to FN-3a (Table 5.2). 


\section{DNP with fullerene-nitroxide derivatives: effect of polarizer's size}

5.1.3. Saturation factor: ELDOR measurements. Pulsed ELDOR was applied to measure the saturation of the three ${ }^{14} \mathrm{~N}$ hyperfine transitions in the EPR. The experimental conditions were the same as in the previous experiments. The effective saturation factor is the arithmetic mean of the saturation of each EPR transition $s_{\text {eff }}=\left(s_{1}+s_{2}+s_{3}\right) / 3\left(s_{1}, s_{2}, s_{3}\right.$ are for the low field, central field and high field hyperfine EPR lines, respectively). The experimental determination of the saturation factors requires three normalized ELDOR spectra with different fixed pumping frequencies. In the case of pumping the central EPR line the central peaks of the red, green and blue curves correspond to $s_{1}, s_{2}$ and $s_{3}$, respectively (Fig. 5.3). Analogous is the case of pumping the other two EPR lines. While for TL (Fig. 5.3a) the ELDOR effect appears not dependent on the choice of the pumped hyperfine line, for the FN-3a (Fig. 5.3e) sample it does and the largest $s_{\text {eff }}$ is observed for pumping on the central hyperfine line, indicating that this is the optimal setup for DNP.

The obtained ELDOR spectra (Fig. 5.3) demonstrate a complete suppression of the FID when the pumping and detecting frequencies coincide for all samples independently on hyperfine EPR line. Therefore, the experimentally obtained saturation factor values correspond to the maximally achievable $s_{\text {eff }}$ in the DNP experiments with pumping just one EPR transition ${ }^{73}, 225,226$. The CW EPR experiments at various applied MW power have demonstrated the complete saturations $(s \approx 1)$ of the irradiated EPR transitions for all investigated samples at $P_{M W} \approx 3 \mathrm{~W}$ (which is applied in the DNP experiments) (Fig. 5.1b). Thus, the saturation factors extracted from the ELDOR experiments are the same as in the DNP experiments. The obtained $s_{\text {eff }}$ values are the largest for all investigated samples when the central hyperfine line is saturated. It has been found that $s_{\text {eff }}$ increases with polarizer's size, with a value of 0.71 for TL up to 0.95 for FN-2a and FN-3a. The saturation factors for the last two samples are unusually very high at low radical concentration $(\sim 1.5 \mathrm{mM})$. For comparison, DNP experiments with TEMPONE$\mathrm{D}_{16^{-}}{ }^{15} \mathrm{~N}$ require a radical concentration of $\geq 5 \mathrm{mM}$ in toluene or $\geq 25 \mathrm{mM}$ in water ${ }^{73,227}$. 


\section{DNP with fullerene-nitroxide derivatives: effect of polarizer's size}
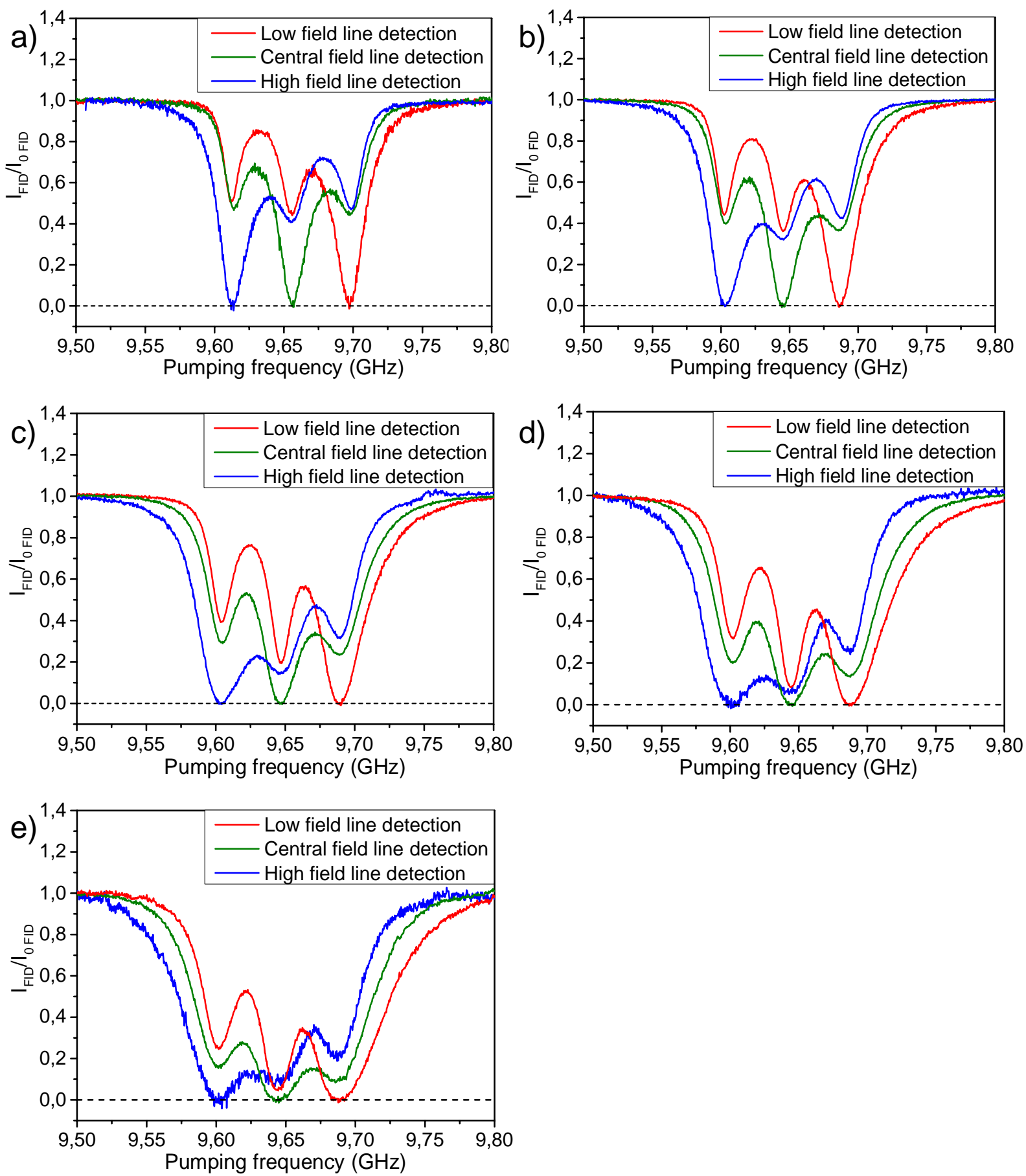

Figure 5.3: Normalized FID intensities of TL (a), FN (b), FN-1a (c), FN-2a (d) and FN-3a (e), respectively, as a function of the pumping frequency.

To investigate the origin of the observed saturation factors the population dynamics of ${ }^{14} \mathrm{~N}$-nitroxides EPR lines have been analysed by the polarization recovery experiments for all investigated samples (Fig. 5.4) using ELDOR techniques 73, 166, 221, 227 . The experimental conditions are the same as for saturation factor measurements except pumping pulse duration. To avoid missing information on the initial dynamics, the 
investigated systems should not reach the steady states and a shorter pump pulse should be used as compared to the ELDOR. The time evolutions of the FID signals corresponding to the hyperfine EPR lines after a pumping pulse were derived in

\section{Charpter 2:}

$$
\left(\begin{array}{l}
i_{1} \\
i_{2} \\
i_{3}
\end{array}\right)=A\left(\begin{array}{l}
1 \\
1 \\
1
\end{array}\right) e^{-2 w_{e} t}+B\left(\begin{array}{c}
1 \\
-2 \\
1
\end{array}\right) e^{-\left(2 w_{e}+3 w_{n}+K_{x} N\right) t}+C\left(\begin{array}{c}
1 \\
0 \\
-1
\end{array}\right) e^{-\left(2 w_{e}+w_{n}+K_{x} N\right) t}+\left(\begin{array}{l}
i_{1 B}^{*} \\
i_{2 B}^{*} \\
i_{3 B}^{*}
\end{array}\right)
$$

where $i_{1 B}^{*}, i_{2 B}^{*}$ and $i_{3 B}^{*}$ are steady-state signal intensities; $2 w_{e}=1 / T_{l e}$ and $2 w_{n}=1 / T_{l n}\left({ }^{14} \mathrm{~N}\right)$ are electron and nuclear spin-lattice relaxation rates, respectively; $K_{x}$ is Heisenberg spin exchange (HE) rate per mole and $N$ is the polarizer concentration. The amplitudes $A, B$ and $C$ are given by the initial FID intensities. According to the theoretical model, when the central EPR line is irradiated the signal behaviors (5.1) is reduced from triexponential to bi-exponential (the amplitude $C=0$ ). Thus, the experiments with MW pumping either on the low field or high field EPR transitions are required to estimate all three rates $\left(2 w_{e}, 2 w_{n}\right.$ and $\left.K_{x} N\right)$. 


\section{DNP with fullerene-nitroxide derivatives: effect of polarizer's size}
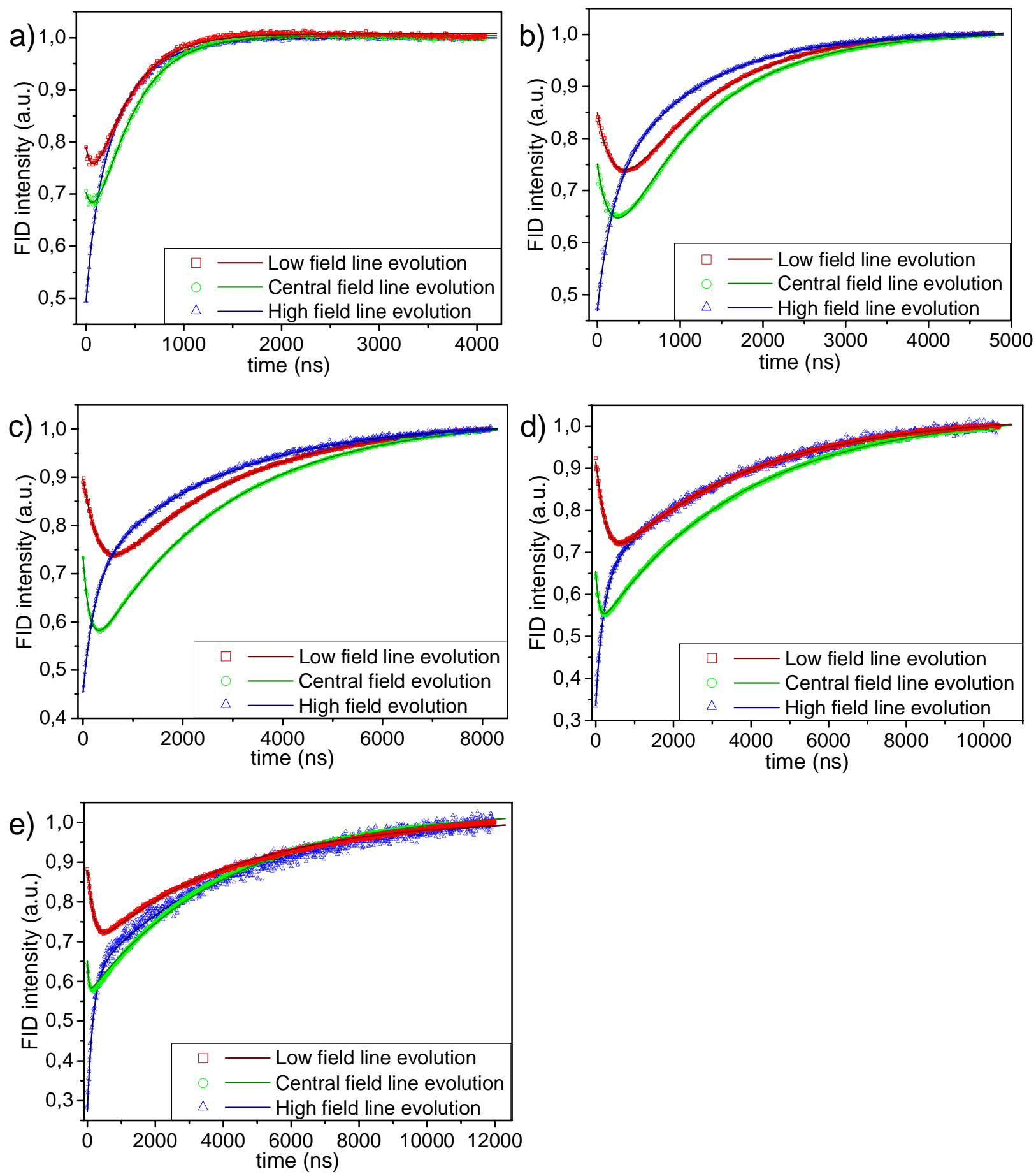

Figure 5.4: Polarization Recovery (PR) curves and fits (solid lines) of the TL (a), FN (b), FN-1a (c), FN-2a (d), FN-3a (e), respectively, for the case of pumping on the high field line $\left(t_{\text {pump }}=0.1 \mu \mathrm{s}\right)$. The polarizer concentration is $\sim 1.5 \mathrm{mM}$.

5.1.4. Discussion: the role of nitrogen nuclear relaxation. The results of the polarization recovery measurements and fitting show that the electron spin-lattice 


\section{DNP with fullerene-nitroxide derivatives: effect of polarizer's size}

relaxation $2 w_{e}$ and Heisenberg spin exchange rate $K_{x} N$ decrease with the polarizer size whereas the nuclear relaxation of the nitrogen ${ }^{14} \mathrm{~N} 2 w_{n}$ increases. To rationalize this phenomena, the characteristic parameters as rotational correlation times $\left(\tau_{c}\right)$ have been calculated from the simulation of CW EPR spectra (Fig. 5.1a) by the model of the fast motion regime (Chapter 2). The ${ }^{14} \mathrm{~N}$ nuclear relaxation increases with the correlation time, while the HE rate decreases (Fig. 5.5a). The same behavior of the nuclear relaxation was found in the studies of TEMPO- ${ }^{15} \mathrm{~N}$ radical in the solvents with different viscosity ${ }^{164}$ and the studies of spin labeled lipids ${ }^{166,221}$. It was proposed that the nitrogen nuclear relaxation is governed by the electron-nuclear dipolar mechanism in the correlation time range from picoseconds to nanoseconds ${ }^{164}$. In this case the nuclear relaxation is contributed from two spectral densities ${ }^{69,164}$ :

$$
\frac{1}{T_{1 n}}=k \cdot\left(\frac{7 \cdot \tau_{c}}{1+\omega_{e}^{2} \tau_{c}^{2}}+\frac{3 \cdot \tau_{c}}{1+\omega_{n}^{2} \tau_{c}^{2}}\right),
$$

where $\omega_{e}$ and $\omega_{n}$ are the electron Larmor and nuclear resonance frequencies, respectively, the latter one is dominated by the hyperfine coupling $\left(\omega_{n} \approx \omega_{A}, \omega_{A} / 2 \pi \approx 20 \mathrm{MHz}\right)$. The rotational correlation times of all investigated samples in toluene solution are $<1 \mathrm{~ns}$, which corresponds to the regime of increasing nuclear relaxation with the correlation time (Fig. 5.5b). 
a)

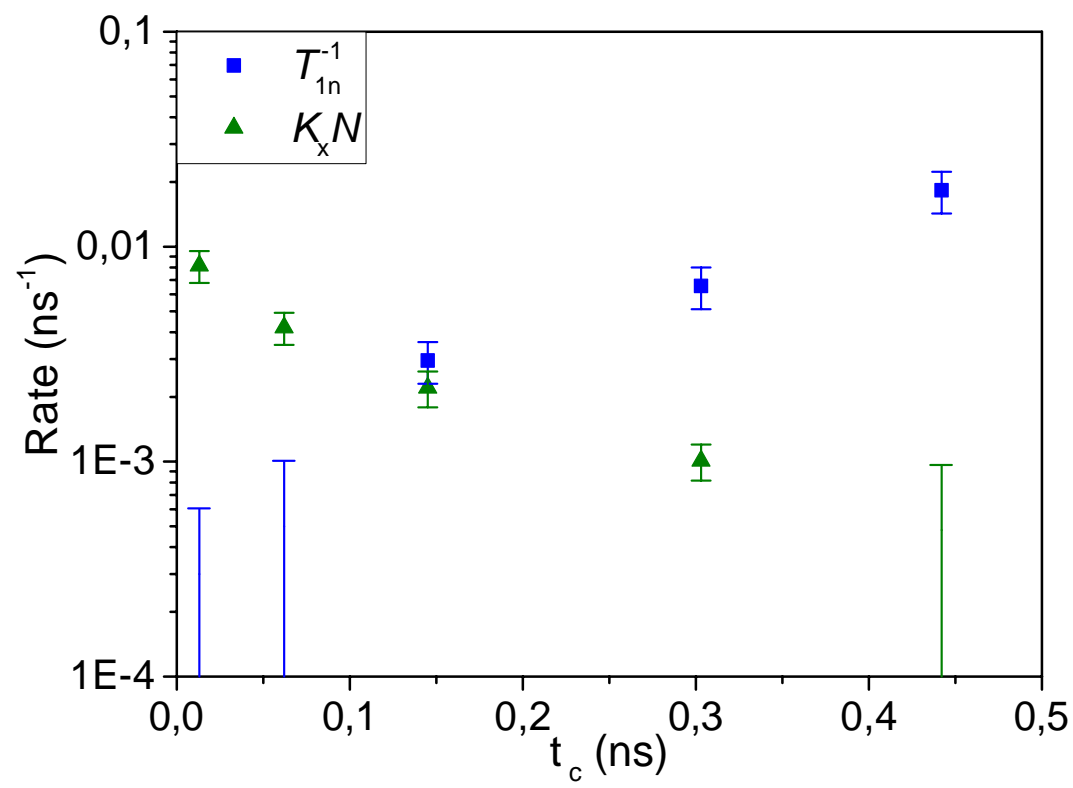

b)

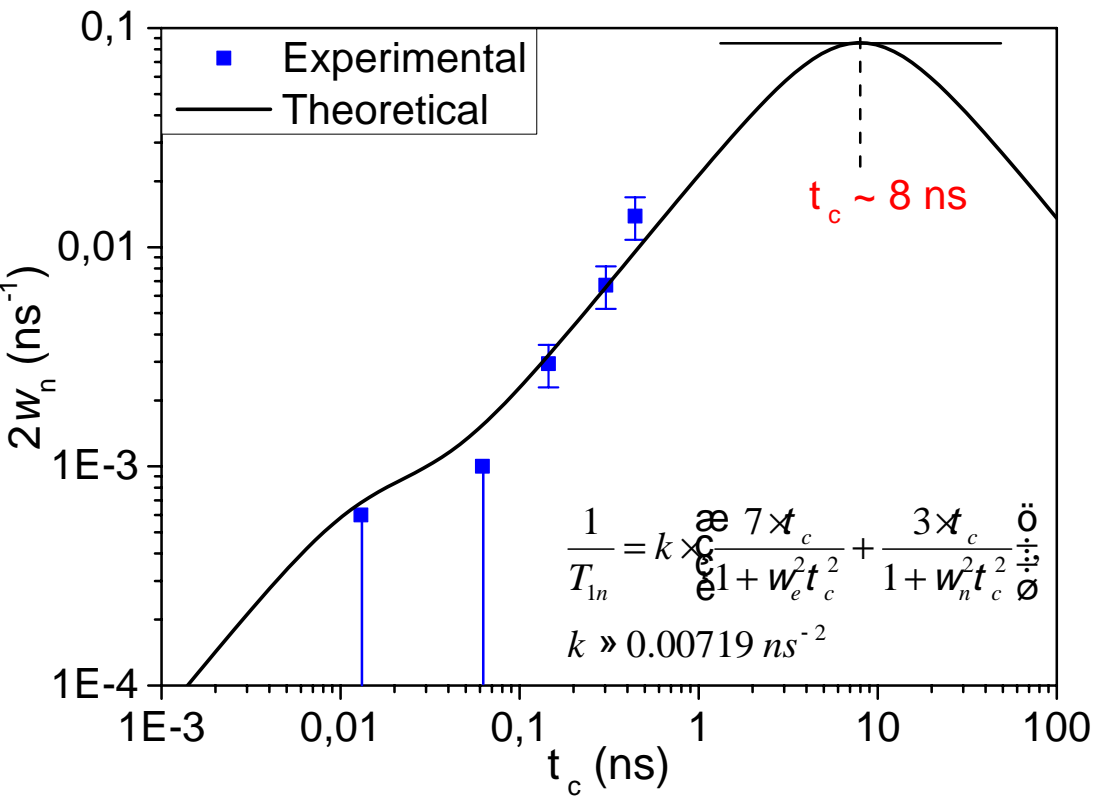

Figure 5.5: (a) ${ }^{14} \mathrm{~N}$ nuclear relaxation $2 w_{n}\left(1 / T_{l n}\right)$ and $\mathrm{HE}$ rate $K_{x} N$ (with polarizer concentration $N \sim 1.5 \mathrm{mM}$ ). $2 w_{n}$ values for TL and $\mathbf{F N}$ and $K_{x} N$ value for FN-3a turned out to be negligible in the fits and are given as upper limit. The errors of up to $20 \%$ were estimated from the experiments with various polarizer concentrations. (b) Simulation of the ${ }^{14} \mathrm{~N}$ nuclear relaxation according to the electron-nuclear dipolar mechanism (5.2).

The theoretical model of spin exchange process in ${ }^{14} \mathrm{~N}$-nitroxide (Chapter 2) and the obtained analytical expressions for saturation factors allow understanding and explaining the influence of ${ }^{14} \mathrm{~N}$ nuclear spin-lattice relaxation on the saturation factor. Independently on the EPR transition chosen for MW irradiation the saturation factors increases with the 
nitrogen spin-lattice relaxation and Heisenberg spin exchange rate but decreases with the electron spin-lattice relaxation (2.23). These three rates are present in the expressions as two ratios

$$
\frac{3 w_{n}+K_{x} N}{2 w_{e}} \text { and } \frac{w_{n}+K_{x} N}{2 w_{e}} .
$$

They show that in spite of the HE rate decreasing with the correlation time/molecular

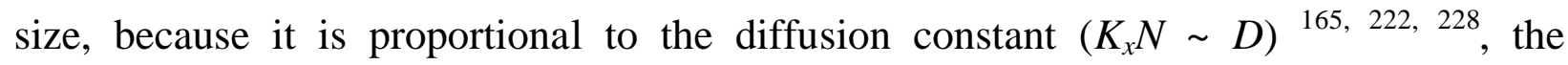
dynamics of the nuclear relaxation $2 w_{n}$ and electron relaxation $2 w_{e}$ explain the increase of the saturation factors $(2.23,2.25)$. The saturation factor values calculated from the analytical expressions, using the rate parameters obtained from the polarization recovery experiments, are in good agreement with the experimentally measured ones by ELDOR. The errors are of 10-15\% (Table 5.1). 


\section{DNP with fullerene-nitroxide derivatives: effect of polarizer's size}

Table 5.1: Comparison of the saturation factor values experimentally determined from ELDOR spectra $\left(s^{e x p}\right)$ and theoretically calculated using the analytical equations and the rate parameters $\left(2 w_{e}, 2 w_{n}, K_{x} N\right)$ obtained from the fitting of polarization recovery curves $\left(\boldsymbol{s}^{\text {theory }}\right)$. LF - low field, $\mathrm{HF}$ - high field.

\begin{tabular}{c|c|c|c|c|c|c}
\hline \multirow{2}{*}{ Sample } & \multicolumn{6}{|c}{ LF line irradiation } \\
\cline { 2 - 7 } & $\boldsymbol{s}_{\mathbf{2}}{ }^{\text {exp }}$ & $\boldsymbol{s}_{\mathbf{2}}{ }^{\text {theory }}$ & $\boldsymbol{s}_{\mathbf{3}}{ }^{\text {exp }}$ & $\boldsymbol{s}_{\mathbf{3}}{ }^{\text {theory }}$ & $\boldsymbol{s}^{\text {exp }}$ & $\boldsymbol{s}^{\text {theory }}$ \\
\hline TL & 0.55 & 0.51 & 0.53 & 0.51 & 0.69 & 0.67 \\
\hline FN & 0.63 & 0.62 & 0.57 & 0.61 & 0.74 & 0.74 \\
\hline FN-1a & 0.76 & 0.81 & 0.68 & 0.76 & 0.82 & 0.85 \\
\hline FN-2a & 0.86 & 0.89 & 0.74 & 0.84 & 0.87 & 0.91 \\
\hline FN-3a & 0.91 & 0.92 & 0.79 & 0.89 & 0.90 & 0.94 \\
\hline
\end{tabular}

\begin{tabular}{c|c|c|c|c|c}
\hline \multirow{2}{*}{ Sample } & \multicolumn{5}{|c}{ Central line irradiation } \\
\cline { 2 - 6 } & $\boldsymbol{s}_{\boldsymbol{1}}^{\text {exp }}$ & $\boldsymbol{s}_{\boldsymbol{1}}^{\text {exp }}$ & $\boldsymbol{s}_{\boldsymbol{1 , 3}}^{\text {theory }}$ & $\boldsymbol{s}^{\text {exp }}$ & $\boldsymbol{s}^{\text {theory }}$ \\
\hline TL & 0.55 & 0.59 & 0.51 & 0.71 & 0.68 \\
\hline FN & 0.64 & 0.68 & 0.62 & 0.77 & 0.75 \\
\hline FN-1a & 0.80 & 0.85 & 0.85 & 0.89 & 0.90 \\
\hline FN-2a & 0.92 & 0.94 & 0.93 & 0.95 & 0.95 \\
\hline FN-3a & 0.95 & 0.91 & 0.96 & 0.95 & 0.97 \\
\hline
\end{tabular}

\begin{tabular}{c|c|c|c|c|c|c}
\hline \multirow{2}{*}{ Sample } & \multicolumn{6}{|c}{ HF line irradiation } \\
\cline { 2 - 7 } & $\boldsymbol{s}_{\boldsymbol{1}}{ }^{\text {exp }}$ & $\boldsymbol{s}_{\boldsymbol{1}}{ }^{\text {theory }}$ & $\boldsymbol{s}_{\boldsymbol{2}}{ }^{\text {exp }}$ & $\boldsymbol{s}_{\boldsymbol{2}}^{\text {theory }}$ & $\boldsymbol{s}^{\text {exp }}$ & $\boldsymbol{s}^{\text {theory }}$ \\
\hline TL & 0.49 & 0.51 & 0.53 & 0.51 & 0.67 & 0.67 \\
\hline FN & 0.56 & 0.61 & 0.60 & 0.62 & 0.72 & 0.74 \\
\hline FN-1a & 0.61 & 0.76 & 0.71 & 0.81 & 0.77 & 0.85 \\
\hline FN-2a & 0.68 & 0.84 & 0.80 & 0.89 & 0.83 & 0.91 \\
\hline FN-3a & 0.75 & 0.89 & 0.84 & 0.92 & 0.86 & 0.94 \\
\hline
\end{tabular}




\section{DNP with fullerene-nitroxide derivatives: effect of polarizer's size}

Heisenberg spin exchange and nuclear relaxation have different dependence on the polarizer concentration. While the HE rate depends, the nitrogen nuclear relaxation does not. The series of experiments with various radical concentrations (Fig. 5.6) demonstrate that the saturation factor of TL increases with polarizer concentration whereas the saturation factor of $\mathbf{F N - 2 a}$ stays nearly constant. These are results of different dominant processes. The HE prevails in the case of TL whereas the nitrogen spin-lattice relaxation dominates in the solution with FN-2a. These measurements provide a direct evidence for the two different mechanisms involved in the saturation behaviour of the nitroxide EPR line in the investigated concentration regime.

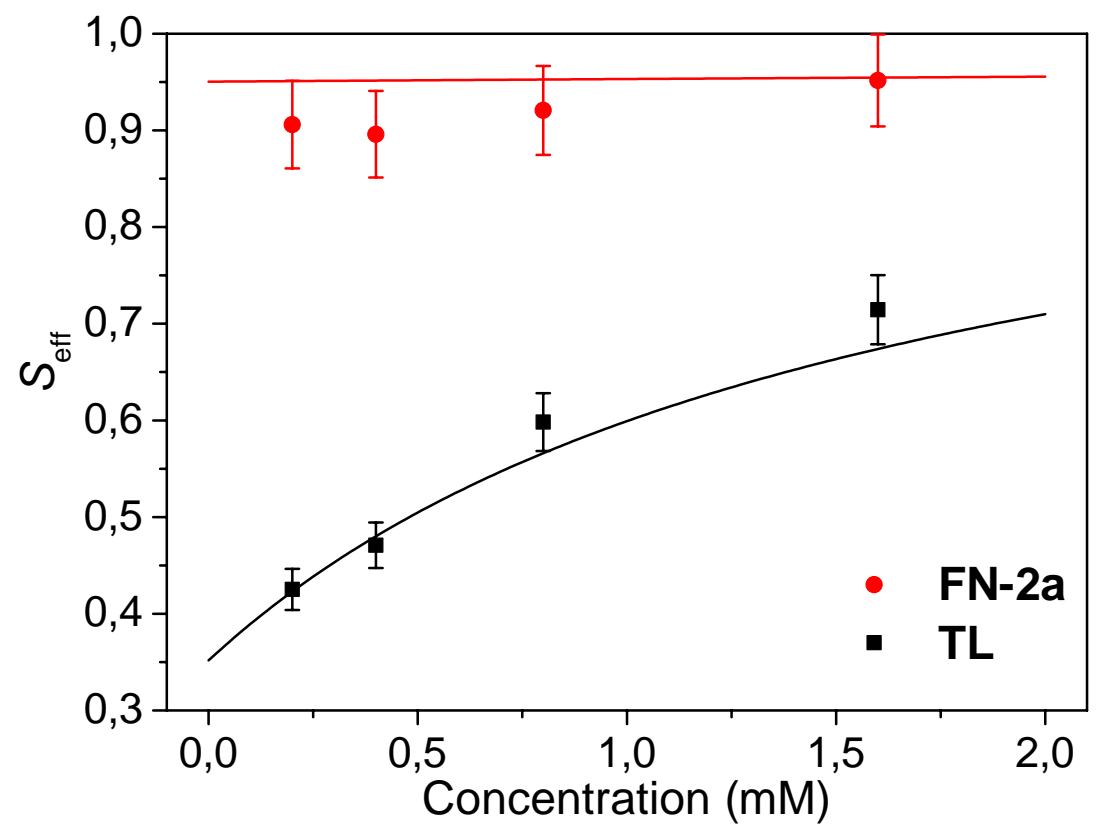

Figure 5.6: The polarizer concentration dependence of the effective saturation factor when the central EPR transition is irradiated. The experimental values (red circles and black squares) are from the ELDOR experiments. The solid lines are theoretically simulated curves using the rate parameters obtained from fitting polarization recovery curves.

Thus, fast nuclear spin-lattice relaxation leads to a high saturation factor in DNP. Unlike the Heisenberg spin exchange, the nuclear relaxation does not depend on the polarizer concentration and makes it possible to achieve high DNP enhancements at a relatively low concentration of a polarizing agent $(\sim 1 \mathrm{mM})$. The hypothesis about the nuclear relaxation effect on the saturation factor was previously considered by Armstrong and Han, and applied to tethered nitroxide radicals ${ }^{72,136}$. The present results indicate that 


\section{DNP with fullerene-nitroxide derivatives: effect of polarizer's size}

the correlation time dependence of the saturation factor is quite dramatic and it should be considered in investigations of new polarizers.

5.1.5. Coupling factor. Using experimentally determined DNP parameters $\varepsilon, f$ and $s$ (Table 5.2) the coupling factors $\xi$ were calculated for all investigated samples according to Overhauser equation (2.9). There is some but not substantial decrease of the coupling factor $\xi$ between TL and FN-3a $(\triangle \xi \approx 25 \%)$ (Table 5.2). This behavior is consistent with the current mechanistic model for DNP in toluene with nitroxide radical, which is governed by dipolar relaxation driven by molecular translational diffusion and the accessibility to the nitroxide electron spin center ${ }^{81,136,224}$. When the molecular size of a polarizing agent increases it leads to an increase of the translational diffusional correlation time

$$
\tau_{D}=\frac{d^{2}}{D_{S}+D_{P}},
$$

where $d$ is the distance of closest approach between electron and nuclear spins, $D_{S}$ and $D_{P}$ are the diffusion coefficients of the solvent and polarizer, respectively ${ }^{81}$. The increase of $\tau_{D}$ finally leads to a decrease in the coupling factor. The increases of the saturation $s$ and leakage $f$ factors largely compensate the decrease in the coupling factor $\boldsymbol{\xi}$ and lead to high DNP enhancement. 
Table 5.2: Summary of DNP parameters, relaxation times and Heisenberg exchange rate constants for toluene solutions of TL and FN derivatives. In DNP, microwave irradiation was set on the central hf line. Errors in $T_{1 \mathrm{e}}, T_{1 \mathrm{n}}, K_{\mathrm{x}}$ from fits of the polarization recovery data as well as $\tau_{c}$ are estimated up to $20 \%$. Errors in $\varepsilon$ (from DNP) are about $5 \%$, whereas the errors in $f$ and $s_{\text {eff }}$ are $\leq 5 \%$. The resulting error in $\xi$ is about $\pm 10 \%$. However, errors in the trends among the samples are much less, as pointed out previously ${ }^{227}$.

\begin{tabular}{|c|c|c|c|c|c|c|c|c|c|c|c|}
\hline \multirow{2}{*}{ Sample } & \multicolumn{2}{|c|}{$\varepsilon$} & \multicolumn{2}{|c|}{$f$} & \multirow{2}{*}{$s_{e f f}$} & \multicolumn{2}{|c|}{$\xi$} & \multirow{2}{*}{$\begin{array}{c}\tau_{c} \\
(\mathbf{n s})\end{array}$} & \multirow{2}{*}{$\begin{array}{l}T_{1 \mathrm{e}} \\
(\mathrm{ns})\end{array}$} & \multirow{2}{*}{$\frac{\boldsymbol{T}_{\mathbf{1 n}}(\mathbf{n s})}{{ }^{14} \mathrm{~N}}$} & \multirow{2}{*}{$\begin{array}{c}K_{\mathbf{x}} \\
\left(\mathbf{n s}^{-1} \mathbf{M}^{-1}\right)\end{array}$} \\
\hline & $\mathrm{H}_{\text {ring }}$ & $\mathrm{H}_{\text {methyl }}$ & $\mathrm{H}_{\text {ring }}$ & $\mathrm{H}_{\text {methyl }}$ & & $\mathrm{H}_{\text {ring }}$ & $\mathrm{H}_{\text {methyl }}$ & & & & \\
\hline TL & 94 & 64 & 0.75 & 0.58 & 0.71 & 0.27 & 0.24 & 0.01 & 400 & $\begin{array}{c}\geq \\
1.7 \times 10^{3}\end{array}$ & 5.1 \\
\hline FN & 103 & 73 & 0.8 & 0.68 & 0.77 & 0.26 & 0.21 & 0.06 & 1100 & $\geq 1 \times 10^{3}$ & 2.6 \\
\hline FN-1a & 111 & 83 & 0.82 & 0.7 & 0.89 & 0.23 & 0.20 & 0.15 & 2600 & 340 & 1.4 \\
\hline FN-2a & 110 & 87 & 0.83 & 0.71 & 0.95 & 0.21 & 0.20 & 0.30 & 3700 & 150 & 0.6 \\
\hline FN-3a & 107 & 78 & 0.85 & 0.75 & 0.95 & 0.20 & 0.17 & 0.45 & 4000 & 70 & $\leq 0.6$ \\
\hline
\end{tabular}

\subsection{Conclusion}

The experimental and theoretical analyses of the investigated samples have demonstrated several phenomena which clarify the design of new polarizers for DNP in liquids. The increase of the polarizer size leads to higher DNP efficiency because of the effective saturation factor increasing despite of simultaneous decrease of the coupling factor. The occurrence of fast nuclear relaxation in nitroxide radicals allows for achieving a high effective saturation factor even at low polarizer concentration because of its independence on radical concentration. It is possible to functionalize nitroxide radicals and maintain their DNP efficiency. 


\section{DEP with fullerene-nitroxide derivatives}

\section{DEP with fullerene-nitroxide derivatives}

\subsection{Transient EPR of fullerene-nitroxide derivatives}

The EPR transitions of the excited states, which can be detected in the liquid state at room temperature and at $0.35 \mathrm{~T}$, are the doublet states $\mathbf{D}_{\mathbf{S 0}}$ and $\mathbf{D}_{\mathbf{T 1}}$ and a transition $-1 / 2 \leftrightarrow+1 / 2$ inside the quartet $\mathbf{Q}_{\mathrm{T} 1}{ }^{159,229}$. The hyperfine interaction with ${ }^{14} \mathrm{~N}$ nucleus $(I=1)$ is revealed by three equally separated EPR transitions. The mixture of all these independent EPR transitions is displayed as five lines (Fig. 6.1). The g-factor and hyperfine splitting of the $\mathbf{D}_{\mathbf{T} 1}$ ( $g_{D T}$ and $A_{D T}$, respectively) and the $\mathbf{Q}_{\mathbf{T} 1}\left(g_{Q T}\right.$ and $A_{Q T}$, respectively) can be expressed as ${ }^{177}$

$$
\begin{array}{ll}
g_{D T}=-\frac{1}{3} g_{R}+\frac{4}{3} g_{T} & A_{D T}=-\frac{1}{3} A_{R} \\
g_{Q T}=\frac{1}{3} g_{R}+\frac{2}{3} g_{T} & A_{Q T}=\frac{1}{3} A_{R}
\end{array},
$$

where $g_{R}$ and $A_{R}$ are g-factor and hyperfine splitting, respectively, of the nitroxide radical (TEMPO), $g_{T}$ is $\mathrm{g}$-factor of fullerene $\left(\mathrm{C}_{60}\right)$ triplet state. Fig. $\mathbf{6 . 1}$ shows the distribution of the hyperfine EPR transitions of the electron spin states $\mathbf{D}_{\mathbf{S} \mathbf{0}}, \mathbf{D}_{\mathbf{T} 1}$ and $\mathbf{Q}_{\mathbf{T} 1}$. 


\section{DEP with fullerene-nitroxide derivatives}

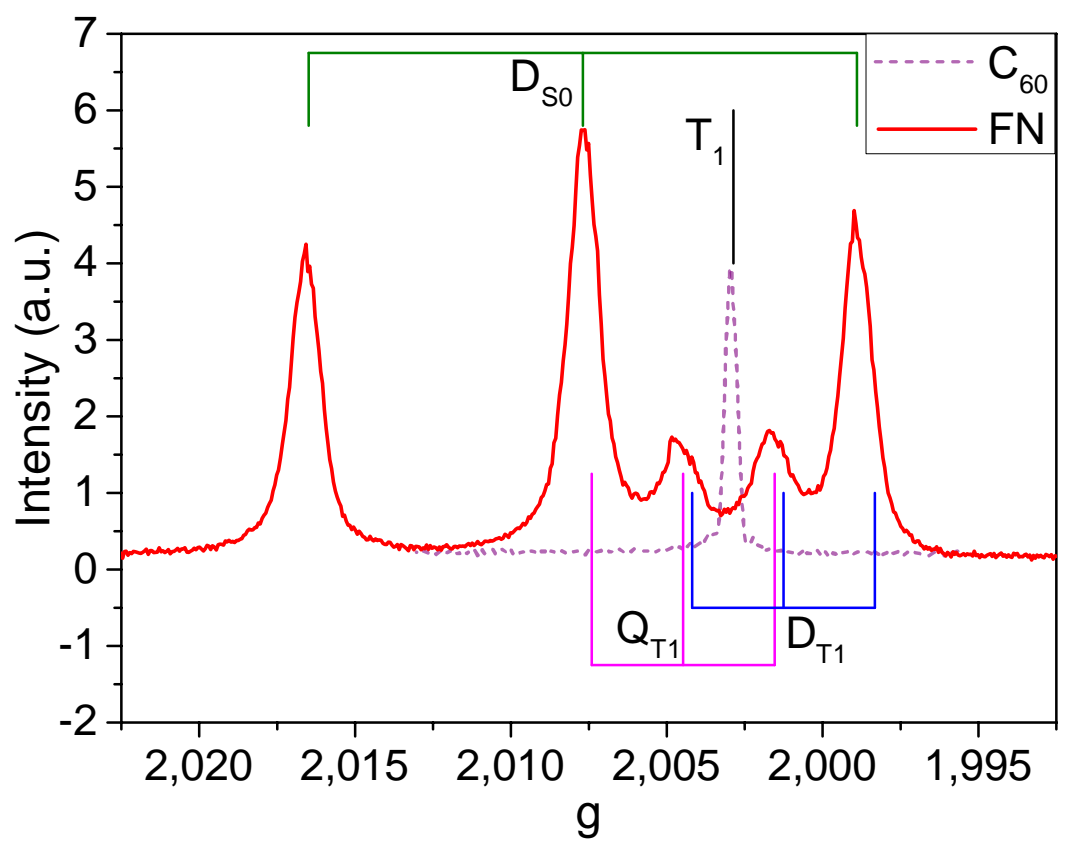

Figure 6.1: TrEPR spectra of photoexcited FN (red) and $\mathrm{C}_{60}$ triplet state (light purple). $g_{R} \approx 2.0077, A_{R} \approx 15.25 \mathrm{G}$ and $g_{T} \approx 2.0029$.

The TrEPR spectra of all investigated samples (Chapter 3) in the photoexcited state in the degassed toluene solutions consist of five lines (Fig. 6.2). As it is clear the low field EPR line corresponds to the $\mathbf{D}_{\mathbf{S} 0}$ transition only, the second one is a mixture of the $\mathbf{D}_{\mathbf{S o}}$ and $\mathbf{Q}_{\mathbf{T} 1}$ EPR lines and the high filed EPR line is a combination of ground state $\mathbf{D}_{\mathbf{S} 0}$ and the doublet $\mathbf{D}_{\mathbf{T} 1}$ transitions. The $3^{\text {rd }}$ and $4^{\text {th }}$ EPR lines are sum of the $\mathbf{D}_{\mathbf{T} 1}$ and $\mathbf{Q}_{\mathbf{T} 1}$ ones. 


\section{DEP with fullerene-nitroxide derivatives}
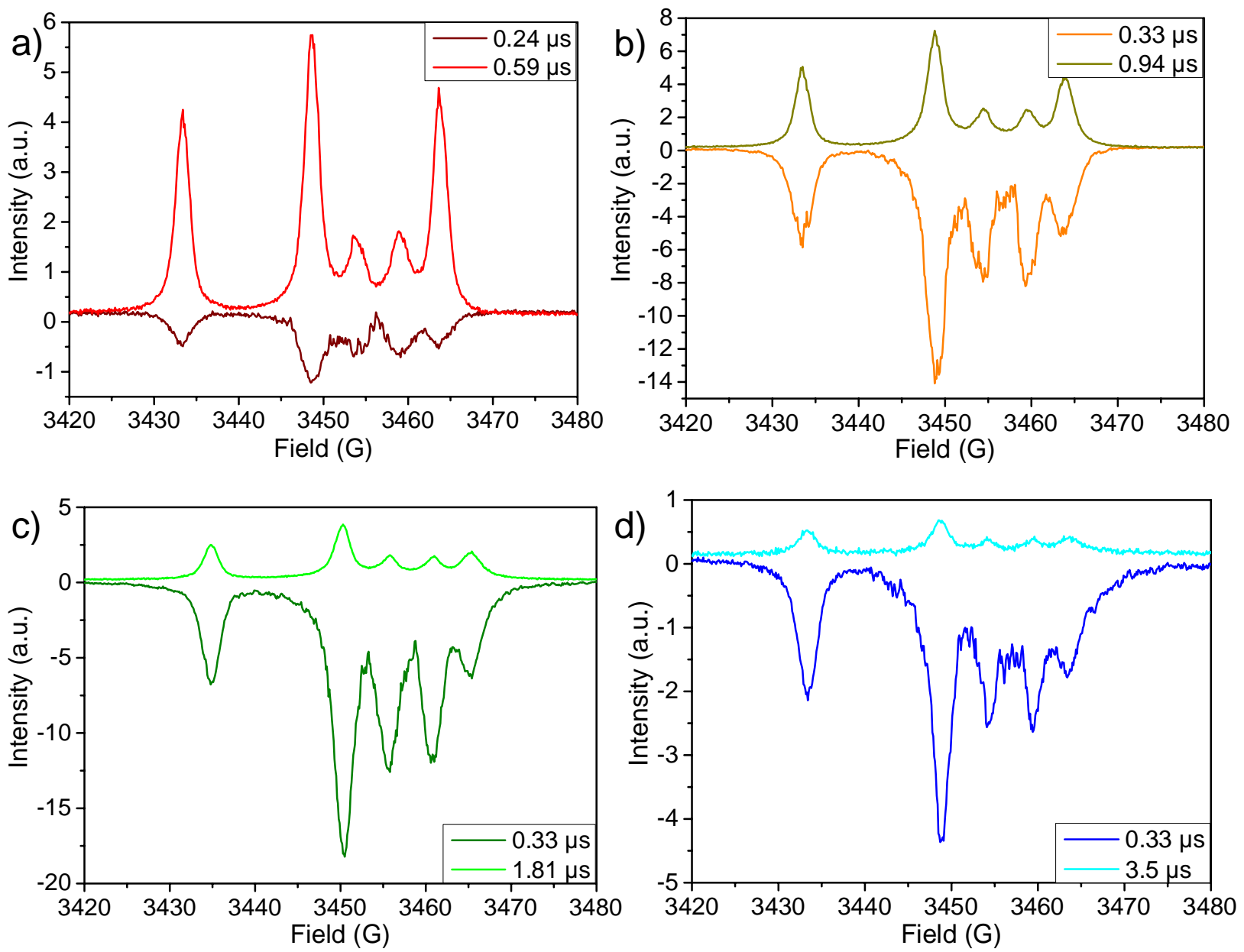

Figure 6.2: TrEPR spectra of FN (a), FN-1a (b), FN-2a (c), FN-3a (d) recorded at the described time delay after a laser flash (insert).

The time evolutions of the EPR signals after the laser pulse start from emission and are then sequentially reversed to absorption (Fig. 6.3). It is found that the amplitude of emissive part increases whereas the amplitude of absorption decreases with the number of adducts in the investigated molecules (from $\mathbf{F N}$ to $\mathbf{F N - 3 a ) . ~}$ 


\section{DEP with fullerene-nitroxide derivatives}
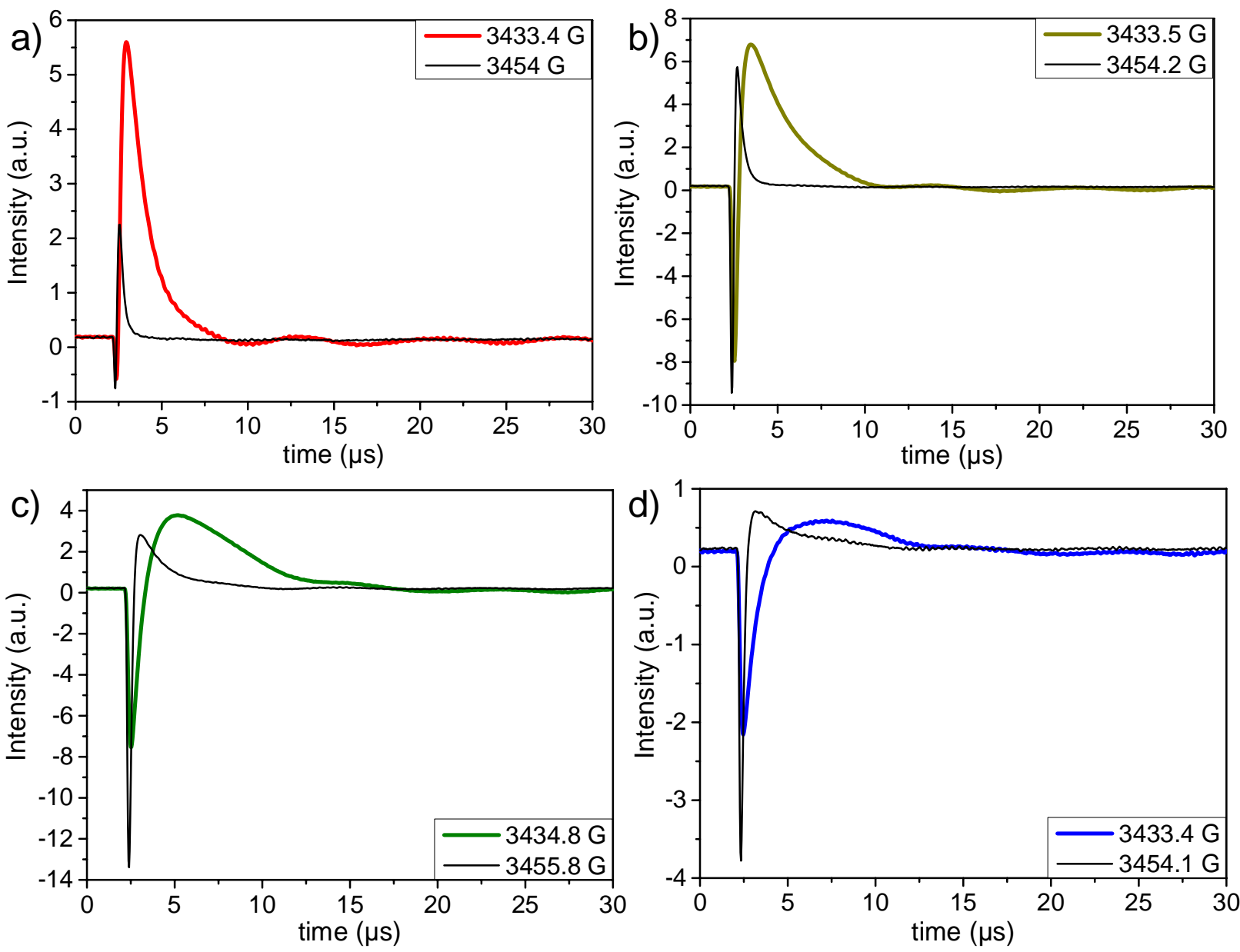

Figure 6.3: Time evolution of EPR signals of $1^{\text {st }}$ (color) and $3^{\text {rd }}$ (black) lines for FN (a), FN-1a (b), FN-2a (c) and FN-3a (d).

According to the model described by Corvaja (Chapter 2), in all observed systems the exchange coupling between the triplet and the radical have positive sign $\left(J_{R T}>0\right)$. Under the theoretical model of the reversed quartet mechanism (RQM) ${ }^{154}$ (Chapter 3), the phenomena of the "absorption-to-emission" ration changing can be conditioned mainly by different initial polarizations of $\mathbf{D}_{\mathbf{T} 1}$ and $\mathbf{Q}_{\mathbf{T} 1}$ and the ISC transition rates between the latter states. However, it requires more detail analysis of the dynamics inside the radicaldye systems. Also, it is not clear yet how the molecular size/correlation time affects the behavior. 


\section{DEP with fullerene-nitroxide derivatives}

\subsection{Perspectives of DNP with photoexcited fullerene-nitroxide systems}

6.2.1. Excitation efficiency. Since the CW transient EPR technique detects just a polarization change and the steady state (thermal equilibrium/Boltzmann) signal cannot be detected by this method, pulsed time-resolved EPR was applied to measure the excitation efficiency $(\alpha)$ and the electron polarization enhancement $\left(\varepsilon_{D E P}=P_{D E P} / P_{\text {Boltzmann }}\right)($ Fig. 6.4).

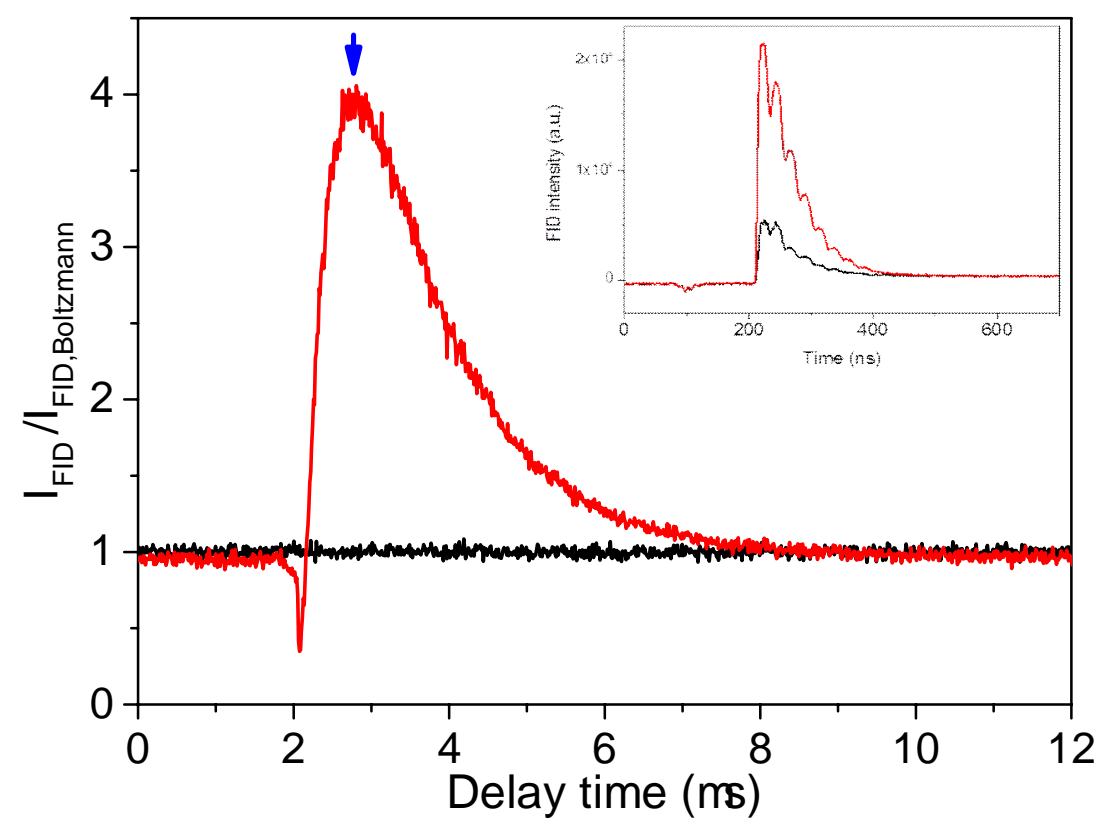

Figure 6.4: The normalized low field line FID intensity $\left(I_{F I D} / I_{F I D, B o l t z m a n n}\right)$ of $\mathbf{F N}$ in toluene as a function of the delay time for photoexcited (red) and Boltzmann (white) states. Insert: the FID traces measured at the delay time marked by a blue arrow. Experimental details: sample concentration $\sim 0.8 \mathrm{mM}$, volume $\sim 20 \mu \mathrm{L}, \mathrm{MW}$ “ $\pi / 2$ ” length $20 \mathrm{~ns}$.

To estimate the fractions $\alpha$ in fullerene-nitroxide derivatives, which depend on quantum yield, fraction of excited volume and etc., pure fullerene $\mathrm{C}_{60}$ was used as reference sample to calibrate the experimental factors related to laser excitation (Fig.6.5). Fullerene triplet state has long electron spin-spin relaxation time $\left(T_{2} \approx 430 \mathrm{~ns}\right)$ and can be detected at room temperature in solution by pulse EPR. To quantify the number of spins in pulse EPR, the dead time effect in FID should be considered (Fig.3.8)

$$
I^{(\mathrm{det})}=e^{-t_{d} / T_{2}}
$$

where $t_{d}$ is the deadtime, $I^{(d e t)}$ is a detected FID intensity. 


\section{DEP with fullerene-nitroxide derivatives}

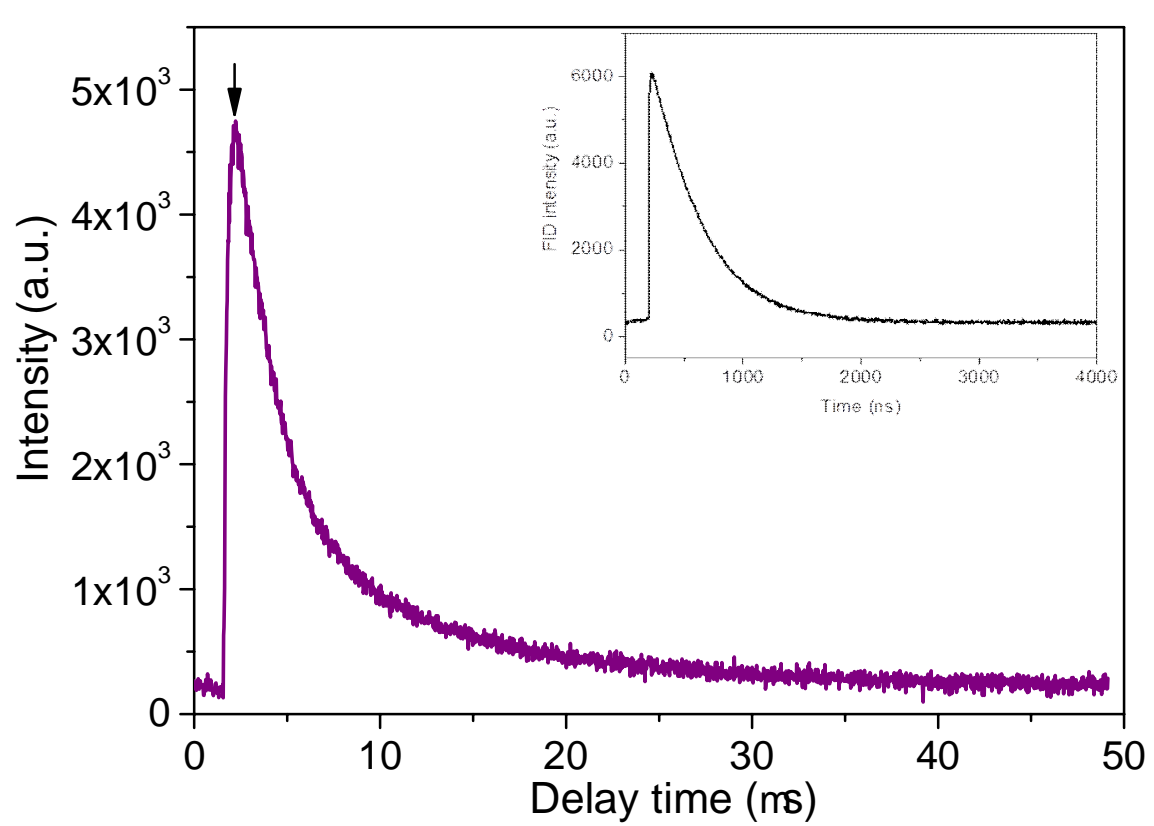

Figure 6.5: Kinetic trace of the photoexcited triplet state of $\mathrm{C}_{60}$ in toluene solution $(1.5 \mathrm{mM}$, $20 \mu \mathrm{L}$ ) by pulsed time-resolved EPR. Insert: FID decay of the peak of ${ }^{3} \mathrm{C}_{60}$ signal (black arrow).

After the calibration of concentration dependence of FID intensity with a nitroxide radical (TEMPONE- $\mathrm{D}_{16^{-}}{ }^{15} \mathrm{~N}$ ) at fixed experimental conditions the fraction of the excited triplet state can be given by

$$
\begin{gathered}
I_{e x c}^{C 60}=\alpha \cdot N \cdot P_{T 1}, I_{N O^{\bullet}}=\frac{1}{2} N \cdot P_{N O^{\bullet}} \\
\alpha=\frac{1}{2}\left(\frac{I_{e x c}^{C 60}}{I_{N O^{\bullet}}} / \frac{P_{T 1}}{P_{N O^{\bullet}}}\right),
\end{gathered}
$$

where $P_{T 1}$ and $P_{N O}$ are electron spin polarizations of the excited triplet state of fullerene and the thermal equilibrium state, respectively, at the same sample concentration. The factor $1 / 2$ is used because TEMPONE- $\mathrm{D}_{16^{-}}{ }^{15} \mathrm{~N}$ has two equivalent hyperfine EPR lines. The excited triplet of $\mathrm{C}_{60}$ has approximately equal population of the states $|1 ;-1\rangle$ and $|1 ;+1\rangle$ at the initial time point after its formation and evolves towards the Boltzmann distribution through relaxation ${ }^{174,230}$. Since the Boltzmann polarization of a spin system with $S=1$ is equals to eight third of a polarization of a spin system with $S=1 / 2$ the final expression for $\alpha$ parameter is 174,230

$$
P_{T 1}=\frac{8}{3} P_{N O^{\circ}} \Rightarrow \alpha=\frac{3}{16} \frac{I_{e x c}^{C 60}}{I_{N O^{\circ}}} .
$$




\section{DEP with fullerene-nitroxide derivatives}

The value of $\alpha$ in the case of $0.8 \mathrm{mM} \mathrm{C}_{60}$ with the $t_{d}$ values $200 \mathrm{~ns}$ is $0.10 \pm 0.03$.

Another way to estimate the fraction $\alpha$ is from photon absorption spectra (Fig.3.4). Beer-Lambert law relates the attenuation of light intensity during the light traveling through any material ${ }^{176}$

$$
I_{\text {trans }} / I_{0}=e^{-\sigma_{g} \cdot N_{A} \cdot n \cdot l}=10^{-\varepsilon \cdot n \cdot l},
$$

where $I_{0}$ and $I_{\text {trans }}$ are the intensities of initially incident and transmitted light, respectively; $\varepsilon$ and $\sigma_{g}$ are the molar attenuation coefficient and attenuation cross section of the material, respectively; $n$ is the sample concentration, $l$ is the path length of the light beam through the sample; $N_{A}$ is Avogadro constant. Due to small size of the tube and small concentration of sample, it can be simplified

$$
\begin{gathered}
I_{\text {trans }} / I_{0}=\left(N_{0}-N_{a b s}\right) / N_{0} \approx 1-\sigma_{g} \cdot N_{A} \cdot n \cdot l=1-\varepsilon \cdot n \cdot l \cdot \ln 10 \\
N_{a b s} / N_{0} \approx \sigma_{g} \cdot N_{A} \cdot n \cdot l=\sigma_{g} \cdot N_{V} \cdot l=\varepsilon \cdot n \cdot l \cdot \ln 10,
\end{gathered}
$$

where $N_{0}$ and $N_{a b s}$ are the number of initially received and absorbed photons, respectively, $N_{V}$ is the density of the material (number of molecules per volume). The ratio of a number of excited molecules to a number of absorbed photons is the quantum yield of the excited state $\left(Y_{e x c}\right)$. Thus, the fraction can be calculated by

$$
\alpha=Y_{\text {exc }} \cdot \frac{\sigma_{g}}{\sigma} \cdot \frac{E_{p}}{\mathrm{~h} \omega}=Y_{e x c} \cdot \frac{\varepsilon}{N_{A} \cdot \sigma} \cdot \frac{E_{p}}{\mathrm{~h} \omega} \cdot \ln 10,
$$

where $\sigma$ is a section of the laser beam, $E_{p}$ is the energy of single laser pulse. Substituting the characteristic parameters experimental setup of $\mathrm{C}_{60}\left(E_{p}=5 \mathrm{~mJ}, \lambda=2 \pi \cdot c / \omega=532 \mathrm{~nm}\right.$, $\left.\sigma \approx 3.98 \mathrm{~mm}^{2}, \varepsilon \sim 1 \mathrm{mM}^{-1} \cdot \mathrm{cm}^{-1}, Y_{\text {exc }} \approx 1\right)$ in the equation (6.7) we obtain $\alpha \approx 0.24$. Considering the presence of the grid in the optical window of the resonator for laser beam (the additional light loss $\sim 20 \%$ ) the calculated value of the fraction $\alpha$ is $\approx 0.2$. The difference between the calculated and values is around $30 \%$. The calculated value is over estimated because the maximum initial light intensity was used in the equation (6.7) whereas the intensity is actually decreasing over path length $l$. 


\section{DEP with fullerene-nitroxide derivatives}

6.2.2. Electron spin polarization. In pulse EPR, the signal intensity is the sum of signals from all molecules but not just the molecules in excited states after light irradiation. So the effective enhancement as ratio of the signal intensities of the sample with excitation and in thermal equilibrium states $\left(I_{\text {exc }} / I_{\text {Boltzmann }}\right)$ is less than $\varepsilon_{D E P}$. Thus, knowing the fraction of the excited molecules under the experimental conditions, the DEP enhancement can be estimated by

$$
\begin{aligned}
& \frac{I_{\text {exc }}}{I_{\text {Boltzmann }}}=\frac{\alpha \cdot P_{D E P}+(1-\alpha) \cdot P_{\text {Boltzmann }}}{P_{\text {Boltzmann }}}=\alpha \cdot \varepsilon_{D E P}+(1-\alpha) \\
& \varepsilon_{D E P}=\frac{1}{\alpha} \cdot\left(\frac{I_{\text {exc }}}{I_{\text {Boltzmann }}}-1+\alpha\right)=\frac{1}{\alpha} \cdot\left(\frac{I_{\text {exc }}}{I_{\text {Boltzmann }}}-1\right)+1
\end{aligned} .
$$

The measured ratio of absorption and Boltzmann signal intensities is 4 for low field EPR line of FN (Fig. 6.4). The emissive signal intensity is 0.25 of the Boltzmann one. Using the calculated fraction value for $\mathbf{F N}(\alpha \approx 0.18 \pm 0.05)$, the assuming $Y_{e x c} \approx 1$ and the equation $(6.8)$ there has been obtained $\varepsilon_{D E P} \approx 18 \pm 9$.

\subsection{Conclusion}

The availability of high non-equilibrium electron spin polarization, generated by light excitation, of the paramagnetic moiety make it is possible to overcome the limit of the deviation/saturation factor which cannot be larger than 1 for Overhauser DNP with MW saturation. If the electron spin polarization achieved in FN can be efficiently transferred to nuclei within the electron relaxation time (several microseconds), e.g. through pulse DNP, the deviation factor $\left(s=1-\varepsilon_{D E P}\right)$ can be 3 times larger than in the DNP experiment with MW irradiation. This number can be even greater and reach around 20 in the ideal case when the FN sample is completely excited $(\alpha=1)$.

To keep high non-equilibrium electron polarization of fullerene-nitroxide derivatives, which lifetimes are limited by the electron relaxation times, continuous light excitation is required. In the case of excitation efficiency of $5 \%(\alpha=0.05)$ for $\mathbf{F N}$, the deviation of 
electron spin polarization from Boltzman value is approximately the same as in Overhauser DNP with MW saturation. To reach this efficiency for pure $\mathrm{C}_{60}$ the laser power of $8 \mathrm{~W}$ (at $355 \mathrm{~nm}$ ) is required (Fig. 2.8). This power is still high for applications of DNP with radical-dye photopolarizer. The simultaneous cooling during the laser excitation can reduced the heating of a system under study. The further detailed studies of the investigated systems should be resumed to overcome the disadvantages. 


\section{References}

\section{References}

1. Overhauser, A. W., Polarization of Nuclei in Metals. Phys. Rev. 1953, 92, (2), 411-415.

2. Overhauser, A. W., Polarization of Nuclei in Metals. Phys. Rev. 1953, 91, (2), 476-476.

3. Carver, T. R.; Slichter, C. P., Polarization of Nuclear Spins in Metals. Phys. Rev. 1953, 92, (1), 212-213.

4. Carver, T. R.; Slichter, C. P., Experimental Verification of the Overhauser Nuclear Polarization Effect. Phys. Rev. 1956, 102, (4), 975-980.

5. Abragam, A., Overhauser Effect in Nonmetals. Phys. Rev. 1955, 98, (6), 1729-1735.

6. Bennett, L. H.; Torrey, H. C., High Negative Nuclear Polarizations in a Liquid. Phys. Rev. 1957, 108, (2), 499-500.

7. Hausser, K. H.; Stehlik, D., Dynamic Nuclear Polarization in Liquids. In Advances in Magnetic and Optical Resonance, John S, W., Ed. Academic Press: 1968; Vol. 3, pp 79139.

8. Kessenikh, A. V.; Lushchikov, V. I.; Manenkov, A. A.; Taran, Y. V., Proton Polarization in Irradiated Polyethylenes. Sov Phys Sol State 1963, 5, (2), 321-329.

9. Kessenikh, A. V.; Manenkov, A. A.; Pyatnitskii, G. I., On Explanation of Experimental Data on Dynamic Polarization of Protons in Irradiated Polyethylenes. Sov Phys Sol State 1964, 6, (3), 641-643.

10. Hwang, C. F.; Hill, D. A., New Effect in Dynamic Polarization. Phys Rev Lett 1967, 18, (4), 110-112.

11. Hwang, C. F.; Hill, D. A., Phenomenological Model for New Effect in Dynamic Polarization. Phys Rev Lett 1967, 19, (18), 1011-1014.

12. Wollan, D. S., Dynamic Nuclear Polarization with an Inhomogeneously Broadened ESR Line .1. Theory. Phys Rev B 1976, 13, (9), 3671-3685.

13. Wollan, D. S., Dynamic Nuclear Polarization with an Inhomogeneously Broadened ESR Line .2. Experiment. Phys Rev B 1976, 13, (9), 3686-3696.

14. Borghini, M., Spin-Temperature Model of Nuclear Dynamic Polarization Using Free Radicals. Phys Rev Lett 1968, 20, (9), 419-421.

15. Borghini, M.; Scheffle.K, Experimental Evidence for Dynamic Nuclear Polarization by Cooling of Electron Spin-Spin Interactions. Phys Rev Lett 1971, 26, (22), 1362-1365.

16. Hall, D. A.; Maus, D. C.; Gerfen, G. J.; Inati, S. J.; Becerra, L. R.; Dahlquist, F. W.; Griffin, R. G., Polarization-enhanced NMR spectroscopy of biomolecules in frozen solution. Science 1997, 276, (5314), 930-932.

17. Bouchiat, M. A.; Carver, T. R.; Varnum, C. M., Nuclear Polarization in 3He Gas Induced by Optical Pumping and Dipolar Exchange. Phys Rev Lett 1960, 5, (8), 373-375.

18. DiMauro, L. F.; Wood, D. E.; Lloyd, R. V., Polarized Emission from Doublet State Organic Free Radicals. J Chem Phys 1983, 78, (10), 5920-5925. 


\section{References}

19. Lampel, G., Nuclear Dynamic Polarization by Optical Electronic Saturation and Optical Pumping in Semiconductors. Phys Rev Lett 1968, 20, (10), 491-493.

20. Zeng, X.; Wu, Z.; Call, T.; Miron, E.; Schreiber, D.; Happer, W., Experimental Determination of the Rate Constants for Spin Exchange between Optically Pumped K, $\mathrm{Rb}$, and $\mathrm{Cs}$ Atoms and 129Xe Nuclei in Alkali-Metal-Noble-Gas Vanderwaals Molecules. Phys Rev A 1985, 31, (1), 260-278.

21. Pavlovskaya, G. E.; Cleveland, Z. I.; Stupic, K. F.; Basaraba, R. J.; Meersmann, T., Hyperpolarized krypton-83 as a contrast agent for magnetic resonance imaging. $P$ Natl Acad Sci USA 2005, 102, (51), 18275-18279.

22. Bifone, A.; Song, Y. Q.; Seydoux, R.; Taylor, R. E.; Goodson, B. M.; Pietrass, T.; Budinger, T. F.; Navon, G.; Pines, A., NMR of laser-polarized xenon in human blood. $P$ Natl Acad Sci USA 1996, 93, (23), 12932-12936.

23. Albert, M. S.; Cates, G. D.; Driehuys, B.; Happer, W.; Saam, B.; Springer, C. S.; Wishnia, A., Biological Magnetic Resonance Imaging Using Laser Polarized 129Xe. Nature 1994, 370, (6486), 199-201.

24. Navon, G.; Song, Y. Q.; Room, T.; Appelt, S.; Taylor, R. E.; Pines, A., Enhancement of solution NMR and MRI with laser-polarized xenon. Science 1996, 271, (5257), 18481851.

25. Berthault, P.; Huber, G.; Desvaux, H., Biosensing using laser-polarized xenon NMR/MRI. Prog Nucl Mag Res Sp 2009, 55, (1), 35-60.

26. Pavlovskaya, G. E.; Cleveland, Z.; Horton-Garcia, C.; Stupic, K.; LeNoir, C.; Meersmann, T., Novel advances with hyperpolarized gases in magnetic resonance imaging. Abstr Pap Am Chem S 2005, 229, U720-U720.

27. Bargon, J.; Fischer, H.; Johnson, U., Nuclear Magnetic Resonance Emission Lines During Fast Radical Reactions. I. Recording methods and examples. Zeitschrift Fur Naturforschung. A, Astrophysik, Physik Und Physikalische Chemie 1967, A 22, (10), 1551-1555.

28. Ward, H. R.; Lawler, R. G., Nuclear Magnetic Resonance Emission and Enhanced Absorption in Rapid Organometallic Reactions. J Am Chem Soc 1967, 89, (21), 55185519.

29. Cocivera, M., Optically Induced Overhauser Effect in Solution. Nuclear Magnetic Resonance Emission. J Am Chem Soc 1968, 90, (12), 3261-3263.

30. Kaptein, R.; Dijkstra, K.; Nicolay, K., Laser Photo-CIDNP as a Surface Probe for Proteins in Solution. Nature 1978, 274, (5668), 293-294.

31. Hore, P. J.; Broadhurst, R. W., Photo-CIDNP of Biopolymers. Prog Nucl Mag Res Sp 1993, 25, 345-402.

32. Lyon, C. E.; Jones, J. A.; Redfield, C.; Dobson, C. M.; Hore, P. J., Two-dimensional $15 \mathrm{~N}-1 \mathrm{H}$ photo-CIDNP as a surface probe of native and partially structured proteins. $J \mathrm{Am}$ Chem Soc 1999, 121, (27), 6505-6506.

33. Maeda, K.; Lyon, C. E.; Lopez, J. J.; Cemazar, M.; Dobson, C. M.; Hore, P. J., Improved photo-CIDNP methods for studying protein structure and folding. J Biomol NMR 2000, $16,(3), 235-244$. 


\section{References}

34. Kuprov, I.; Hore, P. J., Chemically amplified 19F-1H nuclear Overhauser effects. J Magn Reson 2004, 168, (1), 1-7.

35. Bowers, C. R.; Weitekamp, D. P., Transformation of Symmetrization Order to NuclearSpin Magnetization by Chemical-Reaction and Nuclear Magnetic Resonance. Phys Rev Lett 1986, 57, (21), 2645-2648.

36. Bowers, C. R.; Weitekamp, D. P., Parahydrogen and synthesis allow dramatically enhanced nuclear alignment. J Am Chem Soc 1987, 109, (18), 5541-5542.

37. Natterer, J.; Bargon, J., Parahydrogen induced polarization. Prog Nucl Mag Res Sp 1997, 31, 293-315.

38. Duckett, S. B.; Sleigh, C. J., Applications of the parahydrogen phenomenon: A chemical perspective. Prog Nucl Mag Res Sp 1999, 34, (1), 71-92.

39. Adams, R. W.; Aguilar, J. A.; Atkinson, K. D.; Cowley, M. J.; Elliott, P. I. P.; Duckett, S. B.; Green, G. G. R.; Khazal, I. G.; Lopez-Serrano, J.; Williamson, D. C., Reversible Interactions with para-Hydrogen Enhance NMR Sensitivity by Polarization Transfer. Science 2009, 323, (5922), 1708-1711.

40. Abragam, A.; Proctor, W. G., Une Nouvelle Methode De Polarisation Dynamique Des Noyaux Atomiques Dans Les Solides. Cr Hebd Acad Sci 1958, 246, (15), 2253-2256.

41. Erb, E.; Motchane, J. L.; Uebersfeld, J., Effet De Polarisation Nucleaire Dans Les Liquides Et Les Gaz Adsorbes Sur Les Charbons. Cr Hebd Acad Sci 1958, 246, (14), 2121-2123.

42. Jeffries, C. D., Polarization of Nuclei by Resonance Saturation in Paramagnetic Crystals. Phys. Rev. 1957, 106, (1), 164-165.

43. Henstra, A.; Dirksen, P.; Wenckebach, W. T., Enhanced Dynamic Nuclear Polarization by the Integrated Solid Effect. Phys Lett A 1988, 134, (2), 134-136.

44. Henstra, A.; Lin, T. S.; Schmidt, J.; Wenckebach, W. T., High Dynamic Nuclear Polarization at Room Temperature. Chem Phys Lett 1990, 165, (1), 6-10.

45. Weis, V.; Bennati, M.; Rosay, M.; Griffin, R. G., Solid effect in the electron spin dressed state: A new approach for dynamic nuclear polarization. J Chem Phys 2000, 113, (16), 6795-6802.

46. Weis, V.; Griffin, R. G., Electron-nuclear cross polarization. Solid State Nucl Mag 2006, 29, (1-3), 66-78.

47. Brunner, H.; Fritsch, R. H.; Hausser, K. H., Cross Polarization in Electron Nuclear Double-Resonance by Satisfying the Hartmann-Hahn Condition. Z Naturforsch A 1987, 42, (12), 1456-1457.

48. Henstra, A.; Dirksen, P.; Schmidt, J.; Wenckebach, W. T., Nuclear Spin Orientation Via Electron Spin Locking (NOVEL). J Magn Reson 1988, 77, (2), 389-393.

49. Wind, R. A.; Duijvestijn, M. J.; Vanderlugt, C.; Manenschijn, A.; Vriend, J., Applications of Dynamic Nuclear Polarization in 13C NMR in Solids. Prog Nucl Mag Res Sp 1985, 17, 33-67.

50. Abragam, A.; Goldman, M., Principles of Dynamic Nuclear Polarization. Rep Prog Phys 1978, 41, (3), 395-467.

51. Farrar, C. T.; Hall, D. A.; Gerfen, G. J.; Inati, S. J.; Griffin, R. G., Mechanism of dynamic nuclear polarization in high magnetic fields. J Chem Phys 2001, 114, (11), 4922-4933. 


\section{References}

52. Hu, K. N.; Bajaj, V. S.; Rosay, M.; Griffin, R. G., High-frequency dynamic nuclear polarization using mixtures of TEMPO and trityl radicals. J Chem Phys 2007, 126, (4).

53. Emsley, L., Spin Diffusion for NMR Crystallography. In eMagRes, John Wiley \& Sons, Ltd: 2007.

54. Hovav, Y.; Feintuch, A.; Vega, S., Dynamic nuclear polarization assisted spin diffusion for the solid effect case. J Chem Phys 2011, 134, (7).

55. Hartmann, S. R.; Hahn, E. L., Nuclear Double Resonance in the Rotating Frame. Phys. Rev. 1962, 128, (5), 2042-2053.

56. Pines, A.; Waugh, J. S.; Gibby, M. G., Proton-Enhanced Nuclear Induction Spectroscopy. A Method for High Resolution NMR of Dilute Spins in Solids. J Chem Phys 1972, 56, (4), 1776-1777.

57. Conte, P.; Spaccini, R.; Piccolo, A., State of the art of CPMAS 13C-NMR spectroscopy applied to natural organic matter. Prog Nucl Mag Res Sp 2004, 44, (3-4), 215-223.

58. Mehring, M., Principles of High Resolution NMR in Solids. 2nd ed.; Springer-Verlag: Berlin, 1983.

59. Rizzato, R.; Kaminker, I.; Vega, S.; Bennati, M., Cross-polarisation edited ENDOR. Mol Phys 2013, 111, (18-19), 2809-2823.

60. van den Heuvel, D. J.; Henstra, A.; Lin, T. S.; Schmidt, J.; Wenckebach, W. T., Transient Oscillations in Pulsed Dynamic Nuclear Polarization. Chem Phys Lett 1992, 188, (3-4), 194-200.

61. Schmidt, J.; Vandenheuvel, D. J.; Henstra, A.; Lin, T. S.; Wenckebach, W. T., Nuclear Spin Orientation via Pulsed EPR and Optically Cooled Triplet Electron Spins. Pure Appl Chem 1992, 64, (6), 859-863.

62. Reynhardt, E. C.; High, G. L., Dynamic nuclear polarization of diamond. II. Nuclear orientation via electron spin-locking. J Chem Phys 1998, 109, (10), 4100-4107.

63. Takeda, K.; Takegoshi, K.; Terao, T., Dynamic nuclear polarization by photoexcitedtriplet electron spins in polycrystalline samples. Chem Phys Lett 2001, 345, (1-2), 166170.

64. Freed, J. H., New technologies in electron spin resonance. Annu Rev Phys Chem 2000, 51, 655-689.

65. Bajaj, V. S.; Farrar, C. T.; Hornstein, M. K.; Mastovsky, I.; Vieregg, J.; Bryant, J.; Elena, B.; Kreischer, K. E.; Temkin, R. J.; Griffin, R. G., Dynamic nuclear polarization at $9 \mathrm{~T}$ using a novel $250 \mathrm{GHz}$ gyrotron microwave source. J Magn Reson 2003, 160, (2), 85-90.

66. Loening, N. M.; Rosay, M.; Weis, V.; Griffin, R. G., Solution-state dynamic nuclear polarization at high magnetic field. J Am Chem Soc 2002, 124, (30), 8808-8809.

67. Denninger, G.; Stocklein, W.; Dormann, E.; Schwoerer, M., Overhauser Shift on Radical Cation Salts of Fluoranthene. Mol Cryst Liq Cryst 1985, 120, (1-4), 233-236.

68. Can, T. V.; Caporini, M. A.; Mentink-Vigier, F.; Corzilius, B.; Walish, J. J.; Rosay, M.; Maas, W. E.; Baldus, M.; Vega, S.; Swager, T. M.; Griffin, R. G., Overhauser effects in insulating solids. J Chem Phys 2014, 141, (6).

69. Solomon, I., Relaxation Processes in a System of Two Spins. Phys. Rev. 1955, 99, (2), 559-565.

70. Poole, C. P., Electron spin resonance. John Wiley \& Sons: New York, 1983. 


\section{References}

71. Bates, R. D.; Drozdoski, W. S., Use of Nitroxide Spin Labels in Studies of Solvent-Solute Interactions. J Chem Phys 1977, 67, (9), 4038-4044.

72. Armstrong, B. D.; Han, S., A new model for Overhauser enhanced nuclear magnetic resonance using nitroxide radicals. J Chem Phys 2007, 127, (10).

73. Turke, M. T.; Bennati, M., Saturation factor of nitroxide radicals in liquid DNP by pulsed ELDOR experiments. Phys Chem Chem Phys 2011, 13, (9), 3630-3633.

74. Abragam, A.; Pound, R. V., Influence of Electric and Magnetic Fields on Angular Correlations. Phys. Rev. 1953, 92, (4), 943-962.

75. Müller-Warmuth, W.; Meise-Gresch, K., Molecular Motions and Interactions as Studied by Dynamic Nuclear Polarization (DNP) in Free Radical Solutions. In Advances in Magnetic and Optical Resonance, John S, W., Ed. Academic Press: 1983; Vol. Volume 11, pp 1-45.

76. Hwang, L. P.; Freed, J. H., Dynamic Effects of Pair Correlation Functions on Spin Relaxation by Translational Diffusion in Liquids. J Chem Phys 1975, 63, (9), 4017-4025.

77. Bennati, M.; Tkach, I.; Turke, M. T., Dynamic nuclear polarization in liquids. In Electron Paramagnetic Resonance: Volume 22, The Royal Society of Chemistry: 2011; Vol. 22, pp 155-182.

78. Reese, M.; Turke, M. T.; Tkach, I.; Parigi, G.; Luchinat, C.; Marquardsen, T.; Tavernier, A.; Hofer, P.; Engelke, F.; Griesinger, C.; Bennati, M., 1H and 13C Dynamic Nuclear Polarization in Aqueous Solution with a Two-Field (0.35 T/14 T) Shuttle DNP Spectrometer. J Am Chem Soc 2009, 131, (42), 15086-15087.

79. Lingwood, M. D.; Han, S. G., Dynamic nuclear polarization of $13 \mathrm{C}$ in aqueous solutions under ambient conditions. J Magn Reson 2009, 201, (2), 137-145.

80. George, C.; Chandrakumar, N., Chemical-Shift-Resolved 19F NMR Spectroscopy between 13.5 and $135 \mathrm{MHz}$ : Overhauser-DNP-Enhanced Diagonal Suppressed Correlation Spectroscopy. Angewandte Chemie International Edition 2014, 53, (32), 8441-8444.

81. Bennati, M.; Luchinat, C.; Parigi, G.; Turke, M. T., Water 1H relaxation dispersion analysis on a nitroxide radical provides information on the maximal signal enhancement in Overhauser dynamic nuclear polarization experiments. Phys Chem Chem Phys 2010, 12 , (22), 5902-5910.

82. Sezer, D.; Prandolini, M. J.; Prisner, T. F., Dynamic nuclear polarization coupling factors calculated from molecular dynamics simulations of a nitroxide radical in water. Phys Chem Chem Phys 2009, 11, (31), 6626-6637.

83. Kryukov, E. V.; Pike, K. J.; Tam, T. K. Y.; Newton, M. E.; Smith, M. E.; Dupree, R., Determination of the temperature dependence of the dynamic nuclear polarisation enhancement of water protons at 3.4 Tesla. Phys Chem Chem Phys 2011, 13, (10), 43724380 .

84. Griesinger, C.; Bennati, M.; Vieth, H. M.; Luchinat, C.; Parigi, G.; Hofer, P.; Engelke, F.; Glaser, S. J.; Denysenkov, V.; Prisner, T. F., Dynamic nuclear polarization at high magnetic fields in liquids. Prog Nucl Mag Res Sp 2012, 64, 4-28. 


\section{References}

85. Dorn, H. C.; Wang, J.; Allen, L.; Sweeney, D.; Glass, T. E., Flow Dynamic Nuclear Polarization, a Novel Method for Enhancing NMR Signals in Flowing Fluids. J Magn Reson 1988, 79, (3), 404-412.

86. Dorn, H. C.; Gitti, R.; Tsai, K. H.; Glass, T. E., The Flow Transfer of a Bolus with 1H Dynamic Nuclear Polarization from Low to High Magnetic Fields. Chem Phys Lett 1989, $155,(2), 227-232$.

87. Stevenson, S.; Dorn, H. C., 13C Dynamic Nuclear Polarization: A Detector for Continuous-Flow, Online Chromatography. Anal Chem 1994, 66, (19), 2993-2999.

88. Stevenson, S.; Glass, T.; Dorn, H. C., 13C Dynamic Nuclear Polarization: an Alternative Detector for Recycled-Flow NMR Experiments. Anal Chem 1998, 70, (13), 2623-2628.

89. Purcell, E. M.; Pound, R. V., A Nuclear Spin System at Negative Temperature. Phys. Rev. 1951, 81, (2), 279-280.

90. Reese, M.; Lennartz, D.; Marquardsen, T.; Hofer, P.; Tavernier, A.; Carl, P.; Schippmann, T.; Bennati, M.; Carlomagno, T.; Engelke, F.; Griesinger, C., Construction of a liquid-state NMR DNP shuttle spectrometer: First experimental results and evaluation of optimal performance characteristics. Appl Magn Reson 2008, 34, (3-4), 301-311.

91. Krahn, A.; Lottmann, P.; Marquardsen, T.; Tavernier, A.; Turke, M. T.; Reese, M.; Leonov, A.; Bennati, M.; Hoefer, P.; Engelke, F.; Griesinger, C., Shuttle DNP spectrometer with a two-center magnet. Phys Chem Chem Phys 2010, 12, (22), 58305840 .

92. Lottmann, P.; Marquardsen, T.; Krahn, A.; Tavernier, A.; Hofer, P.; Bennati, M.; Engelke, F.; Griesinger, C., Evaluation of a Shuttle DNP Spectrometer by Calculating the Coupling and Global Enhancement Factors of L-Tryptophan. Appl Magn Reson 2012, 43, (1-2), 207-221.

93. Turke, M. T.; Tkach, I.; Reese, M.; Hofer, P.; Bennati, M., Optimization of dynamic nuclear polarization experiments in aqueous solution at $15 \mathrm{MHz} / 9.7 \mathrm{GHz}$ : a comparative study with DNP at 140 MHz/94 GHz. Phys Chem Chem Phys 2010, 12, (22), 5893-5901.

94. Hofer, P.; Parigi, G.; Luchinat, C.; Carl, P.; Guthausen, G.; Reese, M.; Carlomagno, T.; Griesinger, C.; Bennati, M., Field dependent dynamic nuclear polarization with radicals in aqueous solution. J Am Chem Soc 2008, 130, (11), 3254-3255.

95. Villanueva-Garibay, J. A.; Annino, G.; van Bentum, P. J. M.; Kentgens, A. P. M., Pushing the limit of liquid-state dynamic nuclear polarization at high field. Phys Chem Chem Phys 2010, 12, (22), 5846-5849.

96. Denysenkov, V. P.; Prandolini, M. J.; Krahn, A.; Gafurov, M.; Endeward, B.; Prisner, T. F., High-field DNP spectrometer for liquids. Appl Magn Reson 2008, 34, (3-4), 289-299.

97. Denysenkov, V.; Prandolini, M. J.; Gafurov, M.; Sezer, D.; Endeward, B.; Prisner, T. F., Liquid state DNP using a $260 \mathrm{GHz}$ high power gyrotron. Phys Chem Chem Phys 2010, 12, (22), 5786-5790.

98. Prandolini, M. J.; Denysenkov, V. P.; Gafurov, M.; Lyubenova, S.; Endeward, B.; Bennati, M.; Prisner, T. F., First DNP results from a liquid water-TEMPOL sample at $400 \mathrm{MHz}$ and $260 \mathrm{GHz}$. Appl Magn Reson 2008, 34, (3-4), 399-407. 


\section{References}

99. Prandolini, M. J.; Denysenkov, V. P.; Gafurov, M.; Endeward, B.; Prisner, T. F., HighField Dynamic Nuclear Polarization in Aqueous Solutions. J Am Chem Soc 2009, 131, (17), 6090-6092.

100. Kryukov, E. V.; Newton, M. E.; Pike, K. J.; Bolton, D. R.; Kowalczyk, R. M.; Howes, A. P.; Smith, M. E.; Dupree, R., DNP enhanced NMR using a high-power $94 \mathrm{GHz}$ microwave source: a study of the TEMPOL radical in toluene. Phys Chem Chem Phys 2010, 12, (22), 5757-5765.

101. Ardenkjaer-Larsen, J. H.; Fridlund, B.; Gram, A.; Hansson, G.; Hansson, L.; Lerche, M. H.; Servin, R.; Thaning, M.; Golman, K., Increase in signal-to-noise ratio of $>10,000$ times in liquid-state NMR. P Natl Acad Sci USA 2003, 100, (18), 10158-10163.

102. Kohler, S. J.; Yen, Y.; Wolber, J.; Chen, A. P.; Albers, M. J.; Bok, R.; Zhang, V.; Tropp, J.; Nelson, S.; Vigneron, D. B.; Kurhanewicz, J.; Hurd, R. E., In vivo 13 carbon metabolic imaging at 3T with hyperpolarized 13C-1-pyruvate. Magn Reson Med 2007, 58, (1), 65-69.

103. Kurhanewicz, J.; Bok, R.; Nelson, S. J.; Vigneron, D. B., Current and potential applications of clinical 13C MR spectroscopy. J Nucl Med 2008, 49, (3), 341-344.

104. Nelson, S. J.; Vigneron, D.; Kurhanewicz, J.; Chen, A.; Bok, R.; Hurd, R., DNPhyperpolarized 13C magnetic resonance metabolic imaging for cancer applications. Appl Magn Reson 2008, 34, (3-4), 533-544.

105. Park, I.; Larson, P. E. Z.; Zierhut, M. L.; Hu, S.; Bok, R.; Ozawa, T.; Kurhanewicz, J.; Vigneron, D. B.; VandenBerg, S. R.; James, C. D.; Nelson, S. J., Hyperpolarized 13C magnetic resonance metabolic imaging: application to brain tumors. Neuro-Oncology 2010, 12, (2), 133-144.

106. Kurhanewicz, J.; Vigneron, D. B.; Brindle, K.; Chekmenev, E. Y.; Comment, A.; Cunningham, C. H.; DeBerardinis, R. J.; Green, G. G.; Leach, M. O.; Rajan, S. S.; Rizi, R. R.; Ross, B. D.; Warren, W. S.; Malloy, C. R., Analysis of Cancer Metabolism by Imaging Hyperpolarized Nuclei: Prospects for Translation to Clinical Research. Neoplasia 2011, 13, (2), 81-97.

107. Denysenkov, V.; Prisner, T., Liquid state Dynamic Nuclear Polarization probe with Fabry-Perot resonator at 9.2 T. J Magn Reson 2012, 217, 1-5.

108. Jakdetchai, O.; Denysenkov, V.; Becker-Baldus, J.; Dutagaci, B.; Prisner, T. F.; Glaubitz, C., Dynamic Nuclear Polarization-Enhanced NMR on Aligned Lipid Bilayers at Ambient Temperature. J Am Chem Soc 2014, 136, (44), 15533-15536.

109. Ardenkjaer-Larsen, J. H.; Leach, A. M.; Clarke, N.; Urbahn, J.; Anderson, D.; Skloss, T. W., Dynamic Nuclear Polarization Polarizer for Sterile Use Intent. NMR Biomed 2011, 24, (8), 927-932.

110. Comment, A.; van den Brandt, B.; Uffmann, K.; Kurdzesau, F.; Jannin, S.; Konter, J. A.; Hautle, P.; Wenckebach, W. T. H.; Gruetter, R.; van der Klink, J. J., Design and performance of a DNP prepolarizer coupled to a rodent MRI scanner. Concept Magn Reson B 2007, 31B, (4), 255-269.

111. Batel, M.; Krajewski, M.; Weiss, K.; With, O.; Dapp, A.; Hunkeler, A.; Gimersky, M.; Pruessmann, K. P.; Boesiger, P.; Meier, B. H.; Kozerke, S.; Ernst, M., A multi-sample 94 


\section{References}

GHz dissolution dynamic-nuclear-polarization system. J Magn Reson 2012, 214, 166174.

112. Leggett, J.; Hunter, R.; Granwehr, J.; Panek, R.; Perez-Linde, A. J.; Horsewill, A. J.; McMaster, J.; Smith, G.; Kockenberger, W., A dedicated spectrometer for dissolution DNP NMR spectroscopy. Phys Chem Chem Phys 2010, 12, (22), 5883-5892.

113. Bowen, S.; Hilty, C., Rapid sample injection for hyperpolarized NMR spectroscopy. Phys Chem Chem Phys 2010, 12, (22), 5766-5770.

114. Liu, Y. P.; Villamena, F. A.; Rockenbauer, A.; Zweier, J. L., Trityl-nitroxide biradicals as unique molecular probes for the simultaneous measurement of redox status and oxygenation. Chem Commun 2010, 46, (4), 628-630.

115. Reynolds, S.; Patel, H., Monitoring the solid-state polarization of 13C, 15N, 2H, 29Si and 31P. Appl Magn Reson 2008, 34, (3-4), 495-508.

116. Wilson, D. M.; Hurd, R. E.; Keshari, K.; Van Criekinge, M.; Chen, A. P.; Nelson, S. J.; Vigneron, D. B.; Kurhanewicz, J., Generation of hyperpolarized substrates by secondary labeling with [1,1-13C] acetic anhydride. P Natl Acad Sci USA 2009, 106, (14), 55035507.

117. Vasos, P. R.; Comment, A.; Sarkar, R.; Ahuja, P.; Jannin, S.; Ansermet, J. P.; Konter, J. A.; Hautle, P.; van den Brandt, B.; Bodenhausen, G., Long-lived states to sustain hyperpolarized magnetization. P Natl Acad Sci USA 2009, 106, (44), 18469-18473.

118. Carravetta, M.; Johannessen, O. G.; Levitt, M. H., Beyond the T1 Limit: Singlet Nuclear Spin States in Low Magnetic Fields. Phys Rev Lett 2004, 92, (15), 153003.

119. Carravetta, M.; Levitt, M. H., Long-lived nuclear spin states in high-field solution NMR. J Am Chem Soc 2004, 126, (20), 6228-6229.

120. Pileio, G.; Carravetta, M.; Levitt, M. H., Storage of nuclear magnetization as long-lived singlet order in low magnetic field. P Natl Acad Sci USA 2010, 107, (40), 17135-17139.

121. Dutta, P.; Martinez, G.; Gillies, R., A new horizon of DNP technology: application to invivo 13C magnetic resonance spectroscopy and imaging. Biophys Rev 2013, 5, (3), 271281.

122. Gallagher, F. A.; Kettunen, M. I.; Day, S. E.; Hu, D. E.; Ardenkjaer-Larsen, J. H.; in't Zandt, R.; Jensen, P. R.; Karlsson, M.; Golman, K.; Lerche, M. H.; Brindle, K. M., Magnetic resonance imaging of $\mathrm{pH}$ in vivo using hyperpolarized 13C-labelled bicarbonate. Nature 2008, 453, (7197), 940-943.

123. Gallagher, F. A.; Kettunen, M. I.; Brindle, K. M., Imaging pH with hyperpolarized $13 \mathrm{C}$. NMR Biomed 2011, 24, (8), 1006-1015.

124. Bowen, S.; Sekar, G.; Hilty, C., Rapid determination of biosynthetic pathways using fractional isotope enrichment and high-resolution dynamic nuclear polarization enhanced NMR. NMR Biomed 2011, 24, (8), 1016-1022.

125. Bowen, S.; Hilty, C., Time-resolved dynamic nuclear polarization enhanced NMR spectroscopy. Angew Chem Int Edit 2008, 47, (28), 5235-5237.

126. Harris, T.; Giraudeau, P.; Frydman, L., Kinetics from Indirectly Detected Hyperpolarized NMR Spectroscopy by Using Spatially Selective Coherence Transfers. Chem Eur J 2011, 17, (2), 697-703. 


\section{References}

127. Jensen, P. R.; Meier, S.; Ardenkjaer-Larsen, J. H.; Duus, J. O.; Karlsson, M.; Lerche, M. H., Detection of low-populated reaction intermediates with hyperpolarized NMR. Chem Commun 2009, (34), 5168-5170.

128. Lerche, M. H.; Meier, S.; Jensen, P. R.; Baumann, H.; Petersen, B. O.; Karlsson, M.; Duus, J. O.; Ardenkjaer-Larsen, J. H., Study of molecular interactions with 13C DNPNMR. J Magn Reson 2010, 203, (1), 52-56.

129. Pavlova, A.; McCarney, E. R.; Peterson, D. W.; Dahlquist, F. W.; Lew, J.; Han, S., Sitespecific dynamic nuclear polarization of hydration water as a generally applicable approach to monitor protein aggregation. Phys Chem Chem Phys 2009, 11, (31), 68336839.

130. Kausik, R.; Srivastava, A.; Korevaar, P. A.; Stucky, G.; Waite, J. H.; Han, S., Local Water Dynamics in Coacervated Polyelectrolytes Monitored through Dynamic Nuclear Polarization-Enhanced 1H NMR. Macromolecules 2009, 42, (19), 7404-7412.

131. Ortony, J. H.; Cheng, C. Y.; Franck, J. M.; Kausik, R.; Pavlova, A.; Hunt, J.; Han, S. I., Probing the hydration water diffusion of macromolecular surfaces and interfaces. New $J$ Phys 2011, 13.

132. Armstrong, B. D.; Choi, J.; Lopez, C.; Wesener, D. A.; Hubbell, W.; Cavagnero, S.; Han, S., Site-Specific Hydration Dynamics in the Nonpolar Core of a Molten Globule by Dynamic Nuclear Polarization of Water. J Am Chem Soc 2011, 133, (15), 5987-5995.

133. Kausik, R.; Han, S., Dynamics and state of lipid bilayer-internal water unraveled with solution state $1 \mathrm{H}$ dynamic nuclear polarization. Phys Chem Chem Phys 2011, 13, (17), $7732-7746$.

134. Cheng, C. Y.; Wang, J. Y.; Kausik, R.; Lee, K. Y. C.; Han, S., An ultrasensitive tool exploiting hydration dynamics to decipher weak lipid membrane-polymer interactions. $J$ Magn Reson 2012, 215, 115-119.

135. Kausik, R.; Han, S., Ultrasensitive Detection of Interfacial Water Diffusion on Lipid Vesicle Surfaces at Molecular Length Scales. J Am Chem Soc 2009, 131, (51), 1825418256.

136. Franck, J. M.; Pavlova, A.; Scott, J. A.; Han, S., Quantitative cw Overhauser effect dynamic nuclear polarization for the analysis of local water dynamics. Prog Nucl Mag Res $S p$ 2013, 74, 33-56.

137. Doll, A.; Bordignon, E.; Joseph, B.; Tschaggelar, R.; Jeschke, G., Liquid state DNP for water accessibility measurements on spin-labeled membrane proteins at physiological temperatures. J Magn Reson 2012, 222, 34-43.

138. Valentine, K. G.; Mathies, G.; Bedard, S.; Nucci, N. V.; Dodevski, I.; Stetz, M. A.; Can, T. V.; Griffin, R. G.; Wand, A. J., Reverse Micelles As a Platform for Dynamic Nuclear Polarization in Solution NMR of Proteins. J Am Chem Soc 2014, 136, (7), 2800-2807.

139. Song, C. S.; Hu, K. N.; Joo, C. G.; Swager, T. M.; Griffin, R. G., TOTAPOL: A biradical polarizing agent for dynamic nuclear polarization experiments in aqueous media. $J \mathrm{Am}$ Chem Soc 2006, 128, (35), 11385-11390.

140. Matsuki, Y.; Maly, T.; Ouari, O.; Karoui, H.; Le Moigne, F.; Rizzato, E.; Lyubenova, S.; Herzfeld, J.; Prisner, T.; Tordo, P.; Griffin, R. G., Dynamic Nuclear Polarization with a Rigid Biradical. Angew Chem Int Edit 2009, 48, (27), 4996-5000. 


\section{References}

141. Ysacco, C.; Rizzato, E.; Virolleaud, M. A.; Karoui, H.; Rockenbauer, A.; Le Moigne, F.; Siri, D.; Ouari, O.; Griffin, R. G.; Tordo, P., Properties of dinitroxides for use in dynamic nuclear polarization (DNP). Phys Chem Chem Phys 2010, 12, (22), 5841-5845.

142. Ardenkjaer-Larsen, J. H.; Laursen, I.; Leunbach, I.; Ehnholm, G.; Wistrand, L. G.; Petersson, J. S.; Golman, K., EPR and DNP properties of certain novel single electron contrast agents intended for oximetric imaging. J Magn Reson 1998, 133, (1), 1-12.

143. Munnemann, K.; Bauer, C.; Schmiedeskamp, J.; Spiess, H. W.; Schreiber, W. G.; Hinderberger, D., A mobile DNP polarizer for clinical applications. Appl Magn Reson 2008, 34, (3-4), 321-330.

144. Dollmann, B. C.; Kleschyov, A. L.; Sen, V.; Golubev, V.; Schreiber, L. M.; Spiess, H. W.; Munnemann, K.; Hinderberger, D., Spin-Labeled Heparins as Polarizing Agents for Dynamic Nuclear Polarization. Chemphyschem 2010, 11, (17), 3656-3663.

145. Dollmann, B. C.; Junk, M. J. N.; Drechsler, M.; Spiess, H. W.; Hinderberger, D.; Munnemann, K., Thermoresponsive, spin-labeled hydrogels as separable DNP polarizing agents. Phys Chem Chem Phys 2010, 12, (22), 5879-5882.

146. Corzilius, B.; Smith, A. A.; Barnes, A. B.; Luchinat, C.; Bertini, I.; Griffin, R. G., HighField Dynamic Nuclear Polarization with High-Spin Transition Metal Ions. J Am Chem Soc 2011, 133, (15), 5648-5651.

147. Atkins, P. W.; Evans, G. T., Electron Spin Polarization in a Rotating Triplet. Mol Phys 1974, 27, (6), 1633-1644.

148. Wong, S. K.; Hutchins.Da; Wan, J. K. S., Chemically Induced Dynamic Electron Polarization. II. General Theory for Radicals Produced by Photochemical Reactions of Excited Triplet Carbonyl Compounds. J Chem Phys 1973, 58, (3), 985-989.

149. Adrian, F. J.; Bowers, V. A.; Cochran, E. L., ESR study of photolysis of n-butyl iodide: Secondary photolysis and structure of n-butyl radical. J Chem Phys 1975, 63, (2), 919923.

150. Wong, S. K.; Wan, J. K. S., Photochemically induced dynamic electron spin polarization. 1,4-Naphthosemiquinone radical in 2-propanol. J Am Chem Soc 1972, 94, (20), 71977198.

151. Adrian, F., Principles of the radical pair mechanism of chemically induced nuclear and electron spin polarization. Reviews of Chemical Intermediates 1979, 3, (1-2), 3-43.

152. Blattler, C.; Jent, F.; Paul, H., A novel radical-triplet pair mechanism for chemically induced electron polarization (CIDEP) of free radicals in solution. Chem Phys Lett 1990, $166,(4), 375-380$.

153. Fujisawa, J.; Ohba, Y.; Yamauchi, S., Electron-spin polarizations generated from interactions between excited triplet porphyrins and stable radicals studied by timeresolved electron paramagnetic resonance. J Phys Chem A 1997, 101, (4), 434-439.

154. Rozenshtein, V.; Berg, A.; Stavitski, E.; Levanon, H.; Franco, L.; Corvaja, C., Electron spin polarization of functionalized fullerenes. Reversed quartet mechanism. J Phys Chem A 2005, 109, (49), 11144-11154.

155. Rozenshtein, V.; Berg, A.; Levanon, H., Quartet Pair and Radical Quartet Pair Mechanisms of Electron Spin Polarization Developed within Nonbonded Dimers. Appl Magn Reson 2010, 37, (1-4), 567-580. 


\section{References}

156. Tateishi, K.; Negoro, M.; Kagawa, A.; Kitagawa, M., Dynamic Nuclear Polarization with Photoexcited Triplet Electrons in a Glassy Matrix. Angew Chem Int Edit 2013, 52, (50), 13307-13310.

157. Tateishi, K.; Negoro, M.; Nishida, S.; Kagawa, A.; Morita, Y.; Kitagawa, M., Room temperature hyperpolarization of nuclear spins in bulk. P Natl Acad Sci USA 2014, 111, (21), 7527-7530.

158. Atkins, P. W.; Evans, G. T., Chemically Induced Electron Spin Polarization: Rotating Triplet Model. Chem Phys Lett 1974, 25, (1), 108-110.

159. Corvaja, C.; Maggini, M.; Prato, M.; Scorrano, G.; Venzin, M., C60 Derivative Covalently Linked to a Nitroxide Radical: Time-Resolved EPR Evidence of Electron Spin Polarization by Intramolecular Radical-Triplet Pair Interaction. J Am Chem Soc 1995, 117, (34), 8857-8858.

160. Arena, F.; Bullo, F.; Conti, F.; Corvaja, C.; Maggini, M.; Prato, M.; Scorrano, G., Synthesis and EPR studies of radicals and biradical anions of C60 nitroxide derivatives. $J$ Am Chem Soc 1997, 119, (4), 789-795.

161. Moons, H.; Goovaerts, E.; Gubskaya, V. P.; Nuretdinov, I. A.; Corvaja, C.; Franco, L., W-band transient EPR and photoinduced absorption on spin-labeled fullerene derivatives. Phys Chem Chem Phys 2011, 13, (9), 3942-3951.

162. Conti, F.; Corvaja, C.; Gattazzo, C.; Toffoletti, A.; Bergo, P.; Maggini, M.; Scorrano, G.; Prato, M., Time-resolved EPR investigation of intramolecular photoinduced electron transfer in spin-labeled fullerene/ferrocene dyads. Phys Chem Chem Phys 2001, 3, (17), 3526-3531.

163. Enkin, N.; Liu, G.; Gimenez-Lopez, M. d. C.; Porfyrakis, K.; Tkach, I.; Bennati, M., A high saturation factor in Overhauser DNP with nitroxide derivatives: the role of $14 \mathrm{~N}$ nuclear spin relaxation. Phys Chem Chem Phys 2015, 17, 11144 - 11149.

164. Robinson, B. H.; Haas, D. A.; Mailer, C., Molecular dynamics in liquids: spin-lattice relaxation of nitroxide spin labels. Science 1994, 263, (5146), 490-493.

165. Hyde, J. S.; Chen, J. C. W.; Freed, J. H., Electron-Electron Double Resonance of Free Radicals in Solution. J Chem Phys 1968, 48, (9), 4211-4226.

166. Yin, J. J.; Pasenkiewiczgierula, M.; Hyde, J. S., Lateral Diffusion of Lipids in Membranes by Pulse Saturation Recovery Electron Spin Resonance. P Natl Acad Sci USA 1987, 84, (4), 964-968.

167. Bloembergen, N., Proton Relaxation Times in Paramagnetic Solutions. J Chem Phys 1957, 27, (2), 572-573.

168. Bertini, I.; Luchinat, C.; Parigi, G., 1H NMRD profiles of paramagnetic complexes and metalloproteins. Adv Inorg Chem 2005, 57, 105-172.

169. Ferrante, G.; Sykora, S., Technical aspects of fast field cycling. Adv Inorg Chem 2005, 57, 405-470.

170. Luchinat, C.; Parigi, G., Collective relaxation of protein protons at very low magnetic field: A new window on protein dynamics and aggregation. J Am Chem Soc 2007, 129, (5), 1055-1064. 


\section{References}

171. Conti, F.; Corvaja, C.; Maggini, M.; Piu, F.; Scorrano, G.; Toffoletti, A., Spin polarization and photoinduced electron transfer between ferrocene and fullerene derivatives containing a nitroxide group. Appl Magn Reson 1997, 13, (3-4), 337-346.

172. Corvaja, C.; Maggini, M.; Ruzzi, M.; Scorrano, G.; Toffoletti, A., Spin polarization in fullerene derivatives containing a nitroxide group. Observation of the intermediate photoexcited quartet state in radical triplet pair interaction. Appl Magn Reson 1997, 12, (4), 477-493.

173. Kawai, A.; Okutsu, T.; Obi, K., Spin polarization generated in the triplet-doublet interaction: hyperfine-dependent chemically induced dynamic electron polarization. $J$ Phys Chem 1991, 95, (23), 9130-9134.

174. Goudsmit, G. H.; Paul, H., Time-resolved EPR investigation of triplet state C60. Triplettriplet annihilation, CIDEP, and quenching by nitroxide radicals. Chem Phys Lett 1993, 208, (1-2), 73-78.

175. Kawai, A., Dynamic electron polarization created by the radical-triplet pair mechanism: Application to the studies on excited state deactivation processes by free radicals. Appl Magn Reson 2003, 23, (3-4), 349-367.

176. Turro, N. J., Modern Molecular Photochemistry. New edition ed.; University Science Books: Sausalito, 1991; p 628.

177. Bencini, A.; Gatteschi, D., Electron Paramagnetic Resonance of Exchange Coupled Systems. Springer-Verlag Berlin Heidelberg: Berlin, 1990; p 287.

178. Guldi, D. M.; Luo, C. P.; Da Ros, T.; Prato, M.; Dietel, E.; Hirsch, A., Photoinduced electron transfer in multicomponent arrays of a pi-stacked fullerene porphyrin dyad and diazabicyclooctane or a fulleropyrrolidine ligand. Chem Commun 2000, (5), 375-376.

179. Gouterman, M., 1 - Optical Spectra and Electronic Structure of Porphyrins and Related Rings. In The Porphyrins, Dolphin, D., Ed. Academic Press: 1978; Vol. 3, pp 1-165.

180. Naqvi, K. R., Spin selection rules concerning intermolecular energy transfer. Energytransfer studies using doublet-state acceptors. Comments. The Journal of Physical Chemistry 1981, 85, (15), 2303-2304.

181. Medvedev, E. S.; Osherov, V. I., Radiationless Transitions in Polyatomic Molecules. Springer: Berlin, 1995; p 374.

182. Devault, D., Quantum Mechanical Tunnelling in Biological Systems. Q Rev Biophys 1980, 13, (4), 387-564.

183. Samanta, A.; Kamat, P. V., Quenching of Fullerene Triplets by Stable Nitroxide Radicals. Chem Phys Lett 1992, 199, (6), 635-639.

184. Gijzeman, O. L.; Kaufman, F.; Porter, G., Quenching of aromatic triplet states in solution by nitric oxide and other free radicals J Chem Soc Farad T 2 1973, 69, (5), 727-737.

185. Richardson, C. F.; Schuster, D. I.; Wilson, S. R., Synthesis and Characterization of Water-Soluble Amino Fullerene Derivatives. Organic Letters 2000, 2, (8), 1011-1014.

186. Stoll, S.; Schweiger, A., Easyspin: Simulating CW ESR spectra. Bio Magn Res 2007, 27, 299-321.

187. Schweiger, A.; Jeschke, G., Principles of Pulse Electron Paramagnetic Resonance. Oxford University Press: New York, 2001. 


\section{References}

188. Forbes, M. D. E.; Jarocha, L. E.; Sim, S.; Tarasov, V. F., Time-Resolved Electron Paramagnetic Resonance Spectroscopy: History, Technique, and Application to Supramolecular and Macromolecular Chemistry. Adv Phys Org Chem 2013, 47, 1-83.

189. McLauchlan, K. A.; Stevens, D. G., Flash Photolysis Electron Spin Resonance. Accounts Chem Res 1988, 21, (2), 54-59.

190. Smaller, B.; Remko, J. R.; Avery, E. C., Electron Paramagnetic Resonance Studies of Transient Free Radicals Produced by Pulse Radiolysis. J Chem Phys 1968, 48, (11), 5174-5181.

191. Karatekin, E.; O'Shaughnessy, B.; Turro, N. J., Time-resolved EPR: A novel method for studying living chains. Macromolecules 1998, 31, (22), 7992-7995.

192. Clancy, C. M. R.; Tarasov, V. F.; Forbes, M. D. E., Time-resolved electron paramagnetic resonance studies in organic photochemistry. In Electron Paramagnetic Resonance: Volume 16, Atherton, N. M.; Davies, M. J.; Gilbert, B. C., Eds. The Royal Society of Chemistry: 1998; Vol. 16, pp 50-78.

193. Turro, N. J.; Kleinman, M. H.; Karatekin, E., Electron spin polarization and timeresolved electron paramagnetic resonance: Applications to the paradigms of molecular and supramolecular photochemistry. Angew Chem Int Edit 2000, 39, (24), 4436-4461.

194. Kim, S. T.; Sancar, A.; Essenmacher, C.; Babcock, G. T., Time-Resolved EPR Studies with DNA Photolyase: Excited-State FADH0 Abstracts an Electron from Trp-306 to Generate FADH-, the Catalytically Active Form of the Cofactor. P Natl Acad Sci USA 1993, 90, (17), 8023-8027.

195. Mahaney, J. E.; Froehlich, J. P.; Thomas, D. D., Conformational Transitions of the Sarcoplasmic Reticulum Ca-ATPase Studied by Time-Resolved EPR and QuenchedFlow Kinetics. Biochemistry 1995, 34, (14), 4864-4879.

196. Schlupmann, J.; Salikhov, K. M.; Plato, M.; Jaegermann, P.; Lendzian, F.; Mobius, K., Photoinduced Electron Transfer and Spin Dynamics in Mixed Porphyrin-Quinone Solutions Studied by Time-Resolved EPR. Appl Magn Reson 1991, 2, (1), 117-142.

197. Jockusch, S.; Zeika, O.; Jayaraj, N.; Ramamurthy, V.; Turro, N. J., Electron Spin Polarization Transfer from a Nitroxide Incarcerated within a Nanocapsule to a Nitroxide in the Bulk Aqueous Solution. J Phys Chem Lett 2010, 1, (18), 2628-2632.

198. Yamauchi, S.; Tanabe, M.; Ohba, Y.; Sugisaki, K.; Toyota, K.; Sato, K.; Takui, T.; Saltsman, I., Complete assignment of spin sublevels in the lowest excited triplet state of corrole compounds by time-resolved EPR spectroscopy. Chem Phys Lett 2012, 521, 6468 .

199. Krumkacheva, O.; Tanabe, M.; Yamauchi, S.; Fedin, M.; Marque, S. R. A.; Bagryanskaya, E., Time-Resolved and Pulse EPR Study of Triplet States of Alkylketones in beta-Cyclodextrin. Appl Magn Reson 2012, 42, (1), 29-40.

200. Closs, G. L.; Gautam, P.; Zhang, D.; Krusi, P. J.; Hill, S. A.; Wasserman, E., Steady-state and time-resolved direct detection EPR spectra of fullerene triplets in liquid solution and glassy matrices. Evidence for a dynamic Jahn-Teller effect in triplet C60. J Phys Chem 1992, 96, (13), 5228-5231.

201. Atkins, P. W.; Mclauchl.Ka; Simpson, A. F., High Resolution Transient ESR Spectra. Nature 1968, 219, (5157), 927-928. 


\section{References}

202. Atkins, P. W.; Buchanan, I. C.; Gurd, R. C.; Mclauchl.Ka; Simpson, A. F., Emission Electron Spin Resonance Spectra from Free Radicals in Solution. J Chem Soc Chem Comm 1970, (9), 513-514.

203. Livingston, R.; Zeldes, H., Paramagnetic Resonance Study of Liquids during Photolysis. VIII. An Emission Spectrum from Solutions of Tartaric Acid. The Journal of Chemical Physics 1970, 53, (4), 1406-1410.

204. Jockusch, S.; Porel, M.; Ramamurthy, V.; Turro, N. J., CIDEP from a Polarized Ketone Triplet State Incarcerated within a Nanocapsule to a Nitroxide in the Bulk Aqueous Solution. J Phys Chem Lett 2011, 2, (22), 2877-2880.

205. Kawai, A.; Mori, S.; Tsuji, K.; Shibuya, K., CIDEP Created by the Quenching of PhotoExcited Tryptophan at Protein Surface: A Challenge to CIDEP Probing of Protein Structural Changes. Appl Magn Reson 2010, 38, (2), 205-216.

206. Mclauchlan, K. A., Continuous-Wave Transient Electron Spin Resonance. In Modern Pulsed and Continuous-Wave Electron Spin Resonance, Kevan, L.; Bowman, M. K., Eds. John Wiley \& Sons, Inc.: New York, 1990; pp 285-363.

207. Bloch, F., Nuclear Induction. Phys. Rev. 1946, 70, (7-8), 460-474.

208. Mcconnell, H. M., Reaction Rates by Nuclear Magnetic Resonance. J Chem Phys 1958, 28, (3), 430-431.

209. Abergel, D.; Palmer, A. G., Approximate solutions of the Bloch-McConnell equations for two-site chemical exchange. Chemphyschem 2004, 5, (6), 787-793.

210. Schotland, J.; Leigh, J. S., Exact solutions of the Bloch equations with n-site chemical exchange. J Magn Reson 1983, 51, (1), 48-55.

211. Morris, G. A.; Chilvers, P. B., General Analytical Solutions of the Bloch Equations. $J$ Magn Reson Ser A 1994, 107, (2), 236-238.

212. Murase, K.; Tanki, N., Numerical solutions to the time-dependent Bloch equations revisited. Magn Reson Imaging 2011, 29, (1), 126-131.

213. Murase, K., Behavior of the magnetization in spin-locking magnetic resonance imaging using numerical solutions to the time-dependent Bloch equations. Phys Med Biol 2012, 57, (23), N481-N492.

214. Lingwood, M. D.; Han, S. I., Solution-State Dynamic Nuclear Polarization. Ann Rep NMR Spec 2011, 73, 83-126.

215. Krüger, G. J.; Müller-Warmuth, W.; Van Steenwinkel, R., Molecular Motion in Liquids and Solutions. II. Zeitschrift für Naturforschung A 1966, 21, 1224-1230.

216. Neudert, O.; Mattea, C.; Spiess, H. W.; Stapf, S.; Munnemann, K., A comparative study of 1H and 19F Overhauser DNP in fluorinated benzenes. Phys Chem Chem Phys 2013, 15, (47), 20717-20726.

217. Gafurov, M.; Denysenkov, V.; Prandolini, M. J.; Prisner, T. F., Temperature Dependence of the Proton Overhauser DNP Enhancements on Aqueous Solutions of Fremy's Salt Measured in a Magnetic Field of 9.2 T. Appl Magn Reson 2012, 43, (1-2), 119-128.

218. Neugebauer, P.; Krummenacker, J. G.; Denysenkov, V. P.; Parigi, G.; Luchinat, C.; Prisner, T. F., Liquid state DNP of water at 9.2 T: an experimental access to saturation. Phys Chem Chem Phys 2013, 15, (16), 6049-6056. 


\section{References}

219. Turke, M. T.; Bennati, M., Comparison of Overhauser DNP at 0.34 and $3.4 \mathrm{~T}$ with Fremy's Salt. Appl Magn Reson 2012, 43, (1-2), 129-138.

220. Eastman, M. P.; Kooser, R. G.; Das, M. R.; Freed, J. H., Studies of Heisenberg Spin Exchange in ESR Spectra. I. Linewidth and Saturation Effects. J Chem Phys 1969, 51, (6), 2690-2709.

221. Popp, C. A.; Hyde, J. S., Electron-Electron Double Resonance and Saturation-Recovery Studies of Nitroxide Electron and Nuclear Spin-Lattice Relaxation Times and Heisenberg Exchange Rates: Lateral Diffusion in Dimyristoyl Phosphatidylcholine. P Natl Acad Sci USA 1982, 79, (8), 2559-2563.

222. Currin, J. D., Theory of Exchange Relaxation of Hyperfine Structure in Electron Spin Resonance. Phys. Rev. 1962, 126, (6), 1995-2001.

223. Riplinger, C.; Kao, J. P. Y.; Rosen, G. M.; Kathirvelu, V.; Eaton, G. R.; Eaton, S. S.; Kutateladze, A.; Neese, F., Interaction of Radical Pairs Through-Bond and ThroughSpace: Scope and Limitations of the Point-Dipole Approximation in Electron Paramagnetic Resonance Spectroscopy. J Am Chem Soc 2009, 131, (29), 10092-10106.

224. Sezer, D., Computation of DNP coupling factors of a nitroxide radical in toluene: seamless combination of MD simulations and analytical calculations. Phys Chem Chem Phys 2013, 15, (2), 526-540.

225. Freed, J. H., Theory of Saturation and Double-Resonance Effects in ESR Spectra. J Chem Phys 1965, 43, (7), 2312-2332.

226. Freed, J. H., Theory of Saturation and Double Resonance Effects in Electron Spin Resonance Spectra. II. Exchange vs. Dipolar Mechanisms. J Phys Chem 1967, 71, (1), 38-51.

227. Enkin, N.; Liu, G. Q.; Tkach, I.; Bennati, M., High DNP efficiency of TEMPONE radicals in liquid toluene at low concentrations. Phys Chem Chem Phys 2014, 16, (19), 8795-8800.

228. Bales, B. L.; Peric, M., EPR line shifts and line shape changes due to spin exchange of nitroxide free radicals in liquids. $J$ Phys Chem B 1997, 101, (43), 8707-8716.

229. Brickmann, J; Kothe, G., ESR of the quartet states of randomly oriented molecules: Calculation of the line shape and detection of the zero-field splitting. J Chem Phys 1973, 59, (6), 2807-2814.

230. Bennati, M.; Grupp, A.; Mehring, M.; Dinse, K. P.; Fink, J., Pulsed EPR on the photoexcited triplet state of C60 fullerene. Chem Phys Lett 1992, 200, (5), 440-444. 


\section{List of Abbreviations}

$\begin{array}{ll}\text { BDPA } & \text { 1,3-bisdiphenylene-2-phenylallyl } \\ \text { bTbK } & \text { bis-TEMPO-bis-ketal } \\ \text { CE } & \text { cross effect } \\ \text { CIDEP } & \text { chemically induced dynamic electron polarization } \\ \text { CIDNP } & \text { chemically induced dynamic nuclear polarization } \\ \text { CT } & \text { charge transfer } \\ \text { CW } & \text { continuous wave } \\ \text { DEP } & \text { dynamic electron polarization } \\ \text { DNP } & \text { dynamic nuclear polarization } \\ \text { DSE } & \text { differential solid effect } \\ \text { DSSE } & \text { dressed-state solid effect } \\ \text { ELDOR } & \text { electron-electron double resonance } \\ \text { eNCP } & \text { electron-nuclear cross polarization } \\ \text { ENDOR } & \text { electron-nuclear double resonance } \\ \text { EPR } & \text { electron paramagnetic resonance } \\ \text { ESR } & \text { electron spin resonance } \\ \text { FID } & \text { free induction decay } \\ \text { FN } & \text { fullerene-nitroxide } \\ \text { FN-1a/2a/3a } & \text { fullerene-nitroxide mono/bis/trisadducts } \\ \text { FT } & \text { Fourier transformation } \\ \text { FWHM } & \text { full width at half maximum } \\ \text { HE } & \text { Heisenberg spin exchange } \\ \text { hf } & \text { hyperfine } \\ \text { HHCP } & \text { Hartmann-Hahn cross polarization } \\ \text { IC } & \text { internal conversion } \\ \text { ISC } & \text { intersystem crossing } \\ \text { ISE } & \text { integrated solid effect } \\ \text { LF/HF } & \text { low-field/high-field } \\ \text { MAS } & \text { magic-angle spinning } \\ \text { MD } & \text { molecular dynamics } \\ & \end{array}$


MG molten globule

MRI magnetic resonance imaging

MW microwave

NMR nuclear magnetic resonance

NMRD nuclear magnetic relaxation dispersion

NOVEL nuclear spin orientation via electron spin locking

OE Overhauser effect

PHIP para-hydrogen induced polarization

PR polarization recovery

RF radio frequency

RQM reversed quartet mechanism

SD spin diffusion

SE solid effect

TEMPO 2,2,6,6-tetramethylpiperidine-1-oxyl

TEMPOL 4-hydroxy-TEMPO

TEMPONE 4-oxo-TEMPO

TM thermal mixing

TOTAPOL 1-(TEMPO-4-oxy)-3-(TEMPO-4-amino)propan-2-ol

TrEPR transient electron paramagnetic resonance

ZFS zero field splitting 


\section{List of Figures}

1.1: Representation of the splitting of spin states $(|1\rangle=|-1 / 2\rangle$ and $|2\rangle=|+1 / 2\rangle)$ due to an external magnetic field.

1.2: Polarization of electron spins (e) and proton $\left({ }^{1} \mathrm{H}\right)$ as function of temperature at various external magnetic field strength values.

1.3: Right: Energy level diagrams and transition rates $\left(w_{S}\right.$ and $w_{I}$ are the probabilities of EPR and NMR transitions, respectively; $w_{0}$ and $w_{2}$ are the rates of zero and double quantum transitions, respectively); Left: Population distribution and Larmor frequencies of electron and nucleus $\left(\omega_{S}\right.$ and $\omega_{I}$, respectively).

1.4: The MW irradiation of the forbidden transitions equalizes the populations of correspondent spin states, which produces negative (left) or positive (right) DNP enhancement.

1.5: Illustration of electron (left) and nuclear (right) spin effective fields.

1.6: The coupling factor for different relaxation mechanisms (calculated for a correlation/diffusional time of $20 \mathrm{ps}$ )

1.7: DNP enhancement of water protons produced by nitroxide radical at different magnetic field. Black cycles: experimentally observed values. The maximum values (assuming $s=1, f=1$ ) predicted from MD calculation for TEMPO in water (red) and from force-free model (yellow) are given for a temperature range from 25 to $45^{\circ} \mathrm{C}$.

1.8: Simple schemes of the experimental approaches for DNP experiments.

1.9: The schematic of the first shuttle DNP spectrometer (left) and the modified shuttle DNP spectrometer with two-centers magnet (right).

1.10: The dependence of the coupling factor $\xi$ on the correlation time $\tau_{c}$. Right: the systems correspondent to the shown regimes of classification.

1.11: Illustrations of the introduction of radicals into reverse micelles. Left: covalently linked to the protein. Middle: dissolved in the aqueous core.

Right: attached to a carrier in the surfactant shell.......................................... 26

1.12: The most popular stable radicals/biradicals used in DNP experiments.

1.13: Possible strategy of spin polarization transfer from the photoexcited triplet state to nuclei.

2.1: Energy levels and pathways of the electron-proton coupled spin system.

2.2: Relaxation scheme for the $S=1 / 2$ and $I=1$ coupled spin system. The red arrow indicates a continuous MW irradiation at one of the hyperfine EPR transitions. The grey solid and dashed lines indicate electron $\left(w_{e}\right)$ and ${ }^{14} \mathrm{~N}$ 
nuclear $\left(w_{n}\right)$ spin relaxation pathways, respectively. The blue-cyan ones

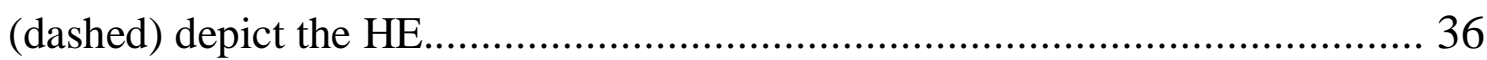

2.3: The nuclear relaxation rates as a function of the applied magnetic field (calculated for a correlation/diffusional time of $50 \mathrm{ps}$ ).

2.4: The field dependence of coupling factor for different relaxation mechanisms (calculated for a correlation/diffusional time of $20 \mathrm{ps}$ ).

2.5: The NMRD profile of the paramagnetic relaxation $\left(R_{I}\right.$ para $)$ for toluene protons with $1 \mathrm{mM}$ of TEMPONE at $298 \mathrm{~K}$. Orange dotted line: outer-sphere model only fitting ( $d=3.25 \AA$, $\left.D=2.46 \times 10^{-9} \mathrm{~m}^{2} / \mathrm{s}, \tau_{D}=43 \mathrm{ps}\right)$; Green solid line: inner-sphere and outer-sphere models together $(d=3.45 \AA$, $\left.D=2.42 \times 10^{-9} \mathrm{~m}^{2} / \mathrm{s}, \tau_{\text {dip }}=43 \mathrm{ps}\right)$

2.6: Scheme of dynamic electron polarization (DEP) concept............................. 48

2.7: Jablonski diagram of a molecule which can be excited to the triplet state.

2.8: The laser power dependence of the photoexcited triplet state for fullerene $C_{60}$ (laser beam section $\sigma=5 \mathrm{~mm}^{2}, k_{f} \sim 1 \mathrm{~ns}^{-1}, k_{i s c}=0.143 \mathrm{ps}^{-1}, \tau_{p}=5 \mu \mathrm{s}$ ). The quantum yield is around unity $\left(Y_{T l}=0.993\right)$.

2.9: Scheme of the superposition of a radical doublet $\left(\mathbf{D}_{\mathbf{0}}\right)$ with singlets and triplet states of a dye $\left(\mathbf{S}_{\mathbf{0}}, \mathbf{S}_{\mathbf{1}}\right.$ and $\mathbf{T}_{\mathbf{1}}$, respectively).

2.10: Jablonski diagram of the radical-dye system.

2.11: Kinetic scheme of processes in the radical-dye system according to the model of the reversed quartet mechanism. $k_{D, Q}$ and $k_{Q, D}$ are rates of transitions from $\mathbf{D}_{\mathbf{T} 1}$ to $\mathbf{Q}_{\mathbf{T} 1}$ and from $\mathbf{Q}_{\mathbf{T} 1}$ to $\mathbf{D}_{\mathbf{T} 1}$, respectively. $k_{D, D O}$ is the effective rate of $\mathbf{D}_{\mathrm{T} 1} \rightarrow \mathbf{D}_{\mathbf{S 0}}$ which includes several processes. $w_{i}(i=D O, D T, Q T)$ is transition probability between Zeeman sublevels of the corresponding states. $k_{Q, D O}$ is an ISC rate of $\mathbf{Q}_{\mathbf{T} 1} \rightarrow \mathbf{D}_{\mathbf{S 0} \text {. }}$

3.1: Fullerene-nitroxide derivatives investigated in DNP experiments (red cycle: link position).

3.2: Scheme of the synthesis of precursors FN (a) and $\mathbf{1}$ (b).

3.3: Scheme of the synthesis of fullerene-nitroxide derivatives by nucleophilic addition of the $\alpha$-bromomalonate carbanion derivated to FN (Bingel-type reaction).

3.4: UV-Vis absorption spectra of fullerene-nitroxide derivatives $(\sim 0.1 \mathrm{mM})$ in toluene at room temperature.

3.5: Normalized spectrum of an ELDOR experiment with TEMPOL radical in toluene solution. Here three signal reductions give the saturation factor values of the low field EPR line in the case of the MW irradiation of the low field (right), central (middle) and high field (left) EPR transitions. Insert: the scheme of the pulse sequence. 
3.6: Saturation recovery curves of FN-2a for pumping high field EPR transition. Insert: the scheme of the pulse sequence.

3.7: Scheme of the magnetization vector behaviour $\dot{M}(t) . \dot{B}_{0}$ is an external applied magnetic field; $B_{1}$ is a microwave field strength. Left: general case (off resonance); right: on resonance with initial condition $\dot{M}_{0}$

3.8: Pulse sequence of the pulsed EPR experiment with photoexcitation. Laser is used as an external source for the optical excitation to create excited transient (triplet) states or transient radicals as in the case of photolysis.

4.1: (a) CW-EPR spectra of TEMPONE- $\mathrm{D}_{16-}{ }^{15} \mathrm{~N}$ in toluene at different concentrations at $\mathrm{MW}$ power $2 \mathrm{~mW}$. Experimental parameters: $1 \mathrm{G}$ modulation amplitude, $100 \mathrm{kHz}$ modulation frequency, $5.12 \mathrm{~ms}$ conversion time, 1024 points. (b) Peak-to-trough intensity power dependence of the low field EPR line (the derivative of Lorentzian line) for TEMPONE-D $16^{-}{ }^{15} \mathrm{~N}$ in toluene at different concentrations and fitting curves. 70

4.2: (a) ${ }^{1} \mathrm{H}$ NMR spectra of toluene doped with $5 \mathrm{mM}$ TEMPONE- $\mathrm{D}_{16^{-}}{ }^{15} \mathrm{~N}$ at $14 \mathrm{MHz}$; (b) concentration dependence of DNP enhancements (from maximum of $\varepsilon_{F I D}$ ) of TEMPONE-D ${ }_{16}{ }^{15} \mathrm{~N}$ in toluene and in water.

4.3: Concentration dependence of the leakage factor $f$ in toluene and water doped with TEMPONE- $\mathrm{D}_{16^{-}}{ }^{15} \mathrm{~N}$ at $0.35 \mathrm{~T}$.

4.4: (a) EPR-FID intensity of the high-field hyperfine line of TEMPONE- $\mathrm{D}_{16}{ }^{15} \mathrm{~N}$ as a function of the frequency of the saturating (pumping) frequency. (b) Concentration dependence of $s_{2, \max }$, the solid curves are the plots of the equation (4.1) for toluene and water systems with the kinetic parameters obtained from saturation recovery experiments.

4.5: Polarization recovery curves and fits of the irradiated EPR line (blue) and the hyperfine coupled line (red) at $1.6 \mathrm{mM}$ TEMPONE- $\mathrm{D}_{16}{ }^{15} \mathrm{~N}$ in toluene.

5.1: (a) Normalized CW-EPR spectra of fullerene-nitroxide derivatives in toluene $(1.5 \mathrm{mM})$. Black solid lines are fits according to the fast motion regime. At $2 \mathrm{~mW}$ of the MW power, $1 \mathrm{G}$ and $100 \mathrm{kHz}$ of modulation amplitude and frequency, respectively, room temperature. (b) MW power dependence of the peak-to-peak intensity of the central nitroxide hyperfine line. Solid curves are fitting.

5.2: (a) Boltzmann (blue) and DNP (red) $14 \mathrm{MHz}{ }^{1} \mathrm{H}-\mathrm{NMR}$ spectra recorded in toluene solution of FN-2a. (b) DNP enhancements for both ring and methyl protons of toluene with different polarizers.

5.3: Normalized FID intensities of TL (a), FN (b), FN-1a (c), FN-2a (d) and FN-3a (e), respectively, as a function of the pumping frequency. 
5.4: Polarization Recovery (PR) curves and fits (solid lines) of the TL (a), FN (b), FN-1a (c), FN-2a (d), FN-3a (e), respectively, for the case of pumping on the high field line $\left(t_{\text {pump }}=0.1 \mu \mathrm{s}\right)$. The polarizer concentration is $\sim 1.5 \mathrm{mM}$.

5.5: (a) ${ }^{14} \mathrm{~N}$ nuclear relaxation $2 w_{n}\left(1 / T_{l n}\right)$ and $\mathrm{HE}$ rate $K_{x} N$ (with polarizer concentration $N \sim 1.5 \mathrm{mM}$ ). $2 w_{n}$ values for $\mathbf{T L}$ and $\mathbf{F N}$ and $K_{x} N$ value for FN-3a turned out to be negligible in the fits and are given as upper limit. The errors of up to $20 \%$ were estimated from the experiments with various polarizer concentrations. (b) Simulation of the ${ }^{14} \mathrm{~N}$ nuclear relaxation according to the electron-nuclear dipolar mechanism (5.2).

5.6: The polarizer concentration dependence of the effective saturation factor when the central EPR transition is irradiated. The experimental values (red circles and black squares) are from the ELDOR experiments. The solid lines are theoretically simulated curves using the rate parameters obtained from fitting polarization recovery curves

6.1: TrEPR spectra of photoexcited $\mathbf{F N}$ (red) and $\mathrm{C}_{60}$ triplet state (light purple). $g_{R} \approx 2.0077, A_{R} \approx 15.25 \mathrm{G}$ and $g_{T} \approx 2.0029$.

6.2: TrEPR spectra of FN (a), FN-1a (b), FN-2a (c), FN-3a (d) recorded at the described time delay after a laser flash (insert).

6.3: Time evolution of EPR signals of $1^{\text {st }}$ (color) and $3^{\text {rd }}$ (black) lines for $\mathbf{F N}$ (a), FN-1a (b), FN-2a (c) and FN-3a (d).

6.4: The normalized low field line FID intensity $\left(I_{F I D} / I_{F I D, B o l t z m a n n}\right)$ of $\mathbf{F N}$ in toluene as a function of the delay time for photoexcited (red) and Boltzmann (white) states. Insert: the FID traces measured at the delay time marked by a blue arrow. Experimental details: sample concentration $\sim 0.8 \mathrm{mM}$, volume $\sim 20 \mu \mathrm{L}, \mathrm{MW}$ “ $\pi / 2$ ” length $20 \mathrm{~ns}$.

6.5: Kinetic trace of the photoexcited triplet state of $\mathrm{C}_{60}$ in toluene solution $(1.5 \mathrm{mM}, 20 \mu \mathrm{L})$ by pulsed time-resolved EPR. Insert: FID decay of the peak of ${ }^{3} \mathrm{C}_{60}$ signal (black arrow). 


\section{List of Tables}

3.1: Ratio of reactants in the synthesis of fullerene-nitroxide derivatives

4.1: Summary of DNP parameters for toluene doped with TEMPONE-D $16^{-}{ }^{15} \mathrm{~N} \ldots .80$

5.1: Comparison of the saturation factor values experimentally determined from ELDOR spectra $\left(s^{e x p}\right)$ and theoretically calculated using the analytical equations and the rate parameters $\left(2 w_{e}, 2 w_{n}, K_{x} N\right)$ obtained from the fitting of polarization recovery curves $\left(s^{\text {theory }}\right)$.

5.2: Summary of DNP parameters, relaxation times and Heisenberg exchange rate constants for toluene solutions of $\mathbf{T L}$ and $\mathbf{F N}$ derivatives. 96 


\section{Appendix}

Mathematica, Wolfram Research, Inc. Program for estimating the relaxation rate between hyperfine lines

Style["B1 irradiation", Red, 17]

SatRec3oB1[t_, s_] := (-A1^2*Exp[-t/ $\mathrm{ATau}] 1]-\mathrm{B} 1^{\wedge} 2^{*} \operatorname{Exp}[-\mathrm{t} / \wedge[\mathrm{Tau}] 2]-\mathrm{C} 1 \wedge 2 * \operatorname{Exp}[-$ $\mathrm{t} \wedge[\mathrm{Tau}] 3]+\mathrm{D} 1)^{*}(1-\mathrm{s}) *(2-\mathrm{s})^{*} 0.5+\left(-\mathrm{A} 2^{\wedge} 2^{*} \operatorname{Exp}[-\mathrm{t} /[\mathrm{Tau}] 1]+\mathrm{C} 2^{\wedge} 2^{*} \operatorname{Exp}[-\mathrm{t} / \wedge[\mathrm{Tau}] 3]\right.$ $+\mathrm{D} 2)^{*} \mathrm{~s}^{*}(2-\mathrm{s})+\left(-\mathrm{A} 3^{\wedge} 2^{*} \operatorname{Exp}[-\mathrm{t} \wedge[\mathrm{Tau}] 1]+\mathrm{B} 3^{\wedge} 2^{*} \operatorname{Exp}[-\mathrm{t} \wedge[\mathrm{Tau}] 2]-\mathrm{C} 3^{\wedge} 2^{*} \operatorname{Exp}[-\right.$ $\mathrm{t} \wedge[\mathrm{Tau}] 3]+\mathrm{D} 3) * \mathrm{~s} *(1-\mathrm{s}) *(-0.5)$;

TLint1p6sRecNIFoB1 = NonlinearModelFit[TLint1p6sRecforFit[[1]], \{SatRec3oB1[t, $\mathrm{s}]\},\{\{\backslash[\mathrm{Tau}] 1,380\},\{\backslash[\mathrm{Tau}] 2,110\},\{\backslash[\mathrm{Tau}] 3,90\}, \mathrm{A} 1, \mathrm{~B} 1,\{\mathrm{C} 1,-0.1\}, \mathrm{A} 2,\{\mathrm{C} 2$, $0.1\}, A 3, B 3,\{C 3,-0.1\},\{D 1,1\},\{D 2,1\},\{D 3,1\}\},\{t, s\}$, Method $\rightarrow$ "LevenbergMarquardt"]

FTint1p6sRecNIFoB1 = NonlinearModelFit[FTint1p6sRecforFit[[1]], $\{$ SatRec3oB1[t, $\mathrm{s}]\},\{\{\backslash[\mathrm{Tau}] 1,380\},\{\backslash[\mathrm{Tau}] 2,110\},\{\backslash \mathrm{Tau}] 3,90\}, \mathrm{A} 1, \mathrm{~B} 1,\{\mathrm{C} 1,-0.1\}, \mathrm{A} 2,\{\mathrm{C} 2$, $0.1\}, A 3, B 3,\{C 3,-0.1\},\{D 1,1\},\{D 2,1\},\{D 3,1\}\},\{t, s\}$, Method $->$

"LevenbergMarquardt"]

FT1aint1p6sRecNIFoB1 = NonlinearModelFit[FT1aint1p6sRecforFit[[1]], $\{$ SatRec3oB1[t, s] $\},\{\{\backslash$ Tau $] 1,2500\},\{\backslash[\mathrm{Tau}] 2,200\},\{\backslash[\mathrm{Tau}] 3,100\}, \mathrm{A} 1, \mathrm{~B} 1,\{\mathrm{C} 1,-$ $0.1\}, A 2,\{C 2,0.1\}, A 3, B 3,\{C 3,-0.1\},\{\mathrm{D} 1,1\},\{\mathrm{D} 2,1\},\{\mathrm{D} 3,1\}\},\{\mathrm{t}, \mathrm{s}\}$, Method -> "LevenbergMarquardt"]

FT2aint1p6sRecNIFoB1 = NonlinearModelFit[FT2aint1p6sRecforFit[[1]], $\{$ SatRec3oB1[t, s] $\},\{\{\backslash[\mathrm{Tau}] 1,3700\},\{\backslash[\mathrm{Tau}] 2,200\},\{\backslash[\mathrm{Tau}] 3,100\}, \mathrm{A} 1, \mathrm{~B} 1,\{\mathrm{C} 1,-$ $0.1\}, \mathrm{A} 2,\{\mathrm{C} 2,0.1\}, \mathrm{A} 3, \mathrm{~B} 3,\{\mathrm{C} 3,-0.1\},\{\mathrm{D} 1,1\},\{\mathrm{D} 2,1\},\{\mathrm{D} 3,1\}\},\{\mathrm{t}, \mathrm{s}\}$, Method -> "LevenbergMarquardt"]

FT3aint1p6sRecNlFoB1 = NonlinearModelFit[FT3aint1p6sRecforFit[[1]], $\{$ SatRec3oB1[t, s] $\},\{\{\backslash[\mathrm{Tau}] 1,4000\},\{\backslash[\mathrm{Tau}] 2,200\},\{\backslash[\mathrm{Tau}] 3,90\}, \mathrm{A} 1, \mathrm{~B} 1,\{\mathrm{C} 1,-$ $0.1\}, \mathrm{A} 2,\{\mathrm{C} 2,0.1\}, \mathrm{A} 3, \mathrm{~B} 3,\{\mathrm{C} 3,-0.1\},\{\mathrm{D} 1,1\},\{\mathrm{D} 2,1\},\{\mathrm{D} 3,1\}\},\{\mathrm{t}, \mathrm{s}\}$, Method -> "LevenbergMarquardt"]

Style["B2 irradiation", Red, 17] 
SatRec3oB2[t_, s_] := (-A1^2* $\operatorname{Exp}[-\mathrm{t} \wedge[\mathrm{Tau}] 1]+\mathrm{C} 1 \wedge 2 * \operatorname{Exp}[-\mathrm{t} \wedge[\mathrm{Tau}] 3]+\mathrm{D} 1)^{*}(1-$ $\mathrm{s}) *(2-\mathrm{s}) * 0.5+\left(-\mathrm{A} 2 \wedge 2^{*} \operatorname{Exp}[-\mathrm{t} \wedge[\mathrm{Tau}] 1]-\mathrm{C} 2^{\wedge} 2 * \operatorname{Exp}[-\mathrm{t} \wedge[\mathrm{Tau}] 3]+\mathrm{D} 2\right)^{*} \mathrm{~s} *(2-\mathrm{s})+(-$ $\left.\mathrm{A} 3^{\wedge} 2 * \operatorname{Exp}[-\mathrm{t} / \wedge[\mathrm{Tau}] 1]+\mathrm{C} 3^{\wedge} 2 * \operatorname{Exp}[-\mathrm{t} /[\mathrm{Tau}] 3]+\mathrm{D} 3\right) * \mathrm{~s} *(1-\mathrm{s}) *(-0.5)$

TLint1p6sRecNlFoB2 = NonlinearModelFit[TLint1p6sRecforFit[[2]], $\{$ SatRec3oB2[t, $\mathrm{s}]\},\{\{\backslash \mathrm{Tau}] 1,380\},\{\backslash[\mathrm{Tau}] 3,100\}, \mathrm{A} 1,\{\mathrm{C} 1,-0.1\}, \mathrm{A} 2,\{\mathrm{C} 2,0.1\}, \mathrm{A} 3,\{\mathrm{C} 3,-0.1\}$, $\{\mathrm{D} 1,1\},\{\mathrm{D} 2,1\},\{\mathrm{D} 3,1\}\},\{\mathrm{t}, \mathrm{s}\}$, Method -> "LevenbergMarquardt"]

FTint1p6sRecN1FoB2 = NonlinearModelFit[FTint1p6sRecforFit[[2]], $\{$ SatRec3oB2[t, $\mathrm{s}]\},\{\{\backslash \mathrm{Tau}] 1,380\},\{\backslash[\mathrm{Tau}] 3,100\}, \mathrm{A} 1,\{\mathrm{C} 1,-0.1\}, \mathrm{A} 2,\{\mathrm{C} 2,0.1\}, \mathrm{A} 3,\{\mathrm{C} 3,-0.1\}$, $\{\mathrm{D} 1,1\},\{\mathrm{D} 2,1\},\{\mathrm{D} 3,1\}\},\{\mathrm{t}, \mathrm{s}\}$, Method -> "LevenbergMarquardt"]

FT1aint1p6sRecNlFoB2 = NonlinearModelFit[FT1aint1p6sRecforFit[[2]], $\{$ SatRec3oB2[t, s] $\},\{\{\backslash[\mathrm{Tau}] 1,380\},\{\backslash[\mathrm{Tau}] 3,100\}, \mathrm{A} 1,\{\mathrm{C} 1,-0.1\}, \mathrm{A} 2,\{\mathrm{C} 2,0.1\}$, A3, $\{\mathrm{C} 3,-0.1\},\{\mathrm{D} 1,1\},\{\mathrm{D} 2,1\},\{\mathrm{D} 3,1\}\},\{\mathrm{t}, \mathrm{s}\}$, Method $->$ "LevenbergMarquardt"]

FT2aint1p6sRecNlFoB2 = NonlinearModelFit[FT2aint1p6sRecforFit[[2]], $\{$ SatRec3oB2[t, s] $\},\{\{\backslash[\mathrm{Tau}] 1,3800\},\{\backslash[\mathrm{Tau}] 3,100\}, \mathrm{A} 1,\{\mathrm{C} 1,-0.1\}, \mathrm{A} 2,\{\mathrm{C} 2,0.1\}$, A3, $\{C 3,-0.1\},\{D 1,1\},\{D 2,1\},\{D 3,1\}\},\{t, s\}$, Method -> "LevenbergMarquardt"]

FT3aint1p6sRecNlFoB2 = NonlinearModelFit[FT3aint1p6sRecforFit[[2]],

$\{$ SatRec3oB2[t, s] $\},\{\{\backslash$ Tau $] 1,3800\},\{\backslash[$ Tau $] 3,100\}, A 1,\{\mathrm{C} 1,-0.1\}, A 2,\{\mathrm{C} 2,0.1\}$, A3, $\{C 3,-0.1\},\{D 1,1\},\{D 2,1\},\{D 3,1\}\},\{t, s\}$, Method $->$ "LevenbergMarquardt"]

Style["B3 irradiation", Red, 17]

SatRec3oB3[t_, s_] := (-A1^2*Exp[-t/ $[\mathrm{Tau}] 1]+\mathrm{B} 1 \wedge 2 * \operatorname{Exp}[-\mathrm{t} \wedge[\mathrm{Tau}] 2]-\mathrm{C} 1 \wedge 2 * \operatorname{Exp}[-$ $\mathrm{t} \wedge[\mathrm{Tau}] 3]+\mathrm{D} 1)^{*}(1-\mathrm{s}) *(2-\mathrm{s}) * 0.5+\left(-\mathrm{A} 2^{\wedge} 2 * \operatorname{Exp}[-\mathrm{t} / \wedge[\mathrm{Tau}] 1]+\mathrm{C} 2 \wedge 2 * \operatorname{Exp}[-\mathrm{t} / \mathrm{T}\right.$ Tau $\left.] 3\right]$ $+\mathrm{D} 2)^{*} \mathrm{~s} *(2-\mathrm{s})+\left(-\mathrm{A} 3^{\wedge} 2^{*} \operatorname{Exp}[-\mathrm{t} \wedge[\mathrm{Tau}] 1]-\mathrm{B} 3^{\wedge} 2^{*} \operatorname{Exp}[-\mathrm{t} \wedge[\mathrm{Tau}] 2]-\mathrm{C} 3^{\wedge} 2^{*} \operatorname{Exp}[-\right.$ $\mathrm{t} \wedge[\mathrm{Tau}] 3]+\mathrm{D} 3) * \mathrm{~s} *(1-\mathrm{s}) *(-0.5)$;

TLint1p6sRecNlFoB3 = NonlinearModelFit[TLint1p6sRecforFit[[3]], $\{$ SatRec3oB3[t, $\mathrm{s}]\},\{\{\backslash[\mathrm{Tau}] 1,380\},\{\backslash \mathrm{Tau}] 2,200\},\{\backslash[\mathrm{Tau}] 3,90\}, \mathrm{A} 1, \mathrm{~B} 1,\{\mathrm{C} 1,0.1\}, \mathrm{A} 2,\{\mathrm{C} 2,0.1\}$, A3, B3, $\{\mathrm{C} 3,0.1\},\{\mathrm{D} 1,1\},\{\mathrm{D} 2,1\},\{\mathrm{D} 3,1\}\},\{\mathrm{t}, \mathrm{s}\}$, Method $->$ "LevenbergMarquardt"]

FTint1p6sRecNlFoB3 = NonlinearModelFit[FTint1p6sRecforFit[[3]], $\{$ SatRec3oB3[t, $\mathrm{s}]\},\{\{\backslash[\mathrm{Tau}] 1,1100\},\{\backslash[\mathrm{Tau}] 2,180\},\{\backslash[\mathrm{Tau}] 3,120\}, \mathrm{A} 1, \mathrm{~B} 1,\{\mathrm{C} 1,0.1\}, \mathrm{A} 2,\{\mathrm{C} 2$, $0.1\}, A 3, B 3,\{C 3,0.1\},\{D 1,1\},\{D 2,1\},\{D 3,1\}\},\{t, s\}$, Method $->$ "LevenbergMarquardt"]

FT1aint1p6sRecNlFoB3 = NonlinearModelFit[FT1aint1p6sRecforFit[[3]], $\{$ SatRec3oB3[t, s] $\},\{\{\backslash[$ Tau $] 1,380\},\{\backslash[$ Tau $] 2,200\},\{\backslash[$ Tau $] 3,120\}, A 1, B 1,\{\mathrm{C} 1$, 
$0.1\}, A 2,\{C 2,0.1\}, A 3, B 3,\{C 3,0.1\},\{\mathrm{D} 1,1\},\{\mathrm{D} 2,1\},\{\mathrm{D} 3,1\}\},\{\mathrm{t}, \mathrm{s}\}$, Method -> "LevenbergMarquardt"]

FT2aint1p6sRecNlFoB3 = NonlinearModelFit[FT2aint1p6sRecforFit[[3]], $\{$ SatRec3oB3[t, s] $\},\{\{\backslash[\mathrm{Tau}] 1,4000\},\{\backslash[\mathrm{Tau}] 2,200\},\{\backslash[\mathrm{Tau}] 3,100\}, \mathrm{A} 1, \mathrm{~B} 1,\{\mathrm{C} 1,-$ $0.1\}, A 2,\{\mathrm{C} 2,0.1\}, \mathrm{A} 3, \mathrm{~B} 3,\{\mathrm{C} 3,-0.1\},\{\mathrm{D} 1,1\},\{\mathrm{D} 2,1\},\{\mathrm{D} 3,1\}\},\{\mathrm{t}, \mathrm{s}\}$, Method -> "LevenbergMarquardt"]

FT3aint1p6sRecNlFoB3 = NonlinearModelFit[FT3aint1p6sRecforFit[[3]], $\{$ SatRec3oB3[t, s]\}, $\{\{\backslash[\mathrm{Tau}] 1,3800\},\{\backslash[\mathrm{Tau}] 2,200\},\{\backslash[\mathrm{Tau}] 3,120\}, \mathrm{A} 1, \mathrm{~B} 1,\{\mathrm{C} 1$, $0.1\}, A 2,\{C 2,0.1\}, A 3, B 3,\{C 3,0.1\},\{\mathrm{D} 1,1\},\{\mathrm{D} 2,1\},\{\mathrm{D} 3,1\}\},\{\mathrm{t}, \mathrm{s}\}$, Method -> "LevenbergMarquardt"] 


\section{Curriculum Vitae}

\section{Personal data}
Name:
Nikolay A. Enkin
Date of birth:
10 March 1987
Place of birth:
Moscow, Russia

\section{Education}

2011-2015 PhD student

Max Planck Institute for Biophysical Chemistry, Göttingen, Germany

Thesis title: "Fullerene-Nitroxide Derivatives as Potential Polarizers for Dynamic Nuclear Polarization (DNP) in Liquid State"

Supervisor: Prof. Dr. Marina Bennati

2008-2010 MSc in Applied Mathematics and Physics

Moscow Institute of Physics and Technology (State University), Moscow, Russia

Thesis title: "Homogenization of the Maxwell equations for layered media"

Supervisor: Dr. Aleksandr M. Merzlikin, Prof. Dr. Aleksey P. Vinogradov

2004-2008 BSc in Applied Mathematics and Physics

Moscow Institute of Physics and Technology (State University), Moscow, Russia

Thesis title: "Calculations of Navier-Stokes equations including diffusion, thermal conductivity and coefficients of turbulent transport" Supervisor: Prof. Dr. Mikhail F. Ivanov 


\section{Fellowships}

2010 Participant of Scholarship Programme of Foundation “Dynasty”.

\section{Publications}

N. A. Enkin, A. M. Merzlikin, Non-Fresnel's refraction laws of composite materials. Proceedings of 6th International Conference of Young Scientists and Professionals “Optics-2009”. St. Petersburg: Saint-Petersburg State University of Information Technology, Mechanics and Optics, 2009, 165-167.

N. A. Enkin, A. M. Merzlikin, A. P. Vinogradov, Refraction law deviation of composite materials from Fresnel's law. Journal of Communications Technology and Electronics, 2010, Vol.55, N.5, 565-571.

N. Enkin, G. Liu, I. Tkach and M. Bennati, High DNP efficiency of TEMPONE radicals in liquid toluene at low concentrations, Phys Chem Chem Phys, 2014, 16, 8795-8800.

N. Enkin, G. Liu, M. d. C. Gimenez-Lopez, K. Porfyrakis, I. Tkach and M. Bennati, A high saturation factor in Overhauser DNP with nitroxide derivatives: the role of $14 \mathrm{~N}$ nuclear spin relaxation, Phys Chem Chem Phys, 2015, 17, 11144-11149

\section{Conference/Seminar Contributions}

2012 COST Summer School on Spin-Hyperpolarization, Leiden, Netherlands.

2014 Poster, $11^{\text {th }}$ EUROMAR conference, Zürich, Switzerland. 